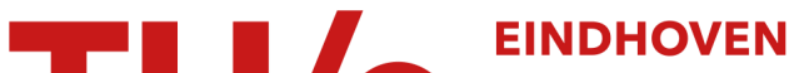 \\ UNIVERSITY OF \\ TECHNOLOGY
}

\section{Metastability and nucleation for conservative dynamics}

\section{Citation for published version (APA):}

Hollander, den, W. T. F., Olivieri, E., \& Scoppola, E. (2000). Metastability and nucleation for conservative dynamics. Journal of Mathematical Physics, 41(3), 1424-1498. https://doi.org/10.1063/1.533193

DOI:

$10.1063 / 1.533193$

Document status and date:

Published: 01/01/2000

\section{Document Version:}

Publisher's PDF, also known as Version of Record (includes final page, issue and volume numbers)

\section{Please check the document version of this publication:}

- A submitted manuscript is the version of the article upon submission and before peer-review. There can be important differences between the submitted version and the official published version of record. People interested in the research are advised to contact the author for the final version of the publication, or visit the $\mathrm{DOI}$ to the publisher's website.

- The final author version and the galley proof are versions of the publication after peer review.

- The final published version features the final layout of the paper including the volume, issue and page numbers.

Link to publication

\section{General rights}

Copyright and moral rights for the publications made accessible in the public portal are retained by the authors and/or other copyright owners and it is a condition of accessing publications that users recognise and abide by the legal requirements associated with these rights.

- Users may download and print one copy of any publication from the public portal for the purpose of private study or research.

- You may not further distribute the material or use it for any profit-making activity or commercial gain

- You may freely distribute the URL identifying the publication in the public portal.

If the publication is distributed under the terms of Article 25fa of the Dutch Copyright Act, indicated by the "Taverne" license above, please follow below link for the End User Agreement:

www.tue.nl/taverne

Take down policy

If you believe that this document breaches copyright please contact us at:

openaccess@tue.nl

providing details and we will investigate your claim. 


\section{Metastability and nucleation for conservative dynamics}

F. den Hollander

Mathematisch Instituut, Universiteit Nijmegen, Toernooiveld 1, 6525 ED Nijmegen, The Netherlands

E. Olivieri

Dipartimento di Matematica, Università di Roma Tor Vergata, Via della Ricerca Scientifica, 00133 Rome, Italy

E. Scoppola

Dipartimento di Matematica, Università di Roma Tre, Largo S. Leonardo Murialdo 1, 00146 Rome, Italy

(Received 18 November 1999; accepted for publication 7 December 1999)

In this paper we study metastability and nucleation for a local version of the two-dimensional lattice gas with Kawasaki dynamics at low temperature and low density. Let $\beta>0$ be the inverse temperature and let $\bar{\Lambda} \subset \Lambda_{\beta} \subset \mathbb{Z}^{2}$ be two finite boxes. Particles perform independent random walks on $\Lambda_{\beta} \backslash \bar{\Lambda}$ and inside $\bar{\Lambda}$ feel exclusion as well as a binding energy $U>0$ with particles at neighboring sites, i.e., inside $\bar{\Lambda}$ the dynamics follows a Metropolis algorithm with an attractive lattice gas Hamiltonian. The initial configuration is chosen such that $\bar{\Lambda}$ is empty, while a total of $\rho\left|\Lambda_{\beta}\right|$ particles is distributed randomly over $\Lambda_{\beta} \backslash \bar{\Lambda}$ with no exclusion. That is to say, initially the system is in equilibrium with particle density $\rho$ conditioned on $\bar{\Lambda}$ being empty. For large $\beta$, the system in equilibrium has $\bar{\Lambda}$ fully occupied because of the binding energy. We consider the case where $\rho=e^{-\Delta \beta}$ for some $\Delta$ $\in(U, 2 U)$ and investigate how the transition from empty to full takes place under the dynamics. In particular, we identify the size and shape of the critical droplet and the time of its creation in the limit as $\beta \rightarrow \infty$ for fixed $\Lambda$ and $\lim _{\beta \rightarrow \infty}(1 / \beta) \log \left|\Lambda_{\beta}\right|=\infty$. In addition, we obtain some information on the typical trajectory of the system prior to the creation of the critical droplet. The choice $\Delta$ $\in(U, 2 U)$ corresponds to the situation where the critical droplet has side length $l_{c} \in(1, \infty)$, i.e., the system is metastable. The side length of $\bar{\Lambda}$ must be much larger than $l_{c}$ and independent of $\beta$, but is otherwise arbitrary. Because particles are conserved under Kawasaki dynamics, the analysis of metastability and nucleation is more difficult than for Ising spins under Glauber dynamics. The key point is to show that at low density the gas in $\Lambda_{\beta} \backslash \bar{\Lambda}$ can be treated as a reservoir that creates particles with rate $\rho$ at sites on the interior boundary of $\bar{\Lambda}$ and annihilates particles with rate 1 at sites on the exterior boundary of $\bar{\Lambda}$. Once this approximation has been achieved, the problem reduces to understanding the local metastable behavior inside $\bar{\Lambda}$ in the presence of a nonconservative boundary. The dynamics inside $\bar{\Lambda}$ is still conservative and this difficulty has to be handled via local geometric arguments. Here it turns out that the Kawasaki dynamics has its own peculiarities. For instance, rectangular droplets tend to become square through a movement of particles along the border of the droplet. This is different from the behavior under the Glauber dynamics, where subcritical rectangular droplets are attracted by the maximal square contained in the interior, while supercritical rectangular droplets tend to grow uniformly in all directions (at least for not too long a time) without being attracted by a square. (ㄷ 2000 American Institute of Physics.

[S0022-2488(00)01503-6] 


\section{INTRODUCTION AND MAIN RESULTS}

In this paper we study metastability for conservative (C) dynamics. In particular, we study the transition to the liquid phase of a supersaturated vapor described by a local version of the twodimensional lattice gas with Kawasaki dynamics at low temperature and low density.

Metastability is a relevant phenomenon for thermodynamic systems close to a first-order phase transition. Suppose the system is in a pure equilibrium phase, corresponding to a point in the phase diagram close to a first-order phase transition curve. Suppose we change the thermodynamic parameters to values associated with a different equilibrium phase, corresponding to a point on the opposite side of the curve. Then, in certain experimental situations, instead of undergoing a phase transition the system can remain in the old equilibrium, far from the new equilibrium, for a long time. This unstable old equilibrium, called metastable state, persists until an external perturbation or a spontaneous fluctuation leads the system to the stable new equilibrium.

Examples of metastable states are supersaturated vapor and solutions, supercooled liquids, and ferromagnets with a magnetization opposite to the magnetic field.

In Sec. 1.1 we recall some of the main features of metastability by describing some wellknown results obtained for a nonconservative (NC) dynamics, namely Ising spins with Glauber dynamics. In Sec. 1.2 we introduce a conservative model, namely the lattice gas with Kawasaki dynamics, and discuss the main differences between C and NC. In Sec. 1.3 we propose a simplified model, where the interaction and the exclusion only act in a finite box, and formulate our main theorem establishing metastable behavior for this model. In Sec. 1.4 we give an outline of the key ideas needed to prove this theorem, which are further developed in the remainder of the paper. In Sec. 1.5 we collect some additional notation that is used throughout the paper.

\subsection{The non-conservative case}

1.1.1. Grand-canonical ensemble. Let $\Lambda \subset Z^{2}$ be a large finite box centered at the origin. With each site $x \in \Lambda$ we associate a spin variable $\sigma(x)$, assuming the values +1 or -1 . With each configuration $\sigma \in \mathcal{X}=\{-1,+1\}^{\Lambda}$ we associate an energy

$$
H(\sigma)=-\frac{J}{2} \sum_{(x, y) \in \Lambda^{*}} \sigma(x) \sigma(y)-\frac{h}{2} \sum_{x \in \Lambda} \sigma(x),
$$

where $\Lambda^{*}$ is the set of bonds between nearest-neighbor sites in $\Lambda, J>0$ is the pair interaction, $h>0$ is the magnetic field, and we assume periodic boundary conditions on $\Lambda$. The grandcanonical Gibbs measure associated with the Hamiltonian $H$, describing the equilibrium properties of the system, is given by

$$
\mu(\sigma)=\frac{e^{-\beta H(\sigma)}}{Z} \quad(\sigma \in \mathcal{X})
$$

where $Z$ is the partition function

$$
Z=\sum_{\sigma \in \mathcal{X}} e^{-\beta H(\sigma)}
$$

and $\beta>0$ is the inverse temperature. The qualification "grand-canonical" is used here because $h$ plays the role of a chemical potential and the total magnetization $\Sigma_{x \in \Lambda} \sigma(x)$ is not constant under $\mu$.

It is well known that for every $J, h, \beta>0$ in the thermodynamic limit $\Lambda \rightarrow Z^{2}$ a unique Gibbs state with a positive magnetization exists (see e.g., Ruelle ${ }^{1}$ and Sinai $^{2}$ ). We will be interested in the regime where

$$
\Lambda \text { is large but finite, } h \in(0,2 J), \quad \beta \rightarrow \infty \text {. }
$$




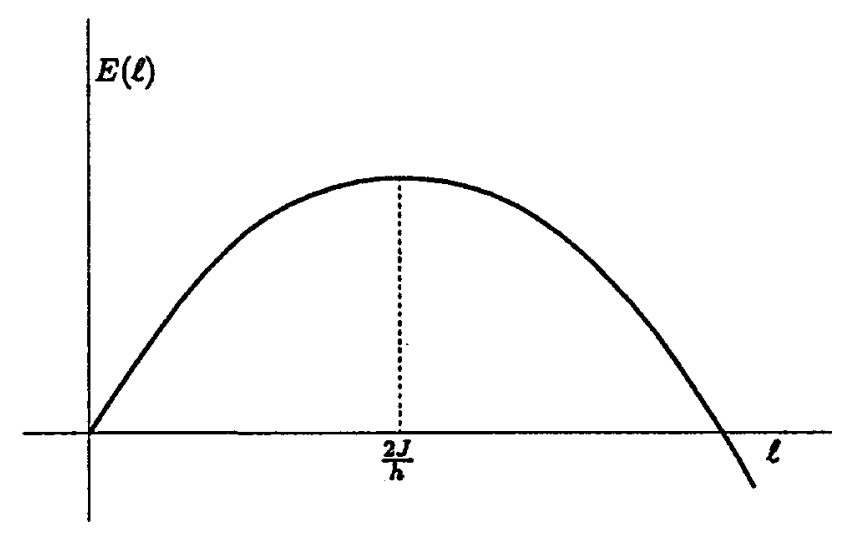

FIG. 1. The energy of an $l \times l$ droplet (NC).

$$
\begin{aligned}
& \ominus=\text { the configuration with } \sigma(x)=+1 \text { for all } x \in \Lambda, \\
& \ominus=\text { the configuration with } \sigma(x)=-1 \text { for all } x \in \Lambda .
\end{aligned}
$$

In the regime (1.4) the Gibbs measure will be concentrated around $\oplus$, which is the unique ground state of $H$. Clearly, $\ominus$ is only a local minimum of $H$, and it is therefore naturally related to metastability.

For $l \in N$, let

$$
E(l)=H\left(\sigma_{l \times l}\right)-H(\ominus),
$$

where $\sigma_{l \times l}$ is the configuration in which the $(+1)$-spins form an $l \times l$ square, centered at the origin, in a sea of $(-1)$-spins. Then $e^{-\beta E(l)}$ is the ratio of the probabilities to see $\sigma_{l \times l}$, respectively, $\ominus$ under the equilibrium $\mu$. It follows from (1.1) that $E(l)=4 J l-h l^{2}$, which is maximal for $l=2 J / h$. This means that, even though an arbitrarily small nonvanishing magnetic field determines the phase, its effect is relevant only on sufficiently large space scales, namely $l \geqslant l_{c}$ with

$$
l_{c}=\left\lceil\frac{2 J}{h}\right\rceil .
$$

Only on such scales the volume energy dominates the surface energy and a larger square of $(+1)$-spins is energetically favorable over a smaller square (see Fig. 1). The choice $h \in(0,2 J)$ corresponds to $l_{c} \in(1, \infty)$, i.e., to a nontrivial critical droplet size.

This describes the metastable behavior from a static point of view.

1.1.2. Glauber dynamics. In order to describe the metastable behavior from a dynamic point of view, we introduce a discrete-time stochastic dynamics by means of a Markov chain on $\mathcal{X}$ with transition probabilities $P\left(\sigma, \sigma^{\prime}\right)$ satisfying the reversibility condition

$$
\mu(\sigma) P\left(\sigma, \sigma^{\prime}\right)=\mu\left(\sigma^{\prime}\right) P\left(\sigma^{\prime}, \sigma\right) \quad \forall \sigma, \sigma^{\prime} \in \mathcal{X},
$$

where $\mu$ is the Gibbs measure in (1.2), and the ergodicity condition

$$
\forall \sigma, \sigma^{\prime} \in \mathcal{X} \quad \exists t \in \mathbb{N} \text { such that } P^{t}\left(\sigma, \sigma^{\prime}\right)>0,
$$

where $P^{t}$ is the $t$-step transition kernel. From the ergodic theorem for reversible Markov chains it follows that $P^{t}\left(\sigma, \sigma^{\prime}\right)$ converges to $\mu\left(\sigma^{\prime}\right)$ as $t \rightarrow \infty$ for all $\sigma, \sigma^{\prime} \in \mathcal{X}$. 
An explicit construction of a Markov chain satisfying the above conditions can be given, for instance, by the Glauber-Metropolis algorithm, which is defined as follows. For $\sigma \in \mathcal{X}$ and $x$ $\in \Lambda$, let

$$
\sigma^{x}(y)= \begin{cases}\sigma(y) & \text { if } x \neq y \\ -\sigma(y) & \text { if } x=y\end{cases}
$$

and choose as transition probabilities

$$
\begin{aligned}
& \sigma \neq \sigma^{\prime}: P\left(\sigma, \sigma^{\prime}\right)=\left\{\begin{array}{l}
0 \quad \text { if } \sigma^{\prime} \neq \sigma^{x} \quad \forall x \in \Lambda \\
\frac{1}{|\Lambda|} e^{-\beta\left[H\left(\sigma^{x}\right)-H(\sigma)\right]_{+}} \quad \text { if } \sigma^{\prime}=\sigma^{x} \exists x \in \Lambda
\end{array}\right. \\
& \sigma=\sigma^{\prime}: P(\sigma, \sigma)=1-\sum_{\sigma^{\prime} \neq \sigma} P\left(\sigma, \sigma^{\prime}\right) .
\end{aligned}
$$

This dynamics randomly selects a site from $\Lambda$ and flips the spin at this site with a probability equal to the Boltzmann weight associated with the positive part of the energy difference caused by the flip. We emphasize that the dynamics given by (1.11) is NC, in the sense that the total magnetization is not a conserved quantity.

1.1.3. Metastability. Suppose we consider the typical paths of the Markov chain defined by (1.11), starting from $\ominus$, in the regime (1.4). We can use a computer simulation and perform a large number of independent runs (see e.g., Tomita and Miyashita ${ }^{3}$ ). What we see is that in the sea of $(-1)$-spins small droplets of $(+1)$-spins appear, which however shrink and disappear before they are able to become large. Only after a very long time, and under the effect of a large fluctuation, a large enough droplet appears that grows without hesitation.

In order to understand this behavior, let us compare the probabilities of shrinking, respectively growing for a connected cluster of $(+1)$-spins in a sea of $(-1)$-spins. First of all, each cluster of $(+1)$-spins becomes rectangular after a finite time (independent of $\beta$ ) with a probability of order one following a sequence of transitions with $H\left(\sigma^{x}\right)-H(\sigma)<0$. Indeed, the rectangle is the only shape such that: (i) all $(+1)$-spins have $\leqslant 2$ nearest-neighbor $(-1)$-spins; (ii) all $(-1)$-spins have $<2$ nearest-neighbor $(+1)$-spins. Hence for the rectangle there are no spins that can be flipped with $H\left(\sigma^{x}\right)-H(\sigma)<0$.

Starting from a rectangular cluster of $(+1)$-spins, to remove a row or column of length 1 costs $(l-1) h$ :

$\left[H\left(\sigma^{x}\right)-H(\sigma)\right]_{+}=[h]_{+}=h$ for each of the sites except the last one.

$\left[H\left(\sigma^{x}\right)-H(\sigma)\right]_{+}=[h-2 J]_{+}=0$ for the last site.

On the other hand, to add a row or column of length 1 costs $2 J-h$ :

$\left[H\left(\sigma^{x}\right)-H(\sigma)\right]_{+}=[2 J-h]_{+}=2 J-h$ for the first site.

$\left[H\left(\sigma^{x}\right)-H(\sigma)\right]_{+}=[-h]_{+}=0$ for each of the sites except the first one.

This means that if the minimal side length 1 of the rectangular cluster is such that $h(l-1)>2 J$ $-h$, i.e., $l \geqslant l_{c}$ with $l_{c}$ given by (1.7), then it tends to grow, while if $l<l_{c}$ then it tends to shrink.

The above heuristic argument has been developed in a rigorous way by Neves and Schonmann $^{4,5}$ (see also Schonmann ${ }^{6-8}$ ). Let $\left(\sigma_{t}\right)_{t \in \mathbb{N}_{0}}$ be the Markov process on $\mathcal{X}$ with transition probabilities as in (1.11). Write $\mathbb{P}_{\sigma}, \mathbb{E}_{\sigma}$ for its probability law and expectation on path space given $\sigma_{0}=\sigma$. Let

$$
\tau_{\sigma}=\min \left\{t \in \mathbb{N}_{0}: \sigma_{t}=\sigma\right\}
$$

be the first hitting time of the configuration $\sigma$. The main result for metastability reads:

Theorem 1.13: (Neves and Schonmann $\left.{ }^{4,5}\right)$ Fix $h \in(0,2 J)$, with $2 J / h$ not integer, put $l_{c}$ $=\lceil 2 \mathrm{~J} / \mathrm{h}\rceil$, and suppose that $\Lambda$ is sufficiently large. 


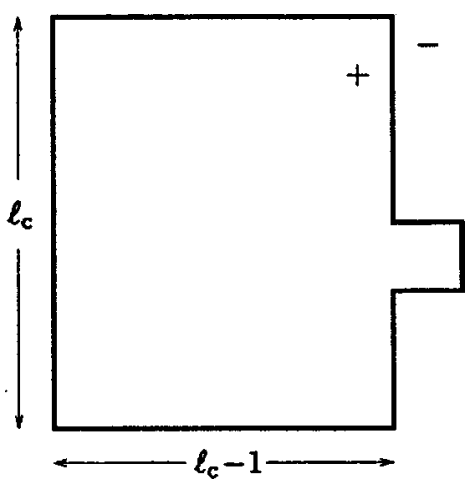

FIG. 2. The critical droplet (NC).

(a) Let $\mathcal{R}$ be the set of configurations where the $(+1)$-spins form a rectangle in a sea of $(-1)$-spins. For $\sigma \in \mathcal{R}$, let $l_{1}(\sigma) \times l_{2}(\sigma)$ be the rectangle of $(+1)$-spins in $\sigma$, and let $l(\sigma)$ $=\min \left\{l_{1}(\sigma), l_{2}(\sigma)\right\}$. Then, for any $\sigma \in \mathcal{R}$,

$$
\begin{aligned}
& l(\sigma)<l_{c}: \lim _{\beta \rightarrow \infty} \mathbb{P}_{\sigma}\left(\tau_{\ominus}<\tau_{\oplus}\right)=1, \\
& l(\sigma) \geqslant l_{c}: \lim _{\beta \rightarrow \infty} \mathbb{P}_{\sigma}\left(\tau_{\oplus}<\tau_{\ominus}\right)=1 .
\end{aligned}
$$

(b) Let $\mathcal{R}^{*}$ be the set of configurations where the $(+1)$-spins form an $l_{c} \times\left(l_{c}-1\right)$ or $\left(l_{c}\right.$ $-1) \times l_{c}$ rectangle with a protuberance attached anywhere to one of the sides of length $l_{c}$. Let $\theta_{\ominus, \oplus}=\max \left\{t<\tau_{\oplus}: \sigma_{t}=\ominus\right\}$ and $\tau_{\ominus, \mathcal{R}^{*}, \oplus}=\min \left\{t>\theta_{\ominus, \oplus}: \sigma_{t} \in \mathcal{R}^{*}\right\}$. Then

$$
\lim _{\beta \rightarrow \infty} \mathrm{P}_{\ominus}\left(\tau_{\ominus, \mathcal{R}^{*}, \oplus}<\tau_{\oplus}\right)=1
$$

(c) Let $\Gamma=\Gamma(J, h)=4 J l_{c}-h\left(l_{c}^{2}-l_{c}+1\right)$. Then

$$
\lim _{\beta \rightarrow \infty} \mathrm{P}_{\ominus}\left(e^{(\Gamma-\delta) \beta}<\tau_{\oplus}<e^{(\Gamma+\delta) \beta}\right)=1 \quad \forall \delta>0
$$

$\mathcal{R}^{*}$ is the set of critical droplets, i.e., the set of saddle points between $\ominus$ and $\oplus$, and $\Gamma(J, h)$ is the formation energy of a critical droplet under the Hamiltonian in (1.1). Theorem 1.13 not only identifies the size and shape of critical droplets (see Fig. 2), it also shows that $\mathcal{R}^{*}$ is the "gate" of the transition from $\ominus$ to $\oplus$ and it identifies the transition time up to logarithmic equivalence in $\beta$.

1.1.4 Nucleation. The problem of identifying the typical path of nucleation, i.e., the path between $\theta_{\ominus, \oplus}$ and $\tau_{\oplus}$, corresponds to the problem of the typical first exit of $\left(\sigma_{t}\right)_{t \in \mathbb{N}_{0}}$ from a suitable region in the state space $\mathcal{X}$. This problem is discussed in detail in Freidlin and Wentzell, ${ }^{9}$ Chap. 6, Schonmann, ${ }^{7}$ Olivieri, and Scoppola, ${ }^{10,11}$ Catoni and Cerf $^{12}$ under rather general hypotheses on the Markov chain. We recall here the main result for the case of the Glauber Ising model.

A sequence of configurations $\sigma_{1}, \ldots, \sigma_{n}(n \in \mathbb{N})$ is called standard when

(1) the (+1)-spins of $\sigma_{i}$ form a rectangular droplet $R_{i}=l_{1, i} \times l_{2, i}$;

(2) $R_{i+1} \backslash R_{i}$ is a single row or a single column;

(3) if $\min \left\{l_{1, i}, l_{2, i}\right\}<l_{c}$, then $\left|l_{1, i}-l_{2, i}\right| \leqslant 1$;

(4) $R_{1}=2 \times 2$ and $R_{n}=\oplus$. 
The configurations in such a sequence are stable, since they are local minima of $H$, i.e., $H\left(\sigma_{i}\right)$ $<\min _{\sigma^{\prime} \sim \sigma_{i}} H\left(\sigma^{\prime}\right)$, where $\sigma^{\prime} \sim \sigma$ if and only if $P\left(\sigma, \sigma^{\prime}\right)>0$. With each $\sigma_{i}$ it is possible to associate a permanence set $\mathcal{Q}_{i}$ (a suitable "environment" of $\sigma_{i}$ : a generalized basin of attraction of $\sigma_{i}$ w.r.t. the dynamics at $\beta=\infty$ ) and a permanence time $T_{i}=\mathbb{E}_{\sigma_{i}} \tau_{\mathcal{Q}_{i}^{c}}$ (the mean exit time of $\mathcal{Q}_{i}$ starting from $\sigma_{i}$ ). In this way we obtain a standard sequence of permanence sets (see Olivieri and Scoppola ${ }^{13}$ for more precise definitions).

For each standard sequence of permanence sets and each $\epsilon>0$ we can introduce a tube of trajectories $\mathbf{T}_{\epsilon, \beta}\left(\mathcal{Q}_{1}, \ldots, \mathcal{Q}_{n}\right)$, defined as the set of paths of configurations visiting the ordered sequence $\mathcal{Q}_{1}, \ldots, \mathcal{Q}_{n}$ and spending in each set $\mathcal{Q}_{i}$ a time that falls in the interval $\left[T_{i} e^{-\epsilon \beta}, T_{i} e^{+\epsilon \beta}\right]$. In terms of these quantities the main result for the path of nucleation reads:

Theorem 1.17: (Schonmann, ${ }^{7}$ Olivieri and Scoppola ${ }^{13}$ ) For every $\kappa, \epsilon>0$ there exists a $\beta_{0}$ $=\beta_{0}(\kappa, \epsilon)$ such that for all $\beta>\beta_{0}$ :

$$
\mathrm{P}_{\ominus}\left(\left(\sigma_{t}\right)_{t \in\left[\theta_{\ominus, \oplus}, \tau_{\oplus}\right]} \in \mathbf{T}_{\epsilon, \beta}\left(\mathcal{Q}_{1}, \ldots, \mathcal{Q}_{n}\right)\right. \text { for some standard }
$$$$
\text { sequence of permanence sets } \left.\mathcal{Q}_{1}, \ldots, Q_{n}\right)>1-e^{-\kappa \beta} \text {. }
$$

Theorem 1.17 shows that the transition from $\ominus$ to $\oplus$ takes place in a narrow tube around rectangular droplets that are squares or quasi-squares when the droplet is subcritical.

The main idea behind Theorem 1.17 (which is actually valid in a much more general context) is the following. The Markov chain $\left(\sigma_{t}\right)_{t \in \mathrm{N}_{0}}$ is in the Freidlin-Wentzell regime, i.e., its state space is finite and its transition probabilities satisfy the following estimates:

$$
e^{-\left[V\left(\sigma, \sigma^{\prime}\right)+\gamma_{\beta}\right] \beta} \leqslant P\left(\sigma, \sigma^{\prime}\right) \leqslant e^{-\left[V\left(\sigma, \sigma^{\prime}\right)-\gamma_{\beta}\right] \beta} \quad \forall \sigma \sim \sigma^{\prime},
$$

where $V(\cdot, \cdot)$ is a non-negative function, and $\lim _{\beta \rightarrow \infty} \gamma_{\beta}=0$. Indeed, this property trivially follows from (1.11), because $\Lambda$ is fixed and $V\left(\sigma, \sigma^{\prime}\right)=\left[H\left(\sigma^{\prime}\right)-H(\sigma)\right]_{+}$. With the help of (1.19) it is standard to obtain estimates on $\mathbb{E}_{\sigma_{i}} \tau_{Q_{i}^{c}}$ and $\mathbb{P}_{\sigma_{i}}\left(\sigma_{\tau_{\mathcal{Q}_{i}^{c}}}=\sigma^{\prime}\right)$ (see Freidlin and Wentzell, ${ }^{9}$ Chap. 6).

The main steps in the proof of Theorem 1.17 are the following:

(1) One must solve a certain sequence of variational problems defined in terms of the energy

function $H$. These variational problems are minimax problems necessary to find the minimal saddle point energy between pairs of states $\sigma, \sigma^{\prime}$ defined by

$$
H\left(\sigma, \sigma^{\prime}\right)=\min \max H(\eta)
$$

where $\phi: \sigma \rightarrow \sigma^{\prime}$ denotes a path from $\sigma$ to $\sigma^{\prime}$. The output of this first step is a standard sequence of configurations.

(2) One must associate with each stable configuration a permanence set and a permanence time. This can be done by using a so-called cycle decomposition: indeed, the permanence sets are generalized cycles. Cycles can be defined in the Freidlin-Wetzell regime (see Freidlin and Wentzell, ${ }^{9}$ Chap. 6, Olivieri and Scoppola, ${ }^{13}$ Trouvé $\left.^{14}\right)$. In the case of the Glauber Ising model cycles turn out to be connected sets of configurations with energy below a given value.

\subsection{The conservative case}

1.2.1. Canonical ensemble. In the present paper we want to study the metastable behavior of conservative systems. To that end we consider a lattice gas model defined as follows. Let $\Lambda_{\beta} \subset \mathbb{Z}^{2}$ be a large finite box centered at the origin, with periodic boundary conditions. With each $x$ $\in \Lambda_{\beta}$ we associate an occupation variable $\eta(x)$, assuming the values 0 or 1 . A lattice gas configuration is denoted by $\eta \in \mathcal{X}=\{0,1\}^{\Lambda_{\beta}}$. We consider the interaction defined by the following Hamiltonian: 


$$
H(\eta)=-U \sum_{(x, y) \in \Lambda_{\beta}^{*}} \eta(x) \eta(y),
$$

where $\Lambda_{\beta}^{*}$ denotes the set of bonds in $\Lambda_{\beta}$, i.e., there is a binding energy $U>0$ between neighboring occupied sites. For $A \subset \Lambda_{\beta}$, we let

$$
N_{A}(\eta)=\sum_{x \in A} \eta(x)
$$

We fix the particle density in $\Lambda_{\beta}$ at

$$
\frac{1}{\left|\Lambda_{\beta}\right|} \sum_{x \in \Lambda_{\beta}} \eta(x)=\rho=e^{-\Delta \beta}
$$

where $\Delta>0$ is an activity parameter. This corresponds to a total number of particles in $\Lambda_{\beta}$ equal to

$$
N=\rho\left|\Lambda_{\beta}\right|
$$

On the set of configurations with $N$ particles

$$
\mathcal{N}_{N}=\left\{\eta \in \mathcal{X}: N_{\Lambda_{\beta}}(\eta)=N\right\}
$$

we define the canonical Gibbs measure

$$
\nu_{N}(\eta)=\frac{e^{-\beta H(\eta)} 1_{\mathcal{N}_{N}}(\eta)}{Z_{N}} \quad(\eta \in \mathcal{X}),
$$

where

$$
Z_{N}=\sum_{\eta \in \mathcal{N}_{N}} e^{-\beta H(\eta)}
$$

We see from (1.23) and (1.24) that in order to have particles at all we must pick $\left|\Lambda_{\beta}\right|$ at least exponentially large in $\beta$. This means that the regime where $\Lambda_{\beta}$ is fixed, considered in the NC-case, has no relevance here. We will in fact be interested in the regime

$$
\Delta \in(U, 2 U), \quad \beta \rightarrow \infty, \quad \lim _{\beta \rightarrow \infty} \frac{1}{\beta} \log \left|\Lambda_{\beta}\right|=\infty,
$$

which takes over the role that (1.4) played in the NC-case.

1.2.2. Kawasaki dynamics. We define a stochastic dynamics in terms of a continuous-time Markov chain $\left(\eta_{t}\right)_{t \geqslant 0}$ with state space $\mathcal{N}_{N}$, given by the following generator:

$$
(\mathcal{L} f)(\eta)=\sum_{(x, y) \in \Lambda_{\beta}^{*}} c((x, y), \eta)\left[f\left(\eta^{(x, y)}\right)-f(\eta)\right]
$$

where

$$
\eta^{(x, y)}(z)= \begin{cases}\eta(z) & \text { if } z \neq x, y \\ \eta(x) & \text { if } z=y \\ \eta(y) & \text { if } z=x\end{cases}
$$


and

$$
c((x, y), \eta)=e^{-\beta\left[H\left(\eta^{(x, y)}\right)-H(\eta)\right]_{+}} .
$$

It is easily verified that the reversibility condition holds:

$$
\nu_{N}(\eta) c((x, y), \eta)=\nu_{N}\left(\eta^{(x, y)}\right) c\left((x, y), \eta^{(x, y)}\right) .
$$

The Markov chain $\left(\eta_{t}\right)_{t \geqslant 0}$ can be represented as follows. With each bond $b=(x, y) \in \Lambda_{\beta}^{*}$ we associate a random clock ringing at exponential times. When the clock at $b$ rings, we consider the configuration with the particles swapped along $b$. This configuration is accepted with a Metropolis rate given by the Boltzmann factor in (1.31). More formally, for each bond $b$ put $\tau_{b, 0}=0$ and let $\tau_{b, i}, i \in \mathbb{N}$, be the sequence of random times whose increments are i.i.d. exponentially distributed with mean 1. Since $\left|\Lambda_{\beta}\right|<\infty$, we have

$$
\mathbb{P}\left(\exists b, b^{\prime}, i, i^{\prime}: \tau_{b, i}=\tau_{b^{\prime}, i^{\prime}}\right)=0 .
$$

Now, if $t=\tau_{b, i}$ for some $b$ and $i$, then we define

$$
\eta_{t}=\left\{\begin{array}{l}
\eta_{t-} \text { with probability } 1-e^{-\beta\left[H\left(\eta_{t-}^{b}\right)-H\left(\eta_{t^{-}}\right)\right]_{+}} \\
\eta_{t-}^{b} \text { with probability } e^{-\beta\left[H\left(\eta_{t-}^{b}\right)-H\left(\eta_{t^{-}}\right)\right]_{+}}
\end{array}\right.
$$

while between ringing times the configuration stays fixed.

1.2.3. Metastability. In order to see that for the regime in (1.28) one can expect metastable behavior, let us consider the grand-canonical Gibbs measure associated with the model, i.e.,

$$
\mu_{\lambda}(\eta)=\frac{e^{-\beta H_{\lambda}(\eta)}}{Z_{\lambda}},
$$

where

$$
H_{\lambda}(\eta)=H(\eta)-\lambda N_{\Lambda_{\beta}}(\eta)
$$

$\lambda \in \mathbb{R}$ is an activity parameter, and

$$
Z_{\lambda}=\sum_{\eta \in \mathcal{X}} e^{-\beta H_{\lambda}(\eta)}
$$

It turns out that if $\lambda=-\Delta$, then for the description of metastability the canonical Gibbs measure is equivalent to the grand-canonical Gibbs measure in the limit of large $\beta$, provided they are suitably restricted in the following way.

Consider the lattice gas at low temperature at its condensation point. Let

$$
\rho_{l}(\beta)=\frac{1+m^{*}(\beta)}{2}, \quad \rho_{g}(\beta)=\frac{1-m^{*}(\beta)}{2}
$$

denote the density of the liquid, respectively, gas phase. Here $m^{*}(\beta)$ is the spontaneous magnetization in the spin language [see (1.44)]. Since

$$
\rho_{g}(\beta)=e^{-2 U \beta}[1+o(1)] \quad(\beta \rightarrow \infty),
$$

we see that $e^{-2 U \beta}$ can be identified as the density of the saturated gas at the condensation point (in the sense of logarithmic equivalence in $\beta$ ). Suppose that we slightly increase the density, avoiding however the appearance of droplets of the liquid phase. Then we get a supersaturated gas that can 


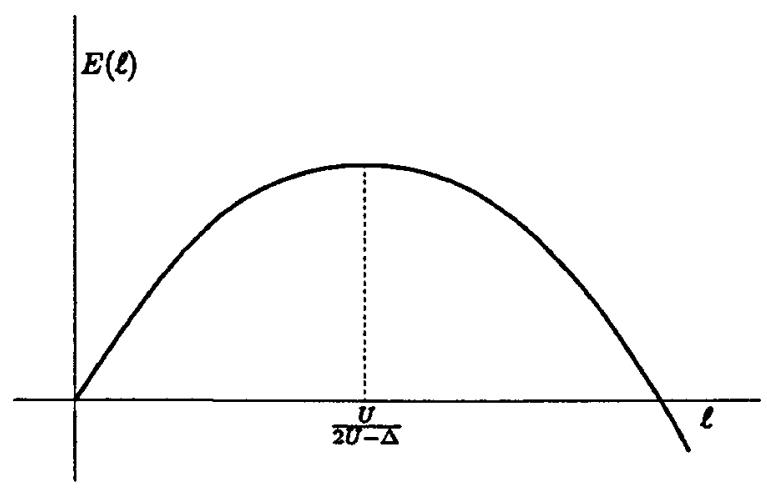

FIG. 3. The energy of an $l \times l$ droplet (C).

be described in terms of a restricted ensemble (see Lebowitz and Penrose ${ }^{15}$ and Capocaccia, Cassandro, and Olivieri ${ }^{16}$ ), namely, the grand-canonical Gibbs measure restricted to a suitable subset of configurations, for instance, where all sufficiently large clusters are suppressed. At low temperature this supersaturated gas will stay rarified, so that its metastable state can be described as a pure gas phase with strong mixing properties.

In these conditions, let us make a rough calculation of the probability to see an $l \times l$ droplet of occupied sites centered at the origin. Under the restricted ensemble, which we denote by $\mu^{*}$, we have

$$
\mu^{*}(l \times l \text { droplet }) \approx \rho^{l^{2}} e^{2 l(l-1) U \beta},
$$

since $\rho$ is the probability to find a particle at a given site and $U$ is the binding energy between particles at neighboring sites. Substituting $\rho=e^{-\Delta \beta}$ we obtain

$$
\mu^{*}(l \times l \text { droplet }) \approx e^{-\beta E(l)},
$$

where

$$
E(l)=2 U l-(2 U-\Delta) l^{2} .
$$

The maximum of $E(l)$ is at $l=U /(2 U-\Delta)$. This means that droplets with side length $l<l_{c}$ have a probability decreasing in 1 and droplets with side length $l \geqslant l_{c}$ a probability increasing in 1 , where

$$
l_{c}=\left\lceil\frac{U}{2 U-\Delta}\right\rceil
$$

(see Fig. 3). The choice $\Delta \in(U, 2 U)$ corresponds to $l_{c} \in(1, \infty)$, i.e., to a non-trivial critical droplet size.

Another way of understanding our choice of $\Delta$ is the following. In the grand-canonical Gibbs measure the configuration can be represented in terms of spin variables. Indeed, after we make the substitution $\eta(x)=[(1+\sigma(x)) / 2]$, where $\sigma(x) \in\{-1,+1\}$ is a spin variable, we can write

$$
\begin{aligned}
H_{\lambda}(\sigma) & =-U \sum_{(x, y) \in \Lambda_{\beta}^{*}} \frac{1+\sigma(x)}{2} \frac{1+\sigma(y)}{2}-\lambda \sum_{x \in \Lambda_{\beta}} \frac{1+\sigma(x)}{2} \\
& =-\frac{U}{4} \sum_{(x, y) \in \Lambda_{\beta}^{*}} \sigma(x) \sigma(y)-\frac{2 U+\lambda}{2} \sum_{x \in \Lambda_{\beta}} \sigma(x)+\text { const. }
\end{aligned}
$$




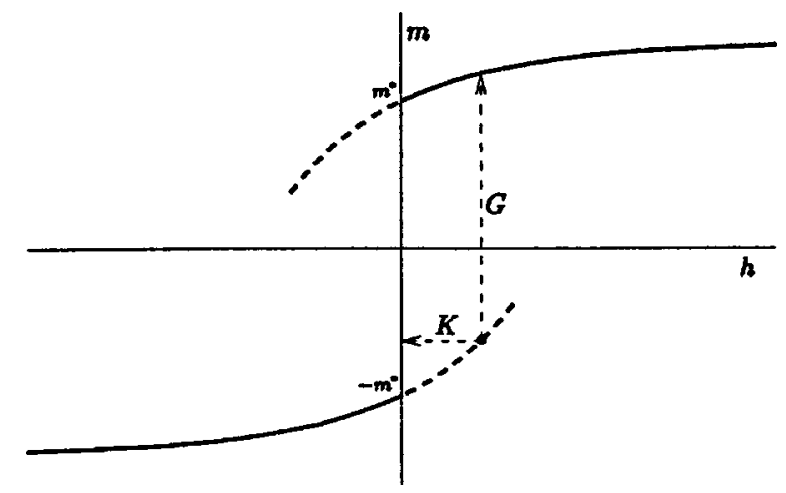

FIG. 4. Relaxation to equilibrium for Glauber (G) and Kawasaki (K) dynamics.

So if $\lambda=-\Delta$, then we have a spin Hamiltonian like (1.1) with pair interaction $J=U / 2$ and magnetic field $h=2 U-\Delta$. By the discussion developed in Sec. 1.1.3, we therefore expect metastable behavior with a critical droplet size given by (1.43) [compare with (1.7)]. The metastable behavior for the NC-case in the spin language occurs when $h \in(0,2 J)$. This corresponds precisely to $\Delta \in(U, 2 U)$.

In physical terms, $\Delta \in(0, U)$ corresponds to the unstable gas, $\Delta=U$ to the spinodal point, $\Delta \in(U, 2 U)$ to the metastable gas, $\Delta=2 U$ to the condensation point, and $\Delta \in(2 U, \infty)$ to the stable gas.

The above describes the metastable behavior from a static point of view. A comparison of Glauber vs Kawasaki dynamics in the spin language is indicated in Fig. 4. In Fig. 4 the boldface dashed lines represent the "metastable branches." In the description with the restricted ensemble there is a specific value of $h$ that corresponds to a canonical metastable state with magnetization $m$. The horizontal dashed line (labeled with $K$ ) represents a Kawasaki transition towards a stable equilibrium with the same global magnetization but with a "segregation" of the two stable pure phases in the equilibrium grand-canonical ensemble at $h=0$ : the saturated gas and the condensed gas (or liquid) at the condensation point.

1.2.4. Local description. Let us now consider the metastable behavior from a dynamic point of view and see what happens locally. As discussed in the NC-case, we want to compare the probabilities of growing, respectively, shrinking for a rectangular cluster of particles. Again the argument will be very rough. Suppose we pick a large finite box $\bar{\Lambda}$, centered at the origin, and start with an $l \times l$ droplet inside $\bar{\Lambda}$. Suppose that the effect on $\bar{\Lambda}$ of the gas in $\Lambda_{\beta} \backslash \bar{\Lambda}$ may be described in terms of the creation of new particles with rate $\rho=e^{-\Delta \beta}$ at sites on the interior boundary of $\Lambda$ and the annihilation of particles with rate 1 at sites on the exterior boundary of $\bar{\Lambda}$. In other words, suppose that inside $\bar{\Lambda}$ the Kawasaki dynamics may be described by a Metropolis algorithm with energy given by the local grand-canonical Hamiltonian:

$$
\bar{H}(\eta)=H(\eta)+\Delta N_{\bar{\Lambda}}(\eta) .
$$

Then the energy barriers for adding, respectively, removing a row or column of length 1 are given in terms of the local saddles of $\bar{H}$ (see Fig. 5):

$$
\begin{array}{ll}
\text { energy barrier for adding } & =2 \Delta-U, \\
\text { energy barrier for removing } & =(2 U-\Delta)(l-2)+2 U,
\end{array}
$$

and the balance of the two barriers indeed gives the critical size $l_{c}$ in (1.43).

Let us briefly discuss the main difficulties arising in the attempt to develop the above idea rigorously and underline the main differences with the NC-case. As we already remarked, in the C-case the Markov chain $\left(\eta_{t}\right)_{t \geqslant 0}$ is not in the Freidlin-Wentzell regime, so we need new ideas. 


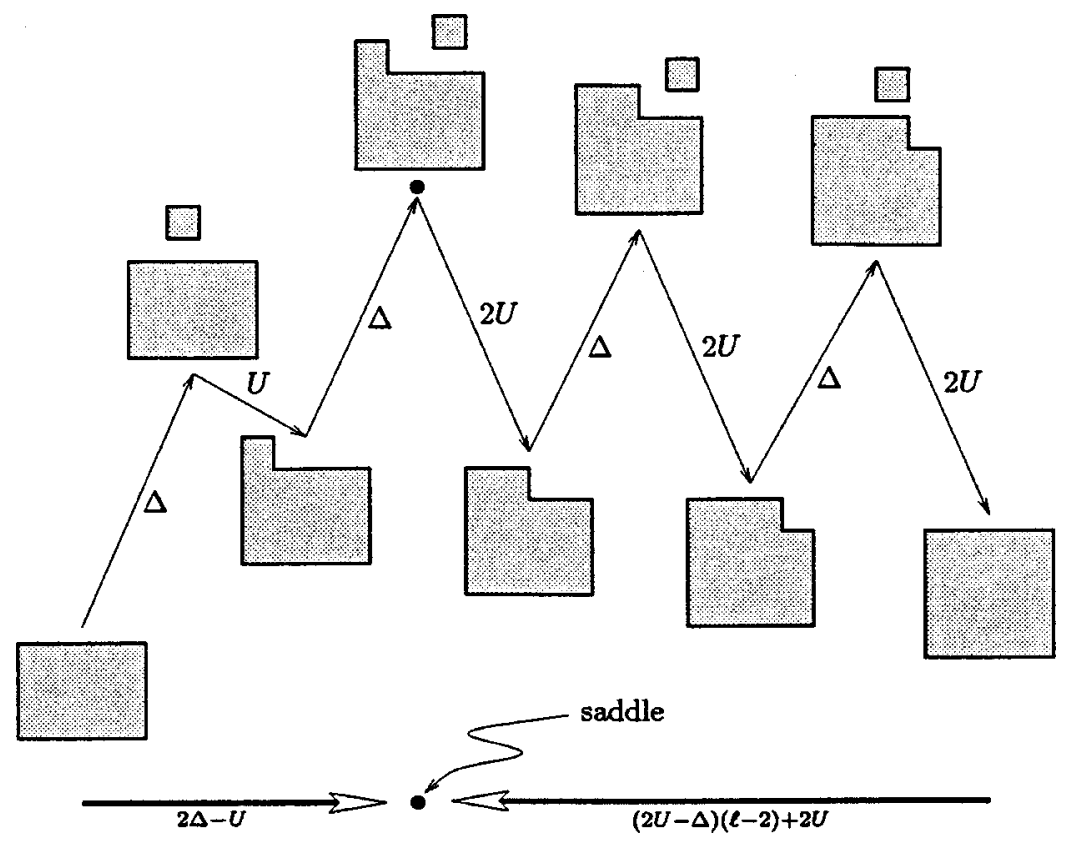

FIG. 5. Local saddles of $\bar{H}$.

The real difficulty is to find the correct way to treat the gas in $\Lambda_{\beta} \backslash \bar{\Lambda}$. The heuristic discussion given above was based on the assumption that the dynamics inside $\bar{\Lambda}$ is effectively described by the local grand-canonical Hamiltonian $\bar{H}$ in (1.45). However, unlike the NC-dynamics, the C-dynamics is not really local: Particles must arrive from or return to the gas, which acts as a reservoir. It is therefore not possible to decouple the dynamics of the particles inside $\bar{\Lambda}$ from the dynamics of the gas in $\Lambda_{\beta} \backslash \bar{\Lambda}$. This means that the gas must be controlled in some detail in order to prove that the above assumption is indeed a good enough approximation.

A second consequence of the non-local behavior of the $\mathrm{C}$-dynamics is that the argument used in the NC-case, based on the stability of configurations and on the corresponding partition into cycles of the state space (see Sec. 1.1.4), is completely lost in the C-case. In other words, we cannot define the stability of a configuration inside $\bar{\Lambda}$, since it depends on the configuration in $\Lambda_{\beta} \backslash \bar{\Lambda}$. A different aspect of the same problem is the following: What is the mechanism by which the gas remains in or close to equilibrium, so that its description in terms of $\bar{H}$ is correct, even over long time intervals during which exchange of many particles occurs?

\subsection{A simplified model}

Unfortunately, we are unable to handle the model described in Sec. 1.2. Instead, in the present paper we solve the problem of metastability for a simplified model. Namely, we remove the interaction outside the box $\bar{\Lambda}_{0}=\bar{\Lambda} \backslash \partial^{-} \bar{\Lambda}$, with $\partial^{-} \bar{\Lambda}$ the interior boundary of $\bar{\Lambda}$, i.e., we replace the interaction energy (1.21) by

$$
H(\eta)=-U \sum_{(x, y) \in \bar{\Lambda}_{0}^{*}} \eta(x) \eta(y) .
$$

Moreover, we also remove the exclusion outside $\bar{\Lambda}$, i.e., the dynamics of the gas outside $\bar{\Lambda}$ is that of independent random walks (IRWs). These two simplifications will allow us to control the gas and to overcome the difficulties outlined in Sec. 1.2.4.

Our state space is 


$$
\mathcal{N}_{N}=\left\{\eta \in \mathcal{X}: N_{\Lambda_{\beta}}(\eta)=N\right\}
$$

where $\mathcal{X}=\{0,1\}^{\bar{\Lambda}} \times \mathbb{N}_{0}^{\Lambda_{\beta} \backslash \bar{\Lambda}}, N_{\Lambda_{\beta}}(\eta)=\Sigma_{x \in \Lambda_{\beta}} \eta(x)$, and $N=\rho\left|\Lambda_{\beta}\right|$ (with $\rho=e^{-\Delta \beta}$ ). The local grand-canonical Hamiltonian is

$$
\bar{H}(\eta)=H(\eta)+\Delta N_{\Lambda}(\eta),
$$

where $H$ is the Hamiltonian in (1.47). Throughout the remainder of this paper we assume that we are in the regime (1.28).

Our main theorem reads as follows. Let

$$
\begin{aligned}
& \square=\left\{\eta \in \mathcal{X}: \eta(x)=1 \quad \forall x \in \bar{\Lambda}_{0}\right\}, \\
& \square=\{\eta \in \mathcal{X}: \eta(x)=0 \quad \forall x \in \bar{\Lambda}\} .
\end{aligned}
$$

For $\bar{\eta} \in \overline{\mathcal{X}}=\{0,1\}^{\bar{\Lambda}}$, let $\nu_{\bar{\eta}}$ denote the canonical Gibbs measure conditioned on the configuration inside $\bar{\Lambda}$ being $\bar{\eta}$, i.e.,

$$
\nu_{\bar{\eta}}(\eta)=\frac{\nu(\eta) 1_{I_{\bar{\eta}}}(\eta)}{\nu\left(I_{\bar{\eta}}\right)} \quad(\eta \in \mathcal{X})
$$

where $I_{\bar{\eta}}=\left\{\eta \in \mathcal{X}:\left.\eta\right|_{\bar{\Lambda}}=\bar{\eta}\right\}$, with $\left.\eta\right|_{\bar{\Lambda}}$ the restriction of $\eta$ to $\bar{\Lambda}$, and $\nu$ is the canonical Gibbs measure defined in (1.24)-(1.27). For $\bar{\eta} \in \overline{\mathcal{X}}=\{0,1\}^{\bar{\Lambda}}$, write $\mathbb{P}_{\nu_{\bar{\eta}}}, \mathbb{E}_{\nu_{\bar{\eta}}}$ to denote the probability law and expectation for the Markov process $\left(\eta_{t}\right)_{t \geqslant 0}$ on $\mathcal{X}$ following the Kawasaki dynamics with Hamiltonian (1.47) given that $\eta_{0}$ is chosen according to $\nu_{\bar{\eta}}$. Write $\bar{\square}$ to denote the empty configuration in $\bar{\Lambda}$, i.e., $\square=I_{\bar{\square}}$. For $\mathcal{A} \subset \mathcal{X}$, let

$$
\tau_{\mathcal{A}}=\min \left\{t \geqslant 0: \eta_{t} \in \mathcal{A}\right\}
$$

be the first hitting time of the set $\mathcal{A}$.

Theorem 1.53: Fix $\Delta \in\left(\frac{3}{2} U, 2 U\right)$, with $U /(2 U-\Delta)$ not integer, put $l_{c}=[U /(2 U-\Delta)]$, and suppose that $\lim _{\beta \rightarrow \infty}(1 / \beta) \log \left|\Lambda_{\beta}\right|=\infty$.

(a) Let $\overline{\mathcal{R}} \subset \overline{\mathcal{X}}$ be the set of configurations inside $\bar{\Lambda}$ where the particles form a square or quasi-square contained in $\bar{\Lambda}_{0}$. For $\bar{\eta} \in \overline{\mathcal{R}}$, let $l_{1}(\bar{\eta}) \times l_{2}(\bar{\eta})$ with $\left|l_{1}(\bar{\eta})-l_{2}(\bar{\eta})\right| \leqslant 1$ be the square or quasi-square of particles in $\bar{\eta}$, and let $l(\bar{\eta})=\min \left\{l_{1}(\bar{\eta}), l_{2}(\bar{\eta})\right\}$. Then, for any $\bar{\eta} \in \overline{\mathcal{R}}$,

$$
\begin{array}{ll}
l(\bar{\eta})<l_{c}: & \lim _{\beta \rightarrow \infty} \mathbb{P}_{\nu_{\bar{\eta}}}\left(\tau_{\square}<\tau_{\square}\right)=1 \\
l(\bar{\eta}) \geqslant l_{c}: & \lim _{\beta \rightarrow \infty} \mathbb{P}_{\nu_{\bar{\eta}}}\left(\tau_{\square}<\tau_{\square}\right)=1 .
\end{array}
$$

(b) Let $\overline{\mathcal{C}}^{*}$ be the set of configurations defined in (4.21) (see Fig. 6 for an example). Let $\theta_{\square, \boldsymbol{\square}}=\max \left\{t<\tau_{\square}: \eta_{t} \in \square\right\}$ and $\tau_{\square, \overline{\mathcal{C}}^{*}, \boldsymbol{\square}}=\min \left\{t>\theta_{\square, \boldsymbol{\square}}: \eta_{t} \in \overline{\mathcal{C}}^{*}\right\}$. Then

$$
\lim _{\beta \rightarrow \infty} \mathbb{P}_{\nu_{\square}}\left(\tau_{\mathbf{m}}, \overline{\mathcal{C}}^{*}, \mathbf{\square}<\tau_{\mathbf{\square}}\right)=1 .
$$

(c) Let $\Gamma=\Gamma(U, \Delta)=-U\left(2 l_{c}^{2}-4 l_{c}+2\right)+\Delta\left(l_{c}^{2}-l_{c}+2\right)$. Then

$$
\lim _{\beta \rightarrow \infty} \mathrm{P}_{\nu_{\square}^{-}}\left(e^{(\Gamma-\delta) \beta}<\tau_{\square}<e^{(\Gamma+\delta) \beta}\right)=1 \quad \forall \delta>0 .
$$




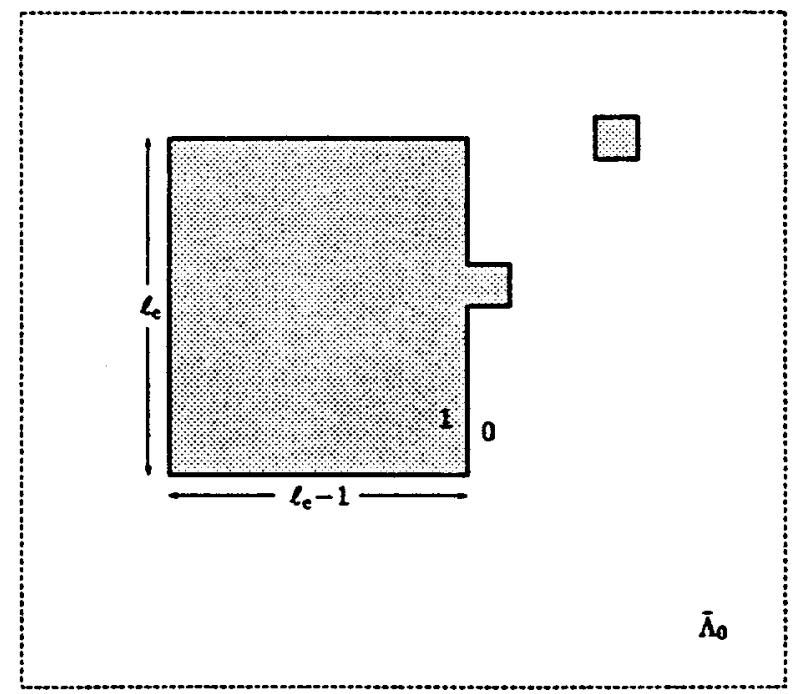

FIG. 6. A critical configuration (C).

Theorem 1.53 is the analogue of Theorem 1.13. There are, however, a number of important differences.

The mechanisms for the evolution of clusters under the Kawasaki dynamics and the Glauber dynamics are different. In particular, under the Kawasaki dynamics there is a movement of particles along the border of a rectangular droplet, leading to a (more stable) square droplet on a time scale much shorter than the one needed to grow or shrink (of order $e^{\Delta \beta}$ ). Moreover, the subcriticality vs supercriticality of a rectangle (i.e., its tendency to reach $\square$ before $\square$ or vice versa) is related to its area. In contrast, under the Glauber dynamics the subcriticality vs supercriticality is related to its minimal side length: a subcritical rectangle is attracted by the maximal square contained in its interior, while a supercritical rectangle does not manifest any tendency towards a square shape.

Let us comment on Theorem 1.53:

Theorem 1.53(a): We only identify the subcriticality vs supercriticality of squares and quasisquares. We believe that it is possible to show that, starting from an $l_{1} \times l_{2}$ rectangle that is not square or quasi-square, the system forms a square or quasi-square with volume $>l_{1} l_{2}$ in a time of order $e^{\Delta \beta}$ and from there proceeds as described in (1.54).

Theorem 1.53(b): $\overline{\mathcal{C}}^{*}$ is the set of critical droplets, i.e., the set of saddle points between $\square$ and $\mathbf{\square}$, that form the "gate" of the transition from $\square$ to $\mathbf{\square}$. Let $\overline{\mathcal{R}}^{*} \subset \overline{\mathcal{X}}$ be the set of configurations inside $\bar{\Lambda}$ where the particles form an $l_{c} \times\left(l_{c}-1\right)$ or $\left(l_{c}-1\right) \times l_{c}$ quasi-square with a protuberance attached anywhere to one of the sides of length $l_{c}$ and with a free particle anywhere else, all contained in $\bar{\Lambda}_{0}$ (see Fig. 6). We will see in Sec. 4.2 that $\overline{\mathcal{C}}^{*}$ consists of all configurations that are "U-equivalent" to some configuration in $\overline{\mathcal{R}}^{*}$, i.e., have the same energy and can be connected via a path with a "maximal saddle U." In particular, $\overline{\mathcal{C}}^{*} \supset \overline{\mathcal{R}}^{*}$, but the full set is more complex (see Fig. 9 in Sec. 5.2). This complexity comes from the fact that under the Kawasaki dynamics particles can move along the border of a rectangular droplet at a cost $\mathrm{U}$.

Theorem 1.53(c): $\Gamma(U, \Delta)$ is the energy of a critical droplet under the local grand-canonical Hamiltonian in (1.49).

The critical configuration in Fig. 6 has the same shape as in the NC-case (see Fig. 2), but with an extra free particle. This particle signals that the "gate" of the transition from $\square$ to $\mathbf{\square}$ has been passed and that the droplet starts to grow without hesitation.

It is certainly feasible to also prove the analogue of Theorem 1.17 for the simplified model. However, in the present paper we will not address this issue for reasons of space. 


\section{Remarks:}

(1) Our proof of Theorem 1.53 shows that the convergence in (1.54)-(1.56) is exponentially fast in $\beta$.

(2) As explained above, the removal of the interaction outside $\bar{\Lambda}_{0}$ and the exclusion outside $\bar{\Lambda}$ allows us to mathematically control the gas. From a physical point of view this approximation seems very reasonable, because $\beta \rightarrow \infty$ corresponds to a low density limit $\left(\rho=e^{-\Delta \beta}\right)$ in which the gas essentially behaves like an ideal gas.

(3) In the simplified model we are focusing on the local aspects of metastability and nucleation: the removal of the interaction outside $\bar{\Lambda}_{0}$ forces the critical droplet to appear inside $\bar{\Lambda}_{0}$. In the original model with interaction and exclusion throughout $\Lambda_{\beta}$, if $\lim _{\inf } \operatorname{lom}_{\beta \rightarrow \infty}(1 / \beta) \log \left|\Lambda_{\beta}\right|$ is large enough, then the decay from the metastable to the stable state is driven by the formation of many droplets far away from the origin, which subsequently grow, coalesce and reach $\bar{\Lambda}_{0}$. This is a much harder problem, which we hope to tackle in the future (see Deghampour and Schonmann ${ }^{17}$ for a description of this behavior for Ising spins under Glauber dynamics). Also, in the original model the question of the growth of large supercritical droplets comes up, which is absent for the simplified model because $\bar{\Lambda}_{0}$ is finite. For Kawasaki dynamics this poses new problems compared to Glauber dynamics, because large droplets deplete the gas.

\subsection{Outline of the paper}

Our strategy to prove Theorem 1.53 will be the following. In Sec. 2 we show that, under the measure $\nu_{\bar{\eta}}(\bar{\eta} \in \overline{\mathcal{X}})$, particle densities in suitable regions around $\bar{\Lambda}$ are not too far from their expected value. With the help of large deviation estimates we show that these density properties are preserved under the dynamics over very long time intervals with a very large probability. In Sec. 3 we use this fact to control the gas, essentially via a series of mixing propositions. Once the gas behavior is under control, we start to tackle the metastability problem inside $\bar{\Lambda}$. This is done in Secs. 6-7 via recurrence and reduction. Namely, in Sec. 6 we show that certain subsets of configurations of increasing "regularity"

$$
\mathcal{X}_{1} \supset \mathcal{X}_{2} \supset \mathcal{X}_{3}
$$

are visited by the process on certain basic time scales

$$
T_{1}=e^{0 \beta} \ll T_{2}=e^{U \beta} \ll T_{3}=e^{\Delta \beta} .
$$

This fact leads us in Sec. 7 to define a reduced Markov chain with state space $\mathcal{X}_{3}$, whose transition probabilities we can estimate in a way that allows us to control the metastable behavior. In essence, we show that this reduced chain is "equivalent" in its metastable behavior to a local Markov chain with state space $\overline{\mathcal{X}}=\{0,1\}^{\bar{\Lambda}}$ that is reversible w.r.t. the local grand-canonical Hamiltonian $\bar{H}$ defined in (1.49). This approximation is what drives the argument. In Sec. 5 we study the local Markov chain using general ideas from renormalization. The dynamics inside $\bar{\Lambda}$ is still conservative, and this difficulty has to be handled via local geometric arguments, as explained in Sec. 4. Here we also show that the Kawasaki dynamics has its own peculiarities, which need to be understood in order to describe the evolution of droplets. The proof of our main result in Theorem 1.53 comes in Sec. 8. Here the fact that the full Markov chain is reversible w.r.t. the canonical Gibbs measure plays an important role. In the Appendix we prove the equivalence of the canonical and the grand-canonical ensemble for the simplified model in the regime (1.28). This equivalence is used in some of the calculations.

\subsection{Additional notation}

Before we start the proof of Theorem 1.53, we collect some additional notation beyond (1.47)-(1.52). 
We use capital letters for subsets of $\mathbb{Z}^{2}$, calligraphic capital letters for subsets of the configuration space $\mathcal{X}$, and boldface capital letters for events involving the Markov process and the clocks. This style is used consistently in order to keep different types of quantities apart. We use the symbols $t, T$ for time, $\nu$ for the canonical Gibbs measure with particle density $\rho=e^{-\Delta \beta}$ [recall (1.24) $-(1.27)]$, and $\kappa$ for a generic positive constant.

For $A \subset \mathbb{Z}^{2}$, the set of (nearest-neighbor) bonds in $A$ is

$$
A^{*}=\{b=(x, y): x, y \in A\} .
$$

For $\mathcal{A} \subset \mathcal{X}$, the base of $\mathcal{A}$ is

$$
\operatorname{BASE}(\mathcal{A})=\min \left\{A \subset \mathbb{Z}^{2}: \eta \in \mathcal{A} \Rightarrow\left(\zeta \in \mathcal{A} \forall \zeta \text { such that }\left.\zeta\right|_{A}=\left.\eta\right|_{A}\right)\right\},
$$

i.e., the minimal set of sites on which the configuration determines the event $\mathcal{A}$. For $A \subset \mathbb{Z}^{2}$, the interior resp. exterior boundary of $A$ are

$$
\begin{aligned}
& \partial^{-} A=\{x \in A: \exists b=(x, y): y \notin A\}, \\
& \partial^{+} A=\{x \notin A: \exists b=(x, y): y \in A\} .
\end{aligned}
$$

For $l \in \mathbb{N}$, the box with side length 1 centered at the origin is denoted by $\Lambda_{l}$. The side length of $\bar{\Lambda}_{0}$, the local box appearing in the Hamiltonian $H$ in (1.47), is $l_{0}$. We assume that $l_{0} \gg l_{c}$, the critical droplet size defined in (1.43).

All quantities that live on $\bar{\Lambda}$ are written with a bar on top, in order to distinguish them from quantities that live on $\Lambda_{\beta}$ or other boxes. A function $\beta \mapsto f(\beta)$ is called superexponentially small (SES) if

$$
\lim _{\beta \rightarrow \infty} \frac{1}{\beta} \log f(\beta)=-\infty
$$

We frequently round off large integers, in order to avoid a plethora of brackets like $\lceil\cdot\rceil$.

\section{LD-ESTIMATES FOR CLOCKS AND EQUILIBRIUM}

In this section we formulate several large deviation estimates that will be needed later on.

\subsection{LD-estimates for clocks}

Let $\tau_{b, i}, i \in \mathbb{N}$, denote the ringing times of the clock at bond $b$. For $t>0$, let $r_{b}(t)=\max \{i$ $\left.\in \mathbb{N}: \tau_{b, i} \leqslant t\right\}$ denote the number of rings prior to time $t$. For $m, n \in \mathbb{N}$, put $r_{b}(n, n+m)=r_{b}(n$ $+m)-r_{b}(n)$. For $A \subset \mathbb{Z}^{2}, T \geqslant 0$ and $\delta>0$, define

$$
\mathbf{R}_{T}^{\delta}(A)=\left\{\forall b \in A^{*} \quad \forall n \leqslant T \quad \forall m \geqslant e^{\delta \beta}: r_{b}(n, n+m) \in\left[\frac{1}{2} m, \frac{3}{2} m\right]\right\} .
$$

Proposition 2.2 below shows that clocks ring regularly over long time intervals. This proposition will be needed to switch from continuous to discrete time.

Proposition 2.2: For all $A \subset \mathbb{Z}^{2}, T \geqslant 0$ and $\delta>0$ :

$$
\mathrm{P}\left(\mathbf{R}_{T}^{\delta}(A)^{c}\right) \leqslant T\left|A^{*}\right| \mathrm{SES} .
$$

Proof: Write

$$
\begin{aligned}
\mathbb{P}\left(\mathbf{R}_{T}^{\delta}(A)^{c}\right) & =\left\{\exists b \in A^{*} \quad \exists n \leqslant T \quad \exists m \geqslant e^{\delta \beta}: r_{b}(n, n+m) \notin\left[\frac{1}{2} m, \frac{3}{2} m\right]\right\} \\
& \leqslant T\left|A^{*}\right| \sum_{m \geqslant e^{\delta \beta}} \mathbb{P}\left(r_{b_{0}}(0, m) \notin\left[\frac{1}{2} m, \frac{3}{2} m\right]\right),
\end{aligned}
$$


where $b_{0}$ is any given bond. We have

$$
\begin{aligned}
& r_{b_{0}}(0, m)<\frac{1}{2} m \Rightarrow \tau_{b_{0},\left\lceil\frac{1}{2} m_{\rceil}>m,\right.}, \\
& r_{b_{0}}(0, m)>\frac{3}{2} m \Rightarrow \tau_{b_{0},\left\lceil\frac{3}{2} m\right\rceil}<m .
\end{aligned}
$$

Since $\tau_{b_{0}, m}=\mathcal{X}_{1}+\cdots+\mathcal{X}_{m}$, with $\left(\mathcal{X}_{i}\right)_{i \in \mathbb{N}}$ i.i.d. exponential random variables with mean 1 , a standard LD-estimate gives that the summand of the last term in (2.4) is $\leqslant e^{-\kappa m}$ for some $\kappa$ $>0$. Hence the claim follows. QED

\subsection{LD-estimates for equilibrium}

2.2.1. Hitting times. Proposition 2.6 below gives us an estimate on the hitting time, under the dynamics starting in equilibrium, of sets that have a small probability under the equilibrium measure.

Proposition 2.6. Let $\mathcal{A} \subset \mathcal{X}$ and $\tau_{\mathcal{A}}=\inf \left\{s \geqslant 0: \eta_{s} \in \mathcal{A}\right\}$. Then, for any $t \geqslant 0$,

$$
\mathbb{P}_{\nu}\left(\tau_{\mathcal{A}}<t\right)=\sum_{\eta \in \mathcal{X}} \nu(\eta) \mathrm{P}_{\eta}\left(\tau_{\mathcal{A}}<t\right) \leqslant 3 t|\operatorname{BASE}(\mathcal{A}) *| \nu(\mathcal{A}) .
$$

The same holds when $\nu$ is replaced by $\nu_{\mathcal{B}}=\nu 1_{\mathcal{B}} / \nu(\mathcal{B})$ for any $\mathcal{B} \subset \mathcal{X}$.

Proof: Fix $\mathcal{A}$. For $s \geqslant 0$, let

$$
\mathbf{F}_{s}=\left\{\eta_{s} \in \mathcal{A}, \eta_{u} \notin \mathcal{A} \forall 0 \leqslant u<s\right\} .
$$

Fix $\epsilon>0$ and define

$$
\mathbf{R}_{s}=\{\text { some clock in } \operatorname{BASE}(\mathcal{A}) * \text { rings during }[s, s+\epsilon)\} .
$$

Then we have

$$
\mathbb{P}_{\nu}\left(\tau_{\mathcal{A}}<t\right)=\mathbb{P}_{\nu}\left(\exists s \in[0, t): \mathbf{F}_{s}\right)=\mathbb{P}_{\nu}\left(\exists s \in[0, t): \mathbf{F}_{s} \cap \mathbf{R}_{s}\right)+\mathbb{P}_{\nu}\left(\exists s \in[0, t): \mathbf{F}_{s} \cap \mathbf{R}_{s}^{c}\right) .
$$

The first term equals $\mathbb{P}_{\nu}\left(\tau_{\mathcal{A}}<t\right)\left[1-e^{-\epsilon\left|\operatorname{BASE}(\mathcal{A})^{*}\right|}\right]$, because clocks have no memory. The second term is bounded above by

$$
\mathbb{P}_{\nu}\left(\exists 0<i \leqslant t / \epsilon: \eta_{i \epsilon} \in \mathcal{A}\right) \leqslant \frac{t}{\epsilon} \nu(\mathcal{A}),
$$

where we use that $\mathbb{P}_{\nu}\left(\eta_{i \epsilon} \in \mathcal{A}\right)$ does not depend on $i$ because $\nu$ is the equilibrium measure. Combining the latter two observations with (2.10) we get

$$
\mathrm{P}_{\nu}\left(\tau_{\mathcal{A}}<t\right) \leqslant t \nu(\mathcal{A})\left[\frac{1}{\epsilon} e^{\epsilon|\operatorname{BASE}(\mathcal{A}) *|}\right] .
$$

Optimize over $\epsilon$, i.e., pick $\epsilon=1 /\left|\operatorname{BASE}(\mathcal{A})^{*}\right|$, to arrive at the claim. QED

2.2.2. Recurrence times. Proposition 2.13 gives us control over the successive times at which the dynamics hits a certain set. This proposition will be needed later on to establish recurrence properties to certain special sets.

Proposition 2.13: Let $T \leqslant T^{\prime} \leqslant T^{\prime \prime}$ and let $\mathcal{A}, \mathcal{B} \subset \mathcal{X}$. Suppose that there exists an event $\mathbf{E}_{T} \subset \mathcal{X}^{[0, T)}$ such that

$$
\begin{aligned}
& \text { (i) } \mathbf{E}_{T} \subset\left\{\exists t \in[0, T): \eta_{t} \in \mathcal{A}\right\}, \\
& \text { (ii) } \min _{\eta_{0} \in \mathcal{B}} \mathrm{P}_{\eta_{0}}\left(\mathbf{E}_{T}\right) \geqslant p>0 .
\end{aligned}
$$


Then

$$
\mathrm{P}_{\nu}\left(\exists t \in\left[0, T^{\prime \prime}\right): \eta_{s} \notin \mathcal{A} \quad \forall s \in\left[t, t+T^{\prime}\right)\right) \leqslant T^{\prime \prime}\left[3\left|\operatorname{BASE}\left(\mathcal{B}^{c}\right)^{*}\right| \nu\left(\mathcal{B}^{c}\right)+(1-p)^{T^{\prime} / T}\right] .
$$

Proof: Pick any $t \in\left[0, T^{\prime \prime}\right)$. Split the time interval $\left[t, t+T^{\prime}\right)$ into pieces of length $T$. By (2.14) (i)-(ii), on the event $\left\{\tau_{\mathcal{B}^{c}} \geqslant T^{\prime \prime}\right\}$, if at the beginning of a piece the process is not in $\mathcal{A}$, then it has a probability at most $1-p$ not to enter $\mathcal{A}$ during this piece. Hence the probability not to enter $\mathcal{A}$ during the time interval $\left[t, t+T^{\prime}\right)$ is at most $(1-p)^{T^{\prime} / T}$ by the Markov property. Consequently,

$$
\mathbb{P}_{\nu}\left(\exists t \in\left[0, T^{\prime \prime}\right): \eta_{s} \notin \mathcal{A} \quad \forall s \in\left[t, t+T^{\prime}\right)\right) \leqslant \mathbb{P}_{\nu}\left(\tau_{\mathcal{B}^{c}}<T^{\prime \prime}\right)+T^{\prime \prime}(1-p)^{T^{\prime} / T} .
$$

Now use Proposition 2.6 to get the claim. QED

2.2.3. Particle density in annuli around $\bar{\Lambda}$. Propositions $2.17,2.20$, and 2.23 below give us control over the number of particles in annuli around $\bar{\Lambda}$ with a side length that is close to the mean particle distance on an exponential scale. In the proofs we compute the estimates using the grand-canonical Gibbs measure $\mu$ on $\mathbb{Z}^{2}$ with particle density $\rho$, rather than the canonical Gibbs measure $\nu$ on $\Lambda_{\beta}$ with total particle number $\rho\left|\Lambda_{\beta}\right|$. However, by the equivalence of ensembles proved in the Appendix, the difference is SES under our assumption that $\lim _{\beta \rightarrow \infty}(1 / \beta) \log \left|\Lambda_{\beta}\right|$ $=\infty$ (see the remark at the end of the Appendix).

Proposition 2.17: Let $\gamma>0$ and $l_{+}=e^{(1 / 2)(\Delta+\gamma) \beta}$. Then, for all $\gamma^{\prime} \in(0, \gamma)$,

$$
\nu\left(\left\{\eta \in \mathcal{X}: N_{\Lambda_{l_{+}} \backslash \bar{\Lambda}_{0}}(\eta) \leqslant e^{\gamma^{\prime} \beta}\right\}\right)=\operatorname{SES} .
$$

Proof: Abbreviate $M=e^{\gamma^{\prime} \beta}$. Let $\mathcal{A}=\left\{\eta \in \mathcal{X}: N_{\Lambda_{l_{+}} \backslash \bar{\Lambda}}(\eta) \leqslant M\right\}$. Then

$$
\mu(\mathcal{A}) \leqslant e^{M} \sum_{\eta \in \mathcal{X}} \mu(\eta) e^{-N_{\Lambda_{l_{+}}} \backslash \bar{\Lambda}(\eta)}=e^{M}\left[e^{-\left(1-e^{-1}\right) \rho}\right]^{\left|\Lambda_{l_{+}} \backslash \bar{\Lambda}\right|}=e^{M}(1+o(1)) \exp \left[-\left(1-e^{-1}\right) e^{\gamma \beta}\right],
$$

where we use that $\mu$ outside $\bar{\Lambda}$ places particles according to a Poisson random field with density $\rho$, and we note that $\left|\Lambda_{l_{+}}\right|=e^{\gamma \beta} / \rho$. QED

Proposition 2.20: Let $\gamma>0$ and $l_{-}=e^{(1 / 2)(\Delta-\gamma) \beta}$. Then

$$
\nu\left(\left\{\eta \in \mathcal{X}: N_{\Lambda_{l_{-}} \backslash \bar{\Lambda}_{0}}(\eta) \geqslant \log \beta\right\}\right)=\operatorname{SES} .
$$

Proof: Abbreviate $M=\log \beta$. Let $\mathcal{A}=\left\{\eta \in \mathcal{X}: N_{\Lambda_{l_{-}} \backslash \bar{\Lambda}}(\eta) \geqslant M\right\}$. Then

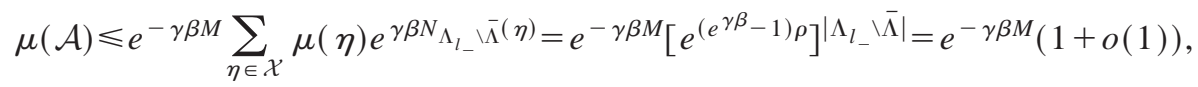

where we note that $\left|\Lambda_{l_{-}}\right|=e^{-\gamma \beta} / \rho$. QED

Proposition 2.23: Let $\gamma>0$ and $l_{-}=e^{(1 / 2)(\Delta-\gamma) \beta}$. Then, for all $n \in \mathbb{N}$,

$$
\nu\left(\left\{\eta \in \mathcal{X}: N_{2^{n+1} \Lambda_{l_{-}} \backslash 2^{n} \Lambda_{l_{-}}}(\eta) \geqslant\left(2^{n+1}-2^{n}\right)^{2} \log \beta\right\}\right)=(\operatorname{SES})^{2^{2 n}} .
$$

Proof: Abbreviate $M=\log \beta$. For $n \in \mathbb{N}$, let $\mathcal{A}_{n}=\left\{\eta \in \mathcal{X}: N_{2^{n+1} \Lambda_{l_{-}} \backslash 2^{n} \Lambda_{l_{-}}}(\eta) \geqslant\left(2^{n+1}\right.\right.$ $\left.\left.-2^{n}\right)^{2} M\right\}$. The same estimate as in (2.22) gives 


$$
\mu\left(\mathcal{A}_{n}\right) \leqslant e^{-\gamma\left(2^{n+1}-2^{n}\right)^{2} \beta \log \beta}(1+o(1)) \quad(n \in \mathbb{N})
$$

with the error term uniform in $n$. QED

Define

$$
\begin{gathered}
\mathcal{X}_{0}^{1}=\left\{\eta \in \mathcal{X}: N_{\Lambda_{l_{+}} \backslash \bar{\Lambda}}(\eta)>e^{\gamma^{\prime} \beta}\right\}, \\
\mathcal{X}_{0}^{2}=\left\{\eta \in \mathcal{X}: N_{\Lambda_{l_{-}} \backslash \bar{\Lambda}}(\eta)<\log \beta\right\}, \\
\mathcal{X}_{0}^{3, n}=\left\{\eta \in \mathcal{X}: N_{\left.2^{n+1} \Lambda_{l_{-} \backslash 2^{n} \Lambda_{l_{-}}}(\eta)<\left(2^{n+1}-2^{n}\right)^{2} \log \beta\right\},}\right.
\end{gathered}
$$

and put

$$
\mathcal{X}_{0}=\mathcal{X}_{0}^{1} \cap \mathcal{X}_{0}^{2} \cap\left\{\begin{array}{l}
\bigcap \mathcal{X}_{0}^{3, n} \\
n \in \mathbb{N}
\end{array}\right\} .
$$

Proposition 2.28: Let $\mathbf{A}_{T}=\left\{\eta_{t} \in \mathcal{X}_{0} \quad \forall t \in[0, T)\right\}$. Then

$$
\mathrm{P}_{\nu}\left(\mathbf{A}_{T}^{c}\right)=\mathrm{SES} \text { for all } T \leqslant e^{C \beta} \text { with } C \text { arbitrarily large. }
$$

Proof: Estimate

$$
\mathbb{P}_{\nu}\left(\mathbf{A}_{T}^{c}\right) \geqslant \mathbb{P}_{\nu}\left(\tau_{\left(\mathcal{X}_{0}^{1}\right)^{c}}<T\right)+\mathbb{P}_{\nu}\left(\tau_{\left(\mathcal{X}_{0}^{2}\right)^{c}}<T\right)+\sum_{n \in \mathbb{N}} \mathbb{P}_{\nu}\left(\tau_{\left(\mathcal{X}_{0}^{3, n}\right)^{c}}<T\right)
$$

and use Proposition 2.6 in combination with Propositions 2.17, 2.20, and 2.23. Here note that $\left|\operatorname{BASE}\left(\left(\mathcal{X}_{0}^{1}\right)^{c}\right)^{*}\right|,\left|\operatorname{BASE}\left(\left(\mathcal{X}_{0}^{2}\right)^{c}\right)^{*}\right|$ and $2^{-2 n}\left|\operatorname{BASE}\left(\left(\mathcal{X}_{0}^{3, n}\right)^{c}\right)^{*}\right|$ grow only exponentially fast with $\beta$. QED

Proposition 2.28 will be crucial later on. Namely, it says that over the exponentially long intervals we are considering for the metastable behavior we may as well assume that the process $\left(\eta_{t}\right)_{t \geqslant 0}$ never leaves $\mathcal{X}_{0}$. The set $\mathcal{X}_{0}$ consists of those configurations where the gas outside $\bar{\Lambda}$ is "close to equilibrium.",

\section{LD-ESTIMATES FOR INDEPENDENT RANDOM WALKS}

In this section we formulate several large deviation estimates that involve hitting times for particles performing independent random walks. We do the estimates pretending that the random walks live on $\mathbb{Z}^{2}$ instead of $\Lambda_{\beta}$. However, this only causes an error that is SES because of our assumption that $\lim _{\beta \rightarrow \infty}(1 / \beta) \log \left|\Lambda_{\beta}\right|=\infty$.

\subsection{LD-estimates for a single random walk}

\subsubsection{Hitting times. Let}

$$
\left(\xi_{t}\right)_{t \geqslant 0}
$$

be a simple random walk on $\mathbb{Z}^{2}$ with jump rate 1 . Let $\mathbb{P}_{x}$ denote its law on path space given $\xi_{0}$ $=x$. Let $\tau_{\bar{\Lambda}}=\min \left\{t \geqslant 0: \xi_{t} \in \bar{\Lambda}\right\}$. Proposition 3.2 below gives us control over $\tau_{\bar{\Lambda}}^{-}$when the random walk starts from $x \in \partial^{+} \bar{\Lambda}$.

Proposition 3.2: There exist $\kappa>0$ and $t_{0}>0$ such that, for all $t>t_{0}$,

$$
\min _{x \in \partial^{+}{ }^{-}} \mathbb{P}_{x}\left(\tau_{\Lambda}^{-}>t\right) \geqslant \frac{\kappa}{\log t} .
$$


Proof: We begin by proving the analogous estimate for discrete time.

(1) Let $\left(\xi_{n}\right)_{n \in N_{0}}$ be a simple random walk on $\mathbb{Z}^{2}$. Let $\tau_{\Lambda}^{-}=\min \left\{n>0: \xi_{n} \in \bar{\Lambda}\right\}$ (which does not include $n=0$ ), and put

$$
\theta_{n}^{\bar{\Lambda}}=\max \left\{0 \leqslant m \leqslant n: \xi_{m} \in \bar{\Lambda}\right\} .
$$

Pick $x \in \bar{\Lambda}$ and write

$$
1=\sum_{m=0}^{n} \mathbb{P}_{x}\left(\theta_{n}^{\bar{\Lambda}}=m\right)=\sum_{m=0}^{n} \sum_{y \in \partial^{-} \bar{\Lambda}} \mathbb{P}_{x}\left(\xi_{m}=y\right) \mathbb{P}_{y}\left(\tau_{\Lambda}^{-}>n-m\right) .
$$

Split the sum over $m$ into two parts: $0 \leqslant m \leqslant n[1-(1 / \log n)]$ and the rest. The first part can be bounded above by

$$
\left|\partial^{-} \bar{\Lambda}\right|\left[1+\sum_{m=1}^{n[1-(1 / \log n)]} \frac{\kappa_{1}}{m}\right]_{y \in \partial^{-} \bar{\Lambda}} \max _{y}\left(\tau_{\Lambda}^{-}>\frac{n}{\log n}\right),
$$

where we use that

$$
\max _{z \in \mathbb{Z}^{2}} \mathbb{P}_{0}\left(\xi_{m}=z\right) \leqslant \frac{\kappa_{1}}{m} \quad \forall m \geqslant 1
$$

(see Spitzer ${ }^{18}$ Sec. 7). The second part can be bounded above by

$$
\left|\partial^{-} \bar{\Lambda}\right| \sum_{m=n[1-(1 / \log n)]+1}^{n} \frac{\kappa_{1}}{m} .
$$

Combining the two bounds in (3.6) and (3.8) with (3.5), we obtain, for $n$ large enough,

$$
\max _{y \in \partial^{-} \bar{\Lambda}} \mathbb{P}_{y}\left(\tau_{\Lambda}^{-}>\frac{n}{\log n}\right) \geqslant \frac{\kappa_{2}}{\log n} .
$$

(2) Since any two sites in $\partial^{-} \bar{\Lambda}$ can be connected by a path outside $\bar{\Lambda}$ of length at most $2\left(l_{0}+2\right)$, it follows that, uniformly in $n$,

$$
\min _{y \in \partial^{-} \bar{\Lambda}^{-}} \mathbb{P}_{y}\left(\tau_{\Lambda}^{-}>\frac{n}{\log n}\right) \geqslant \kappa_{3} \max _{y \in \partial^{-} \bar{\Lambda}} \mathbb{P}_{y}\left(\tau_{\Lambda}^{-}>\frac{n}{\log n}-\kappa_{4}\right) .
$$

Together with (3.9) this gives

$$
\min _{x \in \partial^{-} \bar{\Lambda}} \mathbb{P}_{x}\left(\tau_{\Lambda}^{-}>\frac{n}{\log n}\right) \geqslant \frac{\kappa_{5}}{\log n},
$$

which implies (3.3) for discrete time after replacing $n$ by $n \log n$.

(3) The extension to continuous time is trivial, via a standard LD-estimate on the clock of the random walk. QED

The bound in Proposition 3.2 decays very slowly with $t$ because SRW on $\mathbb{Z}^{2}$ is only marginally recurrent. This slow decay will be useful later on in estimates of probabilities of various events where we want to keep particles away from $\bar{\Lambda}$.

3.1.2. Trapping times: Let $A$ be a rectangular subset of $Z^{2}$. Let 


$$
\left(\hat{\xi}_{t}^{A}\right)_{t \geqslant 0}
$$

be a simple random walk on $\mathbb{Z}^{2} \backslash A$ with jump rate 1 with the property that when it hits $\partial^{+} A$ it gets "trapped," in the sense that a step from $\partial^{+} A$ to $\partial^{++} A$, the exterior boundary of $A \cup \partial^{+} A$, occurs at rate $e^{-U \beta}$. Proposition 3.13 below gives us control over the time this random walk spends in the trap $\partial^{+} A$ starting from $x \in \partial^{++} A$.

Proposition 3.13: There exist $\kappa=\kappa(A)>0$ and $\beta_{0}>0$ such that, for all $\delta>0$, all $\beta>\beta_{0}$ and all $t \in\left[e^{U \beta}, e^{C \beta}\right]$ with $U<C<\infty$,

$$
\min _{x \in \partial^{++} A} \mathbb{P}_{x}\left(\hat{\xi}_{t}^{A} \in \partial^{+} A\right) \geqslant \frac{1}{t} e^{(U-\delta) \beta} \frac{\kappa}{2(C \beta)^{2}} .
$$

Proof: Again, we first prove the analogous estimate for discrete time. The proof uses the following asymptotic result for simple random walk $\left(\xi_{n}\right)_{n \in \mathbb{N}_{0}}$ on $\mathbb{Z}^{2}$. Let $\tau_{0}=\min \left\{n>0: \xi_{n}=0\right\}$. Then there exists a $\kappa_{1}>0$ such that

$$
\mathbb{P}_{x}\left(\tau_{0}=n\right) \sim \frac{\kappa_{1}}{n \log ^{2} n} \quad\left(\forall x \in \mathbb{Z}^{2}, n \rightarrow \infty\right)
$$

(see Spitzer ${ }^{18}$ Sec. 7).

(1) From (3.15) it is easily deduced that for all rectangular $A \subset \mathbb{Z}^{2}$ there exists a $\kappa=\kappa(A)$ $>0$ such that

$$
\min _{x \in \partial^{++} A} \mathbb{P}_{x}\left(\tau_{\partial^{+} A}=n\right) \geqslant \frac{\kappa}{n \log ^{2} n} \quad(n \rightarrow \infty),
$$

where $\tau_{\partial^{+} A}=\min \left\{n>0: \xi_{n} \in \partial^{+} A\right\}$.

(2) Let $\left(\hat{\xi}_{n}^{A}\right)_{n \in \mathbb{N}_{0}}$ be the discrete-time version of (3.12). Let $n_{0}=e^{(U-\delta) \beta} \ll n$. Then, for $x$ $\in \partial^{++} A$,

$$
\mathbb{P}_{x}\left(\hat{\xi}_{n}^{A} \in \partial^{+} A\right) \geqslant(1+o(1)) \mathbb{P}_{x}\left(n-n_{0}<\tau_{\partial^{+} A} \leqslant n\right) .
$$

Here we throw away all the first hits of $\partial^{+} A$ at or prior to time $n-n_{0}$ and require the random walk to stay trapped for a time at least $n_{0}$. The latter costs not more than $\left(1-e^{-U \beta}\right)^{n_{0}}=1$ $-e^{-\delta \beta+o(\beta)}$. But, by (3.16), we have

$$
\min _{x \in \partial^{++} A} \mathbb{P}_{x}\left(n-n_{0}<\tau_{\partial^{+} A} \leqslant n\right) \geqslant \sum_{m=n-n_{0}+1}^{n} \frac{\kappa}{m \log ^{2} m} \sim \frac{\kappa n_{0}}{n \log ^{2} n} \quad(n \rightarrow \infty),
$$

and so for all $n \in\left[e^{U \beta}, e^{C \beta}\right]$ and $\beta$ sufficiently large,

$$
\min _{x \in \partial^{++} A} \mathbb{P}_{x}\left(\hat{\xi}_{n} \in \partial^{+} A\right) \geqslant \frac{1}{n} e^{(U-\delta) \beta} \frac{\kappa}{2(C \beta)^{2}} .
$$

(3) The extension to continuous time is again trivial, via a standard LD-estimate on the clock of the random walk. QED

Proposition 3.13 will be used to control the time that particles arriving from the gas stay attached to a droplet inside $\bar{\Lambda}$.

\subsection{Mixing propositions for independent random walks}

In the following propositions, $\mathcal{X}_{0}$ is the set of configurations defined in (2.26)-(2.27). 
For $\eta_{0} \in \mathcal{X}_{0}$, let $\mathbf{C}_{1}^{\gamma}\left(\eta_{0}\right)$ denote the event that no particle in $\eta_{0} \cap\left(\Lambda_{\beta} \backslash \bar{\Lambda}\right)$ enters $\bar{\Lambda}$ within time $T=e^{[\Delta-(\gamma / 2)] \beta}$ and all of them are outside $\Lambda_{l_{-}}$at time $T$. We recall that $l_{-}=e^{(1 / 2)(\Delta-\gamma) \beta}$.

Proposition 3.20: For all $\gamma>0$ there exist $\kappa>0$ and $\beta_{0}>0$ such that for all $\beta$ $>\beta_{0}: \min _{\eta_{0} \in \mathcal{X}_{0}} \mathbb{P}_{\eta_{0}}\left(\mathbf{C}_{1}^{\gamma}\left(\eta_{0}\right)\right) \geqslant(\kappa / \beta)^{\log \beta}$. have

Proof: Because outside $\bar{\Lambda}$ particles perform independent simple random walks [see (3.1)], we

$$
\min _{\eta_{0} \in \mathcal{X}_{0}} \mathbb{P}_{\eta_{0}}\left(\mathbf{C}_{1}^{\eta}\left(\eta_{0}\right)\right) \geqslant\left[\min _{x \in \partial^{+} \bar{\Lambda}} \mathbb{P}_{x}\left(\tau_{\Lambda}^{-}>T, \xi_{T} \notin \Lambda_{l_{-}}\right)\right]^{\log \beta},
$$

where we use that $N_{\Lambda_{l} \backslash \bar{\Lambda}}\left(\eta_{0}\right) \leqslant \log \beta$ for all $\eta_{0} \in \mathcal{X}_{0}$, and that the probability between square brackets is minimal in $x \in \Lambda_{l_{-}} \backslash \bar{\Lambda}$ when $x \in \partial^{+} \bar{\Lambda}$. We have

$$
\mathbb{P}_{x}\left(\tau_{\Lambda}^{-}>T, \xi_{T} \notin \Lambda_{l_{-}}\right) \geqslant \mathbb{P}_{x}\left(\tau_{\Lambda}^{-}>T\right)-\mathbb{P}_{x}\left(\xi_{T} \in \Lambda_{l_{-}}\right) .
$$

But, by Proposition 3.2, we know that

$$
\min _{x \in \partial^{+} \bar{\Lambda}} \mathbb{P}_{x}\left(\tau_{\Lambda}^{-}>T\right) \geqslant \frac{\kappa_{1}}{\log T} \sim \frac{\kappa_{1}}{\left(\Delta-\frac{\gamma}{2}\right) \beta},
$$

while (3.7) gives

$$
\max _{\partial^{+} \bar{\Lambda}} \mathbb{P}_{x}\left(\xi_{T} \in \Lambda_{l_{-}}\right) \leqslant \frac{\kappa_{2}\left|\Lambda_{l_{-}}\right|}{T}=\kappa_{2} e^{-\gamma \beta} .
$$

Insert (3.23)-(3.24) into (3.22) to get the claim. QED

For $\eta_{0} \in \mathcal{X}_{0}$, let $\mathbf{C}_{2}^{\gamma, \delta}\left(\eta_{0}\right)$ denote the event that no particle in $\eta_{0} \cap\left(\mathbb{Z}^{2} \backslash \Lambda_{l_{-}}\right)$enters $\bar{\Lambda}$ within time $T=e^{(\Delta-\delta) \beta}$.

Proposition 3.25: For all $\delta>\gamma>0: \min _{\eta_{0} \in \mathcal{X}_{0}} \mathbb{P}_{\eta_{0}}\left(\mathbf{C}_{2}^{\gamma, \delta}\left(\eta_{0}\right)\right)=1-$ SES.

Proof: We have

$$
\mathbb{P}_{\eta_{0}}\left(\mathbf{C}_{2}^{\gamma, \delta}\left(\eta_{0}\right)\right)=\prod_{x \in \mathbb{Z}^{2} \backslash \Lambda_{l_{-}}} \mathbb{P}_{x}\left(\tau_{\Lambda}^{-}>T\right)^{\eta_{0}(x)}=\prod_{x \in \mathbb{Z}^{2} \backslash \Lambda_{l_{-}}}\left[1-\mathbb{P}_{x}\left(\tau_{\Lambda}^{-} \leqslant T\right)\right]^{\eta_{0}(x)}
$$

But, by Brownian approximation, we have

$$
\mathbb{P}_{x}\left(\tau_{\Lambda}^{-} \leqslant T\right) \leqslant \exp \left[-\kappa|x|^{2} / T\right] \ll 1
$$

uniformly in $x \in \mathbb{Z}^{2} \backslash \Lambda_{l_{-}}$. Hence, for $\beta$ sufficiently large,

$$
\mathbb{P}_{\eta_{0}}\left(\mathbf{C}_{2}^{\gamma, \delta}\left(\eta_{0}\right)\right) \geqslant \exp \left\{-\frac{1}{2} \sum_{x \in \mathbb{Z}^{2} \backslash \Lambda_{l_{-}}} \eta_{0}(x) \exp \left[-\kappa|x|^{2} / T\right]\right\} .
$$

The sum in the exponent can be estimated from above by

$$
\sum_{n=0}^{\infty} N_{2^{n+1} \Lambda_{l_{-}} \mid 2^{n} \Lambda_{l_{-}}}\left(\eta_{0}\right) \exp \left[-\kappa \frac{1}{2} 2^{2 n} e^{(\delta-\gamma) \beta}\right]
$$

Hence the claim follows via (2.26). QED 
For $\eta_{0} \in \mathcal{X}_{0}, t_{1} \geqslant e^{(\Delta-2 \gamma) \beta}$ and $x_{1} \in \partial^{-} \bar{\Lambda}$, let

$$
\mathbf{C}_{3}^{\gamma}\left(\eta_{0} ; t_{1} ; x_{1}\right)
$$

denote the event that some particle from $\eta_{0} \cap\left(\mathbb{Z}^{2} \backslash \bar{\Lambda}\right)$ enters $\bar{\Lambda}$ during the time interval $\left[t_{1}, t_{1}\right.$ $+1)$ at site $x_{1}$ without having entered $\bar{\Lambda}$ during the time interval $\left[t_{1}-T_{1}, t_{1}\right)$ with $T_{1}$ $=e^{(\Delta-2 \gamma) \beta}$.

Proposition 3.31: For all $\gamma>0$ there exist $\kappa>0$ and $\beta_{0}>0$ such that for all $\beta>\beta_{0}$ :

$$
\min _{\eta_{0} \in \mathcal{X}_{0}} \mathbb{P}_{\eta_{0}}\left(\mathbf{C}_{3}^{\gamma}\left(\eta_{0} ; t_{1} ; x_{1}\right)\right) \leqslant \kappa e^{-(\Delta-2 \gamma) \beta} \log \beta
$$

uniformly in $T_{1} \leqslant t_{1} \leqslant T=e^{C \beta}$ and $x_{1} \in \partial^{-} \bar{\Lambda}$, with $C$ arbitrarily large.

Proof: Let us look at the particle configuration at time $t_{1}-T_{1}$. By Proposition 2.28 we know that with a probability $1-$ SES this configuration falls in $\mathcal{X}_{0}$. Hence, using the Markov property at time $t_{1}-T_{1}$, we get

$$
\max _{\eta_{0} \in \mathcal{X}_{0}} \mathbb{P}_{\eta_{0}}\left(\mathbf{C}_{3}^{\gamma}\left(\eta_{0} ; t_{1} ; x_{1}\right)\right)=\mathrm{SES}+\max _{\eta_{0} \in \mathcal{X}_{0}} \sum_{\eta \in \mathcal{X}_{0}} \mathbb{P}_{\eta_{0}}\left(\eta_{t_{1}-T_{1}}=\eta\right) \mathbb{P}_{\eta_{0}}\left(\mathbf{C}_{3}^{\gamma}\left(\eta_{0} ; t_{1} ; x_{1}\right) \mid \eta_{t_{1}-T_{1}}=\eta\right)
$$

But, by Proposition 3.25 and (3.7), we have

$$
\begin{aligned}
& \max _{\eta \in \mathcal{X}_{0}} \mathbb{P}_{\eta_{0}}\left(\mathbf{C}_{3}^{\gamma}\left(\eta_{0} ; t_{1} ; x_{1}\right) \mid \eta_{t_{1}-T_{1}}=\eta\right) \\
& \quad \leqslant \mathrm{SES}+\max _{\eta \in \mathcal{X}_{0}} \sum_{x \in \Lambda_{l_{-} \backslash \bar{\Lambda}}} \eta(x) \mathbb{P}_{x}\left(\xi_{T_{1}}=x_{1}\right) \\
& \quad \leqslant \mathrm{SES}+\frac{\kappa_{1}}{T_{1}} \max _{\eta \in \mathcal{X}_{0}} N_{\Lambda_{l_{-}} \backslash \bar{\Lambda}}(\eta) \leqslant \mathrm{SES}+\kappa_{1} e^{-(\Delta-2 \gamma) \beta} \log \beta .
\end{aligned}
$$

Substitution into (3.33) gives the claim. QED

Propositions 3.20, 3.25, and 3.31 will be needed to control the dynamics of the gas outside $\bar{\Lambda}$.

\section{LOCAL MARKOV CHAIN: DEFINITIONS AND SADDLE POINTS}

In this section we introduce the local Markov chain that approximates our dynamics inside $\bar{\Lambda}$, and we study its geometric properties. In Sec. 5 we will study the recurrence properties of this Markov chain, which will be needed in Secs. 6-7 to study the metastable behavior of the full Markov chain.

\subsection{Definition of the local Markov chain} define

We denote by $b=(x, y)$ an oriented bond, i.e., an ordered pair of nearest-neighbor sites, and

$$
\begin{aligned}
& \partial^{*} \bar{\Lambda}^{\text {out }}=\{b=(x, y): x \in \bar{\Lambda}, y \notin \bar{\Lambda}\}, \\
& \partial^{*} \bar{\Lambda}^{\mathrm{in}}=\{b=(x, y): x \notin \bar{\Lambda}, y \in \bar{\Lambda}\},
\end{aligned}
$$

and $\partial^{*} \bar{\Lambda}=\partial^{*} \bar{\Lambda}^{\text {out }} \cup \partial^{*} \bar{\Lambda}^{\text {in }}$. Two configurations $\bar{\eta}, \bar{\eta}^{\prime} \in \overline{\mathcal{X}}=\{0,1\}^{\bar{\Lambda}}$ with $\bar{\eta} \neq \bar{\eta}^{\prime}$ are called communicating states if there exists a bond $b \in \bar{\Lambda}^{*} \cup \partial^{*} \bar{\Lambda}$ such that $\bar{\eta}^{\prime}=T_{b} \bar{\eta}$, where $T_{b} \bar{\eta}$ is the configuration obtained from $\bar{\eta}$ as follows: 
- $b \in \bar{\Lambda}^{*}: T_{b} \bar{\eta}$ denotes the configuration obtained from $\bar{\eta}$ by interchanging particles along $b$; $\bullet b \in \partial^{*} \bar{\Lambda}^{\text {out }}$ (i.e., $b$ is exiting from $\bar{\Lambda}$ ):

$$
T_{b} \bar{\eta}(z)= \begin{cases}\bar{\eta}(z) & \forall z \neq x \\ 0 & z=x\end{cases}
$$

$\bullet b \in \partial^{*} \bar{\Lambda}^{\text {in }}$ (i.e., $b$ is entering $\bar{\Lambda}$ ):

$$
T_{b} \bar{\eta}(z)= \begin{cases}\bar{\eta}(z) & \forall z \neq y \\ 1 & z=y\end{cases}
$$

Definition 4.4: The local Markov chain $\left(\bar{\eta}_{t}\right)_{t \geqslant 0}$ is the Markov chain on $\overline{\mathcal{X}}=\{0,1\}^{\bar{\Lambda}}$ with generator

$$
(\mathcal{L} f)(\bar{\eta})=\sum_{b \in \bar{\Lambda} * \cup \partial^{*} \bar{\Lambda}} c(b, \bar{\eta})\left[f\left(T_{b} \bar{\eta}\right)-f(\bar{\eta})\right],
$$

where $\bar{H}$ is defined in (1.49) and

$$
c(b, \bar{\eta})=e^{-\beta\left[\bar{H}\left(T_{b} \bar{\eta}\right)-\bar{H}(\bar{\eta})\right]_{+}} .
$$

Note that

$$
\begin{aligned}
& b \in \partial^{*} \bar{\Lambda}^{\text {in }}: \quad c(b, \bar{\eta})=e^{-\Delta \beta}, \\
& b \in \partial^{*} \bar{\Lambda}^{\text {out: }} \quad c(b, \bar{\eta})=1 .
\end{aligned}
$$

These rates do not depend on $\bar{\eta}$ because there is no interaction between particles in $\bar{\Lambda} \backslash \bar{\Lambda}_{0}$ and particles in $\bar{\Lambda}_{0}$.

In a standard way the above dynamics can be realized with the help of Poisson clocks. To study the transitions of the local Markov chain, we consider the discrete-time version that is obtained from the continuous-time version by looking at the process when some clock in $\bar{\Lambda}^{*} \cup \partial^{*} \bar{\Lambda}$ rings. We denote by $\bar{P}\left(\bar{\eta}, \bar{\eta}^{\prime}\right)$ the corresponding transition probabilities, i.e.,

$$
\bar{P}\left(\bar{\eta}, \bar{\eta}^{\prime}\right)=\mathrm{P}_{\bar{\eta}}\left(\bar{\eta}_{\bar{\tau}_{1}}=\bar{\eta}^{\prime}\right)
$$

with $\bar{\tau}_{1}$ the first ringing time of a clock in $\bar{\Lambda}^{*} \cup \partial^{*} \bar{\Lambda}$. It is easy to verify that the stochastic dynamics defined by (4.5)-(4.6) and (4.8) is reversible w.r.t. $\bar{H}$. In particular, the transition probabilities $P\left(\bar{\eta}, \bar{\eta}^{\prime}\right)$ can be written in the form

$$
\bar{P}\left(\bar{\eta}, \bar{\eta}^{\prime}\right)=q\left(\bar{\eta}, \bar{\eta}^{\prime}\right) e^{-\beta\left[\bar{H}\left(\bar{\eta}^{\prime}\right)-\bar{H}(\bar{\eta})\right]_{+}},
$$

where $q\left(\bar{\eta}, \bar{\eta}^{\prime}\right)$ is an irreducible symmetric Markov kernel living on the set of communicating states.

\subsection{Geometric definitions}

Let us recall some definitions from Olivieri and Scoppola. ${ }^{10}$

(1) A path $\phi$ is a sequence $\phi=\phi_{1}, \ldots, \phi_{n}\left(n \in \mathbb{N}, \phi_{i} \in \overline{\mathcal{X}}\right)$ with $\bar{P}\left(\phi_{i}, \phi_{i+1}\right)>0$ for $i=1, \ldots, n$ -1 . We write $\phi: \bar{\eta} \rightarrow \bar{\eta}^{\prime}$ to denote a path from $\bar{\eta}$ to $\bar{\eta}^{\prime}$. A set $\mathcal{A} \subset \overline{\mathcal{X}}$ with $|\mathcal{A}|>1$ is connected if and only if $\forall \bar{\eta}, \bar{\eta}^{\prime} \in \mathcal{A} \quad \exists \phi: \bar{\eta} \rightarrow \bar{\eta}^{\prime}$ such that $\phi \subset \mathcal{A}$. Given $\mathcal{A} \subset \overline{\mathcal{X}}$, we define its boundary 


$$
\partial \mathcal{A}=\{\bar{\zeta} \notin \mathcal{A}: \bar{P}(\bar{\zeta}, \bar{\eta})>0\} .
$$

(2) The set of minima of the Hamiltonian $\bar{H}$ in $\mathcal{A}$ is

$$
\mathcal{F}(\mathcal{A})=\left\{\bar{\eta} \in \mathcal{A}: \bar{H}(\bar{\eta})=\min _{\bar{\zeta} \in \mathcal{A}} \bar{H}(\bar{\zeta})\right\}
$$

The communication height between $\bar{\eta}, \bar{\eta}^{\prime} \in \overline{\mathcal{X}}$ is

$$
\bar{H}\left(\bar{\eta}, \bar{\eta}^{\prime}\right)=\min _{\phi: \bar{\eta}^{\prime} \rightarrow \bar{\eta}^{\prime}} \max _{\bar{\zeta} \in \phi} \bar{H}(\bar{\zeta}) .
$$

The set of configurations realizing the minimal saddles between $\bar{\eta}, \bar{\eta}^{\prime} \in \overline{\mathcal{X}}$ is

$$
\mathcal{S}\left(\bar{\eta}, \bar{\eta}^{\prime}\right)=\left\{\bar{\zeta} \in \overline{\mathcal{X}}: \exists \phi: \bar{\eta} \rightarrow \bar{\eta}^{\prime}, \quad \phi \ni \bar{\zeta}: \max _{\bar{\xi} \in \phi} \bar{H}(\bar{\xi})=\bar{H}\left(\bar{\zeta}, \bar{\eta}^{\prime}\right)\right\} .
$$

Given two sets $\mathcal{A}, \mathcal{B} \subset \overline{\mathcal{X}}$, put

$$
\bar{H}(\mathcal{A}, \mathcal{B})=\min _{\bar{\eta} \in \mathcal{A}, \bar{\eta}^{\prime} \in \mathcal{B}} \bar{H}\left(\bar{\eta}, \bar{\eta}^{\prime}\right)
$$

and

$$
\mathcal{S}(\mathcal{A}, \mathcal{B})=\left\{\mathcal{S}\left(\bar{\eta}, \bar{\eta}^{\prime}\right): \bar{\eta} \in \mathcal{A}, \quad \bar{\eta}^{\prime} \in \mathcal{B}, \quad \bar{H}\left(\bar{\eta}, \bar{\eta}^{\prime}\right)=\bar{H}(\mathcal{A}, \mathcal{B})\right\} .
$$

(3) Next we introduce a geometric description of the configurations in terms of contours. Given a configuration $\bar{\eta} \in \overline{\mathcal{X}}$, consider the set $C(\bar{\eta}) \subset \mathrm{R}^{2}$ defined as the union of the $1 \times 1$ closed squares centered at the occupied sites of $\bar{\eta}$ in $\bar{\Lambda}_{0}$. The maximal connected components $C_{1}, \ldots, C_{m}(m \in \mathbb{N})$ of $C(\bar{\eta})$ are called clusters of $\bar{\eta}$. The centers of the unit squares of a cluster $C$ form a $*$-cluster (i.e., are within distance $\sqrt{2}$ ). The boundary of a cluster $C$ is a polygon connecting sites on the dual lattice $\left(\mathbb{Z}^{2}\right)^{*}=Z^{2}+\left(\frac{1}{2}, \frac{1}{2}\right)$. At each site an even number of bonds of this polygon meet: 0,2 or 4 . When this number is 4 , we use some convention to "round off" the corners (e.g., by connecting the northeast and the southwest sides in a unit square): in this way we obtain a further decomposition of the boundary of a cluster into a set $\bar{\gamma}_{1}, \ldots, \bar{\gamma}_{k}$ of closed self-avoiding contours (see e.g., Gallavotti ${ }^{19}$ ).

(4) Let $\bar{\eta}$ be such that $\left.\bar{\eta}\right|_{\Lambda_{0}}$ gives rise to a single contour $\bar{\gamma}=\bar{\gamma}(\bar{\eta})$. Define

$$
n(\bar{\eta})=N_{\bar{\Lambda} \backslash \bar{\Lambda}_{0}}(\bar{\eta})
$$

In this case

$$
\bar{H}(\bar{\eta})=E(\bar{\gamma})+\Delta n(\bar{\eta})
$$

where

$$
E(\bar{\gamma})=(-2 U+\Delta)\langle\bar{\gamma}\rangle+\frac{U}{2}|\bar{\gamma}|
$$

with $\langle\bar{\gamma}\rangle$ the area enclosed by $\bar{\gamma}$ and $|\bar{\gamma}|$ the perimeter of $\bar{\gamma}$. Indeed, it is easy to check that $2\langle\bar{\gamma}\rangle-\frac{1}{2}|\bar{\gamma}|$ is the number of nearest-neighbor bonds enclosed by $\bar{\gamma}$. (Note that, since there is no interaction inside $\bar{\Lambda} \backslash \bar{\Lambda}_{0}$ nor between $\bar{\Lambda}_{0}$ and $\bar{\Lambda} \backslash \bar{\Lambda}_{0}$, for the computation of $E(\bar{\gamma})$, the "energy" of $\bar{\gamma}$, everything is as if we had empty boundary conditions outside $\bar{\Lambda}_{0}$.) 


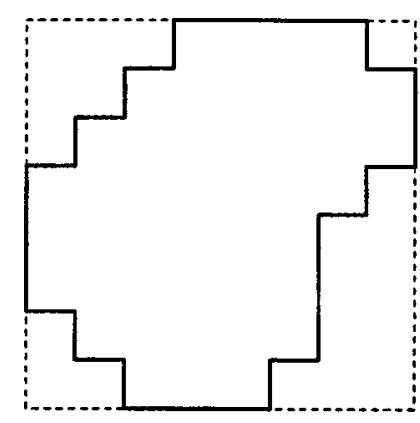

FIG. 7. A monotone contour.

(5) We denote by $R(\bar{\gamma})$ the rectangle circumscribing the contour $\bar{\gamma}$, and by $l_{1}(\bar{\gamma}), l_{2}(\bar{\gamma})$ the lengths of its sides. We use the convention $l_{1}(\bar{\gamma}) \leqslant l_{2}(\bar{\gamma})$ and collect the rectangles in equivalence classes modulo translations and rotations. We denote by $\mathcal{R}_{l_{1} l_{2}}$ the set of configurations whose single contour is an $l_{1} \times l_{2}$ rectangle. We call monotone a contour $\bar{\gamma}$ such that its perimeter coincides with that of the circumscribed rectangle: $|\bar{\gamma}|=2\left(l_{1}(\bar{\gamma})+l_{2}(\bar{\gamma})\right)$. (See Fig. 7.)

(6) Given integers $l_{1}, l_{2} \geqslant 2$ with $0 \leqslant l_{2}-l_{1} \leqslant 1$, we define:

- $\mathcal{D}_{l_{1} l_{2}}$ the set of configurations where the occupied sites form an $l_{1} \times l_{2}$ square or quasi-square contained in $\bar{\Lambda}_{0}$ plus one free particle, i.e., a particle in $\bar{\Lambda}$ not touching the rectangle.

- $\hat{\mathcal{D}}_{l_{1}, l_{2}}^{-}$the set of configurations obtained from a configuration in $\mathcal{D}_{l_{1}, l_{2}}$ by attaching the free particle to one of the sides.

- $\mathcal{D}_{l_{1}, l_{2}}^{-}$the set of configurations given by

$$
\mathcal{D}_{l_{1}, l_{2}}^{-}=\left\{\bar{\eta}^{\prime} \in \overline{\mathcal{X}}: \exists \bar{\eta} \in \hat{\mathcal{D}}_{l_{1}, l_{2}}^{-}: \bar{H}\left(\bar{\eta}, \bar{\eta}^{\prime}\right) \leqslant \bar{H}(\bar{\eta})+U, \bar{H}(\bar{\eta})=\bar{H}\left(\bar{\eta}^{\prime}\right)\right\} .
$$

In other words, $\mathcal{D}_{l_{1}, l_{2}}^{-}$is the set of configurations $\bar{\eta}^{\prime}$ that can be connected to some $\bar{\eta} \in \hat{\mathcal{D}}_{l_{1}, l_{2}}^{-}$by paths $\phi=\phi_{1}, \ldots, \phi_{n}(n \in \mathbb{N})$ such that

$$
\phi_{1}=\bar{\eta}^{\prime}, \quad \phi_{n}=\bar{\eta}, \quad \max _{1 \leqslant i<n} \bar{H}\left(\phi_{i}\right) \leqslant \bar{H}(\bar{\eta})+U, \quad \bar{H}(\bar{\eta})=\bar{H}\left(\bar{\eta}^{\prime}\right) .
$$

It is not hard to see that $\mathcal{D}_{l_{1}, l_{2}}^{-}$contains only configurations giving rise to a single monotone contour $\bar{\gamma}$ contained in $\bar{\Lambda}_{0}$ such that

$\langle\bar{\gamma}\rangle=l_{1} l_{2}+1$;

$R(\bar{\gamma})$ has side lengths $l_{1}(\bar{\gamma}) \leqslant l_{1}+1$ and $l_{2}(\bar{\gamma}) \leqslant l_{2}+1$;

$\langle\bar{\gamma}\rangle$ contains a square or quasi-square with side lengths $l_{1}-2, l_{2}-2$,

i.e., all configurations that can be obtained from $\hat{\mathcal{D}}_{l_{1}, l_{2}}^{-}$by moving particles along the border of the droplet (see Fig. 9 in Sec. 5.2).

- $\mathcal{D}_{l_{1}, l_{2}}^{0}$ the set of configurations obtained from a configuration in $\mathcal{D}_{l_{2}, l_{2}}^{-}$by adding a free particle.

- $\mathcal{D}_{l_{1}, l_{2}}^{+}$the set of configurations obtained from a configuration in $\mathcal{D}_{l_{1}, l_{2}}^{0}$ by attaching the free particle to an external corner of the contour in $\mathcal{D}_{l_{1}, l_{2}}^{-}$, i.e., an empty site with two occupied neighbors. In particular, $\mathcal{D}_{l_{1}, l_{2}}^{+}$contains the set of configurations where the occupied sites form an $l_{1} \times l_{2}$ square or quasi-square contained in $\bar{\Lambda}_{0}$ plus a $1 \times 2$ protuberance attached to one of the sides. Note that the latter set can be obtained from $\hat{\mathcal{D}}_{l_{1}, l_{2}}^{-}$by attaching a $1 \times 1$ square to the 1 $\times 1$ protuberance, in an external corner.

(7) A particularly important set of configurations, which play the role of "critical configurations," is given by 


$$
\overline{\mathcal{C}}^{*}=\mathcal{D}_{l_{c}-1, l_{c}}^{0}
$$

with

$$
l_{c}=\left\lceil\frac{U}{2 U-\Delta}\right\rceil .
$$

Recall that in the simple static analysis developed in Sec. 1.2.3 this value came out as the critical droplet size. We denote by $\Gamma=\Gamma(U, \Delta)$ the energy of the critical configuration

$$
\Gamma=\bar{H}\left(\overline{\mathcal{C}}^{*}\right)=-U\left(2 l_{c}^{2}-4 l_{c}+2\right)+\Delta\left(l_{c}^{2}-l_{c}+2\right) .
$$

We recall that $\bar{\square}$ denotes the configuration where $\bar{\Lambda}$ is empty and $\overline{\mathbf{Q}}$ the set of configurations where $\bar{\Lambda}_{0}$ is full.

\subsection{Identification of saddle points}

The following proposition is the key result of this section. It identifies the saddle points for the transitions between rectangular droplets for the local Markov chain [compare with (1.46) and Fig. 5 in Sec. 1.2].

Proposition 4.24: (i) For $2 \leqslant l<l_{c}$ :

$$
\begin{aligned}
& \mathcal{S}\left(\mathcal{R}_{l, l}, \cup_{\left(l_{1}, l_{2}\right) \neq(l, l)} \mathcal{R}_{l_{1}, l_{2}}\right)=\mathcal{S}\left(\mathcal{R}_{l, l}, \mathcal{R}_{l-1, l}\right)=\mathcal{D}_{l-1, l}^{0}, \\
& \bar{H}\left(\mathcal{D}_{l-1, l}^{0}\right)-\bar{H}\left(\mathcal{R}_{l, l}\right)=(2 U-\Delta)(l-2)+2 U, \\
& \mathcal{S}\left(\mathcal{R}_{l, l+1}, \cup_{\left(l_{1}, l_{2}\right) \neq(l, l+1)} \mathcal{R}_{l_{1}, l_{2}}\right)=\mathcal{S}\left(\mathcal{R}_{l, l+1}, \mathcal{R}_{l, l}\right)=\mathcal{D}_{l, l}^{0}, \\
& \bar{H}\left(\mathcal{D}_{l, l}^{0}\right)-\bar{H}\left(\mathcal{R}_{l, l+1}\right)=(2 U-\Delta)(l-2)+2 U .
\end{aligned}
$$

(ii) For $l \geqslant l_{c}$ :

$$
\begin{aligned}
& \mathcal{S}\left(\mathcal{R}_{l, l}, \cup_{\left(l_{1}, l_{2}\right) \neq(l, l)} \mathcal{R}_{l_{1}, l_{2}}\right)=\mathcal{S}\left(\mathcal{R}_{l, l}, \mathcal{R}_{l, l+1}\right)=\mathcal{D}_{l, l}^{0}, \\
& \bar{H}\left(\mathcal{D}_{l, l}^{0}\right)-\bar{H}\left(\mathcal{R}_{l, l}\right)=2 \Delta-U \\
& \mathcal{S}\left(\mathcal{R}_{l, l+1}, \cup_{\left(l_{1}, l_{2}\right) \neq(l, l+1)} \mathcal{R}_{l_{1}, l_{2}}\right)=\mathcal{S}\left(\mathcal{R}_{l, l+1}, \mathcal{R}_{l+1, l+1}\right)=\mathcal{D}_{l, l+1}^{0}, \\
& \bar{H}\left(\mathcal{D}_{l, l+1}^{0}\right)-\bar{H}\left(\mathcal{R}_{l, l+1}\right)=2 \Delta-U
\end{aligned}
$$

(iii)

$$
\begin{gathered}
\mathcal{S}(\bar{\square}, \overline{\mathbf{\square}})=\mathcal{D}_{l_{c}-1, l_{c}}^{0}, \\
\bar{H}(\bar{\square}, \overline{\mathbf{\square}})-\bar{H}(\bar{\square})=\Gamma .
\end{gathered}
$$

Proof: Let

$$
\mathcal{N}_{n}=\left\{\bar{\eta} \in \overline{\mathcal{X}}: N_{\bar{\Lambda}}(\bar{\eta})=\sum_{x \in \bar{\Lambda}} \bar{\eta}(x)=n\right\} .
$$

We consider the foliation of $\overline{\mathcal{X}}$ into manifolds of a constant number of particles: 


$$
\overline{\mathcal{X}}=\cup_{n=0}^{|\bar{\Lambda}|} \mathcal{N}_{n} .
$$

We will consider the sets $\mathcal{N}_{l^{2}}, \mathcal{N}_{l(l+1)}$ for $l \geqslant 2$ and investigate some of their geometric properties. Our proof will be based on the following lemma identifying certain sets of minima, minimal saddles and communication heights. After this lemma has been proved we give the proof of the proposition.

Lemma 4.30: (i) For $l \geqslant 2$ :

$$
\mathcal{F}\left(\mathcal{N}_{(l-1) l}\right)=\mathcal{R}_{l-1, l}, \quad \mathcal{F}\left(\mathcal{N}_{l^{2}}\right)=\mathcal{R}_{l, l} .
$$

(ii) For $l \geqslant 2$ :

$$
\begin{aligned}
& \mathcal{S}\left(\mathcal{R}_{l-1, l}, \mathcal{N}_{l^{2}}\right)=\mathcal{S}\left(\mathcal{N}_{(l-1) l}, \mathcal{N}_{l^{2}}\right)=\mathcal{D}_{l-1, l}^{0}, \\
& \bar{H}\left(\mathcal{R}_{l-1, l}, \mathcal{N}_{l^{2}}\right)=\bar{H}\left(\mathcal{D}_{l-1, l}^{0}\right)=\bar{H}\left(\mathcal{D}_{l-1, l}^{-}\right)+\Delta, \\
& \mathcal{S}\left(\mathcal{R}_{l, l}, \mathcal{N}_{l(l+1)}\right)=\mathcal{S}\left(\mathcal{N}_{l^{2}}, \mathcal{N}_{l(l+1)}\right)=\mathcal{D}_{l, l}^{0}, \\
& \bar{H}\left(\mathcal{R}_{l, l}, \mathcal{N}_{l(l+1)}\right)=\bar{H}\left(\mathcal{D}_{l, l}^{0}\right)=\bar{H}\left(\mathcal{D}_{l, l}^{-}\right)+\Delta .
\end{aligned}
$$

Proof: The proof uses isoperimetric inequalities.

(i) Fix $l \geqslant 2$ and consider $n=(l-1) l$ or $n=l^{2}$. Given an $\bar{\eta} \in \mathcal{N}_{n}$, the energy decreases if we translate the clusters of $\bar{\eta}$ to join them into a single cluster contained in $\bar{\Lambda}_{0}$. It further decreases if we rearrange the $1 \times 1$ squares to get a single contour $\bar{\gamma}$ (i.e., if we fill the internal "holes" with external $1 \times 1$ squares). Since under these operations the total number of particles remains fixed, to minimize the energy in $\mathcal{N}_{n}$ we just have to find the contour(s) $\bar{\gamma}$ with minimal perimeter $|\bar{\gamma}|$ among the ones with $\langle\bar{\gamma}\rangle=n$. It is clear that, starting from a contour $\bar{\gamma}^{\prime}$, by rearranging the 1 $\times 1$ squares inside we can construct a monotone contour $\bar{\gamma}$ with $R(\bar{\gamma}) \subseteq R\left(\bar{\gamma}^{\prime}\right)$ without increasing the energy. The energy associated with a monotone contour $\bar{\gamma}$ with $\langle\bar{\gamma}\rangle=n$ is

$$
E(\bar{\gamma})=(-2 U+\Delta) n+U\left(l_{1}(\bar{\gamma})+l_{2}(\bar{\gamma})\right) .
$$

To minimize $E(\bar{\gamma})$ in $\mathcal{N}_{n}$, we have to find the rectangle with minimal perimeter among those whose area is $\geqslant n$. From this the claim easily follows.

(ii) Fix $l \geqslant 2$.

(1) We first prove the claim when starting from $\mathcal{R}_{l-1, l}$. We define a set of paths $\phi: \mathcal{R}_{l-1, l}$ $\rightarrow \mathcal{N}_{l^{2}}$ as follows.

Let

$$
\phi^{\text {up }}=\phi^{\text {up }}(l-1, l): \quad \mathcal{R}_{l-1, l} \rightarrow \mathcal{D}_{l-1, l}^{+}
$$

be defined by

$$
\phi^{\mathrm{up}}=\phi_{1}, \ldots, \phi_{2}, \ldots, \phi_{3}, \ldots, \phi_{4}, \ldots, \phi_{5}
$$

with

$$
\phi_{1} \in \mathcal{R}_{l-1, l}, \quad \phi_{2} \in \mathcal{D}_{l-1, l}, \quad \phi_{3} \in \mathcal{D}_{l-1, l}^{-}, \quad \phi_{4} \in \mathcal{D}_{l-1, l}^{0}, \quad \phi_{5} \in \mathcal{D}_{l-1, l}^{+},
$$

where $\phi$ uses $\phi_{1}, \phi_{2}, \phi_{3}, \phi_{4}, \phi_{5}$ as a "skeleton" and the successive configurations in $\phi$ are obtained in the obvious way by successively adding or moving a suitable particle (see 6 in Sec. 4.2). The maximal saddle in $\phi^{\text {up }}$ is reached in $\mathcal{D}_{l-1, l}^{0}$ and is of height $\bar{H}\left(\mathcal{D}_{l-1, l}^{0}\right)=\bar{H}\left(\mathcal{D}_{l-1, l}^{-}\right)$ $+\Delta$.

Next, it is easy to see that there is a path 


$$
\phi^{\text {down }}=\phi^{\text {down }}(l-1, l): \mathcal{D}_{l-1, l}^{+} \rightarrow \mathcal{N}_{l^{2}}
$$

such that $\max _{i} \bar{H}\left(\phi_{i}^{\text {down }}, \phi_{i+1}^{\text {down }}\right)<H\left(\mathcal{D}_{l-1, l}^{-}\right)+\Delta$. Indeed, to obtain this path it suffices to successively introduce into $\bar{\Lambda}$ one new particle and fill up all the corners of the contours in $\mathcal{D}_{l-1, l}^{+}$until the arrival in $\mathcal{R}_{l, l} \subset \mathcal{N}_{l^{2}}$. Each time we add a particle, we have first an increase of energy by an amount $\Delta$, but as soon as we put this particle into a corner of the cluster in $\bar{\Lambda}_{0}$ we have a decrease of energy by an amount $2 U>\Delta$.

Thus, for each $l \geqslant 2$, the path $\left(\phi^{\text {up }}(l-1, l), \phi^{\text {down }}(l-1, l)\right)$ is a candidate to realize the mini$\max$ between $\mathcal{N}_{(l-1) l}$ and $\mathcal{N}_{l^{2}}$.

(2) When starting from $\mathcal{R}_{l, l}$ we proceed exactly in the same way to construct a path $\left(\phi^{\text {up }}(l, l), \phi^{\text {down }}(l, l)\right)$.

(3) From the proof of (i) it is immediate to see that

$$
\mathcal{F}\left(\mathcal{N}_{(l-1) l} \backslash \mathcal{R}_{l-1, l}\right)=\min _{\bar{\eta} \in \mathcal{N}_{(l-1) l} \backslash \mathcal{R}_{l-1, l}} \bar{H}(\bar{\eta})=\bar{H}\left(\mathcal{R}_{l-1, l}\right)+U
$$

From this it follows that for any path passing through $\mathcal{N}_{(l-1) l} \backslash \mathcal{R}_{l-1, l}$, once it meets $\mathcal{N}_{(l-1) l+1}$ it gets an energy $\geqslant \bar{H}\left(\mathcal{R}_{l-1, l}\right)+U+\Delta$, which is strictly larger than

$$
\max _{i=1, \ldots, 5} \bar{H}\left(\phi_{i}\right)=\bar{H}\left(\mathcal{D}_{l-1, l}^{0}\right)=\bar{H}\left(\mathcal{R}_{l-1, l}\right)+2 \Delta-U .
$$

This, in turn, implies that any path realizing the minimax between $\mathcal{N}_{(l-1) l}$ and $\mathcal{N}_{(l-1) l+1}$ has to pass through $\mathcal{R}_{l-1, l}$. Moreover, any path realizing the minimax between $\mathcal{R}_{l-1, l}$ and $\mathcal{N}_{l^{2}}$ has to enter $\mathcal{N}_{(l-1) l+1}$ through $\mathcal{D}_{l-1, l}$, which corresponds to the saddle between $\mathcal{R}_{l-1, l}$ and $\mathcal{N}_{(l-1) l+1}$. (Similarly, any path realizing the minimax between $\mathcal{N}_{l^{2}}$ and $\mathcal{N}_{l(l+1)}$ has to pass through $\mathcal{R}_{(l, l}$ and $\left.\mathcal{D}_{l, l}.\right)$

(4) At this point it is clear that paths realizing the minimax between $\mathcal{R}_{l-1, l}$ and $\mathcal{N}_{l^{2}}$ also have to pass through $\hat{\mathcal{D}}_{l-1, l}^{-}$. Indeed, any move (with a change in energy) starting from $\mathcal{D}_{l-1, l}$ and different from attaching the free particle to the rectangle would involve an energy increment of at least $U$, i.e., large enough to pass over the saddle in the path $\left(\phi^{\text {up }}(l-1, l), \phi^{\text {down }}(l-1, l)\right)$ because $U>\Delta-U$. Similarly, paths realizing the minimax between $\mathcal{N}_{l^{2}}$ and $\mathcal{N}_{l(l+1)}$ have to pass through $\hat{\mathcal{D}}_{l, l}^{-}$.

(5) Let $n=(l-1) l+1$, and consider a monotone contour $\bar{\gamma}$ with $\langle\bar{\gamma}\rangle=n$. The area of its circumscribed rectangle has to be at least $(l-1)(l+1)$, with a minimal perimeter of $4 \ell$. Similarly, for $n=l^{2}+1$ the area of a circumscribed rectangle has to be at least $l(l+1)$, with a minimal perimeter of $4 l+2$. From this it easily follows that $\mathcal{F}\left(\mathcal{N}_{(l-1) l+1}\right)$ coincides with the set of configurations containing a single monotone contour inscribed in an $l \times l$ or an $(l-1) \times(l+1)$ rectangle and containing $(l-1) l+1$ particles. Similarly, $\mathcal{F}\left(\mathcal{N}_{l^{2}+1}\right)$ coincides with the set of configurations containing a single monotone contour inscribed in an $l \times(l+1)$ rectangle and containing $l^{2}+1$ particles. In particular,

$$
\mathcal{F}\left(\mathcal{N}_{l(l-1)+1}\right) \supset \mathcal{D}_{l-1, l}^{-}, \quad \mathcal{F}\left(\mathcal{N}_{l^{2}+1}\right) \supset \mathcal{D}_{l, l}^{-}
$$

From this the claim follows. QED

We can now complete the proof of Proposition 4.24. Parts (i) and (ii) follow from Lemma 4.30. To prove part (ii), note that since every path $\phi: \bar{\square} \rightarrow \bar{\square}$ has to cross all the manifolds

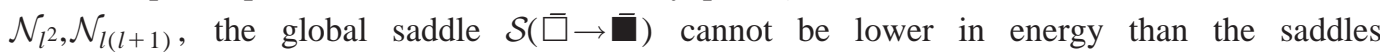
$\mathcal{S}\left(\mathcal{N}_{(l-1) l}, \mathcal{N}_{l^{2}}\right), \quad \mathcal{S}\left(\mathcal{N}_{l^{2}}, \mathcal{N}_{(l+1) l}\right), \quad l \geqslant 2$. By direct inspection we see that the saddle with maximal energy is $\mathcal{S}\left(\mathcal{N}_{\left(l_{c}-1\right) l_{c}}, \mathcal{N}_{l_{c}^{2}}\right)=\mathcal{D}_{l_{c}-1, l_{c}}^{0}$. On the other hand, using a comparison with the path $\left(\phi^{\text {up }}\left(l_{c}-1, l_{c}\right), \phi^{\text {down }}\left(l_{c}-1, l_{c}\right)\right) \quad$ we see that $\bar{H}(\mathcal{S}(\bar{\square} \rightarrow \bar{\square}))$ cannot exceed

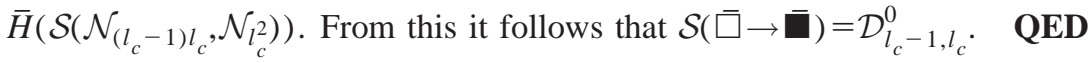


Remarks:

(1) We emphasize that, contrary to what happens under the nonconservative Glauber dynamics where the global saddle is ' $\mathcal{S}(\ominus, \oplus)=\hat{\mathcal{D}}_{l_{c}-1, l_{c}}^{-}$,' and annihilation at $\partial^{*} \bar{\Lambda}$ the global saddle is more complex and does not correspond to a single geometric shape (modulo translations and rotations). Indeed, after reaching $\hat{\mathcal{D}}_{l_{c}-1, l_{c}}^{-}$, we can, before we add a next particle whose cost is $\Delta$, perform all possible sequences of moves described by paths satisfying (4.20) and remain below the height of the global saddle, which is $\bar{H}\left(\mathcal{R}_{l_{c}-1, l_{c}}\right)+2 \Delta-U$ (see Fig. 9 in Sec. 5.2 for an example of possible moves). This global saddle is reached when we add the next particle to the configuration containing a cluster in $\mathcal{D}_{l_{c}-1, l_{c}}^{-}$[with energy $\left.\bar{H}\left(\mathcal{R}_{l_{c}-1, l_{c}}\right)+\Delta-U\right]$, giving us a configuration in $\overline{\mathcal{C}}^{*}=\mathcal{D}_{l_{c}-1, l_{c}}^{0}$. This is the set that appears in Theorem 1.53 and that plays the role of the set of critical configurations.

(2) A typical $\bar{\eta} \in \mathcal{D}_{l_{1}, l_{2}}^{-}$can in fact be quite asymmetric, i.e., quite different from square or quasi-square. However, there is always a path from $\bar{\eta}$ to a suitable square or quasi-square along which the energy does not exceed $\bar{H}(\bar{\eta})$. Thus, under the Kawasaki dynamics the squares or quasi-squares act as attractors on a time scale $e^{\Delta \beta}$, which is much shorter than the time needed to grow or shrink.

(3) The specification that we included in the geometric characterization of $\mathcal{D}_{l_{1}, l_{2}}^{-}, \mathcal{D}_{l_{1}, l_{2}}^{0}$, and $\mathcal{D}_{l_{1}, l_{2}}^{+}$is related to the fact that the above-mentioned moves can only be performed on the external boundary of the rectangles circumscribing the clusters in $\hat{\mathcal{D}}_{l_{1}, l_{2}}^{-}$.

\section{LOCAL MARKOV CHAIN: RECURRENCE}

In this section we analyze the local Markov chain $\left(\bar{\eta}_{t}\right)_{t \geqslant 0}$ on $\bar{\Lambda}$ that was defined in Sec. 4.1. Since this Markov chain is finite, it falls in the Freidlin-Wentzel regime [recall (1.19)] and the analysis of metastability can in principle be carried out by using the general method in Olivieri and Scoppola. ${ }^{10}$ The result obtained in Proposition 4.24 in Sec. 4.3, i.e., the solution of a certain sequence of minimax problems, is the "model dependent" part of this method.

It is more convenient to use the renormalization procedure developed in Scoppola, ${ }^{20}$ i.e., an analysis on suitably separated time scales, since it can be extended to the full Markov chain $\left(\eta_{t}\right)_{t \geqslant 0}$. This procedure is based on the following idea:

(1) Group the configurations in $\overline{\mathcal{X}}$ into a sequence of subsets of configurations of increasing regularity:É $\overline{\mathcal{X}} \supset \overline{\mathcal{X}}_{1} \supset \overline{\mathcal{X}}_{2} \supset \overline{\mathcal{X}}_{3} \supset \cdots$.

(2) Prove a recurrence property of the Markov chain to these sets on an increasing sequence of time scales: $T_{1} \ll T_{2} \ll T_{3} \ll \cdots$.

(3) Construct a sequence of Markov chains by observing the original Markov chain when it enters these sets, and estimate the corresponding transition probabilities.

Actually, we will not follow the renormalization procedure in full detail. Rather, we will make a construction that is adapted to our specific situation. In our case we need three sets: $\overline{\mathcal{X}}_{1} \supset \overline{\mathcal{X}}_{2} \supset \overline{\mathcal{X}}_{3}$. In Sec. 5.1 we define these sets, in Sec. 5.2 we give a geometric description of their configurations, and in Sec. 5.3 we prove the recurrence properties to these sets on the time scales $T_{1}=e^{0 \beta}, T_{2}=e^{U \beta}, T_{3}=e^{\Delta \beta}$. Section 5.4 contains some results on so-called cycles.

The results obtained in this section will be extended to the full Markov chain $\left(\eta_{t}\right)_{t \geqslant 0}$ in Secs. 6-7, and will be used in Sec. 8 to prove our main result in Theorem 1.53.

\subsection{Definition of the recurrence sets}

We begin by defining a notion of reduction of a configuration that will be needed to control the dynamics.

Definition 5.1: A configuration $\bar{\eta} \in \overline{\mathcal{X}}$ is 0-REDUCIBLE if there exists a sequence of bonds $b_{1}, \ldots, b_{k} \in \bar{\Lambda}^{*} \cup \partial^{*} \bar{\Lambda}(k \in \mathbb{N})$ such that: 
(a) $\bar{H}\left(\bar{\eta}_{i+1}\right) \leqslant \bar{H}\left(\bar{\eta}_{i}\right)$ for all $0 \leqslant i<k$,

(b) $\bar{H}\left(\bar{\eta}_{k}\right)<\bar{H}(\bar{\eta})$,

where $\bar{\eta}_{i}=T_{b_{i}} T_{b_{i-1}} \cdots T_{b_{1}} \bar{\eta}, i \geqslant 0$.

Remarks:

(1) We can always extract a subsequence of bonds with length $k \leqslant t_{0}=\left|\bar{\Lambda}^{*} \cup \partial^{*} \bar{\Lambda}\right|^{2}$, again satisfying (a) and (b), such that $\bar{\eta}_{i} \neq \bar{\eta}_{j}$ for all $i \neq j$. This is because $t_{0}$ is an upper bound for the maximal number of moves inside $\bar{\Lambda}$ needed to change any $\bar{\eta} \in \overline{\mathcal{X}}$ into any $\bar{\eta}^{\prime} \in \overline{\mathcal{X}}$.

(2) By definition, if $\bar{\eta}$ is 0 -reducible, then there exists a finite path $\phi: \bar{\eta} \rightarrow \bar{\eta}^{\prime}$ with $\bar{\eta}^{\prime}$ being a configuration that is not 0 -reducible such that $\bar{H}\left(\phi_{i+1}\right) \leqslant \bar{H}\left(\phi_{i}\right)$ for all $i$. In fact, to construct such a path it suffices to glue together the paths given by the definition of 0 -reducible configurations until we arrive at a configuration that is not 0 -reducible. The number of 0 -reductions necessary to arrive at a configuration that is not 0-reducible is finite, because with each 0-reduction the energy $\bar{H}$ decreases by at least $U$ while $\bar{H}$ is bounded from below.

Definition 5.2: The configurations $\bar{\eta}, \bar{\eta}^{\prime}$ are 0-EQUIVALENT if there exists a sequence of bonds $b_{1}, \ldots, b_{k} \in \bar{\Lambda} * \cup \partial^{*} \bar{\Lambda}(k \in \mathbb{N})$ such that $\bar{H}\left(\bar{\eta}_{i+1}\right)=\bar{H}\left(\bar{\eta}_{i}\right)$ for all $0 \leqslant i<k$ and $\bar{\eta}_{k}=\bar{\eta}^{\prime}$.

Definition 5.3: A set of configurations $\mathcal{C} \subset \overline{\mathcal{X}}$ is a CYCLE if it is connected and satisfies

$$
\bar{H}(\mathcal{F}(\partial \mathcal{C}))>\max _{\bar{\eta} \in \mathcal{C}} \bar{H}(\bar{\eta}),
$$

where $\mathcal{F}(\partial \mathcal{C})$ is the set of minima of $\bar{H}$ in the boundary $\partial \mathcal{C}$ of $\mathcal{C}$ [recall (4.10)-(4.11)].

Next we generalize the idea of reduction as follows.

Definition 5.5: A V-PATH is a finite connected sequence $\phi_{1}, \ldots, \phi_{k}(k \in \mathbb{N})$ of configurations or sets (!) of configurations such that:

(a) If $\phi_{i}=\mathcal{C}$ for some $1<i<k$ is a set of configurations, then $\mathcal{C}$ is a cycle with $\bar{H}(\mathcal{F}(\partial \mathcal{C}))$ $-\bar{H}(\mathcal{F}(\mathcal{C})) \leqslant V$, where $\mathcal{F}(\mathcal{C})$ is the set of minima of $\bar{H}$ in $\mathcal{C}$, and $\phi_{i+1}, \phi_{i-1}$ are single configurations such that $\phi_{i+1} \in \mathcal{F}(\partial \mathcal{C})$ and $\phi_{i-1} \in \mathcal{C}$.

(b) If $\phi_{i}, \phi_{i+1}$ for some $1 \leqslant i<k$ are single configurations, then $q\left(\phi_{i}, \phi_{i+1}\right)>0$ and $\bar{H}\left(\phi_{i+1}\right) \leqslant \bar{H}\left(\phi_{i}\right)$ [recall (4.9)].

The reader should think of a $V$-path as a "downhill cascade" in which a sequence of "lakes" of depth at most $V$ can be present.

Definition 5.6: A configuration $\bar{\eta}^{\prime}$ is V-REACHABLE from $\bar{\eta}$ if there exists a V-path from $\bar{\eta}$ to $\bar{\eta}^{\prime}$. Two configurations $\bar{\eta}$ and $\bar{\eta}^{\prime}$ are $V$-EQUIVALENT if $\bar{\eta}$ is $V$-reachable from $\bar{\eta}^{\prime}$ and vice versa.

Definition 5.7: A configuration $\bar{\eta}$ is V-REDUCIBLE if there exists a configuration $\bar{\eta}^{\prime}$ that is $V$-reachable from $\bar{\eta}$ such that $H\left(\bar{\eta}^{\prime}\right)<\bar{H}(\bar{\eta})$.

With these notions we define the following sets:

$$
\begin{aligned}
& \overline{\mathcal{X}}_{1}=\{\bar{\eta} \in \overline{\mathcal{X}}: \quad \bar{\eta} \text { is not } 0 \text {-reducible }\}, \\
& \overline{\mathcal{X}}_{2}=\{\bar{\eta} \in \overline{\mathcal{X}}: \quad \bar{\eta} \text { is not } U \text {-reducible }\}, \\
& \overline{\mathcal{X}}_{3}=\{\bar{\eta} \in \overline{\mathcal{X}}: \quad \bar{\eta} \text { is not } \Delta \text {-reducible }\} .
\end{aligned}
$$

We note that if $V<V^{\prime}$, then a configuration that is $V$-reducible is also $V^{\prime}$-reducible, so $\overline{\mathcal{X}}_{3} \subset \overline{\mathcal{X}}_{2} \subset \overline{\mathcal{X}}_{1}$. We also define the following sets: 


$$
\begin{aligned}
& \mathcal{E}_{1}(\bar{\eta})=\left\{\bar{\eta}^{\prime} \in \overline{\mathcal{X}}_{1}: \bar{\eta}^{\prime} \text { is 0-equivalent to } \bar{\eta}\right\} \quad\left(\bar{\eta} \in \overline{\mathcal{X}}_{1}\right), \\
& \mathcal{E}_{2}(\bar{\eta})=\left\{\bar{\eta} \in \overline{\mathcal{X}}_{2}: \bar{\eta}^{\prime} \quad \text { is } U \text {-equivalent to } \bar{\eta}\right\} \quad\left(\bar{\eta} \in \overline{\mathcal{X}}_{2}\right), \\
& \mathcal{E}_{3}(\bar{\eta})=\left\{\bar{\eta} \in \overline{\mathcal{X}}_{3}: \bar{\eta}^{\prime} \quad \text { is } \Delta \text {-equivalent to } \bar{\eta}\right\} \quad\left(\bar{\eta} \in \overline{\mathcal{X}}_{3}\right) .
\end{aligned}
$$

\subsection{Geometric description of the recurrence sets}

Next we introduce some geometric objects that will be needed to characterize the $\overline{\mathcal{X}}_{i}$ 's.

Definition 5.10:

(a) For $x \in \bar{\Lambda}_{0}$, let $n n(x)=\left\{y \in \bar{\Lambda}_{0}:|x-y|=1\right\}$ be the set of nearest-neighbor sites of $x$ in $\bar{\Lambda}_{0}$.

(b) A FREE PARTICLE is a site $x \in \bar{\eta} \cap \bar{\Lambda}_{0}$ such that $\Sigma_{y \in n n(x)} \bar{\eta}(y)=0$.

(c) A PROTUBERANCE is a site $x \in \bar{\eta} \cap \bar{\Lambda}_{0}$ such that $\Sigma_{y \in n n(x)} \bar{\eta}(y)=1$.

(d) For $\bar{\eta} \in \overline{\mathcal{X}}$, the EXTERNAL BOUNDARY $\partial \bar{\eta}$ is the set of occupied sites in $\bar{\eta}$ that can be connected to the ring $\bar{\Lambda} \backslash \bar{\Lambda}_{0}$ via a path along unoccupied sites in $\bar{\eta}$.

(e) For $\bar{\eta} \in \overline{\mathcal{X}}$, an EXTERNAL CORNER is a site $x \notin \bar{\eta}$ such that there exist $y, y^{\prime}$ $\in \partial \bar{\eta} \cap n n(x), y \neq y^{\prime}$ (see Fig. 8).

(f) $R\left(l_{h}, l_{v}, d_{n}, d_{e}, d_{s}, d_{w}\right)$ denotes the RECTANGLE in $\bar{\Lambda}_{0}$ of horizontal side length $l_{h}$ and vertical side length $l_{v}$, such that its north side is a distance $d_{n}$ from the north side of $\bar{\Lambda}$, and so on.

(g) $\quad R_{l_{1}, l_{2}}$ denotes a rectangle of side lengths $l_{1}, l_{2}$ anywhere in the box $\bar{\Lambda}$, including all translations and rotations (with the convention $l_{1} \leqslant l_{2}$ ). A rectangle $R_{l_{1}, l_{2}}$ is called a SQUARE or $a$ QUASI-SQUARE if $l_{1} \geqslant 2$ and $0 \leqslant l_{2}-l_{1} \leqslant 1$.

(h) $\mathcal{L}$ denotes the set of LACUNARY SQUARES or LACUNARY QUASI-SQUARES i.e., configurations with an external boundary given by a square or quasi-square and with holes such that the configuration is not U-reducible.

The following proposition is our main structural identification of the sets $\overline{\mathcal{X}}_{1}, \overline{\mathcal{X}}_{2}, \overline{\mathcal{X}}_{3}$.

Proposition 5.11:

(i) Configurations in $\overline{\mathcal{X}}_{1}$ have no free particles nor free holes. If $\bar{\eta}$ is connected with a monotone contour, then $\bar{\eta} \in \overline{\mathcal{X}}_{1}$.

(ii) Configurations in $\overline{\mathcal{X}}_{2}$ have no protuberances. If $\bar{\eta}$ is connected with a monotone contour obtained from a square or a quasi-square by removing $m$ sites with $0 \leqslant m \leqslant l_{1}-2$, then $\bar{\eta} \in \overline{\mathcal{X}}_{2}$.

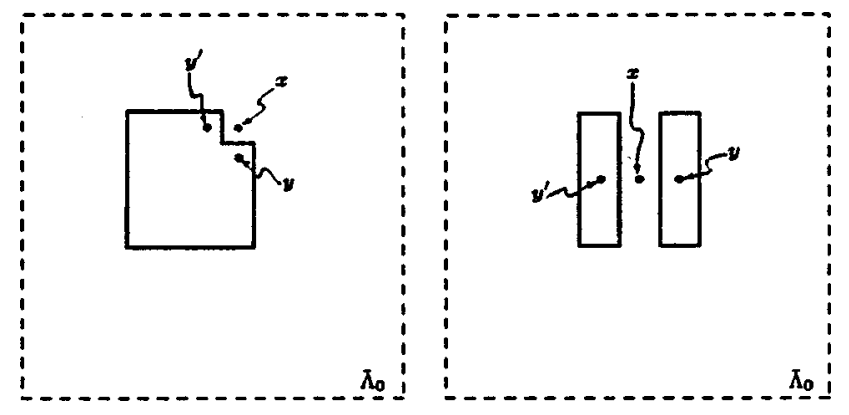

FIG. 8. Two examples of external corners. 
(iii) Configurations in $\overline{\mathcal{X}}_{3}$ can be characterized as follows: $\bar{\square} \in \overline{\mathcal{X}}_{3}$; if $\bar{\eta} \in \overline{\mathcal{X}}_{3} \backslash\{\bar{\square}\}$, then either $\bar{\eta}$ is a square or quasi-square, or $\bar{\eta}$ is a lacunary square or quasi-square with side length $>l_{0}$ /3. Moreover, if $\max \left\{d_{n}, d_{s}\right\}>l_{v}$ and $\max \left\{d_{e}, d_{w}\right\}>l_{h}$, then $\bar{\eta}=R\left(l_{h}, l_{v}, d_{n}, d_{e}, d_{s}, d_{w}\right)$.

\section{Remarks:}

(1) The characterization of $\overline{\mathcal{X}}_{1}, \overline{\mathcal{X}}_{2}$ in Proposition 5.11 (i)-(ii) is not complete. Only those properties are given that are needed to derive the complete characterization of $\overline{\mathcal{X}}_{3}$ in Proposition 5.11 (iii).

(2) The configurations in $\mathcal{L}$ need a separate treatment (see the end of Sec. 7.7).

(3) It follows from the proof of Proposition 5.11 (iii) given below that if $\mathcal{E}_{3}(\bar{\eta})$ corresponds to a "small" cluster, i.e., a cluster of side length $\leqslant l_{0} / 3$, then any $\bar{\eta}^{\prime} \in \mathcal{E}_{3}(\bar{\eta})$ can be obtained from $\bar{\eta}$ by means of a rigid motion. For this reason we will denote the elements in $\mathcal{E}_{3}(\bar{\eta}) \cap \overline{\mathcal{X}}_{3}$ corresponding to "small" clusters by $R_{l_{1}, l_{2}}$ (with $l_{1} \geqslant 2$ and $0 \leqslant l_{2}-l_{1} \leqslant 1$ ).

To prove Proposition 5.11 we need the following lemma, which will also serve us later on. For $\bar{\eta} \in \overline{\mathcal{X}}$ and $V \in\{U, \Delta, 2 U\}$, define

$$
\overline{\mathcal{C}}_{\bar{\eta}}^{V}=\left\{\bar{\eta}^{\prime} \in \overline{\mathcal{X}}: \bar{H}\left(\bar{\eta}, \bar{\eta}^{\prime}\right)-\bar{H}(\bar{\eta})<V\right\} .
$$

The structure of this set is characterized as follows.

Lemma 5.13:

(i) If $\bar{\eta} \in \overline{\mathcal{X}}_{1}$, then $\overline{\mathcal{C}}_{\bar{\eta}}^{U}$ is a cycle, $\bar{\eta} \in \mathcal{F}\left(\overline{\mathcal{C}}_{\bar{\eta}}^{U}\right)$ and $\overline{\mathcal{C}}_{\bar{\eta}}^{U} \cap \overline{\mathcal{X}}_{1}=\mathcal{E}_{1}(\bar{\eta})$.

(ii) If $\bar{\eta} \in \overline{\mathcal{X}}_{2}$, then $\overline{\mathcal{C}}_{\bar{\eta}}^{\Delta}$ is a cycle, $\bar{\eta} \in \mathcal{F}\left(\overline{\mathcal{C}}_{\bar{\eta}}^{\Delta}\right)$ and $\overline{\mathcal{C}}_{\bar{\eta}}^{\Delta} \cap \overline{\mathcal{X}}_{2}=\mathcal{E}_{2}(\bar{\eta})$. Moreover, $\overline{\mathcal{C}}_{\bar{\eta}}^{\Delta} \backslash \mathcal{F}\left(\overline{\mathcal{C}}_{\bar{\eta}}^{\Delta}\right) \subset \overline{\mathcal{X}} \backslash \overline{\mathcal{X}}_{1}$.

(iii) If $\bar{\eta} \in \overline{\mathcal{X}}_{3}$, then $\overline{\mathcal{C}}_{\bar{\eta}}^{2 U}$ is a cycle, $\bar{\eta} \in \mathcal{F}\left(\overline{\mathcal{C}}_{\bar{\eta}}^{2 U}\right)$ and $\overline{\mathcal{C}}_{\bar{\eta}}^{2 U} \cap \overline{\mathcal{X}}_{3}=\mathcal{E}_{3}(\bar{\eta})$. Moreover, $\overline{\mathcal{C}}_{\bar{\eta}}^{2 U} \backslash \mathcal{F}\left(\mathcal{C}_{\bar{\eta}}^{2 U}\right) \subset \overline{\mathcal{X}} \overline{\mathcal{X}}_{2}$

Proof: (i) If $\bar{\eta} \in \overline{\mathcal{X}}_{1}$, then $\bar{\eta} \in \mathcal{F}\left(\overline{\mathcal{C}}_{\bar{\eta}}^{U}\right)$. Indeed, if there exists $\bar{\eta}^{\prime} \in \overline{\mathcal{C}}_{\bar{\eta}}^{U}$ with $\bar{H}\left(\bar{\eta}^{\prime}\right)<\bar{H}(\bar{\eta})$, then $\bar{\eta}$ is 0-reducible, which contradicts $\bar{\eta} \in \overline{\mathcal{X}}_{1}$. Next, let $\overline{\mathcal{D}}_{\bar{\eta}}^{U}$ be the maximal connected component containing $\bar{\eta}$ of configurations $\bar{\eta}^{\prime}$ such that $\bar{H}\left(\bar{\eta}^{\prime}\right)<\bar{H}(\bar{\eta})+U$. By definition, $\overline{\mathcal{D}}_{\bar{\eta}}^{U}$ is a cycle. It turns out that $\overline{\mathcal{C}}_{\bar{\eta}}^{U}=\overline{\mathcal{D}}_{\bar{\eta}}^{U}$. Indeed, if $\bar{\eta}^{\prime} \in \overline{\mathcal{C}}_{\bar{\eta}}^{U}$, then $\bar{H}\left(\bar{\eta}^{\prime}\right) \leqslant \bar{H}\left(\bar{\eta}^{\prime}, \bar{\eta}\right)$, so $\bar{\eta}^{\prime}$ is in the connected component because the trajectory realizing the minimax gives the connection. Conversely, if $\bar{\eta}^{\prime}$ $\in \overline{\mathcal{D}}_{\bar{\eta}}^{U}$, then there exists a path $\phi: \bar{\eta} \rightarrow \bar{\eta}^{\prime}$ such that $\max _{i} \bar{H}\left(\phi_{i}\right)-\bar{H}(\bar{\eta})<U$, while by the minimax definition we have $\bar{H}\left(\bar{\eta}, \bar{\eta}^{\prime}\right) \leqslant \max _{i} \bar{H}\left(\phi_{i}\right)$. Hence $\bar{H}\left(\bar{\eta}, \bar{\eta}^{\prime}\right)-\bar{H}(\bar{\eta})<U$. Finally, if $\bar{\eta}^{\prime}$ $\in \overline{\mathcal{C}}_{\bar{\eta}}^{U} \cap \overline{\mathcal{X}}_{1}$, then it is straightforward to show that $\bar{\eta}^{\prime} \in \mathcal{F}\left(\overline{\mathcal{C}}_{\bar{\eta}}^{U}\right)$, via the same argument that was used to show that $\bar{\eta} \in \mathcal{F}\left(\overline{\mathcal{C}}_{\bar{\eta}}^{U}\right)$. This implies $\bar{H}(\bar{\eta})=\bar{H}\left(\bar{\eta}^{\prime}\right)$ and $\bar{\eta}^{\prime} \in \mathcal{E}_{1}(\bar{\eta})$.

(ii)-(iii) The proof of the first part of (ii) and (iii) can be done in the same way. The second part of (ii) follows from the following remark. If $\bar{\eta}^{\prime} \in \overline{\mathcal{X}}_{1}$, then $\bar{H}\left(\bar{\eta}^{\prime}, \bar{\eta}^{\prime \prime}\right) \geqslant \bar{H}\left(\bar{\eta}^{\prime}\right)+U$ for all $\bar{\eta}^{\prime \prime}$ with $\bar{H}\left(\bar{\eta}^{\prime \prime}\right)<\bar{H}\left(\bar{\eta}^{\prime}\right)$. On the other hand, if $\bar{\eta}^{\prime} \notin \mathcal{F}\left(\overline{\mathcal{C}}_{\bar{\eta}}^{\Delta}\right)$, then $\bar{H}\left(\bar{\eta}^{\prime}\right)=\bar{H}(\bar{\eta})+U$. But then $\bar{H}\left(\bar{\eta}^{\prime}, \bar{\eta}^{\prime \prime}\right) \geqslant \bar{H}\left(\bar{\eta}^{\prime}\right)+U=\bar{H}(\bar{\eta})+2 U$, and so $\bar{\eta}^{\prime} \notin \overline{\mathcal{C}}_{\bar{\eta}}^{\Delta}$. A similar argument works for the second part of (iii). QED

Proof of Proposition 5.11. (i) If $\bar{\eta}$ has a free particle or a free hole, then $\bar{\eta}$ is obviously 0-reducible, i.e., $\bar{\eta} \notin \overline{\mathcal{X}}_{1}$. If $\bar{\eta}$ is a connected configuration with a monotone contour, then there exist other configurations that are 0-equivalent to $\bar{\eta}$ only if $\bar{\eta}$ has at least one protuberance. In this case all the configurations that are 0 -equivalent to $\bar{\eta}$ can be obtained from $\bar{\eta}$ by moving the 
protuberance along the side of the cluster. Therefore $\bar{H}\left(T_{b} \bar{\eta}\right)-\bar{H}(\bar{\eta}) \geqslant U$ for all $b \in \bar{\Lambda}^{*} \cup \partial^{*} \bar{\Lambda}$ such that $T_{b} \bar{\eta} \notin \mathcal{E}_{1}(\bar{\eta})$.

(ii) We divide the proof into three steps.

(1) If $\bar{\eta}$ has a protuberance, then it is obvious that $\bar{\eta}$ is $U$-reducible. To prove the second claim, we first show that if $\bar{\eta}$ is a connected configuration with a monotone contour obtained by removing $0 \leqslant m \leqslant l_{1}-2$ particles from $R_{l_{1}, l_{2}}$, then to reach a configuration $\bar{\eta}^{\prime}$ with $\bar{H}\left(\bar{\eta}^{\prime}\right)$ $<\bar{H}(\bar{\eta})$ we have to pass over a saddle

$$
\bar{H}\left(\bar{\eta}, \bar{\eta}^{\prime}\right) \geqslant \bar{H}(\bar{\eta})+\Delta .
$$

The second claim follows from this inequality. Indeed, we only have to note that if there exists a $U$-path $\phi: \bar{\eta} \rightarrow \bar{\eta}^{\prime}$, then $\bar{H}\left(\bar{\eta}, \bar{\eta}^{\prime}\right) \leqslant \max _{i} \max _{\zeta \in \phi_{i}} \bar{H}(\zeta) \leqslant \bar{H}(\bar{\eta})+U$, which contradicts (5.14). Hence $\bar{\eta} \in \overline{\mathcal{X}}_{2}$.

(2) Equation (5.14) says that [recall (5.12)]

$$
\bar{\eta} \in \mathcal{F}\left(\overline{\mathcal{C}}_{\bar{\eta}}^{\Delta}\right) .
$$

Let us use the description of the energy $\bar{H}$ in terms of contours that was introduced in Sec. 4.2. For $\bar{\eta}^{\prime} \in \bar{\Lambda}$ with contour $\bar{\gamma}^{\prime}=\bar{\gamma}^{\prime}\left(\bar{\eta}^{\prime}\right)$ we have

$$
\bar{H}\left(\bar{\eta}^{\prime}\right)=(-2 U+\Delta) n_{0}^{\prime}\left(\bar{\gamma}^{\prime}\right)+\frac{U}{2}\left|\bar{\gamma}^{\prime}\right|+n^{\prime}\left(\bar{\gamma}^{\prime}\right) \Delta,
$$

where $n_{0}^{\prime}=n_{0}^{\prime}\left(\bar{\gamma}^{\prime}\right)$ is the number of particles in $\bar{\Lambda}_{0}$ (i.e., the area inside the contour) and $n^{\prime}$ $=n^{\prime}\left(\bar{\gamma}^{\prime}\right)$ is the number of particles in the ring $\bar{\Lambda} \backslash \bar{\Lambda}_{0}$. Our configuration $\bar{\eta}$ has $l_{1} l_{2}-l_{1}+2 \leqslant n_{0}$ $\leqslant l_{1} l_{2},|\bar{\gamma}|=2\left(l_{1}+l_{2}\right)$ and $n=0$.

(3) Denote by $\operatorname{CR}\left(n_{0}\right)$ the set of all configurations $\bar{\eta}^{\prime}$ such that $n_{0}^{\prime}=n_{0}$ and such that the circumscribed rectangle is contained in the square or quasi-square $R_{l_{1}, l_{2}}$. To prove (5.15) we first note that

$$
\bar{\eta} \in \mathcal{F}\left(\overline{\mathcal{C}}_{\bar{\eta}}^{\Delta} \cap \mathrm{CR}\left(n_{0}\right)\right) .
$$

Indeed, suppose that there exists a configuration $\bar{\eta}^{\prime} \in \overline{\mathcal{C}}_{\bar{\eta}}^{\Delta} \cap \mathrm{CR}\left(n_{0}\right)$ with $\left|\bar{\gamma}^{\prime}\right|<|\bar{\gamma}|$. Let $R_{l_{1}^{\prime}, l_{2}^{\prime}}^{\prime}$ be the rectangle circumscribing $\bar{\eta}^{\prime}$. Then $R_{l_{1}^{\prime}, l_{2}^{\prime}}^{\prime} \varsubsetneqq R_{l_{1}, l_{2}}$, and we have

$$
l_{1}^{\prime} \leqslant l_{1}, \quad l_{2}^{\prime} \leqslant l_{2}, \quad l_{1}^{\prime}+l_{2}^{\prime}<l_{1}+l_{2}, \quad l_{1}^{\prime} l_{2}^{\prime} \geqslant n_{0}=l_{1} l_{2}-m
$$

with $0 \leqslant m \leqslant l_{1}-2$. But $l_{1}^{\prime}<l_{1}$ implies $l_{1}^{\prime} l_{2}^{\prime} \leqslant\left(l_{1}-1\right) l_{2}<n_{0}$ and $l_{2}^{\prime}<l_{2}$ implies $l_{1}^{\prime} l_{2}^{\prime} \leqslant l_{1}\left(l_{2}-1\right)$ $<n_{0}$, which contradicts (5.18). The final step in the proof is to show that

$$
\overline{\mathcal{C}}_{\bar{\eta}}^{\Delta} \cap \mathrm{CR}\left(n_{0}\right)=\overline{\mathcal{C}}_{\bar{\eta}}^{\Delta} .
$$

But this is an immediate consequence of the fact that in order to exit from $\operatorname{CR}\left(n_{0}\right)$ the process has to reach an energy $\geqslant \bar{H}(\bar{\eta})+\Delta$.

(iii) We divide the proof into five steps. Namely, for every $\bar{\eta} \in \overline{\mathcal{X}}_{3}$ we show that

(1) $\partial \bar{\eta}$ is the boundary of a union of rectangles;

(2) these rectangles are squares or quasi-squares;

(3) squares or quasi-squares can move;

(4) $\partial \bar{\eta}$ is the boundary of a single square or quasi-square;

(5) a "small" square or quasi-square is not lacunary. 
(1) Suppose that $\bar{\eta}$ has an external corner $x$. Then $\bar{\eta}$ is $\Delta$-reducible, because a particle can be created at cost $\Delta$ at $\partial^{-} \bar{\Lambda}$ and can be moved inside the external corner at $x$, which gives us a $\Delta$-path (recall from Lemma 5.13 that $\overline{\mathcal{C}}_{\bar{\eta}}^{\Delta}$ is a cycle). The only case in which $\partial \bar{\eta}$ has no external corners is when $\partial \bar{\eta}$ is the boundary of a union of rectangles at distances $>2$ (recall Fig. 8).

(2) Suppose that $\bar{\eta}=R\left(l_{h}, l_{v}, d_{n}, d_{e}, d_{s}, d_{w}\right)$ with $l_{h}-l_{v}>1$. We want to show that $\bar{\eta} \notin \overline{\mathcal{X}}_{3}$. Also now we can find a $\Delta$-reduction of $\bar{\eta}$ (recall Definition 5.5: to each of the pictures in Fig. 9 in fact associate the full corresponding $U$-cycle, which is not drawn). This type of movement is studied in more detail in Peixoto. ${ }^{21}$

(3) By using the same kind of path as in Fig. 9, we can move the square or quasi-square around. The $\Delta$-path achieving an upward movement is shown in Fig. 10.

(4) Since, by (3), squares and quasi-squares can move around, any configuration with more than one square or quasi-square can be $\Delta$-reduced by moving the pieces together.

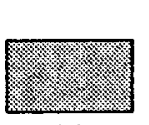

(1)

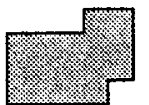

(6)

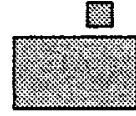

(2)

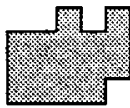

(7)

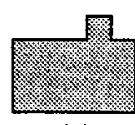

(3)

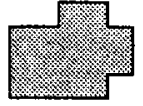

(8)

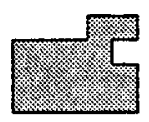

(4)

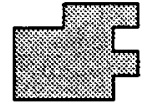

(9)

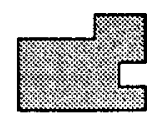

(5)

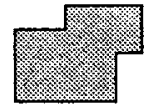

(10)

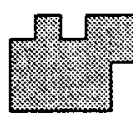

(11)

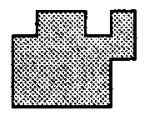

(12)

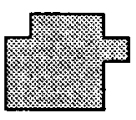

(13)

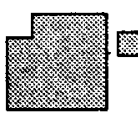

(14)

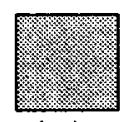

(15)

FIG. 9. Movement of particles along the border: $5 \times 3+1=4 \times 4$.

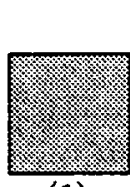

(1)

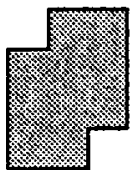

(6)

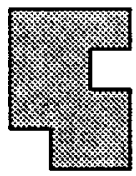

(11)

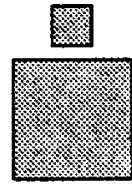

(2)

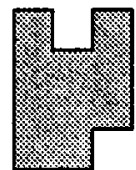

(7)

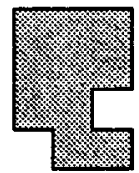

(12)

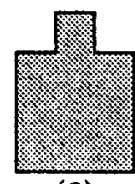

(3)

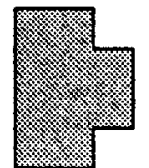

(8)

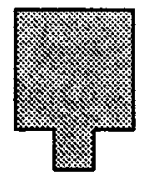

(13)

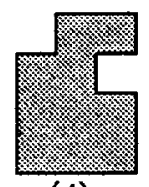

(4)

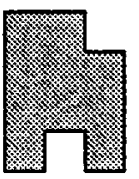

(9)

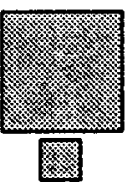

(14)

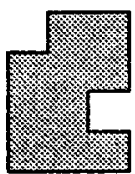

(5)

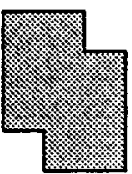

(10)

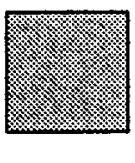

(15)

FIG. 10. Upward movement of a $3 \times 3$ square. 
(5) Again by (3), for lacunary squares or quasi-squares with $\max \left\{d_{n}, d_{s}\right\}>l_{v}$ and $\max \left\{d_{e}, d_{w}\right\}$ $>l_{h}$ there is sufficient room to move them up so as to create an opening for the internal structure. Indeed, Fig. 10 shows a motion of particles along the boundary where the holes in the cluster do not move. QED

\subsection{Recurrence}

Let $T_{1}=e^{0 \beta}, T_{2}=e^{U \beta}, T_{3}=e^{\Delta \beta}$, and let $\tau_{\overline{\mathcal{X}}_{i}}$ be the first hitting time of the local Markov chain $\left(\bar{\eta}_{t}\right)_{t \geqslant 0}$ to the set $\overline{\mathcal{X}}_{i} \quad(i=1,2,3)$. The following proposition is our main recurrence result, giving an upper bound on $\tau_{\mathcal{X}_{i}}$.

Proposition 5.20: (“ $\overline{\mathcal{X}}_{i}$-recurrence”) For every $\delta>0$ there exists $\beta_{0}>0$ such that, for all $\beta$ $>\beta_{0}$,

$$
\max _{\bar{\eta} \in \mathcal{X} \overline{\mathcal{X}}_{i}} \mathrm{P}_{\bar{\eta}}\left(\tau_{\mathcal{X}_{i}}>T_{i} e^{\delta \beta}\right)=\operatorname{SES} \quad(i=1,2,3)
$$

Proof: We want to apply Proposition 2.13. Our task therefore is to define, for each $i$ $=1,2,3$, an event $\mathbf{E}_{T}^{i} \subset \overline{\mathcal{X}}^{[0, T)}$ with $T=T_{i} e^{(\delta / 2) \beta}$ such that

$$
\begin{gathered}
\mathbf{E}_{T}^{i} \subset\left\{\exists t \in[0, T]: \bar{\eta}_{t} \in \overline{\mathcal{X}}_{i}\right\}, \\
\min _{\mathbb{P}_{\bar{\eta}_{0}}}\left(\mathbf{E}_{T}^{i}\right) \geqslant p>0
\end{gathered}
$$

with $p$ not exponentially small in $\beta$.

(1) As noted in Remark (2) following Definition 5.1:

For each $\bar{\eta} \in \overline{\mathcal{X}} \backslash \overline{\mathcal{X}}_{1}$ there exists a finite 0-path $\phi: \bar{\eta} \rightarrow \bar{\eta}^{\prime} \in \overline{\mathcal{X}}_{1}$.

For each $\bar{\eta} \in \overline{\mathcal{X}} \backslash \overline{\mathcal{X}}_{2}$ there exists a finite $U$-path $\phi: \bar{\eta} \rightarrow \bar{\eta}^{\prime} \in \overline{\mathcal{X}}_{2}$.

For each $\bar{\eta} \in \overline{\mathcal{X}} \backslash \overline{\mathcal{X}}_{3}$ there exists a finite $\Delta$-path $\phi: \bar{\eta} \rightarrow \bar{\eta}^{\prime} \in \overline{\mathcal{X}}_{3}$.

If $\phi$ is a 0 -path, i.e., $\bar{H}\left(\phi_{i+1}\right) \leqslant \bar{H}\left(\phi_{i}\right)$ for all $i$, then for every $\delta>0$ there exist $\beta_{0}>0$ and $a$ $=a(\delta)>0$ such that, for all $\beta>\beta_{0}$,

$$
\mathbb{P}_{\bar{\eta}}\left(\bar{\eta}_{s}=\phi_{s} \forall s \in\left[0, T_{1}\right]\right) \geqslant a .
$$

(2) We want to have a similar statement for $U$-paths and $\Delta$-paths. To that end we make the following observation, valid for cycles $\mathcal{C}$.

Proposition 5.24: (i) For every $\delta>0$ there exist $\beta_{0}>0$ and $\kappa>0$ such that, for all $\beta>\beta_{0}$ and $\bar{\eta} \in \mathcal{C}$,

$$
\mathbb{P}_{\bar{\eta}}\left(\tau_{\partial \mathcal{C}}<e^{[H(\mathcal{F}(\partial \mathcal{C}))-H(\mathcal{F}(\mathcal{C}))+\delta] \beta}\right) \geqslant 1-e^{-\kappa \beta} .
$$

(ii) There exist $\delta_{0}>0, \beta_{0}>0$ and $\kappa>0$ such that, for all $\beta>\beta_{0}$ and $\bar{\eta}, \bar{\eta}^{\prime} \in \mathcal{C}$,

$$
\mathbb{P}_{\bar{\eta}}\left(\tau_{\bar{\eta}^{\prime}}<\tau_{\partial \mathcal{C}}, \tau_{\bar{\eta}^{\prime}}<e^{\left[H(\mathcal{F}(\partial \mathcal{C}))-H(\mathcal{F}(\mathcal{C}))-\delta_{0}\right] \beta}\right) \geqslant 1-e^{-\kappa \beta} .
$$

(iii) For every $\delta>0$ there exists $\beta_{0}>0$ such that, for all $\beta>\beta_{0}$ and $\bar{\eta} \in \mathcal{C}, \bar{\eta}^{\prime} \in \partial \mathcal{C}$,

$$
\mathrm{P}_{\bar{\eta}}\left(\bar{\eta}_{\tau_{\partial \mathcal{C}}}=\bar{\eta}^{\prime}\right) \geqslant e^{-\left[H\left(\bar{\eta}^{\prime}\right)-H(\mathcal{F}(\partial \mathcal{C}))+\delta\right] \beta} .
$$

Proof: See Olivieri and Scoppola ${ }^{10}$ Proposition 3.7. QED 
It follows from Proposition 5.24 (i) and (iii) that for every $\delta>0$ and every $U$-path $\phi$ there exist $\beta_{0}>0$ and $a=a(\delta)>0$ such that, for all $\beta>\beta_{0}$,

$$
\mathbb{P}_{\bar{\eta}}\left(\bar{\eta}_{s}=\phi \quad \forall s \in\left[0, T_{2} e^{\delta \beta}\right)\right) \geqslant a
$$

and similarly for $\Delta$-paths with $T_{2}$ replaced by $T_{3}$. So we have indeed generalized (5.23).

(3) To conclude the proof of Proposition 5.20 we now pick $\bar{\eta} \in \overline{\mathcal{X}} \backslash \overline{\mathcal{X}}_{i}$ and take for $\mathbf{E}_{T}^{i}$ the event where the process $\left(\bar{\eta}_{t}\right)_{t \geqslant 0}$ follows the $0, U, \Delta$-path from $\bar{\eta}$ to $\overline{\mathcal{X}}_{i}$ within time $T$ $=T_{i} e^{(\delta / 2) \beta}$. By (5.23) and (5.28), we have $\min _{\bar{\eta} \in \mathcal{X} \backslash \mathcal{X}_{i}} \mathrm{P}_{\bar{\eta}}\left(\mathbf{E}_{T}^{i}\right) \geqslant e^{-\delta^{\prime} \beta}$ with $\delta^{\prime}<\delta / 2$. Hence Proposition 2.13 gives

$$
\max _{\overline{\bar{\eta}} \in \overline{\mathcal{X}} \backslash \overline{\mathcal{X}}_{i}} \mathbb{P}_{\bar{\eta}}\left(\tau_{\mathcal{X}_{i}}>T_{i} e^{\delta \beta}\right) \leqslant\left(1-e^{-\delta^{\prime} \beta}\right)^{e^{(\delta / 2) \beta}}=\text { SES }
$$

QED

\subsection{Additional results on cycles}

In this section we collect some results on cycles and their relation to the recurrence sets $\overline{\mathcal{X}}_{i}$. These results will be needed in Sec. 7 .

Lemma 5.30: (i) If $\bar{\eta}_{1}, \bar{\eta}_{2} \in \overline{\mathcal{X}}_{1}$ with $\bar{H}\left(\bar{\eta}_{1}\right)<\bar{H}\left(\bar{\eta}_{2}\right)$, then $\bar{H}\left(\bar{\eta}_{1}, \bar{\eta}_{2}\right)-\bar{H}\left(\bar{\eta}_{2}\right) \geqslant U$.

(ii) If $\bar{\eta}_{1}, \bar{\eta}_{2} \in \overline{\mathcal{X}}_{2}$ with $\bar{H}\left(\bar{\eta}_{1}\right)<\bar{H}\left(\bar{\eta}_{2}\right)$, then $\bar{H}\left(\bar{\eta}_{1}, \bar{\eta}_{2}\right)-\bar{H}\left(\bar{\eta}_{2}\right) \geqslant \Delta$.

Proof: Note that the two smallest positive values for $\bar{H}\left(\bar{\eta}_{1}, \bar{\eta}_{2}\right)-\bar{H}\left(\bar{\eta}_{2}\right)$ are $U$ and $\Delta$.

(i) If $\bar{\eta}_{1}, \bar{\eta}_{2} \in \overline{\mathcal{X}}_{1}$ with $\bar{H}\left(\bar{\eta}_{1}\right)<\bar{H}\left(\bar{\eta}_{2}\right)$, then $\bar{\eta}_{1} \notin \overline{\mathcal{C}}_{\bar{\eta}_{2}}^{U}$ because otherwise $\bar{\eta}_{2}$ were 0 -reducible. But then $\bar{H}\left(\bar{\eta}_{1}, \bar{\eta}_{2}\right)-\bar{H}\left(\bar{\eta}_{2}\right) \geqslant U$.

(ii) If $\bar{\eta}_{1}, \bar{\eta}_{2} \in \overline{\mathcal{X}}_{2}$ with $\bar{H}\left(\bar{\eta}_{1}\right)<\bar{H}\left(\bar{\eta}_{2}\right)$, then $\bar{\eta}_{1} \notin \overline{\mathcal{C}}_{\bar{\eta}_{2}}^{\Delta}$ because otherwise $\bar{\eta}_{2}$ were $U$-reducible. But then $\bar{H}\left(\bar{\eta}_{1}, \bar{\eta}_{2}\right)-\bar{H}\left(\bar{\eta}_{2}\right) \geqslant \Delta$. QED

For the lacunary squares or quasi-squares in $\overline{\mathcal{X}}_{3}$ [recall Remark (2) in Sec. 5.2] we can prove the following.

Lemma 5.31: Let $\bar{\eta} \in \mathcal{L}$ (i.e., $\bar{\eta}$ is a lacunary square or quasi-square). Then there exists a $\bar{\eta}^{\prime} \in \overline{\mathcal{X}}_{3} \backslash \mathcal{L}$ with $\bar{H}\left(\bar{\eta}^{\prime}\right)<\bar{H}(\bar{\eta})$ such that there exists a $2 U$-path from $\bar{\eta}$ to $\bar{\eta}^{\prime}$.

Proof: We define an external corner of the internal structure of an element of $\mathcal{L}$ as a site $x$ $\in \bar{\Lambda}_{0}$ such that $\eta(x)=0, \Sigma_{y \in n n(x)} \eta(y)=2$ and such that there exists a path along occupied sites in $\bar{\eta}$ from $x$ to the external boundary $\partial \bar{\eta}$. The following $2 U$-path goes from $\bar{\eta}$ to $\bar{\eta}^{\prime}$ and reduces the energy:

- $\phi_{0}=\overline{\mathcal{C}}_{\bar{\eta}}^{2 U}$

- $\phi_{1}, \ldots, \phi_{t_{1}}$ is the sequence of configurations in which a hole that is an external corner of the internal structure of $\bar{\eta}$ goes to $\partial \bar{\eta}$;

- $\phi_{t_{1}+1}, \ldots, \phi_{t_{2}}$ is a $\Delta$-path going to $\overline{\mathcal{X}}_{3}$;

- $\phi_{t_{2}+1}=\overline{\mathcal{C}}_{\bar{\eta} t_{2}}^{2 U}$;

- $\phi_{t_{2}+2}, \ldots, \phi_{t_{3}}$ is the sequence of configurations in which a hole that is an external corner of the internal structure of $\bar{\eta}_{t_{2}}$ goes to $\partial \bar{\eta}_{t_{2}}$;

-and so on, until the complete removal of the internal structure.

QED

For $\bar{\eta} \in \overline{\mathcal{X}}_{3} \backslash \mathcal{L}, \bar{\eta}=R_{l_{1}, l_{2}}$, define

$$
\overline{\mathcal{C}}_{\bar{\eta}}=\left\{\bar{\eta}^{\prime} \in \overline{\mathcal{X}}: \bar{H}\left(\bar{\eta}^{\prime}, \bar{\eta}\right)-\bar{H}(\bar{\eta})<r(\bar{\eta})+\Delta\right\},
$$

where $r(\bar{\eta})$ is defined by 


$$
\begin{gathered}
l_{1}<l_{c}: r(\bar{\eta})=(2 U-\Delta)\left(l_{1}-1\right), \\
l_{1} \geqslant l_{c}: \quad r(\bar{\eta})=\Delta-U .
\end{gathered}
$$

The structure of this set is characterized as follows (compare with Lemma 5.13).

Lemma 5.34: (i) $\overline{\mathcal{C}}_{\bar{\eta}}$ is the maximal connected component containing $\bar{\eta}$ of configurations $\bar{\eta}^{\prime}$ such that $\bar{H}\left(\bar{\eta}^{\prime}\right)<\bar{H}(\bar{\eta})+r(\bar{\eta})+\Delta$.

(ii) If $\bar{\eta}^{\prime} \in \overline{\mathcal{C}}_{\bar{\eta}} \cap\left(\overline{\mathcal{X}}_{3} \backslash \mathcal{L}\right)$, then $\bar{\eta}^{\prime} \in \mathcal{E}_{3}(\bar{\eta})$ and $\bar{H}\left(\bar{\eta}^{\prime}\right)=\bar{H}(\bar{\eta})$.

(iii) If $\bar{\eta}^{\prime} \in \overline{\mathcal{C}}_{\bar{\eta}} \cap \mathcal{L}$, then $\overline{\mathcal{C}}_{\bar{\eta}^{\prime}}^{2 U} \subset \overline{\mathcal{C}}_{\bar{\eta}}$ and $\bar{H}\left(\bar{\eta}^{\prime}\right)>\bar{H}(\bar{\eta})$.

(iv) $\bar{\eta} \in \mathcal{F}\left(\overline{\mathcal{C}}_{\bar{\eta}}\right)$.

Proof: (i) Let $\overline{\mathcal{D}}_{\bar{\eta}}$ be the maximal connected component containing $\bar{\eta}$ of configurations $\bar{\eta}^{\prime}$ such that $\bar{H}\left(\bar{\eta}^{\prime}\right)<\bar{H}(\bar{\eta})+r(\bar{\eta})+\Delta$. If $\bar{\eta}^{\prime} \in \overline{\mathcal{C}}_{\bar{\eta}}$, then $\bar{\eta}^{\prime} \in \overline{\mathcal{D}}_{\bar{\eta}}$. Indeed, $\bar{H}\left(\bar{\eta}^{\prime}\right) \leqslant \bar{H}\left(\bar{\eta}^{\prime}, \bar{\eta}\right)$ and $\bar{\eta}^{\prime}$ is in the connected component because the trajectory realizing the minimax gives the connection. Conversely, if $\bar{\eta}^{\prime} \in \overline{\mathcal{D}}_{\bar{\eta}}$, then there exists a path $\phi: \bar{\eta} \rightarrow \bar{\eta}^{\prime}$ such that $\max _{i} \bar{H}\left(\phi_{i}\right)-\bar{H}(\bar{\eta})<r(\bar{\eta})$ $+\Delta$, while by the minimax definition we have $\bar{H}\left(\bar{\eta}, \bar{\eta}^{\prime}\right) \leqslant \max _{i} \bar{H}\left(\phi_{i}\right)$. Hence $\bar{H}\left(\bar{\eta}, \bar{\eta}^{\prime}\right)-\bar{H}(\bar{\eta})$ $<r(\bar{\eta})+\Delta$.

(ii) If $\bar{\eta}, \bar{\eta}^{\prime} \in \overline{\mathcal{X}}_{3} \backslash \mathcal{L}$, then by the saddle point results in Proposition 4.24 (i)-(ii) we have $\bar{H}\left(\bar{\eta}, \bar{\eta}^{\prime}\right)-\bar{H}(\bar{\eta}) \geqslant r(\bar{\eta})+\Delta$.

(iii) If $\bar{\eta}^{\prime} \in \overline{\mathcal{C}}_{\bar{\eta}} \cap \mathcal{L}$, then $\bar{H}\left(\bar{\eta}^{\prime}\right)+2 U-\bar{H}(\bar{\eta})<r(\bar{\eta})+\Delta$ by Lemma 5.13 . Hence $\overline{\mathcal{C}}_{\bar{\eta}^{\prime}}^{2 U} \subset \overline{\mathcal{C}}_{\bar{\eta}}$ and every $2 U$-path starting from $\bar{\eta}^{\prime}$ is contained in $\overline{\mathcal{C}}_{\bar{\eta}}$. But then, by Lemma 5.31, there exists a configuration $\bar{\eta}^{\prime \prime} \in \overline{\mathcal{X}}_{3} \backslash \mathcal{L}$ that is an element of $\overline{\mathcal{C}}_{\bar{\eta}}$. By (ii), this is possible only if $\bar{\eta}^{\prime \prime} \in \mathcal{E}_{3}(\bar{\eta})$, and in this case $\bar{H}\left(\bar{\eta}^{\prime}\right)>\bar{H}\left(\bar{\eta}^{\prime \prime}\right)=\bar{H}(\bar{\eta})$.

(iv) Suppose that there exists $\bar{\eta}^{\prime} \in \overline{\mathcal{C}}_{\bar{\eta}}$ such that $\bar{H}\left(\bar{\eta}^{\prime}\right)<\bar{H}(\bar{\eta})$. If $\bar{\eta}^{\prime} \notin \overline{\mathcal{X}}_{3}$, then there exists $\bar{\eta}^{\prime \prime} \in \overline{\mathcal{X}}_{3}$ with $\bar{H}\left(\bar{\eta}^{\prime \prime}\right)<\bar{H}\left(\bar{\eta}^{\prime}\right)$ and $\bar{H}\left(\bar{\eta}^{\prime}, \bar{\eta}^{\prime \prime}\right)-\bar{H}\left(\bar{\eta}^{\prime}\right) \leqslant \Delta$. If $\bar{\eta}^{\prime \prime} \in \overline{\mathcal{X}}_{3} \backslash \mathcal{L}$, then this contradicts (ii) If $\bar{\eta}^{\prime \prime} \in \mathcal{L}$, then by (iii) we have $\bar{H}\left(\bar{\eta}^{\prime \prime}\right)>\bar{H}(\bar{\eta})$, which contradicts the inequality $\bar{H}\left(\bar{\eta}^{\prime \prime}\right)$ $<\bar{H}\left(\bar{\eta}^{\prime}\right)<\bar{H}(\bar{\eta})$ coming from the reduction. QED

Remark: Throughout Secs. $4-5, \Delta$ is a parameter in $(U, 2 U)$ and $l_{c}(\Delta)=\lceil U /(2 U-\Delta)\rceil$. If $\Delta$ and $\Delta^{\prime}$ are such that $l_{c}(\Delta)=l_{c}\left(\Delta^{\prime}\right)$ and if we consider the two local Markov chains based on the values $\Delta$, resp., $\Delta^{\prime}$ for the creation rate along $\partial^{*} \bar{\Lambda}^{\text {in }}$ [recall (4.1)], then all the results obtained in Secs. 4-5 for these two Markov chains are equal up to a correction where in all the exponents containing $\Delta$ an error term of order $\left|\Delta^{\prime}-\Delta\right|$ is added. This observation is needed in Sec. 7, where we will need to perturb $\Delta$. It also explains why in Theorem 1.53 we need to assume that $U /(2 U-\Delta)$ is not integer.

\section{FULL MARKOV CHAIN: RECURRENCE}

In this section we extend the definitions of $\overline{\mathcal{X}}_{1} \supset \overline{\mathcal{X}}_{2} \supset \overline{\mathcal{X}}_{3}$, which were used in Sec. 5 as the recurrence sets for the local Markov chain, to $\mathcal{X}_{1} \supset \mathcal{X}_{2} \supset \mathcal{X}_{3}$ and we prove the recurrence properties of the full Markov chain $\left(\eta_{t}\right)_{t \geqslant 0}$ to these sets.

The sets $\mathcal{X}_{i}(i=1,2,3)$ are defined as follows [recall (2.27)]:

$$
\begin{aligned}
& \mathcal{X}_{1}=\left\{\eta \in \mathcal{X}_{0}:\left.\eta\right|_{\bar{\Lambda}} \in \overline{\mathcal{X}}_{1}\right\}, \\
& \mathcal{X}_{2}=\left\{\eta \in \mathcal{X}_{0}:\left.\eta\right|_{\bar{\Lambda}} \in \overline{\mathcal{X}}_{2}\right\}, \\
& \mathcal{X}_{3}=\left\{\eta \in \mathcal{X}_{0}:\left.\eta\right|_{\bar{\Lambda}} \in \overline{\mathcal{X}}_{3} \cap N_{\Lambda_{l_{-}} \backslash \bar{\Lambda}}(\eta)=0\right\} .
\end{aligned}
$$

Proposition 6.2 below shows that, up to a superexponentially small probability, the process $\left(\eta_{t}\right)_{t \geqslant 0}$ returns to $\mathcal{X}_{i}$ after time lapses of order $T_{i}$. 
Proposition 6.2: (“ $\mathcal{X}_{i}$-recurrence") Let $T_{1}=e^{0 \beta}, T_{2}=e^{U \beta}, T_{3}=e^{\Delta \beta}$. For $i=1,2,3$ the following estimate holds: There exist $\delta_{1}=\delta_{1}(\gamma)>0$, satisfying $\lim _{\gamma \downarrow 0} \delta(\gamma)=0$ and $\beta_{0}>0$ such that, for all $\beta>\beta_{0}$,

$$
\mathbb{P}_{\nu}\left(\exists t \in\left[0, T^{\prime \prime}\right): \eta_{s} \notin \mathcal{X}_{i} \quad \forall s \in\left[t, t+T_{i}^{\prime}\right)\right)=\mathrm{SES}
$$

for all

$$
T_{i}^{\prime}=T_{i} e^{\delta \beta} \ll T^{\prime \prime}=e^{C \beta}
$$

with $C>0$ arbitrarily large.

Proof: We want to apply Proposition 2.13. Our task therefore is to define, for each $i$ $=1,2,3$, an event $\mathbf{E}_{T}^{i} \subset \mathcal{X}^{[0, T)}$ with $T=T_{i} e^{(\delta / 2) \beta}$ such that

$$
\begin{gathered}
\mathbf{E}_{T}^{i} \subset\left\{\exists t \in[0, T]: \eta_{t} \in \mathcal{X}_{i}\right\}, \\
\min _{\eta_{0} \in \mathcal{X}_{0}} \mathbb{P}_{\eta_{0}}\left(\mathbf{E}_{T}^{i}\right) \geqslant p>0
\end{gathered}
$$

with $p$ not exponentially small in $\beta$.

$i=1,2$. The event $\mathbf{E}_{T}^{i}$ is the following:

-During the time interval $[0, T]$ no particle enters $\bar{\Lambda}$.

-The process $\left(\eta_{t}\right)_{t \geqslant 0}$ restricted to $\bar{\Lambda}$ follows a 0 -path $(i=1)$, respectively, a $U$-path $(i=2)$ from $\eta_{0} \mid \bar{\Lambda}$ to $\overline{\mathcal{X}}_{i}$ within the time interval $[0, T]$.

Since $T=T_{i} e^{(\delta / 2) \beta} \leqslant e^{\Delta \beta-(\delta / 2) \beta}$ for $i=1,2$ and $\delta>0$ sufficiently small we have, by Propositions 3.20 and 3.25 ,

$$
\min _{\eta_{0} \in \mathcal{X}_{0}} \mathbb{P}_{\eta_{0}}(\text { during }[0, T] \text { no particles enter } \bar{\Lambda}) \geqslant\left(\frac{\kappa}{\beta}\right)^{\log \beta} \text {. }
$$

The estimate of $\mathrm{P}_{\eta_{0}}\left(\mathbf{E}_{T}^{i}\right)$ can therefore be completed by using (5.23), (5.28), Proposition 2.28, and Proposition 2.2.

$i=3$. We cannot proceed in the same way as for $i=1,2$, since we cannot avoid the arrival of particles over a time interval of length $T=T_{3} e^{(\delta / 2) \beta}$. Actually, the arrival of particles is important to reach $\mathcal{X}_{3}$. The event $\mathbf{E}_{T}^{3}$ is the following:

(i) The process $\left(\eta_{t}\right)_{t \geqslant 0}$ restricted to $\bar{\Lambda}$ follows a $\Delta$-path from $\left.\eta_{0}\right|_{\bar{\Lambda}}$ to $\overline{\mathcal{X}}_{3}$ within time $\frac{1}{2} T_{3} e^{(\delta / 2) \beta}$.

(ii) After that, the process within time $T_{3} e^{-(\gamma / 2) \beta}$ empties the annulus $\Lambda_{l_{-}} \backslash \bar{\Lambda}$, while keeping the configuration in $\bar{\Lambda}$ fixed and avoiding that particles enter $\bar{\Lambda}$.

To complete the proof we have to show that the probabilities of (i) and (ii) are not exponentially small.

Estimate of (i). For each segment of the $\Delta$-path not containing cycles of depth $\Delta$ we can prove (using the same argument as for $i=1,2$ ) that the probability for the process to follow this segment within time $T_{2} e^{(\delta / 2) \beta}$ is not exponentially small. We therefore only have to control the probability that the process follows the segments of the $\Delta$-path containing cycles of depth $\Delta$, i.e., we have to control the probability that it exits from the set

$$
C_{\bar{\eta}^{\prime}}^{\Delta}=\left\{\eta \in \mathcal{X}:\left.\eta\right|_{\Lambda} \in \overline{\mathcal{C}}_{\bar{\eta}^{\prime}}^{\Delta}\right\} \quad \text { for some } \bar{\eta}^{\prime} \in \overline{\mathcal{X}}_{2}
$$

within a time larger than $T_{3} e^{(\delta / 3) \beta}$ and that it exits at a configuration in $\mathcal{F}\left(\partial \overline{\mathcal{C}}_{\bar{\eta}^{\prime}}^{\Delta}\right)$. This is done by the following estimate. 
Lemma 6.8: For $\delta>0$ small enough and $0<\gamma<\delta / 3$ there exists $c>0$, depending on $\gamma, \delta$ but not on $\beta$, such that

$$
\min _{\eta \in C_{\bar{\eta}^{\prime}}^{\Delta} \cap \mathcal{X}_{0}} \mathbb{P}_{\eta}\left(\left\{\tau_{\left(C_{\bar{\eta}^{\prime}}^{\Delta}, c\right.}<T_{3} e^{(\delta / 3) \beta}\right\} \cap\left\{\eta_{\tau_{\left(C_{\bar{\eta}^{\prime}}\right.}^{\Delta} c} \mid \bar{\Lambda} \in \mathcal{F}\left(\partial \overline{\mathcal{C}}_{\bar{\eta}^{\prime}}^{\Delta}\right)\right\}\right) \geqslant c
$$

Proof: The proof uses a splitting of events.

(1) From Lemma 5.13 (ii) we know that if $\bar{\eta} \notin \mathcal{F}\left(\overline{\mathcal{C}}_{\bar{\eta}^{\prime}}^{\Delta}\right)$, then $\bar{\eta} \in \overline{\mathcal{X}} \backslash \overline{\mathcal{X}}_{1}$ and there exists a downhill path from $\bar{\eta}$ to $\mathcal{F}\left(\overline{\mathcal{C}}_{\bar{\eta}^{\prime}}^{\Delta}\right)$. We can therefore require that the process $\left(\eta_{t}\right)_{t \geqslant 0}$ follows this path within a time $e^{\delta \beta}$ and that during this time no particle enters $\bar{\Lambda}$. As before, this probability is not exponentially small in $\beta$. It is therefore sufficient to show that, for all $\eta_{0}^{\prime} \in \mathcal{X}_{0}$ such that $\left.\eta_{0}^{\prime}\right|_{\bar{\Lambda}} \in \mathcal{F}\left(\overline{\mathcal{C}}_{\bar{\eta}^{\prime}}^{\Delta}\right)$,

$$
\mathbb{P}_{\eta_{0}^{\prime}}\left(\left\{\tau_{\left(C_{\bar{\eta}^{\prime}}^{\Delta}\right)}<T_{3} e^{(\delta / 3) \beta}\right\} \cap\left\{\eta_{\tau_{\left(C_{\bar{\eta}^{\prime}}\right.}^{\Delta} d} \mid \bar{\Lambda} \in \mathcal{F}\left(\partial \overline{\mathcal{C}}_{\bar{\eta}^{\prime}}^{\Delta}\right)\right\}\right) \geqslant c .
$$

(2) Let $\tau$ denote the first time a particle enters $\bar{\Lambda}$. Then

$$
\mathbb{P}_{\eta_{0}^{\prime}}\left(\left\{\tau_{\left(C_{\bar{\eta}^{\prime}}^{\Delta} c\right.}^{\Delta}<T_{3} e^{(\delta / 3) \beta}\right\} \cap\left\{\eta_{\tau_{\left(C_{\bar{\eta}^{\prime}}\right.}^{\Delta} d} d \bar{\Lambda} \in \mathcal{F}\left(\partial \overline{\mathcal{C}}_{\bar{\eta}^{\prime}}^{\Delta}\right)\right\}\right) \geqslant \int_{0}^{T_{3} e^{(\delta / 3) \beta}} \mathbb{P}_{\eta_{0}^{\prime}}\left(\tau \in d t, \mathbf{A}_{t} \cap \mathbf{B}_{t}\right),
$$

where

$$
\begin{aligned}
& \mathbf{A}_{t}=\left\{\tau_{\left(\mathcal{C}_{\bar{\eta}^{\prime}}\right) c}^{\Delta}>t\right\}, \\
& \mathbf{B}_{t}=\left\{\left.\eta_{t^{-}}\right|_{\bar{\Lambda}} \in \mathcal{F}\left(\overline{\mathcal{C}}_{\bar{\eta}^{\prime}}^{\Delta}\right)\right\} .
\end{aligned}
$$

The rhs of (6.11) equals

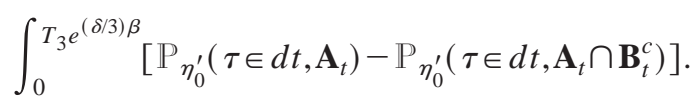

Due to the recurrence property in $\mathcal{X}_{1}$ and due to the fact that $\overline{\mathcal{C}}_{\bar{\eta}^{\prime}}^{\Delta} \backslash \mathcal{F}\left(\overline{\mathcal{C}}_{\bar{\eta}^{\prime}}^{\Delta}\right) \subset \overline{\mathcal{X}} \backslash \overline{\mathcal{X}}_{1}$ [recall Lemma 5.13 (ii)], we have that on the event $\left\{\tau \in d t, \mathbf{A}_{t} \cap \mathbf{B}_{t}^{c}\right\}$ a move of cost $U$ occurs during the time interval $\left[\left(t-e^{\delta \beta}\right) \vee 0, t\right]$. Hence $\mathbb{P}_{\eta_{0}^{\prime}}\left(\mathbf{A}_{t} \cap \mathbf{B}_{t}^{c} \mid \tau \in d t\right) \leqslant e^{-(U-\delta) \beta}$, and so

$$
\int_{0}^{T_{3} e^{(\delta / 3) \beta}} \mathbb{P}_{\eta_{0}^{\prime}}\left(\tau \in d t, \mathbf{A}_{t} \cap \mathbf{B}_{t}^{c}\right) \leqslant e^{-(U-\delta) \beta} .
$$

On the other hand, since on the event $\left\{\tau \in d t, \mathbf{A}_{t}^{c}\right\}$ a move of cost $2 U$ occurs within time $t$, we have

$$
\begin{aligned}
\int_{0}^{T_{3} e^{(\delta / 3) \beta}} \mathrm{P}_{\eta_{0}^{\prime}}\left(\tau \in d t, \mathbf{A}_{t}\right) & =\int_{0}^{T_{3} e^{(\delta / 3) \beta}}\left[\mathbb{P}_{\eta_{0}^{\prime}}(\tau \in d t)-\mathbb{P}_{\eta_{0}^{\prime}}\left(\tau \in d t, \mathbf{A}_{t}^{c}\right)\right] \\
& \geqslant \int_{0}^{T_{3} e^{(\delta / 3) \beta}} \mathrm{P}_{\eta_{0}^{\prime}}(\tau \in d t)-e^{-(2 U-\Delta-(\delta / 3)) \beta} .
\end{aligned}
$$

(3) Finally, we use that $\left\{\eta_{0}^{\prime} \in \mathcal{X}_{0}\right\} \subset\left\{N_{\Lambda_{l_{+}}}\left(\eta_{0}^{\prime}\right) \geqslant 1\right\}$ [recall (2.26)]. If $\delta / 3>\gamma$, then the probability for a particle starting in $\Lambda_{l_{+}}$to hit $\bar{\Lambda}$ prior to time $T_{3} e^{(\delta / 2) \beta}$ is bounded from below by some $c>0$, so that 


$$
\int_{0}^{T_{3} e^{(\delta / 3) \beta}} \mathbb{P}_{\eta_{0}^{\prime}}(\tau \in d t)=\mathbb{P}_{\eta_{0}^{\prime}}\left(\tau \leqslant T_{3} e^{(\delta / 3) \beta}\right) \geqslant c .
$$

QED

Estimate of (ii). We estimate the probability of the following events:

F: during the time interval $\left[0, T_{3} e^{-(\gamma / 2) \beta}\right]$ no particle enters $\bar{\Lambda}$;

G: at time $T_{3} e^{-(\gamma / 2) \beta}$ there are no particles in $\Lambda_{l_{-}} \backslash \bar{\Lambda}$;

H: during the time interval $\left[0, T_{3} e^{-(\gamma / 2) \beta}\right]$ the configuration in $\bar{\Lambda}$ does not change.

From Propositions 3.20 and 3.25 we obtain

$$
\min _{\eta_{0}^{\prime} \in \mathcal{X}_{0}} \mathbb{P}_{\eta_{0}^{\prime}}(\mathbf{F} \cap \mathbf{G}) \geqslant\left(\frac{\kappa}{\beta}\right)^{\log \beta} .
$$

Moreover, $\mathbb{P}_{\eta_{0}^{\prime}}(\mathbf{H} \mid \mathbf{F} \cap \mathbf{G})$ can be estimated by considering the local Markov chain, and we obtain

$$
\min _{\eta_{0}^{\prime} \in \mathcal{X}_{0}: \eta_{0}^{\prime} \mid \bar{\Lambda} \in \overline{\mathcal{X}}_{3}} \mathbb{P}_{\eta_{0}^{\prime}}(\mathbf{H} \mid \mathbf{F} \cap \mathbf{G}) \geqslant\left(1-e^{-2 U \beta}\right)^{T_{3} e^{-(\gamma / 2) \beta}} \geqslant 1-e^{-[2 U-\Delta+(\gamma / 2)] \beta},
$$

because for all configurations in $\overline{\mathcal{X}}_{3}$ a move costs at least $2 U$ [see Proposition 5.11(iii)]. Combining (6.17)-(6.18), we arrive at

$$
\min _{\eta_{0}^{\prime} \in \mathcal{X}_{0}:\left.\eta_{0}^{\prime}\right|_{\bar{\Lambda}} \in \overline{\mathcal{X}}_{3}} \mathbb{P}_{\eta_{0}^{\prime}}(\mathbf{F} \cap \mathbf{G} \cap \mathbf{H}) \geqslant\left[1-e^{-[2 U-\Delta+(\gamma / 2)] \beta}\right]\left(\frac{\kappa}{\beta}\right)^{\log \beta} .
$$

This completes the proof of (i)-(ii) for $i=3$ and hence of Proposition 6.2. QED

\section{FULL MARKOV CHAIN: REDUCTION}

In this section we derive all the key estimates for the full Markov chain that are needed to study its metastable behavior. The computations are long and difficult.

\subsection{Definition of the reduced Markov chain}

We begin by defining the reduced Markov chain that is obtained by observing the process only when it enters $\mathcal{X}_{3}$ after it exits a suitable cycle of depth $\Delta$. For $\bar{\eta} \in \overline{\mathcal{X}}_{3}$, let

$$
\overline{\mathcal{C}}_{\bar{\eta}}^{\Delta}=\left\{\bar{\eta}^{\prime} \in \overline{\mathcal{X}}: \bar{H}\left(\bar{\eta}^{\prime}, \bar{\eta}\right)-\bar{H}(\bar{\eta})<\Delta\right\} .
$$

Extend this definition to the configurations in $\mathcal{X}$ in the obvious way: for $\eta \in \mathcal{X}_{3}$, let

$$
\mathcal{C}_{\eta}^{\Delta}=\left\{\eta^{\prime} \in \mathcal{X}:\left.\eta^{\prime}\right|_{\bar{\Lambda}} \in \overline{\mathcal{C}}_{\left.\eta\right|_{\Lambda}}^{\Delta}\right\}
$$

Definition 7.3: Let

$$
\begin{aligned}
\tau_{0} & =0, \\
\sigma_{0} & =\min \left\{t \geqslant 0: \eta_{t} \notin \mathcal{C}_{\eta_{0}}^{\Delta}\right\},
\end{aligned}
$$

and, for $i \in \mathbb{N}$,

$$
\tau_{i}=\min \left\{t>\sigma_{i-1}: \eta_{t} \in \mathcal{X}_{3}\right\}
$$




$$
\sigma_{i}=\min \left\{t>\tau_{i}: \eta_{t} \notin \mathcal{C}_{\eta_{\tau_{i}}}^{\Delta}\right\}
$$

Then

$$
\eta_{i}^{R}=\eta_{\tau_{i}}, \quad i \in \mathbb{N}_{0},
$$

defines a Markov chain $\left(\eta_{i}^{R}\right)_{i \in \mathbb{N}_{0}}$ on $\mathcal{X}_{3}$ with transition probabilities

$$
P^{R}\left(\eta, \eta^{\prime}\right)=\mathbb{P}_{\eta}\left(\eta_{\tau_{1}}=\eta^{\prime}\right) .
$$

For $\bar{\eta} \in \overline{\mathcal{X}}$, as before, let $I_{\bar{\eta}}$ be the set of configurations $\eta^{\prime} \in \mathcal{X}$ such that $\left.\eta^{\prime}\right|_{\bar{\Lambda}}=\bar{\eta}$. Note that $I_{\bar{\eta}}$ is not a subset of $\mathcal{X}_{3}$. By an abuse of notation, for $\eta \in \mathcal{X}_{3}$ and $\bar{\eta}^{\prime} \in \overline{\mathcal{X}}_{3}$ we write

$$
P^{R}\left(\eta, I_{\bar{\eta}^{\prime}}\right)=\sum_{\eta^{\prime \prime} \in I_{\bar{\eta}^{\prime}} \cap \mathcal{X}_{3}^{\prime}} P^{R}\left(\eta, \eta^{\prime \prime}\right) .
$$

Our main result in this section is the following proposition, which makes a comparison between the transition probabilities of the full Markov chain and the local Markov chain. Recall that $\gamma$ is the exponent in Propositions 2.17, 2.20, and 2.23.

Proposition 7.9: There exist $\delta=\delta(\gamma)$, satisfying $\lim _{\gamma \downarrow 0} \delta(\gamma)=0$, and $\beta_{0}>0$ such that for all $\beta>\beta_{0}$ :

(i) If $\bar{\eta} \in \mathcal{R}_{l_{1}, l_{2}}, \bar{\eta}^{\prime} \in \mathcal{R}_{l_{1}+1}, l_{2}$, then

$$
\min _{\eta \in I_{\bar{\eta}} \cap \mathcal{X}_{3}} P^{R}\left(\eta, I_{\bar{\eta}^{\prime}}\right) \geqslant e^{-(\Delta-U+\delta) \beta} .
$$

(ii) If $\bar{\eta} \in \mathcal{R}_{l_{1}, l_{2}}, \bar{\eta}^{\prime} \in \mathcal{R}_{l_{1}, l_{2}-1}$, then

$$
\min _{\eta \in I_{\bar{\eta}} \cap \mathcal{X}_{3}} P^{R}\left(\eta, I_{\bar{\eta}^{\prime}}\right) \geqslant e^{-\left[(2 U-\Delta)\left(l_{1}-1\right)+\delta\right] \beta} .
$$

(iii) If $\bar{\eta}$ is a lacunary square or quasi-square, i.e., $\bar{\eta} \in \mathcal{L}$, then there exists a sequence $\bar{\eta}_{0}, \bar{\eta}_{1}, \ldots, \bar{\eta}_{n}(n \in \mathbb{N})$ such that $\bar{\eta}_{0}=\bar{\eta}, \bar{\eta}_{i} \in \mathcal{L}$ for $i=1, \ldots, n-1, \bar{\eta}_{n} \in \overline{\mathcal{X}}_{3} \backslash \mathcal{L}$, and $\left|\bar{\eta}_{i}\right| \geqslant\left|\bar{\eta}_{i-1}\right|$ for all $i=1, \ldots, n-1$ for which

$$
\min _{i=0,1, \ldots, n-1} \min _{\eta_{i} \in I_{\bar{\eta}_{i}} \cap \mathcal{X}_{3}} P^{R}\left(\eta_{i}, I_{\bar{\eta}_{i+1}}\right) \geqslant e^{-(2 U-\Delta+\delta) \beta} .
$$

(iv) Let

$$
\begin{array}{ll}
r(\bar{\eta})=(2 U-\Delta)\left(l_{1}-1\right) & \text { if } \bar{\eta}=R_{l_{1}, l_{2}} \text { and } 0 \leqslant l_{2}-l_{1} \leqslant 1 \text { with } l_{1}<l_{c}, \\
r(\bar{\eta})=\Delta-U & \text { if } \bar{\eta}=R_{l_{1}, l_{2}} \text { and } 0 \leqslant l_{2}-l_{1} \leqslant 1 \text { with } l_{1} \geqslant l_{c}, \\
r(\bar{\eta})=(2 U-\Delta) & \text { if } \bar{\eta} \in \mathcal{L} .
\end{array}
$$

Then

$$
\max _{\eta \in I_{\bar{\eta}}} \max _{\bar{\eta}^{\prime} \in \overline{\mathcal{X}}_{3}: \bar{\eta}^{\prime} \notin \mathcal{E}_{3}(\bar{\eta})} P^{R}\left(\eta, I_{\bar{\eta}^{\prime}}\right) \leqslant e^{-[r(\bar{\eta})-\delta] \beta} .
$$

Using Proposition 7.9 we will in Sec. 8 conclude the proof of our main result, Theorem 1.53, by constructing an event of nucleation and by controlling its probability. 
Note that $r(\bar{\eta})=\min _{\bar{\eta}^{\prime} \in \overline{\mathcal{X}}_{\backslash \backslash \mathcal{E}}(\bar{\eta})} \bar{H}\left(\bar{\eta}, \bar{\eta}^{\prime}\right)-\Delta$ for every $\bar{\eta} \in \overline{\mathcal{X}}_{3}$. The $\Delta$ comes from the fact that, by definition, a transition of the reduced Markov chain typically takes place after a time of order $T_{3}=e^{\Delta \beta}$.

\subsection{Outline}

The remainder of this section is organized as follows. In Sec. 7.3 we prove the lower bounds in Proposition 7.9 (i)-(iii). The proof of the upper bound in Proposition 7.9 (iv) is much more difficult. Indeed, to prove a lower bound it suffices to estimate the probability of a particular realization of the event we are considering, but to prove an upper bound every possible realization of the event must be controlled.

In the estimate for the upper bound we have to study in detail the behavior of the gas and its interaction with the dynamics in the box $\bar{\Lambda}$. It is clear that there are two classes of gas particles with different behavior: (1) particles that have been in $\Lambda_{\beta} \backslash \bar{\Lambda}$ for a long time (say of order $T_{3}$ ), which we call green particles; (2) particles that exit from $\bar{\Lambda}$ and afterwards return to $\bar{\Lambda}$ in a short time (say of order 1), which we call red particles. We study separately the effect of green and red particles by introducing an auxiliary Markov chain $\left(\tilde{\eta}_{t}\right)_{t \geqslant 0}$ in which the arrival at $\bar{\Lambda}$ of green particles is simulated with a creation mechanism on $\partial^{-} \bar{\Lambda}$. In Sec. 7.4 we define the coloring and the auxiliary Markov chain, as well as a coupling between the auxiliary Markov chain and $\left(\eta_{t}\right)_{t \geqslant 0}$. The effect of green particles is studied in Sec. 7.6 by comparing the two processes.

The difficult part is developed in Sec. 7.7, where the effect of red particles is studied. In particular, we have to control the red particles when the process exits a suitable cylindrical set $\mathcal{C}_{\bar{\eta}}$, with base in $\bar{\Lambda}$ (see Proposition 7.43). The idea is the following:

-We analyze the cylindrical set by using the state classification $\overline{\mathcal{X}}_{1}, \overline{\mathcal{X}}_{2}, \overline{\mathcal{X}}_{3}$ (Lemma 7.69) and obtain in this way a partition of the set $\mathcal{C}_{\bar{\eta}}$ and of the different ways of exit from this set in terms of the final exiting move.

-Using this partition, we classify the intervals of time spent by the process in the different elements of the partition, by defining the "type" of the time interval in terms of the index of the corresponding element of the partition. In particular, the time intervals corresponding to times spent outside $\overline{\mathcal{X}}_{2}$ are called "instability intervals." We can easily control the length of instability intervals by using the recurrence property (Lemma 7.75).

-It turns out that, before the process exits the set $\mathcal{C}_{\bar{\eta}}$, red particles cannot arrive during an instability interval, since their entry in $\bar{\Lambda}$ would increase the energy and produce the exit of the process from $\mathcal{C}_{\bar{\eta}}$. This means that the effect of red particles before the exit from $\mathcal{C}_{\bar{\eta}}$ can be essentially described by the appearence of instability intervals starting with the arrival of a red particle. If at the end of such an instability interval a particle exits from $\bar{\Lambda}$, then we can "glue together" the red particles at the entry and at the exit, thus obtaining the path of a single red particle that spends some time in $\bar{\Lambda}$ (the instability interval). On the other hand, if at the beginning of an instability interval no red particle enters $\bar{\Lambda}$ and at the end a particle leaves $\bar{\Lambda}$, then we call the beginning of such an interval a "coloration time $\alpha_{k}$ " of a red particle. If an instability interval begins with the arrival of a red particle but ends with no particle leaving $\bar{\Lambda}$, then in this time interval a red particle has been absorbed by the box $\bar{\Lambda}$.

By following this kind of construction, we can associate with each red particle a coloration time $\alpha_{k}$, a "quasi random walk" obtained by glueing together the trajectories of red particles entering and exiting during the same instability interval, and a "delay time" corresponding to the sum of instability intervals visited by this path.

We show that the behavior of quasi random walks is similar to that of random walks, and in this way we control the total number of instability intervals of each type by estimating the number

of visits to $\bar{\Lambda}$ of quasi random walks (Lemma 7.91). This control on the behavior of quasi random 
walks enables us also to estimate from above the probability that a red particle arrives in $\bar{\Lambda}$ in a given time interval (Lemma 7.93).

-The effect of red particles in the exit of the process from the set $\mathcal{C}_{\bar{\eta}}$ is then controlled by introducing this probability estimate in the analysis of the possible ways of exit, as mentioned above.

Section 7.8 contains the proofs of some lemmas that are needed along the way. In Sec. 7.9 we collect some consequence of Proposition 7.9 that will be used in Sec. 8 to prove our main theorem.

\subsection{Lower bounds}

In this section we prove Proposition 7.9 (i)-(iii).

Estimate (i). The proof follows the same technique of construction of events as in Sec. 6.

(1) Let $\bar{\eta}_{i}, 0 \leqslant i \leqslant l_{2}$, be the configurations with monotone contour obtained from $R_{l_{1}+1, l_{2}}$, by removing $l_{2}-i$ particles, so that $\bar{\eta}_{0}=\bar{\eta}$ and $\bar{\eta}_{l_{2}}=\bar{\eta}^{\prime}$. We know from Sec. 5 that $\bar{\eta}_{i} \in \overline{\mathcal{X}}_{2}$ for $i$ $=0,2, \ldots, l_{2}$. We construct our realization of the growing transition $\bar{\eta}=R_{l_{1}, l_{2}} \rightarrow \bar{\eta}^{\prime}=R_{l_{1}+1, l_{2}}$ by using the following events:

For $i=1:$
$-\mathbf{A}_{1, t}=\left\{\tau_{\left(I_{\bar{\eta}}\right)^{c}} \geqslant t\right\} ;$
$-\mathbf{B}_{1, t}=\left\{\left.\eta_{t^{-}}\right|_{\bar{\Lambda}}=\bar{\eta}\right\} ;$

-for $\eta^{\prime \prime} \in I_{\bar{\eta}}$ and $x \in \partial \bar{\Lambda}, \mathbf{C}_{1, \eta^{\prime \prime}, x}$ is the event where the process, starting from $\eta^{\prime \prime}+x$, inside $\bar{\Lambda}$ follows a path that brings the particle at $x$ to the cluster, thus reaching $I_{\bar{\eta}_{1}}$.

For $i=2$ :

- $\mathbf{A}_{2, t}=\left\{\tau_{\left(\mathcal{C}_{\bar{\eta}}^{2 U}\right) c} \geqslant t\right\}$;

$\cdot \mathbf{B}_{2, t}=\left\{\left.\eta_{t^{-}}\right|_{\bar{\Lambda}} \in \overline{\mathcal{B}}_{\bar{\eta}}\right\}$ with $\overline{\mathcal{B}}_{\bar{\eta}}=\overline{\mathcal{C}}_{\bar{\eta}}^{2 U} \cap \overline{\mathcal{X}}_{1} \cap\left\{\bar{\eta}^{\prime \prime}: N_{\bar{\Lambda}}\left(\bar{\eta}^{\prime \prime}\right)=l_{1} l_{2}+1\right\}$;

-for $\eta^{\prime \prime} \in I_{\overline{\mathcal{B}}_{\bar{\eta}}}$ and $x \in \partial \bar{\Lambda}, \mathbf{C}_{2, \eta^{\prime \prime}, x}$ is the event where the process, starting at $\eta^{\prime \prime}+x$, inside $\bar{\Lambda}$ follows a path that takes the particle at $x$ to the cluster, thus reaching $I_{\bar{\eta}_{2}}$.

For $i=3, \ldots, l_{2}$ :

$\cdot \mathbf{A}_{i, t}=\left\{\tau_{\left(\mathcal{C}_{-1}^{\Delta}\right)} \geqslant t\right\}$;

$\cdot \mathbf{B}_{i, t}=\left\{\left.\eta_{t^{-}}\right|_{\bar{\Lambda}} \in \mathcal{F}\left(\mathcal{C}_{\bar{\eta}_{i-1}}^{\Delta}\right)\right\}$;

-for $\eta^{\prime \prime} \in I_{\mathcal{F}\left(\mathcal{C}_{\bar{\eta}_{i-1}}^{\Delta}\right)}$ and $x \in \partial \bar{\Lambda}, \mathbf{C}_{i, \eta^{\prime \prime}, x}$ is the event where the process, starting at $\eta^{\prime \prime}+x$, inside $\bar{\Lambda}$ follows a path that brings the particle at $x$ to the cluster, thus reaching $I_{\bar{\eta}_{i}}$.

(2) As in Sec. 6, we define

F: during the time interval $\left[0, T_{3} e^{-\left(\delta_{0} / 2\right) \beta}\right]$ no particle enters $\bar{\Lambda}$;

G: at time $T_{3} e^{-\left(\delta_{0} / 2\right) \beta}$ there are no particles in $\Lambda_{l_{-}} \backslash \bar{\Lambda}$;

H: during the time interval $\left[0, T_{3} e^{-\left(\delta_{0} / 2\right) \beta}\right]$ the configuration in $\bar{\Lambda}$ does not change.

Let $\tau=\min \{t \geqslant 0$ : at time $t$ a particle enters $\bar{\Lambda}\}$ and $T=T_{3} e^{\delta_{1} \beta} /\left(l_{1}+1\right)$, where $\delta_{1}$ is the parameter appearing in Proposition 6.2. For $\eta \in I_{\bar{\eta}}$ we have

$$
\begin{aligned}
& P^{R}\left(\eta, I_{\bar{\eta}^{\prime}}\right) \geqslant \\
& \int_{0}^{T} \mathbb{P}_{\eta}\left(\tau \in d t_{1}, \mathbf{A}_{1, t_{1}} \cap \mathbf{B}_{1, t_{1}}\right) \min _{x_{1} \in \partial \Lambda}{ }_{\bar{\Lambda}, \eta^{\prime \prime} \in I_{\bar{\eta}}} P_{\bar{\eta}^{\prime \prime}+x_{1}}\left(\mathbf{C}_{1, \eta^{\prime \prime}, x_{1}}\right) \\
& \int_{e^{U \beta}}^{T} \mathbb{P}_{\eta_{1}}\left(\tau \in d t_{2}, \mathbf{A}_{2, t_{2}} \cap \mathbf{B}_{2, t_{2}}\right) \min _{x_{2} \in \partial \bar{\Lambda}, \eta^{\prime \prime} \in I_{\mathcal{B}_{\bar{\eta}}}} \mathbb{P}_{\bar{\eta}^{\prime \prime}+x_{2}}\left(\mathbf{C}_{2, \eta^{\prime \prime}, x_{2}}\right)
\end{aligned}
$$




$$
\begin{gathered}
\int_{0}^{T} \mathbb{P}_{\eta_{i-1}}\left(\tau \in d t_{i}, \mathbf{A}_{i, t_{i}} \cap \mathbf{B}_{i, t_{i}}\right) \min _{x_{i} \in \partial \bar{\Lambda}, \eta^{\prime \prime} \in I_{\mathcal{F}\left(C_{\bar{\eta}_{i-1}}^{\Delta}\right.}} \mathbb{P}_{\bar{\eta}^{\prime \prime}+x_{i}}\left(C_{i, \eta^{\prime \prime}, x_{i}}\right) \\
\ldots \\
\ldots \\
\int_{0}^{T} \mathbb{P}_{\eta_{l_{2}-1}}\left(\tau \in d t_{l_{2}}, \mathbf{A}_{l_{2}, t_{l_{2}}} \cap \mathbf{B}_{l_{2}, t_{l_{2}}}\right) \min _{\left.x_{l_{2}} \in \partial \bar{\Lambda}, \eta^{\prime \prime} \in I_{\mathcal{F}\left(c_{l_{2-1}}^{\Delta}\right.}^{\Delta}\right)} \mathbb{P}_{\bar{\eta}^{\prime \prime}+x_{l_{2}}}\left(\mathbf{C}_{l_{2}, \eta^{\prime \prime}, x_{l_{2}}}\right) \\
\min _{\eta^{\prime \prime} \in I_{\bar{\eta}^{\prime}} \cap \mathcal{X}_{0}} \mathbb{P}_{\eta^{\prime \prime}}(\mathbf{F} \cap \mathbf{G} \cap \mathbf{H}),
\end{gathered}
$$

where $\mathcal{X}_{0}$ is the set defined in (2.27).

(3) For any $i$ we have that the minimum of the probability of $\mathbf{C}_{i, \eta^{\prime \prime}, x_{i}}$ can be estimated from below by a constant $a$ not exponentially small in $\beta$. As in Sec. 6 , for each $i=1,3, \ldots, l_{2}$ we can write

$$
\int_{0}^{T} \mathbb{P}_{\eta_{i-1}}\left(\tau \in d t_{i}, \mathbf{A}_{i, t_{i}} \cap \mathbf{B}_{i, t_{i}}\right)=\int_{0}^{T}\left[\mathbb{P}_{\eta_{i-1}}\left(\tau \in d t_{i}, \mathbf{A}_{i, t_{i}}\right)-\mathbb{P}_{\eta_{i-1}}\left(\tau \in d t_{i}, \mathbf{A}_{i, t_{i}} \cap \mathbf{B}_{i, t_{i}}^{c}\right)\right]
$$

and this expression can be estimated from below by a constant $c$ independent of $\beta$ exactly as in the proof of Lemma 6.8. Moreover, we again have that $\min _{\eta^{\prime \prime} \in I_{\bar{\eta}^{\prime}} \cap \mathcal{X}_{0}} \mathrm{P}_{\eta^{\prime \prime}}(\mathbf{F} \cap \mathbf{G} \cap \mathbf{H}) \geqslant(\kappa / \beta)^{\log \beta}$. It therefore remains to estimate the term corresponding to $i=2$.

(4) On the event $\mathbf{A}_{2, t_{2}}$, before the arrival of the second particle the process is in $\mathcal{C}_{\bar{\eta}}^{2 U}$. This means that for each time $s$ until that arrival we have $N_{\bar{\Lambda}}\left(\eta_{s}\right) \in\left\{l_{1} l_{2}, l_{1} l_{2}+1\right\}$, and if $N_{\bar{\Lambda}}\left(\eta_{s}\right)$ $=l_{1} l_{2}$, then $\bar{\eta}_{s}=R_{l_{1}, l_{2}}$. This, in turn, implies that the process starting at $\eta_{1}$ during the time interval $[0, \tau)$ can be described as follows:

-During the time intervals in which $N_{\bar{\Lambda}}\left(\eta_{s}\right)=l_{1} l_{2}$, we have one particle moving as a SRW in the presence of a fixed rectangular cluster.

-During the time intervals in which $N_{\Lambda}\left(\eta_{s}\right)=l_{1} l_{2}+1$, the particle can be trapped by the cluster, producing a configuration that inside $\bar{\Lambda}$ falls in $\overline{\mathcal{X}} \backslash \overline{\mathcal{X}}_{2}$. In other words, during this time interval the process behaves like a SRW with a trap at $\partial^{+} R_{l_{1}, l_{2}}$ of escape rate $e^{-U \beta}$. We denote this process by $\left(\hat{\xi}_{s}\right)_{s \geqslant 0}$.

(5) We can now conclude our estimate:

$$
\begin{aligned}
& \int_{e^{U \beta}}^{T} \mathbb{P}_{\eta_{1}}\left(\tau \in d t_{2}, \mathbf{A}_{2, t_{2}} \cap \mathbf{B}_{2, t_{2}}\right) \\
& \quad=\int_{e^{U \beta}}^{T} \mathbb{P}_{\eta_{1}}\left(B_{2, t_{2}} \mid \tau=t_{2}, \mathbf{A}_{2, t_{2}}\right) \mathbb{P}_{\eta_{1}}\left(\tau \in d t_{2}, \mathbf{A}_{2, t_{2}}\right) \\
& \quad \geqslant \int_{e^{U \beta}}^{T} \mathbb{P}_{\eta_{1}}\left(\hat{\xi}_{t_{2}} \in \partial R_{l_{1}, l_{2}}\right) \mathbb{P}_{\eta_{1}}\left(\tau \in d t_{2}, \mathbf{A}_{2, t_{2}}\right) \\
& \geqslant \int_{e^{U \beta}}^{T} \frac{1}{t_{2}} e^{(U-\delta) \beta} \mathbb{P}_{\eta_{1}}\left(\tau \in d t_{2}, \mathbf{A}_{2, t_{2}}\right) \geqslant \frac{1}{T} e^{(U-\delta) \beta} \int_{e^{U \beta}}^{T} \mathbb{P}_{\eta_{1}}\left(\tau \in d t_{2}, \mathbf{A}_{2, t_{2}}\right),
\end{aligned}
$$

where the second inequality uses Proposition 3.13 and the last probability can be estimated as in part (3).

Estimate (ii). 
(1) The lower bound in the case of shrinking transitions can be obtained by forcing the transition to happen within a time $T_{3} e^{-2 \delta_{0} \beta}$ and requiring that during this time no particles enter $\bar{\Lambda}$. In this way the transition can be estimated as in the case of the local Markov chain. More precisely, let $\bar{\eta}=R_{l_{1}, l_{2}}$ and $\bar{\eta}^{\prime}=R_{l_{1}, l_{2}-1}$. For $\eta \in I_{\bar{\eta}} \cap \mathcal{X}_{3}$ we have

$$
P^{R}\left(\eta, I_{\bar{\eta}^{\prime}}\right)=\mathbb{P}_{\eta}\left(\eta_{\tau_{1}} \in I_{\bar{\eta}^{\prime}}\right) \geqslant \mathbb{P}_{\eta, \bar{\eta}}\left(\mathbf{A}_{T_{3} e^{-2 \delta_{0} \beta}} \cap \mathbf{D}_{T_{3} e^{-2 \delta_{0} \beta}} \cap \overline{\mathbf{D}}_{T_{3} e^{-2 \delta_{0} \beta}}\right),
$$

where

$$
\mathbf{A}_{T_{3} e^{-2 \delta_{0} \beta}}=\left\{\eta_{\tau_{1}} \in I_{\bar{\eta}^{\prime}}\right\} \cap\left\{\tau_{1}<T_{3} e^{-2 \delta_{0} \beta}\right\}
$$

$\mathbf{D}_{T_{3} e^{-2 \delta_{0} \beta}}$ is the event where during time $T_{3} e^{-2 \delta_{0} \beta}$ no particles enter $\bar{\Lambda}$;

$\mathbf{D}_{T_{3} e^{-2 \delta_{0} \beta}}$ is the event where during time $T_{3} e^{-2 \delta_{0} \beta}$ no particles are created in $\partial \bar{\Lambda}$.

The last term in the rhs of (7.18) is equal to

$$
\mathrm{P}_{\eta, \bar{\eta}}\left(\overline{\mathbf{A}}_{T_{3} e^{-2 \delta_{0} \beta}} \cap \mathbf{D}_{T_{3} e^{-2 \delta_{0} \beta}} \cap \overline{\mathbf{D}}_{T_{3} e^{-2 \delta_{0} \beta}}\right),
$$

where $\overline{\mathbf{A}}_{T_{3} e^{-2 \delta_{0} \beta}}$ is the analog of $\mathbf{A}_{T_{3} e^{-2 \delta_{0} \beta}}$ for the local Markov chain.

(2) We note that the events $\mathbf{D}_{T_{3} e^{-2 \delta_{0} \beta}}$ and $\overline{\mathbf{A}}_{T_{3} e^{-2 \delta_{0} \beta}} \cap \overline{\mathbf{D}}_{T_{3} e^{-2 \delta_{0} \beta}}$ are independent, since they involve different clocks and marks. Moreover,

$$
\begin{aligned}
& \mathbb{P}_{\eta}\left(\mathbf{D}_{T_{3} e^{-2 \delta_{0} \beta}}\right) \geqslant\left(\frac{\kappa}{\beta}\right)^{\log \beta}
\end{aligned}
$$

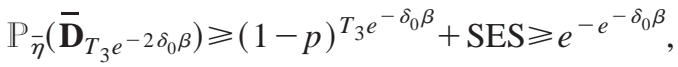

and it is easy to show that

$$
\mathbb{P}_{\bar{\eta}}\left(\overline{\mathbf{A}}_{T_{3} e^{-2 \delta_{0} \beta}} \mid \overline{\mathbf{D}}_{T_{3} e^{-2 \delta_{0} \beta}}\right) \geqslant e^{-\left(2 U-\Delta+3 \delta_{0}\right) \beta} .
$$

Estimate (iii). A similar argument works for the transitions involving the lacunary configurations.

\subsection{Definition of colors and the auxiliary Markov chain}

Recall that $N=\rho\left|\Lambda_{\beta}\right|$ is the total number of particles and that our state space is

$$
\mathcal{N}_{N}=\left\{\eta \in \mathcal{X}: N_{\Lambda_{\beta}}(\eta)=N\right\}, \quad \mathcal{X}=\mathcal{X}_{N}=\{0,1\}^{\bar{\Lambda}} \times\{0,1,2, \ldots, N\}^{\Lambda_{\beta} \backslash \bar{\Lambda}}
$$

7.4.1 Dynamics. We begin by realizing the process $\left(\eta_{t}\right)_{t \geqslant 0}$ in terms of a process in which particles are distinguishable. This means that instead of $\mathcal{X}$ we consider the space $\mathcal{X}_{d}=\Lambda_{\beta}^{N}$ where a configuration is given in terms of the position $\xi(n) \subset \Lambda$ of each particle $n=1, \ldots, N$. Each configuration $\eta \in \mathcal{X}$ corresponds to $N$ ! different configurations in $\mathcal{X}_{d}$. We denote by $n_{t}(x)$ the set of labels of the particles that are at $x$ at time $t$ :

$$
n_{t}(x)=\left\{n=1, \ldots, N: \xi_{t}(n)=x\right\},
$$

so $\eta_{t}(x)=\left|n_{t}(x)\right|$. We note that for any $x \in \bar{\Lambda}$ the set $n_{t}(x)$ contains at most one element.

We can define a stochastic dynamics $\left(\xi_{t}\right)_{t \geqslant 0}$ on $\mathcal{X}_{d}$ corresponding to our stochastic dynamics $\left(\eta_{t}\right)_{t \geqslant 0}$ on $\mathcal{X}$, provided we take the uniform distribution for the initial configuration $\xi_{0}$ corresponding to the initial configuration $\eta_{0}$, i.e., each particle initially gets a label drawn randomly from $\{1, \ldots, N\}$. The rules of this dynamics are straightforward and read as follows: 
For each oriented bond $b \in \bar{\Lambda} * \cup \partial^{*} \bar{\Lambda}^{\text {out }}$, define a sequence of i.i.d. random times $\tau_{b, i}, i$ $\in \mathbb{N}$, exponential with mean 1 , and a sequence of i.i.d. marks $\mu_{b, i}, i \in \mathbb{N}$, uniform on $[0,1]$.

For each oriented bond $b \in\left(\Lambda_{\beta} \backslash \bar{\Lambda}\right) * \cup \partial^{*} \bar{\Lambda}^{\text {in }}$ and each label $1, \ldots, N$, define a sequence of i.i.d. times $\tau(n)_{b, i}, i \in \mathbb{N}$, exponential with mean 1 .

Inside $\Lambda_{\beta} \backslash \bar{\Lambda}$ particle $n$ moves as an IRW with jump times $\tau(n)_{b, i}, i \in \mathbb{N}, b \in\left(\Lambda_{\beta} \backslash \bar{\Lambda}\right)^{*}$.

Inside $\bar{\Lambda}$ particles move with exclusion and with interaction as follows:

When $t=\tau_{b, i}$ for some $b=(x, y) \in \bar{\Lambda}^{*}, i \in \mathbb{N}_{:}$

-If $n_{t}(x)=\{n\}, n_{t}(y)=\varnothing$, then the proposed move is given by $\xi_{t}^{\mathrm{prop}}(n)=y, \xi_{t}^{\mathrm{prop}}(j)$ $=\xi_{t}(j) \forall j \neq n$. This proposed move is accepted if the corresponding mark $\mu_{b, i}$ is less than $e^{-\beta\left[H\left(\eta_{t}^{\text {prop }}\right)-H\left(\eta_{t}\right]_{+}\right.}$, where $\eta_{t}^{\text {prop }}$ and $\eta_{t}$ are the configuration in $\mathcal{X}$ corresponding to $\xi_{t}^{\text {prop }}$, resp., $\xi_{t}$.

-If $n_{t}(x)=\varnothing, n_{t}(y)=\{n\}$, then the proposed move is given by $\xi_{t}^{\text {prop }}(n)=x, \xi_{t}^{\text {prop }}(j)$ $=\xi_{t}(j) \forall j \neq n$. This proposed move is accepted if the corresponding mark $\mu_{b, i}$ is less than $e^{-\beta\left[H\left(\eta_{t}^{\text {prop }}\right)-H\left(\eta_{t}\right]+\right.}$.

-In all other cases there is no move.

When $t=\tau_{b, i}$ for some $b=(x, y) \in \partial^{*} \bar{\Lambda}^{\text {out }}, i \in \mathbb{N}^{\text {: }}$

-If $n_{t}(x)=n$, then $\xi_{t+}(n)=y, \xi_{t^{+}}(j)=\xi_{t}(j) \forall j \neq n$.

-If $n_{t}(x)=\varnothing$, then there is no move.

When $t=\tau(n)_{b, i}$ for some $b=(x, y) \in \partial^{*} \bar{\Lambda}^{\text {in }}, i \in \mathbb{N}$ :

-If $n_{t}(x)=n$ and $n_{t}(y)=\varnothing$, then $\xi_{t+}(n)=y, \xi_{t+}(j)=\xi_{t}(j) \forall j \neq n$.

Otherwise there is no move.

7.4.2. Coloring of particles. Next we assign a color to each particle. This color depends on time and will later be used to distinguish between particles arriving from the gas and particles returning to $\bar{\Lambda}_{0}$ after leaving it.

Definition 7.24: (a) Every particle in $\xi_{t} \cap \bar{\Lambda}$ is WHITE at time $t$.

(b) Every particle in $\xi_{t} \cap \Lambda_{\beta} \backslash \Lambda_{l_{-}}$is GREEN at time $t$.

(c) Particles in $\Lambda_{l_{-}} \backslash \bar{\Lambda}$ are GREEN or RED depending on their past in the following way. For $n \in n_{t}(x)$, let

$$
\theta=\theta(n, t)=\max \left\{0 \leqslant s<t: \xi_{s}(n) \notin \Lambda_{l_{-}} \backslash \bar{\Lambda}\right\} .
$$

If $\xi_{\theta}(n) \in \bar{\Lambda}$, then $n$ is a RED particle at time t. If $\xi_{\theta}(n) \in \Lambda_{\beta} \backslash \Lambda_{l_{-}}$, then $n$ is a GREEN particle at time $t$.

It is easy to see that if the process starts from a configuration $\eta \in \mathcal{X}_{3}$, then the time $\theta$ is well-defined for each particle $\xi_{t}(n) \in \Lambda_{l_{-}} \backslash \bar{\Lambda}$.

The colors at different times are obviously correlated, since when a particle leaves $\bar{\Lambda}$ it become red. Green particles become white when they enter $\bar{\Lambda}$. Red particles become white or green when they leave $\Lambda_{l} \backslash \bar{\Lambda}$.

In order to control the behavior of green and red particles, we separate their effects by introducing an auxiliary Markov chain $\left(\widetilde{\eta}_{t}\right)_{t \geqslant 0}$ in which the arrival in $\bar{\Lambda}$ of green particles is simulated by a process of creation at $\partial^{-} \bar{\Lambda}$ with a rate of order $e^{-\beta \Delta}$ and a process of annihilation at $\partial^{+} \bar{\Lambda}$ with rate 1 .

7.4.3. Auxiliary Markov chain. We define the dynamics $\left(\widetilde{\eta}_{t}\right)_{t \geqslant 0}$ by means of a process $\left(\widetilde{\xi}_{t}\right)_{t \geqslant 0}$ of distinguishable particles. Fix a parameter 


$$
p=\frac{1}{N T_{3}} e^{-\gamma_{0} \beta} \text { with } T_{3}=e^{\Delta \beta},
$$

where $\gamma_{0}$ is small and will be chosen later. Let

$$
\tilde{\mathcal{X}}_{d}=\left(\Lambda_{l_{-}} \cup\{g\}\right)^{N} .
$$

This means that each particle in the auxiliary dynamics can be in a site of $\Lambda_{l_{-}}$or in a state called $g$ (for green).

We can use the same ingredients as for the Markov chain $\left(\xi_{t}\right)_{t \geqslant 0}$ for the bonds in $\Lambda_{l_{-}}^{*} \cup \partial^{*} \Lambda_{l_{-}}^{\text {out }}$, but we need to add for each bond $b \in \partial^{*} \bar{\Lambda}^{\text {in }}$ a sequence of i.i.d. marks $\mu_{b, i}^{c}, i$ $\in \mathbb{N}$, uniform on $[0,1]$. The stochastic dynamics $\left(\widetilde{\xi}_{t}\right)_{t \geqslant 0}$ can be realized exactly as $\left(\xi_{t}\right)_{t \geqslant 0}$ for each bond $b \notin \partial^{*} \bar{\Lambda}^{\text {in }}$. If $t=\tau(n)_{b, i}$ for some $b=(x, y) \in \partial^{*} \bar{\Lambda}^{\text {in }}, i \in \mathbb{N}$, then we consider an additional move of creation of a particle at site $y$. The details are again straightforward and read as follows:

Inside $\Lambda_{l_{-}} \backslash \bar{\Lambda}$ particle $n$ moves as an IRW with jump times $\tau(n)_{b, i}$.

Inside $\bar{\Lambda}$ particles move with exclusion and with interaction as for $\left(\xi_{t}\right)_{t \geqslant 0}$.

When $t=\tau_{b, i}$ for some $b=(x, y) \in \partial^{*} \bar{\Lambda}^{\text {out }}, i \in \mathbb{N}$ :

-If $n_{t}(x)=\{n\}$, then $\widetilde{\xi}_{t+}(n)=y, \widetilde{\xi}_{t+}(j)=\widetilde{\xi}_{t}(j) \forall j \neq n$.

-If $n_{t}(x)=\varnothing$, then there is no move.

When $t=\tau(n)_{b, i}$ for some $b=(x, y) \in \partial^{*} \bar{\Lambda}^{\text {in }}, i \in \mathbb{N}$ :

-If $n_{t}(x)=n$ and $n_{t}(y)=\varnothing$, then $\widetilde{\xi}_{t+}(n)=y$.

-If $\widetilde{\xi}_{t}(n)=g$ and $n_{t}(y)=\varnothing$, then $\widetilde{\xi}_{t+}(n)=y$ when $\mu_{b, i}^{c}<p$, otherwise there is no move.

When $t=\tau(n)_{b, i}$ for some $b=(x, y) \in \partial^{*} \Lambda_{l_{-}}^{\text {out }}, i \in \mathbb{N}$ :

-If $n_{t}(x)=n$, then $\widetilde{\xi}_{t^{+}}(n)=g$, otherwise there is no move.

We will consider the discrete-time Markov chains corresponding to $\left(\xi_{t}\right)_{t \geqslant 0}$ and $\left(\widetilde{\xi}_{t}\right)_{t \geqslant 0}$ by observing these processes when a clock rings in $\Lambda_{\beta}$. From now on we will consider only these discrete versions. By Proposition 2.2 we know that the control of the discrete-time Markov chain enables us to control the continuous-time Markov chain.

Now that the process $\left(\widetilde{\xi}_{t}\right)_{t \geqslant 0}$ is defined, the definition of $\widetilde{\eta}_{t}$ in terms of $\widetilde{\xi}_{t}$ is given exactly as $\eta_{t}$ in terms of $\xi_{t}$. The state space $\tilde{\mathcal{X}}$ is

$$
\tilde{\mathcal{X}}=\tilde{\mathcal{X}}_{N}=\{0,1\}^{\bar{\Lambda}} \times\{0,1,2, \ldots, N\}^{\left(\Lambda_{l_{-}} \backslash \bar{\Lambda}\right) \cup\{g\}} .
$$

7.4.4. Coupling. We can define a coupling between the Markov chains $\left(\eta_{t}\right)_{t \geqslant 0}$ and $\left(\tilde{\eta}_{t}\right)_{t \geqslant 0}$ by using the same clocks and marks for common bonds. Two events will be important.

$\mathbf{B}_{\eta}\left(t_{1}, \ldots, t_{k} ; b_{1}, \ldots, b_{k} ; n_{1}, \ldots, n_{k} ; t\right)$ is the event for $\left(\eta_{t}\right)_{t \geqslant 0}$ where during the time interval $[0$, $t]$ green particles enter $\bar{\Lambda}$ through the bonds $b_{1}, \ldots, b_{k} \in \partial^{*} \bar{\Lambda}^{\text {in }}$ at times $t_{1}, \ldots, t_{k}$ and their labels are $n_{1}, \ldots, n_{k}$.

$\mathbf{B}_{\tilde{\eta}}\left(t_{1}, \ldots, t_{k} ; b_{1}, \ldots, b_{k} ; n_{1}, \ldots, n_{k} ; t\right)$ is the event for $\left(\widetilde{\eta}_{t}\right)_{t \geqslant 0}$ where during the time interval $[0$, $t]$ particles are created through the bonds $b_{1}, \ldots, b_{k} \in \partial^{*} \bar{\Lambda}^{\text {in }}$ at times $t_{1}, \ldots, t_{k}$ and their labels are $n_{1}, \ldots, n_{k}$.

Let us take two initial configurations $\eta_{0}$ and $\widetilde{\eta}_{0}$, such that $\eta_{0}(x)=0$ for all $x \in \Lambda_{l_{-}} \backslash \bar{\Lambda}$ and $\eta_{0}(x)=\widetilde{\eta}_{0}(x)$ for all $x \in \Lambda_{l_{-}}$. If both

$$
\begin{aligned}
& \mathbf{B}_{\eta}\left(t_{1}, \ldots, t_{k} ; b_{1}, \ldots, b_{k} ; n_{1}, \ldots, n_{k} ; t\right) \\
& \mathbf{B}_{\tilde{\eta}}\left(t_{1}, \ldots, t_{k} ; b_{1}, \ldots, b_{k} ; n_{1}, \ldots, n_{k} ; t\right)
\end{aligned}
$$


occur, then we have that for all $s \in[0, t]$ the red and the white particles of $\eta_{s}$ coincide with the particles of $\widetilde{\eta}_{s}$, i.e., if

$$
n_{s}^{w, r}=\{n=1, \ldots, N: n \text { is a white or red particle at time } s\},
$$

then

$$
\begin{aligned}
\mathbb{P}_{\xi, \tilde{\xi}}\left(\forall s \in[0, t]:\left\{\xi_{s}(n)=\tilde{\xi}_{s}(n) \quad \forall n \in n_{s}^{w, r}\right\} \cap\left\{\tilde{\xi}_{s}=g \quad \forall n \notin n_{s}^{w, r}\right\} \mid\right. \\
\\
\quad \mathbf{B}_{\eta}\left(t_{1}, \ldots, t_{k} ; b_{1}, \ldots, b_{k} ; n_{1}, \ldots, n_{k} ; t\right) \cap \\
\left.\mathbf{B}_{\tilde{\eta}}\left(t_{1}, \ldots, t_{k} ; b_{1}, \ldots, b_{k} ; n_{1}, \ldots, n_{k} ; t\right)\right)=1 .
\end{aligned}
$$

In a trivial way we can define a coupling between $\left(\widetilde{\eta}_{t}\right)_{t \geqslant 0}$ and the local Markov chain $\left(\bar{\eta}_{t}\right)_{t \geqslant 0}$ defined in Sec. 4.1 by using the same clocks and marks for bonds in $\Lambda_{0}^{*} \cup \partial^{*} \bar{\Lambda}^{\text {out }}$ and the same clocks and creation marks for bonds in $b \in \partial^{*} \bar{\Lambda}^{\text {in }}$. In particular, also for the Markov chain $\left(\bar{\eta}_{t}\right)_{t \geqslant 0}$ we can realize the creation at the boundary of $\bar{\Lambda}$ by using $N$ clocks and the same rate $p$ in (7.26) used for $\left(\widetilde{\eta}_{t}\right)_{t \geqslant 0}$.

Remark:

(1) If $\gamma_{0}>0$ in the definition of $p$ is such that $l_{c}\left(\Delta+\gamma_{0}\right)=l_{c}(\Delta)$, then we can apply the remark given at the end of Sec. 5. If $\Delta \in(U, 2 U)$ is such that $U /(2 U-\Delta)$ is not integer, then there exists such a $\gamma_{0}$.

(2) It is obvious that if no red particles enter $\bar{\Lambda}$ during the time interval $[0, t]$, then $\left.\widetilde{\eta}_{s}\right|_{\bar{\Lambda}}$ $=\bar{\eta}_{s} \mid \bar{\Lambda}$ for all $s \in[0, t]$.

(3) As in the case of the full Markov chain $\left(\eta_{t}\right)_{t \geqslant 0}$, we can define recurrence sets $\widetilde{\mathcal{X}}_{1}, \tilde{\mathcal{X}}_{2}, \tilde{\mathcal{X}}_{3}$ and we can prove also for $\left(\widetilde{\eta}_{t}\right)_{t \geqslant 0}$ the recurrence properties to these sets. In the same way we can define the reduced Markov chains $\left(\tilde{\eta}_{t}^{R}\right)_{t \geqslant 0}$ and $\left(\bar{\eta}_{t}^{R}\right)_{t \geqslant 0}$ (recall Definition 7.3). The only difference is that for $\left(\widetilde{\eta}_{t}\right)_{t \geqslant 0}$ we can only prove the recurrence to $\tilde{\mathcal{X}}_{3}$ in a time $(p N)^{-1} e^{\delta_{1} \beta}$ $=T_{3} e^{\left(\gamma_{0}+\delta_{1}\right) \beta}$ rather than in a time $T_{3} e^{\delta_{1} \beta}$ as in Proposition 6.2.

\subsection{Upper bounds}

The key to Proposition 7.9 (iv) is the following [recall (7.13)].

Proposition 7.32: There exist $\delta=\delta(\gamma)$, satisfying $\lim _{\gamma \downarrow 0} \delta(\gamma)=0$, and $\beta_{0}>0$ such that, for all $\beta>\beta_{0}$ and $\bar{\eta} \in \overline{\mathcal{X}}_{3}$,

$$
\max _{\eta \in I_{\bar{\eta}} \cap \mathcal{X}_{3}} \mathbb{P}_{\eta}\left(\tau_{C_{\bar{\eta}}^{c}}<T_{3} e^{\delta_{1} \beta}\right) \leqslant e^{-[r(\bar{\eta})-\delta] \beta},
$$

where $\delta_{1}$ is the parameter appearing in Proposition 6.2, and

$$
\mathcal{C}_{\bar{\eta}}=\left\{\eta^{\prime} \in \mathcal{X}:\left.\eta^{\prime}\right|_{\bar{\Lambda}} \in \overline{\mathcal{C}}_{\bar{\eta}}\right\}
$$

with

$$
\overline{\mathcal{C}}_{\bar{\eta}}=\left\{\bar{\eta}^{\prime} \in \overline{\mathcal{X}}: \bar{H}\left(\bar{\eta}^{\prime}, \bar{\eta}\right)-\bar{H}(\bar{\eta})<r(\bar{\eta})+\Delta\right\} .
$$

Proof of Proposition 7.9 (iv): Let $\mathbf{M}_{\eta}$ denote the event that the transition $\eta^{R}: \eta \rightarrow \eta^{\prime}$ occurs within time $T_{3} e^{\delta_{1} \beta}$. By the recurrence property of the set $\mathcal{X}_{3}$ in Proposition 6.2, we have 


$$
\mathrm{P}_{\eta}\left(\mathbf{M}_{\eta}^{c}\right) \leqslant \mathrm{SES}
$$

Hence

$$
\begin{gathered}
\max _{\eta \in I_{\bar{\eta}} \bar{\eta}^{\prime} \in \overline{\mathcal{X}}_{3}: \bar{\eta}^{\prime} \notin \mathcal{E}_{3}(\bar{\eta})} \mathrm{P}_{\eta}\left(\eta^{R}: \eta \rightarrow I_{\bar{\eta}^{\prime}}\right) \\
\leqslant \max _{\eta \in I_{\bar{\eta}}} \max _{\bar{\eta}^{\prime} \in \overline{\mathcal{X}}_{3}: \bar{\eta}^{\prime} \notin \mathcal{E}_{3}(\bar{\eta})} \mathbb{P}_{\eta}\left(\eta^{R}: \eta \rightarrow I_{\bar{\eta}^{\prime}} \cap \mathbf{M}_{\eta}\right)+\mathrm{SES} \\
\quad \max _{\eta \in I_{\bar{\eta}}} \mathbb{P}_{\eta}\left(\tau_{C_{\bar{\eta}}^{c}<T_{3} e^{\delta_{1} \beta}}\right)+\mathrm{SES} .
\end{gathered}
$$

In fact, if $\bar{\eta}^{\prime} \in \overline{\mathcal{X}}_{3}$ and $\bar{\eta}^{\prime} \notin \mathcal{E}_{3}(\bar{\eta})$, then $\bar{\eta}^{\prime} \notin \overline{\mathcal{C}}_{\bar{\eta}}$ by Lemma 5.34. QED

Proof of Proposition 7.32: Abbreviate for $\left(\widetilde{\eta}_{t}\right)_{t \geqslant 0}$ :

$$
\mathbf{A}_{\eta}=\left\{\tau_{\mathcal{C}_{\bar{\eta}}^{c}}<T_{3} e^{\delta_{1} \beta}\right\}
$$

and analogously for $\left(\widetilde{\eta}_{t}\right)_{t \geqslant 0}$ :

$$
\mathbf{A}_{\bar{\eta}}=\left\{\tau_{\widetilde{\mathcal{C}}_{\bar{\eta}}^{c}}<T_{3} e^{\delta_{1} \beta}\right\}
$$

where

$$
\tilde{\mathcal{C}}_{\bar{\eta}}=\left\{\widetilde{\eta}^{\prime} \in \tilde{\mathcal{X}}:\left.\widetilde{\eta}^{\prime}\right|_{\bar{\Lambda}} \in \overline{\mathcal{C}}_{\bar{\eta}}\right\}
$$

Propositions 7.41 and 7.43 below imply Proposition 7.32.

Proposition 7.41: There exist $\delta=\delta(\gamma)$, satisfying $\lim _{\gamma \downarrow 0} \delta(\gamma)=0$, and $\beta_{0}>0$ such that, for all $\beta>\beta_{0}$ and $\eta^{\prime} \in \mathcal{X}_{3}$,

$$
\mathbb{P}_{\eta^{\prime}}\left(\mathbf{A}_{\eta}\right) \leqslant \mathbb{P}_{\eta^{\prime}}\left(\mathbf{A}_{\tilde{\eta}}\right) e^{\delta \beta} .
$$

Proposition 7.43: There exist $\delta=\delta(\gamma)$, satisfying $\lim _{\gamma \downarrow 0} d(\gamma)=0$, and $\beta_{0}>0$ such that, for all $\beta>\beta_{0}$ and $\eta^{\prime} \in \mathcal{X}_{3}$,

$$
\mathrm{P}_{\eta^{\prime}}\left(\mathbf{A}_{\tilde{\eta}}\right) \leqslant e^{-r(\bar{\eta}) \beta+\delta \beta} .
$$

QED

The proof of Propositions 7.41 and 7.43 is given in Sec. 7.6, resp. Sec. 7.7.

\subsection{Control of green particles}

We henceforth suppress the lower index from $\mathrm{P}$ when the initial configuration is obvious.

Proof of Proposition 7.41: The problem is to compare the effect of green particles versus the creation mechanism in the auxiliary dynamics.

(1) Fix $k \in \mathbb{N}, t_{1}, \ldots, t_{k} \geqslant 0, b_{1}, \ldots, b_{k} \in \partial^{*} \bar{\Lambda}^{\text {in }}$, and abbreviate

$$
\begin{aligned}
& \mathbf{B}_{\eta}=\mathbf{B}_{\eta}\left(t_{1}, \ldots, t_{k} ; b_{1}, \ldots, b_{k} ; n_{1}, \ldots, n_{k} ; T_{3} e^{\delta_{1} \beta}\right), \\
& \mathbf{B}_{\tilde{\eta}}=\mathbf{B}_{\tilde{\eta}}\left(t_{1}, \ldots, t_{k} ; b_{1}, \ldots, b_{k} ; n_{1}, \ldots, n_{k} ; T_{3} e^{\delta_{1} \beta}\right) .
\end{aligned}
$$

We begin by proving the following.

Lemma 7.46: There exist $\delta^{\prime}=\delta^{\prime}(\gamma)$, satisfying $\lim _{\gamma \downarrow 0} \delta^{\prime}(\gamma)=0$, and $\beta_{0}>0$ such that, for all $\beta>\beta_{0}$ and $\eta^{\prime} \in \mathcal{X}_{3}$,

$$
\mathbb{P}\left(\mathbf{A}_{\tilde{\eta}} \cap \mathbf{B}_{\tilde{\eta}}\right)\left(\frac{e^{-\left(\Delta-\delta^{\prime}\right) \beta}}{N}\right)^{k} \geqslant \mathbb{P}\left(\mathbf{A}_{\tilde{\eta}} \cap \mathbf{B}_{\eta} \cap \mathbf{B}_{\tilde{\eta}}\right) \geqslant \mathbb{P}\left(\mathbf{A}_{\eta} \cap \mathbf{B}_{\eta} \cap \mathbf{B}_{\tilde{\eta}}\right) \geqslant \mathbb{P}\left(\mathbf{A}_{\eta} \cap \mathbf{B}_{\eta}\right)\left(\frac{e^{-\left(\Delta+\delta^{\prime}\right) \beta}}{N}\right)^{k}
$$


Proof: (1) Third inequality: Let $\mathbf{C}_{\tilde{\eta}}$ be the event that the number of rings by clocks on the bonds in $\partial^{*} \bar{\Lambda}^{\text {in }}$ within time $T_{3} e^{\delta_{1} \beta}$ is less than $N T_{3} e^{2 \delta_{1} \beta}$. Then

$$
\mathbb{P}\left(\mathbf{A}_{\eta} \cap \mathbf{B}_{\eta} \cap \mathbf{B}_{\tilde{\eta}}\right) \geqslant \mathbb{P}\left(\mathbf{A}_{\eta} \cap \mathbf{B}_{\eta} \cap \mathbf{B}_{\tilde{\eta}} \cap \mathbf{C}_{\tilde{\eta}}\right)
$$

and

$$
\mathbb{P}\left(\mathbf{C}_{\tilde{\eta}}\right)=1-\text { SES } .
$$

We have

$$
\begin{aligned}
\mathbb{P}\left(\mathbf{A}_{\eta} \cap \mathbf{B}_{\eta} \cap \mathbf{B}_{\tilde{\eta}} \cap \mathbf{C}_{\tilde{\eta}}\right) & \geqslant \mathbb{P}\left(\mathbf{B}_{\tilde{\eta}} \mid \mathbf{A}_{\eta} \cap \mathbf{B}_{\eta} \cap \mathbf{C}_{\tilde{\eta}}\right) \mathbb{P}\left(\mathbf{A}_{\eta} \cap \mathbf{B}_{\eta} \cap \mathbf{C}_{\tilde{\eta}}\right) \\
& \geqslant p^{k}(1-p)^{N T_{3} e^{2 \delta_{1} \beta}} \mathbb{P}\left(\mathbf{A}_{\eta} \cap \mathbf{B}_{\eta} \cap \mathbf{C}_{\tilde{\eta}}\right) .
\end{aligned}
$$

If $\gamma_{0}$ in the definition of $p$ in (7.26) satisfies $\gamma_{0}>2 \delta_{1}$, then

$$
\mathrm{P}\left(\mathbf{A}_{\eta} \cap \mathbf{B}_{\eta} \cap \mathbf{B}_{\tilde{\eta}} \cap \mathbf{C}_{\tilde{\eta}}\right) \geqslant p^{k} e^{-\delta_{1} \beta} \mathbb{P}\left(\mathbf{A}_{\eta} \cap \mathbf{B}_{\eta}\right)+\mathrm{SES} \geqslant p^{k} e^{-2 \delta_{1} \beta} \mathbb{P}\left(\mathbf{A}_{\eta} \cap \mathbf{B}_{\eta}\right) .
$$

(2) Second inequality: This immediately follows from the coupling between $\left(\eta_{t}\right)_{t \geqslant 0}$ and $\left(\widetilde{\eta}_{t}\right)_{t \geqslant 0}$.

(3) First inequality: This follows from Proposition 3.31. The argument runs as follows.

(3i) If $t_{1} \leqslant e^{(\Delta-2 \gamma) \beta}$, then

$$
\mathbb{P}\left(\mathbf{A}_{\tilde{\eta}} \cap \mathbf{B}_{\tilde{\eta}} \cap \mathbf{B}_{\eta}\right) \leqslant \mathbb{P}\left(\mathbf{A}_{\tilde{\eta}} \cap \mathbf{B}_{\bar{\eta}} \cap \mathbf{C}_{\eta}\right)=\operatorname{P}\left(\mathbf{A}_{\tilde{\eta}} \cap \mathbf{B}_{\tilde{\eta}}\right) \mathrm{P}\left(\mathbf{C}_{\eta}\right)
$$

with

$$
\mathbf{C}_{\eta}\left\{\exists \text { particle in } \eta \cap \Lambda_{l_{-}}^{c} \text { hitting } \bar{\Lambda} \text { within time } t_{1}\right\} \text {. }
$$

By Proposition 3.25, $\mathrm{P}\left(\mathbf{C}_{\eta}\right)<\mathrm{SES}$.

(3ii) If $t_{1}>e^{(\Delta-2 \gamma) \beta}$ and if there exist $1 \leqslant i<j \leqslant k$ with $n_{i}=n_{j}$ and $t_{j}-t_{i} \leqslant e^{(\Delta-2 \gamma) \beta}$, then

$$
\mathbb{P}\left(\mathbf{A}_{\tilde{\eta}} \cap \mathbf{B}_{\tilde{\eta}} \cap \mathbf{B}_{\eta}\right) \leqslant \mathrm{P}\left(\mathbf{A}_{\tilde{\eta}} \cap \mathbf{B}_{\tilde{\eta}}\right) \mathrm{P}\left(\mathbf{C}_{\eta}\right)
$$

with

$$
\mathbf{C}_{\eta}=\left\{\xi_{t_{i}}\left(n_{i}\right)=x_{i}, \xi_{t}\left(n_{i}\right) \in \Lambda_{l_{-}}^{c} \quad \exists t \in\left(t_{i}, t_{j}\right), \xi_{t_{j}}\left(n_{i}\right)=x_{j}\right\} .
$$

Again by Proposition 3.25, $\mathrm{P}\left(\mathbf{C}_{\eta}\right)<$ SES.

(3iii) If $t_{1}>e^{(\Delta-2 \gamma) \beta}$ and if $t_{j}-t_{i}>e^{(\Delta-2 \gamma) \beta}$ for all $1 \leqslant i<j \leqslant k$ such that $n_{i}=n_{j}$, then

$$
\mathbb{P}\left(\mathbf{A}_{\tilde{\eta}} \cap \mathbf{B}_{\tilde{\eta}} \cap \mathbf{B}_{\eta}\right) \leqslant \mathbb{P}\left(\mathbf{A}_{\tilde{\eta}} \cap \mathbf{B}_{\tilde{\eta}} \cap \mathbf{C}_{\eta}\right)-\operatorname{SES}=\mathbb{P}\left(\mathbf{A}_{\tilde{\eta}} \cap \mathbf{B}_{\tilde{\eta}}\right) \mathbb{P}\left(\mathbf{C}_{\eta}\right)-\operatorname{SES}
$$

with

$$
\mathbf{C}_{\eta}=\left\{\begin{array}{c}
\xi_{t_{1}}\left(n_{1}\right)=x_{1}, \xi_{t}\left(n_{1}\right) \notin \bar{\Lambda} \quad \forall t \in\left[t_{1}-e^{(\Delta-2 \gamma) \beta}, t_{1}\right] \\
\ldots \\
\xi_{t_{k}}\left(n_{k}\right)=x_{k}, \xi_{t}\left(n_{k}\right) \notin \bar{\Lambda} \quad \forall t \in\left[t_{k}-e^{(\Delta-2 \gamma) \beta}, t_{k}\right]
\end{array}\right\} .
$$

By using the independence of the random walks outside $\bar{\Lambda}$ and the fact that if $n_{i}=n_{j}$ then $t_{j}$ $-t_{i}>e^{(\Delta-2 \gamma) \beta}$, we have 


$$
\mathbb{P}\left(\mathbf{C}_{\eta}\right)=\prod_{i=1}^{k} \mathbb{P}\left(\xi_{t_{i}}\left(n_{i}\right)=x_{i}, \xi_{t}\left(n_{i}\right) \notin \bar{\Lambda} \quad \forall t \in\left[t_{i}-e^{(\Delta-2 \gamma) \beta}, t_{i}\right]\right) .
$$

By now using Proposition 3.31, we arrive at

$$
\mathbb{P}\left(\mathbf{C}_{\eta}\right) \leqslant\left(\frac{e^{-\left(\Delta-\delta^{\prime}\right) \beta}}{N}\right)^{k} .
$$

The factor $1 / N^{k}$ comes from the fact that Proposition 3.31 gives an estimate for a process with indistinguishable particles and the probability that a given particle has a given label is $1 / N$. QED

(2) Continuing the proof of Proposition 7.41, we write out

$$
\begin{aligned}
\mathbb{P}\left(\mathbf{A}_{\eta}\right)= & \sum_{k=0}^{\infty} \sum_{b_{1}, \ldots, b_{k}} \sum_{n_{1}, \ldots, n_{k}} \sum_{t_{1}=0}^{T_{3} e^{\delta_{1} \beta}} \cdots \sum_{t_{k}=0}^{T_{3} e^{\delta_{1} \beta}} \\
& \times \mathbb{P}\left(\mathbf{A}_{\eta} \cap \mathbf{B}_{\eta}\left(t_{1}, \ldots, t_{k} ; b_{1}, \ldots, b_{k} ; n_{1}, \ldots, n_{k} ; T_{3} e^{\delta_{1} \beta}\right)\right),
\end{aligned}
$$

and a similar expansion for $\mathrm{P}\left(\mathbf{A}_{\tilde{\eta}}\right)$. By Lemma 7.46 we have

$$
\mathbb{P}\left(\mathbf{A}_{\eta} \cap \mathbf{B}_{\eta}\right) \leqslant \mathbb{P}\left(\mathbf{A}_{\tilde{\eta}} \cap \mathbf{B}_{\tilde{\eta}}\right) e^{2 \delta^{\prime} k \beta},
$$

and so we obtain

$$
\mathbb{P}\left(\mathbf{A}_{\eta}\right) \leqslant \sum_{k=0}^{\infty} e^{2 \delta^{\prime} k \beta} \mathbb{P}\left(\mathbf{A}_{\tilde{\eta}} \cap\{\nu=k\}\right)=\sum_{k=0}^{\infty} e^{2 \delta^{\prime} k \beta} \mathrm{P}\left(\mathbf{A}_{\tilde{\eta}} \mid\{\nu=k\}\right) \mathbb{P}(\{\nu=k\}),
$$

where $\nu$ is the number of particles created by the process $\left(\widetilde{\eta}_{t}\right)_{t \geqslant 0}$ during the transition we are considering. It is easy to see that there exists a $K>0$ independent of $\beta$ such that

$$
\mathrm{P}(\nu=k) \leqslant e^{-K k \beta} \quad \forall k \in \mathbb{N}_{0}
$$

for $\beta$ sufficiently large.

(3) Let $a=4 \delta^{\prime} / K, f=\mathbb{P}\left(\mathbf{A}_{\eta} \mid\{\nu=k\}\right) \mathrm{P}(\{\nu=k\})^{1-a}$ and $g=e^{2 \delta^{\prime} k \beta} \mathrm{P}(\{\nu=k\})^{a}$. By applying Hölder's inequality with $p=1 /(1-a)$ and $q=1 / a$, we obtain

$$
\begin{aligned}
\mathbb{P}\left(\mathbf{A}_{\eta}\right) & \leqslant\left(\sum_{k=0}^{\infty} f^{p}\right)^{1 / p}\left(\sum_{k=0}^{\infty} g^{q}\right)^{1 / q} \\
& =\left(\sum_{k=0}^{\infty} \mathbb{P}\left(\mathbf{A}_{\tilde{\eta}} \mid\{\nu=k\}\right)^{1 /(1-a)} \mathbb{P}(\{\nu=k\})\right)^{1-a}\left(\sum_{k=0}^{\infty} e^{2 \delta^{\prime} k \beta / a} \mathbb{P}(\{\nu=k\})\right)^{a} \\
& \leqslant C\left(a, K, \delta^{\prime}\right) \mathbb{P}\left(\mathbf{A}_{\tilde{\eta}}\right)^{1-a},
\end{aligned}
$$

where the last inequality uses (7.63). But a rough estimate gives

$$
\mathrm{P}\left(\mathbf{A}_{\tilde{\eta}}\right) \geqslant e^{-\left(\Delta l_{2}-\delta^{\prime \prime}\right) \beta}
$$

for an arbitrary $\delta^{\prime \prime}>0$, obtained by creating $l_{2}$ particles and bringing them to the cluster in a time of order one. Hence we get

$$
\mathbb{P}\left(\mathbf{A}_{\eta}\right) \leqslant \mathbb{P}\left(\mathbf{A}_{\tilde{\eta}}\right) C\left(a, K, \delta^{\prime}\right) e^{a\left(\Delta+\delta^{\prime \prime}\right) l_{2} \beta},
$$

which completes the proof of Proposition 7.41 after setting $\delta=a\left(\Delta+\delta^{\prime \prime}\right) l_{2}$. QED 


\subsection{Control of red particles}

We now come to the hardest part of the argument, because we have to control the effect of red particles.

Proof of Proposition 7.43. In order to control every possible mechanism of exit from the set $\tilde{\mathcal{C}}_{\tilde{\eta}}$, we analyze the final exit move. More precisely, we consider a partition of the set $\widetilde{\mathcal{C}}_{\tilde{\eta}}$ (essentially in terms of its intersection with the sets $\overline{\mathcal{X}}_{1}, \overline{\mathcal{X}}_{2}, \overline{\mathcal{X}}_{3}$ ). and we compute the cost (in term of the difference of energy) of the final exiting move starting from an element of this partition. This final move can be obtained by the arrival of a red particle inside $\bar{\Lambda}$ or by a move inside $\bar{\Lambda}_{0}$. We estimate the probability of the event $\mathbf{A}_{\tilde{\eta}}$ in terms of the probability of this final move. As far as the probability of the arrival of a red particle is concerned, we show that red particles essentially behave as IRW's.

We divide this proof into several lemmas, the proof of which is deferred to Sec. 7.8. We first consider the case $\bar{\eta} \in \overline{\mathcal{X}}_{3} \backslash \mathcal{L}$.

(1) We recall from (5.16) that for a configuration $\bar{\eta}^{\prime}$ in $\bar{\Lambda}$ with contour $\gamma^{\prime}=\gamma^{\prime}\left(\bar{\eta}^{\prime}\right)$ :

$$
\bar{H}\left(\bar{\eta}^{\prime}\right)=(-2 U+\Delta) n_{0}^{\prime}+\frac{U}{2}\left|\gamma^{\prime}\right|+\Delta \eta^{\prime},
$$

where $n_{0}^{\prime}$ is the total number of particles in $\bar{\Lambda}_{0}, n^{\prime}$ is the total number of particles in the ring $\bar{\Lambda} \backslash \bar{\Lambda}_{0}$. We will use this expression to classify the configurations in $\overline{\mathcal{C}}_{\bar{\eta}}$ in terms of the sets $\overline{\mathcal{X}}_{1}, \overline{\mathcal{X}}_{2}$ and in terms of the cost to exit from $\overline{\mathcal{C}}_{\bar{\eta}}$ in one move, defined by

$$
\operatorname{EC}\left(\bar{\eta}^{\prime}\right)=\min _{\bar{\eta}^{\prime \prime} \notin \overline{\mathcal{C}}_{\bar{\eta}}: q\left(\bar{\eta}^{\prime}, \bar{\eta}^{\prime \prime}\right)>0} \bar{H}\left(\bar{\eta}^{\prime \prime}\right)-\bar{H}\left(\bar{\eta}^{\prime}\right) \quad\left(\bar{\eta}^{\prime} \in \overline{\mathcal{C}}^{\prime} \bar{\eta}\right),
$$

where we put $\operatorname{EC}\left(\bar{\eta}^{\prime}\right)=\infty$ if $q\left(\bar{\eta}^{\prime}, \bar{\eta}^{\prime \prime}\right)=0$ for all $\bar{\eta}^{\prime \prime} \notin \overline{\mathcal{C}}_{\bar{\eta}}$.

Note that, by the definition of the set $\overline{\mathcal{C}}_{\bar{\eta}}$ in (7.35), the exit cost is strictly positive for all $\bar{\eta}^{\prime} \in \overline{\mathcal{C}}_{\bar{\eta}}$ and thus $\operatorname{EC}\left(\bar{\eta}^{\prime}\right)$ assumes the values $U, \Delta, 2 U, 3 U$.

Lemma 7.69: For all $\bar{\eta} \in \overline{\mathcal{X}}_{3} \backslash \mathcal{L}$ the following hold:

(i) If $\bar{\eta}^{\prime} \in \overline{\mathcal{C}}_{\bar{\eta}}$, then its contour is larger than or equal to that of $\bar{\eta}$ (i.e., $\left|\gamma^{\prime}\right| \geqslant|\gamma|$ ) and its number of internal particles $n_{0}^{\prime}$ belongs to the interval $\left[n_{0}-l_{1}+2, n_{0}+1\right]$, where $n_{0}$ is the number of internal particles of $\bar{\eta}$ and $l_{1}$ is the minimal side length of $\bar{\eta}$.

(ii) Let $\bar{\eta}^{\prime} \in \overline{\mathcal{C}}_{\bar{\eta}},\left|\gamma^{\prime}\right|=|\gamma|$ and $n^{\prime}=0$. Then

$$
n_{0}^{\prime}=n_{0}-l^{\prime} \quad \text { for some } l^{\prime}=0,1, \ldots, l_{1}-2 \text {. }
$$

Moreover, $\bar{\eta}^{\prime} \in \overline{\mathcal{X}}_{2}$ and

$$
\begin{array}{ll}
\mathrm{EC}\left(\bar{\eta}^{\prime}\right)=3 U & \text { if } \quad l^{\prime}=0,1, \ldots, l_{1}-3, \\
\mathrm{EC}\left(\bar{\eta}^{\prime}\right)=2 U & \text { if } \quad l^{\prime}=l_{1}-2 .
\end{array}
$$

(iii) Let $\bar{\eta}^{\prime} \in \overline{\mathcal{C}}_{\bar{\eta}}, \quad\left|\gamma^{\prime}\right|=|\gamma|+2$ and $n^{\prime}=0$. If $n_{0}^{\prime} \geqslant n_{0}-l_{1}+3$, then $\bar{\eta}^{\prime} \notin \overline{\mathcal{X}}_{2}$ and $\operatorname{EC}\left(\bar{\eta}^{\prime}\right)$ $=\Delta$, while if $n_{0}^{\prime}=n_{0}-l_{1}+2$, then $\bar{\eta}^{\prime} \in \overline{\mathcal{X}} \overline{\mathcal{X}}_{1}$ and $\operatorname{EC}\left(\bar{\eta}^{\prime}\right)=U$.

(iv) Let $\bar{\eta}^{\prime} \in \overline{\mathcal{C}}_{\bar{\eta}},\left|\gamma^{\prime}\right|=|\gamma|+4$ and $n^{\prime}=0$. Then $\bar{\eta}^{\prime} \in \overline{\mathcal{X}} \backslash \overline{\mathcal{X}}_{1}$ and $\operatorname{EC}\left(\bar{\eta}^{\prime}\right)=U$.

(v) Let $\bar{\eta}^{\prime} \in \overline{\mathcal{C}}_{\bar{\eta}}$ and $n^{\prime}=1$. Then $\bar{\eta}^{\prime} \in \overline{\mathcal{X}} \backslash \overline{\mathcal{X}}_{1}$ and $\left|\gamma^{\prime}\right|=|\gamma|$ and $\operatorname{EC}\left(\bar{\eta}^{\prime}\right)=U$.

(vi) If $\left|\gamma^{\prime}\right|>|\gamma|+4$ or $n^{\prime}>1$, then $\bar{\eta}^{\prime} \notin \overline{\mathcal{C}}_{\bar{\eta}}$.

(2) We next consider the following partition of the set $\overline{\mathcal{C}}_{\bar{\eta}}$ : 


$$
\overline{\mathcal{C}}_{\bar{\eta}}=\overline{\mathcal{C}}_{\bar{\eta}, 0} \cup \overline{\mathcal{C}}_{\bar{\eta}, 1 a} \cup \overline{\mathcal{C}}_{\bar{\eta}, 1 b} \cup \overline{\mathcal{C}}_{\bar{\eta}, 2}
$$

where

$$
\begin{aligned}
\overline{\mathcal{C}}_{\bar{\eta}, 0} & =\left\{\bar{\eta}^{\prime} \in \overline{\mathcal{C}}_{\bar{\eta}} \cap \overline{\mathcal{X}}_{2}\right\}, \\
\overline{\mathcal{C}}_{\bar{\eta}, 1 a} & =\left\{\bar{\eta}^{\prime} \in \overline{\mathcal{C}}_{\bar{\eta}} \cap\left(\overline{\mathcal{X}} \backslash \overline{\mathcal{X}}_{1}\right):\left|\gamma^{\prime}\right|=|\gamma|+2, n^{\prime}=0\right\}, \\
\overline{\mathcal{C}}_{\bar{\eta}, 1 b} & =\left\{\bar{\eta}^{\prime} \in \overline{\mathcal{C}}_{\bar{\eta}} \cap\left(\overline{\mathcal{X}}_{\backslash} \backslash \overline{\mathcal{X}}_{1}\right):\left|\gamma^{\prime}\right|=|\gamma|+4, n^{\prime}=0 \text { or }\left|\gamma^{\prime}\right|=|\gamma|, n^{\prime}=1\right\}, \\
\overline{\mathcal{C}}_{\bar{\eta}, 2} & =\left\{\bar{\eta}^{\prime} \in \overline{\mathcal{C}}_{\bar{\eta}} \cap\left(\overline{\mathcal{X}}_{1} \backslash \overline{\mathcal{X}}_{2}\right)\right\} .
\end{aligned}
$$

Definition 7.74: (a) An interval of time $\left[t_{1}, t_{2}\right]$ in $\left[0, \tau_{\overline{\mathcal{C}}}^{\bar{c}} \wedge T_{3} e^{\delta_{1} \beta}\right]$ is an INSTABILITY INTERVAL if $\left.\widetilde{\eta}_{t}\right|_{\Lambda} \notin \overline{\mathcal{X}}_{2}$ for all $t \in\left[t_{1}, t_{2}\right]$.

(b) An interval $\left[t_{1}, t_{2}\right]$ contained in an instability interval is of TYPE 1 if $\tilde{\eta}_{t} \mid \bar{\Lambda} \in \overline{\mathcal{X}} \backslash \overline{\mathcal{X}}_{1}$ for all $t \in\left[t_{1}, t_{2}\right]$ and of TYPE 2 if $\widetilde{\eta}_{t} \mid \bar{\Lambda} \in \overline{\mathcal{X}}_{1} \backslash \overline{\mathcal{X}}_{2}$ for all $t \in\left[t_{1}, t_{2}\right]$. A type 1 interval is of TYPE $1 \mathrm{~A}$ if $\widetilde{\eta}_{t} \mid \bar{\Lambda} \in \overline{\mathcal{C}}_{\bar{\eta}, 1 a}$ and of TYPE 1B if $\widetilde{\eta}_{t} \mid \bar{\Lambda} \in \overline{\mathcal{C}}_{\bar{\eta}, 1 b}$.

(c) An instability interval can be the union of intervals of different types (i.e., $1 a, 1 b$, and 2).

(4) The remaining intervals of time in $\left[0, \tau_{\overline{\mathcal{C}}}^{\bar{\eta}} \wedge T_{3} e^{\delta_{1} \beta}\right]$ that are not instability intervals are called intervals of TYPE 0.

An immediate consequence of the recurrence property of $\left(\widetilde{\eta}_{t}\right)_{t \geqslant 0}$ to the sets $\widetilde{\mathcal{X}}_{1}, \widetilde{\mathcal{X}}_{2}$ is the following:

Lemma 7.75: With probability 1-SES the instability intervals of type 1 are shorter than $T_{1} e^{\delta_{1} \beta}$ and the instability intervals of type 2 are shorter than $T_{2} e^{\delta_{1} \beta}$.

(3) Let us now return to the estimate of $\mathbb{P}\left(\mathbf{A}_{\tilde{\eta}}\right)$. We have

$$
\mathbf{A}_{\tilde{\eta}}=\mathbf{A}_{\tilde{\eta}}^{r} \cup \mathbf{A}_{\tilde{\eta}}^{m},
$$

where

$\mathbf{A}_{\tilde{\eta}}^{r}=\mathbf{A}_{\tilde{\eta}} \cap\{$ the final exit move is due to the arrival of a red particle $\} ;$

$\mathbf{A}_{\tilde{\eta}}^{m}=\mathbf{A}_{\tilde{\eta}} \cap\{$ the final exit move is due to a move inside $\bar{\Lambda}\}$.

By Lemma 7.69, we can estimate $\mathrm{P}\left(\mathbf{A}_{\tilde{\eta}}^{m}\right)$ after decomposing it according to the different kinds of exit through the set

$$
\tilde{\mathcal{C}}_{\bar{\eta}}=\left\{\widetilde{\eta}^{\prime} \in \tilde{X}:\left.\widetilde{\eta}^{\prime}\right|_{\bar{\Lambda}} \in \overline{\mathcal{C}}_{\bar{\eta}}\right\}
$$

by considering the starting point of the final exit move. More precisely, if we abbreviate $\bar{\eta}_{1}$ $=\widetilde{\eta}_{\tau_{\tilde{\mathcal{C}}_{\tilde{\eta}}^{c}-1} \mid \bar{\Lambda}}, \bar{\eta}_{2}=\widetilde{\eta}_{\tau_{\mathcal{\mathcal { C }}_{\tilde{\mathcal{\eta}}}} \mid \bar{\Lambda}}$ and $\Delta \bar{H}_{1,2}=\bar{H}\left(\bar{\eta}_{2}\right)-\bar{H}\left(\widetilde{\eta}_{1}\right)$, then we have

$$
\begin{aligned}
\mathbb{P}\left(\mathbf{A}_{\tilde{\eta}}^{m}\right)= & \mathbb{P}\left(\mathbf{A}_{\tilde{\eta}}^{m} \cap\left\{\bar{\eta}_{1} \in \overline{\mathcal{C}}_{\bar{\eta}, 0}\right\} \cap\left\{\Delta \bar{H}_{1,2}=3 U\right\}\right) \\
& +\mathbb{P}\left(\mathbf{A}_{\tilde{\eta}}^{m} \cap\left\{\bar{\eta}_{1} \in \overline{\mathcal{C}}_{\bar{\eta}, 1 a}\right\} \cap\left\{\Delta \bar{H}_{1,2} \geqslant \Delta\right\}\right) \\
& +\mathbb{P}\left(\mathbf{A}_{\tilde{\eta}}^{m} \cap\left\{\bar{\eta}_{1} \in \overline{\mathcal{C}}_{\bar{\eta}, 1 b}\right\} \cap\left\{\Delta \bar{H}_{1,2} \geqslant U\right\}\right) \\
& +\mathbb{P}\left(\mathbf{A}_{\tilde{\eta}}^{m} \cap\left\{\bar{\eta}_{1} \in \overline{\mathcal{C}}_{\bar{\eta}, 2\}} \cap\left\{\Delta \bar{H}_{1,2} \geqslant \Delta\right\}\right)\right. \\
& +\mathbb{P}\left(\mathbf{A}_{\tilde{\eta}}^{m} \cap\left\{\bar{\eta}_{1} \in \overline{\mathcal{C}}_{\bar{\eta}, 0\}} \cap\left\{\Delta \bar{H}_{1,2}=2 U\right\}\right)\right. \\
& +\mathbb{P}\left(\mathbf{A}_{\tilde{\eta}}^{m} \cap\left\{\bar{\eta}_{1} \in \overline{\mathcal{C}}_{\bar{\eta}, 1 a}\right\} \cap\left\{\Delta \bar{H}_{1,2}=U\right\}\right) .
\end{aligned}
$$


The first term on the rhs of (7.78) can be easily estimated, since on the event $\mathbf{A}_{\tilde{\eta}}^{m} \cap\left\{\Delta \bar{H}_{1,2} \mathbf{A}_{\tilde{\eta}}^{m} \cap\left\{\Delta \bar{H}_{1,2}=3 U\right\}\right.$ we have a move of cost $3 U$ in the interval $\left[0, T_{3} e^{\delta_{1} \beta}\right]$, i.e.,

$$
\mathrm{P}\left(\mathbf{A}_{\tilde{\eta}}^{m} \cap\left\{\bar{\eta}_{1} \in \overline{\mathcal{C}}_{\bar{\eta}, 0} \cap \cap\left\{\Delta \bar{H}_{1,2}=3 U\right\}\right) \leqslant e^{-3 U \beta} T_{3} e^{\delta_{1} \beta} .\right.
$$

The control of the last two terms is postponed to Lemma 7.98. As far as the estimate of the remaining three terms is concerned, we can proceed in a similar way, provided we can estimate the total length of the instability intervals of type $1 \mathrm{a}, 1 \mathrm{~b}, 2$. This means that we need to control the number of instability intervals of the different types via a priori SES probability estimates.

(4) Let us next consider the random times $\tau_{i}<T_{3} e^{\delta_{1} \beta} \wedge \tau_{\tilde{\mathcal{C}}_{\tilde{\eta}}^{c}}, i \in \mathbb{N}$, of arrival in $\bar{\Lambda}$ of red particles. By definition, each $\tau_{i}$ is the initial point of an instability interval of type $1 \mathrm{~b}$. Indeed, by Lemma 7.69, $\tau_{i}<T_{3} e^{\delta_{1} \beta} \wedge \tau_{\tilde{\mathcal{C}}_{\tilde{\eta}}^{c}}$ implies that $\widetilde{\eta}_{\tau_{i}-1} \mid \bar{\Lambda} \in \overline{\mathcal{C}}_{\bar{\eta}, 0}$ and $\widetilde{\eta}_{\tau_{i}} \mid \bar{\Lambda} \in \overline{\mathcal{C}}_{\bar{\eta}, 1 b}$. Namely, the arrival of a red particle during an instability interval produces the exit from the cycle $\widetilde{\mathcal{C}}_{\bar{\eta}}$.

In a similar way, let us denote by $\sigma_{j}, j \in \mathbb{N}$, the random times corresponding to the exit of particles from $\bar{\Lambda}$, (i.e., the appearance of red particles) before the exit from the cycle. These times must be the final point of an instability interval of type $1 \mathrm{~b}$, and $\widetilde{\eta}_{\sigma_{j}+1} \mid \bar{\Lambda} \in \overline{\mathcal{C}}_{\bar{\eta}, 0}$.

The interaction between red particles and particles in $\bar{\Lambda}$ is active only during instability intervals containing subintervals of type $1 \mathrm{~b}$. Now consider a realization of our process in the time interval $\left[0, T_{3} e^{\delta_{1} \beta} \wedge \tau_{\mathcal{C}} \tilde{c}_{\tilde{\eta}}^{c}\right]$. This means, in particular, that we have a realization of instability intervals and of random times $\tau_{i}$ and $\sigma_{j}$. Let us look at the process from the point of view of the red particles: this is a system of independent quasi random walks (QRW) given by the following rules:

-When a red particle enters $\bar{\Lambda}$ at a time $\tau_{i}$, it disappears as red particle during the instability interval starting at $\tau_{i}$. During this interval the red particle can be killed if the final time of this instability interval is not a time $\sigma_{j}$. Otherwise the particle reappears at a time $\sigma_{i}$ at some point in $\partial \bar{\Lambda}$.

-A new particle appears at a time $\sigma_{j}$, which is the final point of an instability interval not starting with a $\tau_{i}$. Call the starting point of such an instability interval a coloration time $\alpha_{k}$.

-Outside $\bar{\Lambda}$ red particles move like IRW's.

Thus, the difference between our process and a process of IRW's not only comes from the fact that particles can be created and annihilated (with a random law), but also from the fact that particles can disappear for random intervals of times when they touch $\bar{\Lambda}$ and can reappear again at a different point in $\partial \bar{\Lambda}$.

(5) More precisely, for each coloration time $\alpha_{k}$ define a quasi random walk ( $\left.\mathrm{QRW}\left(\alpha_{k}\right)_{s}\right)_{s \geqslant 0}$ and a delay time $\mathrm{DT}\left(t, \alpha_{k}\right)$ as follows:

-Look at the particle exiting from $\bar{\Lambda}$ at the end, say $\sigma_{j}$, of the instability interval starting with $\alpha_{k}$. Let $n_{1}$ be its label, $\left(\xi\left(n_{1}\right)_{s}\right)_{s \geqslant 0}$ its path, and $\tau_{\bar{\Lambda} \cup \partial \Lambda_{L_{-}}}\left(n_{1}\right)$ the first time it hits the set $\bar{\Lambda} \cup \partial \Lambda_{l_{-}}$. If $\tau_{\bar{\Lambda} \cup \partial \Lambda_{l_{-}}}\left(n_{1}\right) \geqslant T_{3} e^{\delta_{1} \beta} \wedge \tau_{\mathcal{C}_{\bar{\eta}}^{c}}$, then the QRW $\left(\alpha_{k}\right)$ associated with $\alpha_{k}$ is $\left(\xi\left(n_{1}\right)_{s}\right)_{s \geqslant \sigma_{j}}$, i.e.,

$$
\mathrm{QRW}\left(\alpha_{k}\right)_{s}=\xi\left(n_{1}\right)_{s+\sigma_{j}} \quad \forall s \in\left[0,\left(T_{3} e^{\delta_{1} \beta} \wedge \tau_{\mathcal{C}_{\bar{\eta}}^{c}}\right)-\sigma_{j}\right]
$$

and its delay time is

$$
\mathrm{DT}\left(t, \alpha_{k}\right)=\left(t \wedge \sigma_{j}\right)-\alpha_{k},
$$

corresponding to the time spent in $\bar{\Lambda}$ up to time $t$. 
-If $\tau_{\bar{\Lambda} \cup \partial \Lambda_{l_{-}}}\left(n_{1}\right)<T_{3} e^{\delta_{1} \beta} \wedge \tau_{\mathcal{C}_{\bar{\eta}}^{c}}$ and the particle $n_{1}$ hits $\partial \Lambda_{l_{-}}$before $\bar{\Lambda}$, then the particle is annihilated when exiting $\Lambda_{l_{-}}$. So in this case QRW $\left(\alpha_{k}\right)_{s}=\xi\left(n_{1}\right)_{s+\sigma_{j}} \forall s \in\left[0, \tau_{\partial \Lambda_{l_{-}}}\left(n_{1}\right)-\sigma_{j}\right]$ and $\mathrm{DT}\left(t, \alpha_{k}\right)=\left(t \wedge \sigma_{j}\right)-\alpha_{k}$.

-If $\tau_{\Lambda}^{\bar{\Lambda} \cup \partial \Lambda_{l_{-}}}\left(n_{1}\right)<T_{3} e^{\delta_{1} \beta} \wedge \tau_{\mathcal{C}_{\bar{\eta}}^{c}}$ and the particle $n_{1}$ hits $\bar{\Lambda}$ before $\partial \Lambda_{l_{-}}$, then $\tau_{\bar{\Lambda} \cup \partial \Lambda_{l_{-}}}=\tau_{i_{1}}$ for some index $i_{1}$ and we look at the end of the instability interval starting at $\tau_{i_{1}}$. If this end time is not a time $\sigma_{j^{\prime}}$, then the red particle is annihilated in this instability interval, so QRW $\left(\alpha_{k}\right)_{s}$ $=\xi\left(n_{1}\right)_{s+\sigma_{j}} \forall s \in\left[0, \tau_{i_{1}}-\sigma_{j}\right]$ and $\mathrm{DT}\left(t, \alpha_{k}\right)=\left(t \wedge \sigma_{j}\right)-\alpha_{k}$.

-If the end of the instability interval starting with $\tau_{i_{1}}$ is a time $\sigma_{j_{1}}$, then we look at the red particle exiting at this time from $\bar{\Lambda}$. We let $n_{2}$ be its label (not necessarily equal to $n_{1}$ ), and we follow $\xi_{t}\left(n_{2}\right)$ for $t>\sigma_{j_{1}}$. As before, we have to distinguish between different possibilities. If $\tau_{\bar{\Lambda} \cup \partial \Lambda_{l_{-}}}\left(n_{2}\right) \geqslant T_{3} e^{\delta_{1} \beta} \wedge \tau_{\mathcal{C}_{\bar{\eta}}^{c}}$, then

$$
\operatorname{QRW}\left(\alpha_{k}\right)_{s}= \begin{cases}\xi_{s+\sigma_{j}}\left(n_{1}\right) & \text { for } s \in\left[0, \tau_{i_{1}}-\sigma_{j}\right] \\ \xi_{s+\sigma_{j_{1}}}-\left(\tau_{i_{1}}-\sigma_{j}\right) & \text { for } s \in\left[\tau_{i_{1}}-\sigma_{j}, \quad\left(T_{3} e^{\delta_{1} \beta} \wedge \tau_{\mathcal{C}_{\bar{\eta}}^{c}}\right)-\sigma_{j_{1}}+\tau_{i_{1}}-\sigma_{j}\right],\end{cases}
$$

and

$$
\operatorname{Dt}\left(t, \alpha_{k}\right)=\left\{\begin{array}{l}
\left(t \wedge \sigma_{j}\right)-\alpha_{k} \quad \text { for } t \in\left[\alpha_{k}, \tau_{i_{1}}\right] \\
\sigma_{j}-\alpha_{k}+\left(t \wedge \sigma_{j_{1}}\right)-\tau_{i_{1}} \quad \text { for } t \in\left[\tau_{i_{1}}, T_{3} e^{\delta_{1} \beta} \wedge \tau_{\mathcal{C}^{c}}\right]_{\bar{\eta}}
\end{array}\right.
$$

-Similarly, we can iterate the previous construction in the other cases.

Roughly speaking, the process $\left(\mathrm{QRW}\left(\alpha_{k}\right)_{s}\right)_{s \geqslant 0}$ is obtained by glueing together the pieces of random walk performed by red particles outside $\bar{\Lambda}$, where the paths of two red particles $n_{i}$ and $n_{i+1}$ are glued together if particle $n_{i}$ hits $\bar{\Lambda}$ at the beginning of an instability interval ending with the exit of particle $n_{i+1}$ from $\bar{\Lambda}$. The delay time DT $\left(t, \alpha_{k}\right)$ is defined as the total length of the union of the instability intervals cut out in this glueing procedure up to time $t$. By this construction it is clear that, starting from a configuration in $\widetilde{\mathcal{X}}_{3}$, to each red particle we can associate a creation time $\alpha_{k}$.

(6) By using the above construction of QRW's, we will be able to control:

the number of instability intervals;

the probability of the arrival of a red particle in a given time interval.

To do so, we start with the following observation. For any pair of types $s, s^{\prime}$ with $s \neq s^{\prime}$, we define the transformation cost $\mathrm{TC}\left(s, s^{\prime}\right)$ :

$$
\mathrm{TC}\left(s, s^{\prime}\right)=\min _{\bar{\eta}_{1} \in \overline{\mathcal{C}}_{\bar{\eta}, s^{\prime}}, \bar{\eta}_{2} \in \overline{\mathcal{C}}_{\bar{\eta}, s}}\left[\bar{H}\left(\bar{\eta}_{1}\right)-\bar{H}\left(\bar{\eta}_{2}\right)\right]_{+}
$$

for $s=0,1 \mathrm{a}, 1 \mathrm{~b}, 2$ and $s^{\prime}=1 \mathrm{a}, 1 \mathrm{~b}, 2$.

Lemma 7.85: The following hold:

$$
\begin{gathered}
\operatorname{TC}(0,1 \mathrm{a})=U, \quad \operatorname{TC}(0,1 \mathrm{~b})=\Delta, \quad \operatorname{TC}(0,2)=\infty, \\
\operatorname{TC}(1 \mathrm{a}, 1 \mathrm{~b})=U, \quad \operatorname{TC}(1 \mathrm{a}, 2)=\infty, \\
\operatorname{TC}(1 \mathrm{~b}, 1 \mathrm{a})=0, \quad \operatorname{TC}(1 \mathrm{~b}, 2)=0, \\
\operatorname{TC}(2,1 \mathrm{a})=\infty, \quad \operatorname{TC}(2,1 \mathrm{~b})=U .
\end{gathered}
$$


(The transitions that are not possible in one step get transition cost $\infty$.)

Lemma 7.85 implies that, starting from $\widetilde{\mathcal{C}}_{\bar{\eta}, 0}$, the initial time of an interval of type $1 \mathrm{~b}$ is either a time $\tau_{i}$ or a time corresponding to a move of probability $\leqslant e^{-\Delta \beta}$. An instability interval containing an interval of type $1 \mathrm{~b}$ can also be realized by an interval of type 1a followed by an interval of type 1b. Also in this case Lemma 7.85 implies, by (7.86) and Lemma 7.75, that we have two moves of probability $e^{-U \beta}$ within a time interval of length $e^{\delta_{1} \beta}$. Thus we may conclude that each coloration time $\alpha_{k}$ corresponds to a move (or a couple of moves) of probability smaller than $e^{-\Delta \beta}$.

(7) We have the following a priori estimates.

Lemma 7.87: Let $n\left(T_{3} e^{\delta_{1} \beta}, \Delta\right)$ be the number of moves in $\left[0, T_{3} e^{\delta_{1} \beta}\right]$ of probability $\leqslant e^{-\Delta \beta}$. Then, for all $\delta>0$,

$$
\mathrm{P}\left(n\left(T_{3} e^{\delta_{1} \beta}, \Delta\right)>e^{\left(\delta_{1}+\delta\right) \beta}\right)=\mathrm{SES} .
$$

Lemma 7.87 implies that the number of red particles created in $\left[0, T_{3} e^{\delta_{1} \beta}\right]$ is less than $e^{\left(\delta_{1}+\delta\right) \beta}$ with probability $1-$ SES.

Lemma 7.89: Let $\nu(t)$ be the number of visits to $\bar{\Lambda}$ of a $\mathrm{QRW}$ describing a red particle during a time t. Then

$$
\mathbb{P}\left(\nu(t)>(\log t)^{3}\right)<t e^{-\kappa(\log t)^{2}} .
$$

for some $\kappa>0$.

From Lemmas 7.87 and 7.89 we obtain that with probability $1-$ SES the number of instability intervals containing intervals of type $1 \mathrm{~b}$ is less than $e^{\delta_{2} \beta}$ with $\delta_{2}>\delta_{1}$ arbitrary. In a similar way we can prove the following:

Lemma 7.91: There exist $\delta_{3}\left(\delta_{1}, \delta_{2}, \gamma\right)$, satisfying $\lim _{\delta_{1}, \delta_{2}, \gamma \downarrow 0} \delta_{3}\left(\delta_{1}, \delta_{2}, \gamma\right)=0$, and $\beta_{0}>0$ such that for all $\beta>\beta_{0}$ and $t \leqslant T_{3} e^{\delta_{1} \beta}$ with probability $1-\mathrm{SES}$ :

(i) The total number of instability intervals of type $1 b$ in $\left[0, t \wedge \tau_{\mathcal{C}_{\bar{n}}^{c}}\right]$ is less than $e^{\delta_{3} \beta}$.

(ii) The total number of instability intervals of type la in $\left[0, t \wedge \tau_{\overline{\mathcal{C}}_{\bar{\eta}}^{c}}^{\eta}\right]$ is less than $e^{\left(\Delta-U+\delta_{3}\right) \beta}$.

(iii) The total number of instability intervals $\left[t_{1}, t_{2}\right]$ of type $1 a \stackrel{\eta}{\eta}\left[0, t \wedge \tau_{\mathcal{C}_{-}^{c}}\right]$, such that in $\left[t_{1}\right.$ $\left.-e^{\left(\Delta-U+\delta_{1}+\delta_{3}\right) \beta}, t_{1}\right]$ there exists an interval of type $1 b$, is less than $e^{\delta_{3} \beta}$.

(iv) The total number of instability intervals of type 2 in $\left[0, t \wedge \tau_{\overline{\mathcal{C}}_{\bar{\eta}}^{c}}\right]$ is less than $e^{\delta_{3} \beta}$.

(8) We now return to the estimate of the 2nd, 3rd, and 4th terms on the rhs of (7.78). By applying Lemmas 7.75 and 7.91 we obtain

$$
\begin{aligned}
& \mathbb{P}\left(\mathbf{A}_{\tilde{\eta}}^{m} \cap\left\{\bar{\eta}_{1} \in \overline{\mathcal{C}}_{\bar{\eta}, 1 a}\right\} \cap\left\{\Delta \bar{H}_{1,2} \geqslant \Delta\right\}\right) \leqslant e^{\left(-U+\delta_{1}+\delta_{3}\right) \beta}+\mathrm{SES}, \\
& \mathbb{P}\left(\mathbf{A}_{\tilde{\eta}}^{m} \cap\left\{\bar{\eta}_{1} \in \overline{\mathcal{C}}_{\bar{\eta}, 1 b}\right\} \cap\left\{\Delta \bar{H}_{1,2} \geqslant U\right\}\right) \leqslant e^{\left(-U+\delta_{1}+\delta_{3}\right) \beta}+\mathrm{SES}, \\
& \mathbb{P}\left(\mathbf{A}_{\tilde{\eta}}^{m} \cap\left\{\bar{\eta}_{1} \in \overline{\mathcal{C}}_{\bar{\eta}, 2}\right\} \cap\left\{\Delta \bar{H}_{1,2} \geqslant \Delta\right\}\right) \leqslant e^{\left(U-\Delta+\delta_{1}+\delta_{3}\right) \beta}+\text { SES. }
\end{aligned}
$$

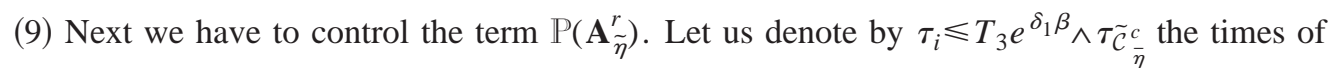
arrival of red particles in $\bar{\Lambda}$ up to time $T_{3} e^{\delta_{1} \beta} \wedge \tau_{\tilde{\mathcal{C}}_{\bar{\eta}}^{c}}$ (including $\tau_{\tilde{\mathcal{C}}_{\bar{\eta}}^{c}}^{c}$ if the exit is due to the arrival before $T_{3} e^{\delta_{1} \beta}$ of a red particle inside an instability interval). We observe that, by Lemmas 7.75 and 7.91, for each $\tau_{i}$ the delay time $\operatorname{DT}\left(T_{3} e^{\delta_{1} \beta} \wedge \tau_{\tilde{\mathcal{C}}^{c}}, \alpha_{k}\right)$ spent by the red particle inside $\bar{\Lambda}$ is less than $e^{\left(U+\delta_{1}+\delta_{3}\right) \beta}$ with probability $1-\mathrm{SES}$, where $\alpha_{k}$ denotes the coloration time of the red particle. 
Lemma 7.93: There exist $\delta_{4}\left(\delta_{1}, \delta_{3}\right)$, satisfying $\lim _{\delta_{1}, \delta_{3} 0} \delta_{4}\left(\delta_{1}, \delta_{3}\right)=0$, and $\beta_{0}>0$ such that, for all $\beta>\beta_{0}, t_{0}>0$ and $T>0$ with $t_{0}+T \leqslant T_{3} e^{\delta_{1} \beta}$,

$$
\mathbb{P}\left(\exists \tau_{i} \in\left[t_{0}, t_{0}+T\right]\right) \leqslant\left[e^{-(\Delta-U) \beta}+e^{-\Delta \beta} T \log t_{0}\right] e^{\delta_{4} \beta} .
$$

Let $\mathbb{P}(1 \mathrm{a}), \mathbb{P}(1 \mathrm{~b}), \mathbb{P}(2)$ be the probabilities of the events where a red particle arrives during an instability interval of types 1a, 1b, 2, respectively. Obviously,

$$
\mathbb{P}\left(\mathbf{A}_{\tilde{\eta}}^{r}\right) \leqslant \mathrm{P}(1 \mathrm{a})+\mathrm{P}(1 \mathrm{~b})+\mathrm{P}(2) .
$$

By using Lemmas 7.75, 7.91, and 7.93, we have

$$
\begin{aligned}
& \mathbb{P}(1 \mathrm{~b}) \leqslant \mathrm{SES}+\mathrm{P}\left(\exists \text { an interval }\left[t_{1}, t_{2}\right] \subset\left[0, T_{3} e^{\delta_{1} \beta} \wedge \tau_{\overline{\mathcal{C}}_{\bar{\eta}}^{c}}\right] \text { of type } 1 \mathrm{~b}\right. \\
& \text { with } \left.t_{2}-t_{1} \leqslant e^{\delta_{1}, \beta} \text { and } \exists \tau_{i} \in\left(t_{1}, t_{2}\right)\right) \\
& \leqslant \mathrm{SES}+2 e^{-\left(\Delta-U-\delta_{3}-\delta_{4}\right) \beta}, \\
& \mathbb{P}(2) \leqslant \operatorname{SES}+\mathbb{P}\left(\exists \text { an interval }\left[t_{1}, t_{2}\right] \subset\left[0, T_{3} e^{\delta_{1} \beta} \wedge \tau_{\tau_{\frac{\eta}{\eta}}^{c}}\right] \text { of type } 2\right. \\
& \text { with } \left.t_{2}-t_{1} \leqslant U \beta+\delta_{1} \beta \text { and } \exists \tau_{i} \in\left(t_{1}, t_{2}\right)\right) \\
& \leqslant \mathrm{SES}+2 e^{-\left(\Delta-u-\delta_{1}-\delta_{3}-\delta_{4}\right) \beta} .
\end{aligned}
$$

As far as the term $\mathbb{P}(1 \mathrm{a})$ is concerned, we have to distinguish between two cases depending on the existence of an interval of type $1 \mathrm{~b}$ at a distance less than $e^{\left(U+\delta_{1}+\delta_{3}\right) \beta}$ from the interval of type 1a we are considering:

$$
\begin{aligned}
& \mathbb{P}\left(1 \mathrm{a} \cap\left\{\exists \text { interval of type } 1 \mathrm{~b} \text { in }\left[t_{1}-e^{\left(U+\delta_{1}+\delta_{3}\right) \beta}, t_{1}\right]\right\}\right) \leqslant 2 e^{-\left(\Delta-U-\delta_{3}-\delta_{4}\right) \beta}, \\
& \mathbb{P}\left(1 \mathrm{a} \cap\left\{\nexists \text { interval of type } 1 \mathrm{~b} \text { in }\left[t_{1}-e^{\left(U+\delta_{1}+\delta_{3}\right) \beta}, t_{1}\right]\right\}\right) \leqslant e^{-\left(\Delta-U-\delta_{1}-\delta_{3}-\delta_{4}\right) \beta} .
\end{aligned}
$$

Indeed, in this last case the first term in the estimate in Lemma 7.93 is absent, since it comes from the event that there exists a creation time $\alpha_{k}$ at a distance less than $e^{\left(U+\delta_{1}+\delta_{3}\right) \beta}$ from the interval of type 1a we are considering and this is forbidden by the event $\left\{\nexists\right.$ interval of type $1 \mathrm{~b}$ in $\left[t_{1}\right.$ $\left.\left.-e^{\left(U+\delta_{1}+\delta_{3}\right) \beta}, t_{1}\right]\right\}$. [See (7.119) in the proof of Lemma 7.93 below.]

(10) It remains to estimate the probability of exit by contraction. This is given by the following.

Lemma 7.98: There exists $\delta_{5}(\gamma)$ satisfying $\lim _{\gamma \downarrow 0} \delta_{5}(\gamma)=0$ and $\beta_{0}>0$ such that, for all $\beta$ $>\beta_{0}$

$$
\begin{aligned}
& P\left(\mathbf{A}_{\tilde{\eta}}^{m} \cap\left\{\widetilde{\eta}_{1} \in \overline{\mathcal{C}}_{\bar{\eta}, 0\}} \cap\left\{\Delta \bar{H}_{1,2}=2 U\right\}\right)+P\left(\mathbf{A}_{\bar{\eta}}^{m} \cap\left\{\bar{\eta}_{1} \in \overline{\mathcal{C}}_{\bar{\eta}, 1 a}\right\} \cap\left\{\Delta \bar{H}_{1,2}=U\right\}\right)\right. \\
& \quad \leqslant e^{-\left[(2 U-\Delta)\left(l_{1}-1\right)-\delta_{5}\right] \beta} .
\end{aligned}
$$

This completes the proof of Proposition 7.43 for the case $\bar{\eta} \in \overline{\mathcal{X}}_{3} \backslash \mathcal{L}$. QED

(11) For the case $\bar{\eta} \in \mathcal{L}$ we can proceed in a similar way. We indicate here only the differences with the case $\bar{\eta} \in \overline{\mathcal{X}}_{3} \backslash \mathcal{L}$.

The characterization of the set $\overline{\mathcal{C}}_{\bar{\eta}}^{2 U}$ can be done as follows. By Lemma 5.13 (iii), we know that $\mathcal{F}\left(\overline{\mathcal{C}}_{\bar{\eta}}^{2 U}\right)=\overline{\mathcal{C}}_{\bar{\eta}}^{2 U} \cap \overline{\mathcal{X}}_{3}=\overline{\mathcal{C}}_{\bar{\eta}}^{2 U} \cap \overline{\mathcal{X}}_{2}$. A direct check shows that $\bar{H}\left(\bar{\eta}^{\prime}\right) \in\{\bar{H}(\bar{\eta}), \bar{H}(\bar{\eta})+\Delta$ $-U, \bar{H}(\bar{\eta})+U, \bar{H}(\bar{\eta})+\Delta\}$ for any $\bar{\eta}^{\prime} \in \overline{\mathcal{C}}_{\bar{\eta}}^{2 U}$. Moreover, configurations with $\bar{H}\left(\bar{\eta}^{\prime}\right) \geqslant \bar{H}(\bar{\eta})+U$ 
are not in $\overline{\mathcal{X}}_{1}$. Indeed, if such an $\bar{\eta}^{\prime}$ is not 0-reducible, then this inequality implies that for each $\bar{\eta}^{\prime \prime}$ with $\bar{H}\left(\bar{\eta}^{\prime \prime}\right)<\bar{H}\left(\bar{\eta}^{\prime}\right)$ we have $\bar{H}\left(\bar{\eta}^{\prime}, \bar{\eta}^{\prime \prime}\right) \geqslant \bar{H}\left(\bar{\eta}^{\prime}\right)+U$, and so if we choose $\bar{\eta}^{\prime \prime}=\bar{\eta}$, then we obtain $\bar{H}\left(\bar{\eta}^{\prime}, \bar{\eta}\right) \geqslant \bar{H}(\bar{\eta})+U+U$, which contradicts $\bar{\eta}^{\prime} \in \overline{\mathcal{C}}_{\bar{\eta}}^{2 U}$.

Thus the partition of the set $\overline{\mathcal{C}}_{\bar{\eta}}^{2 U}$ uses

$$
\begin{aligned}
& \overline{\mathcal{C}}_{\bar{\eta}, 0}^{2 U}=\left\{\bar{\eta}^{\prime} \in \overline{\mathcal{C}}_{\bar{\eta}}^{2 U} \cap \overline{\mathcal{X}}_{3}\right\}, \\
& \overline{\mathcal{C}}_{\bar{\eta}, 1 a}^{2 U}=\left\{\bar{\eta}^{\prime} \in \overline{\mathcal{C}}_{\bar{\eta}}^{2 U} \cap\left(\overline{\mathcal{X}} \overline{\mathcal{X}}_{1}\right) \text { with } \bar{H}\left(\bar{\eta}^{\prime}\right)=\bar{H}(\bar{\eta})+U\right\}, \\
& \overline{\mathcal{C}}_{\bar{\eta}, 1 b}^{2 U}=\left\{\bar{\eta}^{\prime} \in \overline{\mathcal{C}}_{\bar{\eta}}^{2 U} \cap\left(\overline{\mathcal{X}}_{\mathcal{X}_{1}}\right) \text { with } \bar{H}\left(\bar{\eta}^{\prime}\right)=\bar{H}(\bar{\eta})+\Delta\right\}, \\
& \overline{\mathcal{C}}_{\bar{\eta}, 2}^{2 U}=\left\{\bar{\eta}^{\prime} \in \overline{\mathcal{C}}_{\bar{\eta}}^{2 U} \cap\left(\overline{\mathcal{X}}_{1} \backslash \overline{\mathcal{X}}_{2}\right) \text { with } \bar{H}\left(\bar{\eta}^{\prime}\right)=\bar{H}(\bar{\eta})+\Delta-U\right\} .
\end{aligned}
$$

The exit costs in this case are given by

$$
\begin{array}{ll}
\bar{\eta}^{\prime} \in \overline{\mathcal{C}}_{\bar{\eta}, 0}^{2 U}: & \operatorname{EC}\left(\bar{\eta}^{\prime}\right)=2 U, \\
\bar{\eta}^{\prime} \in \overline{\mathcal{C}}_{\bar{\eta}, 1 a}^{2 U}: & \operatorname{EC}\left(\bar{\eta}^{\prime}\right)=U, \\
\bar{\eta}^{\prime} \in \overline{\mathcal{C}}_{\bar{\eta}, 1 b}^{2 U}: & \operatorname{EC}\left(\bar{\eta}^{\prime}\right)=U, \\
\bar{\eta}^{\prime} \in \overline{\mathcal{C}}_{\bar{\eta}, 2}^{2 U}: & \operatorname{EC}\left(\bar{\eta}^{\prime}\right)=\Delta,
\end{array}
$$

where we use that $2 U-\Delta<U / 2$. The transition costs are given by

$$
\begin{aligned}
& \mathrm{TC}(0,1 \mathrm{a})=U, \quad \operatorname{TC}(0,1 \mathrm{~b})=\Delta, \quad \operatorname{TC}(0,2)=\infty, \\
& \operatorname{TC}(1 \mathrm{a}, 1 \mathrm{~b})=\infty, \quad \operatorname{TC}(1 \mathrm{a}, 2)=\infty, \quad \operatorname{TC}(1 \mathrm{~b}, 1 \mathrm{a})=\infty, \\
& \mathrm{TC}(1 \mathrm{~b}, 2)=0, \quad \mathrm{TC}(2,1 \mathrm{a})=\infty, \quad \mathrm{TC}(2,1 \mathrm{~b})=U .
\end{aligned}
$$

We can prove also for this case the result in Lemma 7.91, and the rest of the proof follows exactly the same calculations performed for the case $\bar{\eta} \in \overline{\mathcal{X}}_{3} \backslash \mathcal{L}$.

\subsection{Proof of the lemmas in Sec. 7.7}

In this section we prove the lemmas that were used in Sec. 7.7.

Proof of Lemma 7.69: (i) Let $l_{1}, l_{2}$ be the side lengths of $\eta$, with $0 \leqslant l_{2}-l_{1} \leqslant 1$, and let $\gamma$ be its contour. For any $\bar{\eta}^{\prime}$ such that $\left|\gamma^{\prime}\right|<|\gamma|$, we have $n_{0}^{\prime} \leqslant n_{0}-l_{1}$. On the other hand, by the results in Sec. 4 we know that $\bar{H}\left(\mathcal{N}_{l_{1} l_{2}}, \mathcal{N}_{l_{1}\left(l_{2}-1\right)}\right) \geqslant r(\bar{\eta})+\Delta+\bar{H}(\bar{\eta})$, so that such a configuration $\bar{\eta}^{\prime}$ is not in $\overline{\mathcal{C}}_{\bar{\eta}}$. In the same way, by using the results in Sec. 4, we immediately see that if $n_{0}^{\prime} \geqslant n_{0}$ +2 or $n_{0}^{\prime} \leqslant n_{0}-l_{1}+1$, then the configuration $\bar{\eta}^{\prime}$ is not in $\overline{\mathcal{C}}_{\bar{\eta}}$.

(ii) Equation (7.70) follows from (i) and the fact that $n_{0}$ is the maximal number of internal particles given a contour of length $|\gamma|$. To prove that $\eta^{\prime} \in \overline{\mathcal{X}}_{2}$, we only have to observe that $\gamma^{\prime}$ must be a monotone contour, after which we can apply Proposition 5.11. To evaluate the exist cost of $\eta^{\prime}$, note that

$$
\bar{H}\left(\eta^{\prime}\right)=\bar{H}(\bar{\eta})+l^{\prime}(2 U-\Delta),
$$


while, by the definition of EC in (7.68),

$$
\bar{H}\left(\bar{\eta}^{\prime}\right)+\mathrm{EC}\left(\bar{\eta}^{\prime}\right)-\bar{H}(\bar{\eta}) \geqslant r(\bar{\eta})+\Delta \geqslant\left(l_{1}-1\right)(2 U-\Delta)+\Delta .
$$

So we obtain

$$
\mathrm{EC}\left(\bar{\eta}^{\prime}\right) \geqslant\left(l_{1}-1-l^{\prime}\right)(2 U-\Delta)+\Delta .
$$

(iii) We argue by contradiction. Suppose that $\left|\gamma^{\prime}\right|=|\gamma|+2, n^{\prime}=0$ and $\bar{\eta}^{\prime} \in \overline{\mathcal{C}}_{\bar{\eta}} \cap \overline{\mathcal{X}}_{2}$. Then, since $n_{0}^{\prime} \leqslant n_{0}+1$, we have

$$
\bar{H}\left(\bar{\eta}^{\prime}\right) \geqslant \bar{H}(\bar{\eta})+U-(2 U-\Delta) .
$$

But

$$
\bar{H}\left(\bar{\eta}^{\prime}, \bar{\eta}\right)-\bar{H}\left(\bar{\eta}^{\prime}\right) \geqslant \Delta .
$$

Hence

$$
\bar{H}\left(\bar{\eta}^{\prime}, \bar{\eta}\right) \geqslant \Delta+\Delta-U+\bar{H}(\bar{\eta}) \geqslant r(\bar{\eta})+\bar{H}(\bar{\eta}),
$$

which contradicts the hypothesis $\bar{\eta}^{\prime} \in \overline{\mathcal{C}}_{\bar{\eta}}$. So $\bar{\eta}^{\prime} \notin \overline{\mathcal{X}}_{2}$. To compute $\operatorname{EC}\left(\bar{\eta}^{\prime}\right)$, we note that

$$
\mathrm{EC}\left(\bar{\eta}^{\prime}\right) \geqslant \bar{H}(\bar{\eta})-\bar{H}\left(\bar{\eta}^{\prime}\right)+r(\bar{\eta})+\Delta,
$$

and since

$$
\bar{H}(\bar{\eta})-\bar{H}\left(\bar{\eta}^{\prime}\right)=(-2 U+\Delta)\left(n_{0}-n_{0}^{\prime}\right)-U,
$$

we obtain the result.

(iv) In a similar way, $\bar{H}\left(\bar{\eta}^{\prime}\right) \geqslant \bar{H}(\bar{\eta})+2 U-(2 U-\Delta)$, and if $\bar{\eta}^{\prime} \in \overline{\mathcal{X}}_{1}$, then

$$
\bar{H}\left(\bar{\eta}^{\prime}, \bar{\eta}\right) \geqslant \Delta+U+\bar{H}(\bar{\eta}) \geqslant r(\bar{\eta})+\bar{H}(\bar{\eta}) .
$$

Now $\operatorname{EC}\left(\bar{\eta}^{\prime}\right)=U$, since

$$
\bar{H}\left(\bar{\eta}^{\prime}\right)+U-\bar{H}(\bar{\eta}) \geqslant \Delta+U \geqslant r(\bar{\eta})+\Delta .
$$

(v) Note that

$$
\bar{H}\left(\bar{\eta}^{\prime}\right)=(-2 U+\Delta) n_{0}^{\prime}+\frac{U}{2}\left|\gamma^{\prime}\right|+\Delta \geqslant(-2 U+\Delta)+\frac{U}{2}\left(\left|\gamma^{\prime}\right|-|\gamma|\right)+\Delta+\bar{H}(\bar{\eta}),
$$

so if $\left|\gamma^{\prime}\right|>|\gamma|$, then

$$
\bar{H}\left(\bar{\eta}^{\prime}\right) \geqslant-2 U+\Delta+U+\Delta+\bar{H}(\bar{\eta})=2 \Delta-U+\bar{H}(\bar{\eta}) \geqslant r(\bar{\eta})+\bar{H}(\bar{\eta}),
$$

while if $\left|\gamma^{\prime}\right|=|\gamma|$ and $\bar{\eta}^{\prime} \in \overline{\mathcal{X}}_{1}$, then

$$
\bar{H}\left(\bar{\eta}^{\prime}, \bar{\eta}\right) \geqslant U+\bar{H}\left(\bar{\eta}^{\prime}\right) \geqslant U+2 \Delta-2 U+\bar{H}(\bar{\eta}) \geqslant r(\bar{\eta})+\bar{H}(\bar{\eta}) .
$$

Also in this case we have that $\mathrm{EC}\left(\bar{\eta}^{\prime}\right)=U$, since $\bar{H}\left(\bar{\eta}^{\prime}\right)+U-\bar{H}(\bar{\eta}) \geqslant r(\bar{\eta})+\Delta$. 
(vi) If $\left|\gamma^{\prime}\right|>|\gamma|+4$ or $n^{\prime}>1$, then $\bar{H}\left(\bar{\eta}^{\prime}\right) \geqslant U+\Delta+\bar{H}(\bar{\eta}) \geqslant r(\bar{\eta})+\bar{H}(\bar{\eta})$ or $\bar{H}\left(\bar{\eta}^{\prime}\right) \geqslant 3 \Delta$ $-2 U+\bar{H}(\bar{\eta}) \geqslant r(\bar{\eta})+\bar{H}(\bar{\eta})$. QED

Proof of Lemma 7.75: The claim is an immediate consequence of Proposition 6.2. QED

Proof of Lemma 7.85:

(1) Let $s=0, s^{\prime}=1 \mathrm{a}$. Then $\left|\gamma_{2}\right|=\left|\gamma_{1}\right|+2$ and $n_{0,1}=n_{0,2}$, since the move $\bar{\eta}_{1} \rightarrow \bar{\eta}_{2}$ has to be inside $\bar{\Lambda}_{0}$. Therefore $\bar{H}\left(\bar{\eta}_{2}\right)-\bar{H}\left(\bar{\eta}_{1}\right)=U$. Analogously, let $s=0, s^{\prime}=1 \mathrm{~b}$. Then $\left|\gamma_{2}\right|=\left|\gamma_{1}\right|+4$ and $n_{0,2}=n_{0,1}$ or $\left|\gamma_{2}\right|=\left|\gamma_{1}\right|$ and $n_{2}=1$, so that $\bar{H}\left(\bar{\eta}_{2}\right)-\bar{H}\left(\bar{\eta}_{1}\right)=2 U \wedge \Delta=\Delta$.

(2) The transition $\bar{\eta}_{1} \in \overline{\mathcal{C}}_{\bar{\eta}, 0} \rightarrow \bar{\eta}_{2} \in \overline{\mathcal{C}}_{\bar{\eta}, 2}$ is not possible. Indeed, we have $\bar{H}\left(\bar{\eta}_{1}\right) \neq \bar{H}\left(\bar{\eta}_{2}\right)$, since $\bar{\eta}_{1}$ is not 0-equivalent to $\bar{\eta}_{2}$, and if $\bar{H}\left(\bar{\eta}_{1}\right)<\bar{H}\left(\bar{\eta}_{2}\right)$, then the move $\bar{\eta}_{2} \rightarrow \bar{\eta}_{1}$ shows that $\bar{\eta}_{2} \notin \overline{\mathcal{X}}_{1}$, while if $\bar{H}\left(\bar{\eta}_{1}\right)>\bar{H}\left(\bar{\eta}_{2}\right)$, then the move $\bar{\eta}_{1} \rightarrow \bar{\eta}_{2}$ shows that $\bar{\eta}_{1} \notin \overline{\mathcal{X}}_{1}$.

(3) If $s=1 \mathrm{a}, s^{\prime}=1 \mathrm{~b}$, then we have two possibilities:

(1) $\left|\gamma_{2}\right|=\left|\gamma_{1}\right|+2$ and $n_{1}=n_{2}=0, n_{0,1}=n_{0,2}$;

(2) $\left|\gamma_{2}\right|=|\gamma|,\left|\gamma_{1}\right|=|\gamma|+2$ and $n_{2}=1, n_{1}=0$.

In the first case $\bar{H}\left(\bar{\eta}_{2}\right)-\bar{H}\left(\bar{\eta}_{1}\right)=U$, in the second case the move must be between $\bar{\Lambda}_{0}$ and the ring $\bar{\Lambda} \backslash \bar{\Lambda}_{0}$, so $n_{0,2}=n_{0,1}-1$ and $\bar{H}\left(\bar{\eta}_{2}\right)-\bar{H}\left(\bar{\eta}_{1}\right)=(-2 U+\Delta)\left(n_{0,2}-n_{0,1}\right)+(U / 2)\left(\left|\gamma_{2}\right|-\left|\gamma_{1}\right|\right)$ $+\Delta=U$.

(4) The transition $\bar{\eta}_{1} \in \overline{\mathcal{C}}_{\bar{\eta}, 1 a} \rightarrow \bar{\eta}_{2} \in \overline{\mathcal{C}}_{\bar{\eta}, 2}$ is not possible. Indeed, we have $\bar{H}\left(\bar{\eta}_{1}\right) \neq \bar{H}\left(\bar{\eta}_{2}\right)$, since $\bar{\eta}_{1}$ is not 0 -equivalent to $\bar{\eta}_{2}$, and $\left|\gamma_{1}\right|=|\gamma|+2=\left|\gamma_{2}\right|$ and $n_{0,1}=n_{0,2}$, since $n_{1}=n_{2}=0$, but this is a contradiction.

(5) If $s=1 \mathrm{~b}, s^{\prime}=2$, then we have a creation cost zero (as given, for instance, by the transition in which a particle reaches the square or quasi-square cluster). A similar argument holds for the case $s=1 \mathrm{~b}, s^{\prime}=1 \mathrm{a}$.

(6) The transition $2 \rightarrow 1 \mathrm{a}$ is forbidden by an argument similar to that used in the transition $1 \mathrm{a} \rightarrow 2$.

(7) If $s=2, s^{\prime}=1 \mathrm{~b}$, then we have two possibilities:

(1) $\left|\gamma_{2}\right|=|\gamma|+4,\left|\gamma_{1}\right|=|\gamma|+2$ and $n_{1}=n_{2}=0$;

(2) $\left|\gamma_{2}\right|=|\gamma|,\left|\gamma_{1}\right|=|\gamma|+2$ and $n_{2}=1, n_{1}=0$.

In both cases we have $\bar{H}\left(\bar{\eta}_{2}\right)-\bar{H}\left(\bar{\eta}_{1}\right)=U$. QED

Proof of Lemma 7.87: This is an elementary large deviation estimate for a binomial distribution obtained by applying an exponential Chebyshev inequality. QED

Proof of Lemma 7.89: For any $t>1$ :

$$
\begin{aligned}
& \mathbb{P}\left(\nu(t)>(\log t)^{3}\right) \\
& \quad \leqslant \mathbb{P}\left(\exists \alpha_{k} \in[0, t]: \exists(\log t)^{3} \text { IRW's starting at } \partial \bar{\Lambda} \text { and returning to } \bar{\Lambda} \text { within time } t\right) \\
& \quad \leqslant t\left(1-\frac{\kappa}{\log t}\right)^{(\log t)^{3}} \leqslant t e^{-\kappa(\log t)^{2}},
\end{aligned}
$$

where we use Proposition 3.2. QED

Proof of Lemma 7.91:

(i) Let $N^{\prime}(1 \mathrm{~b})$ be the number of instability intervals containing at least an interval of type $1 \mathrm{~b}$ within time $T_{3} e^{\delta_{1} \beta} \wedge \tau_{\mathcal{C}^{c}} \cdot$. For any $\delta_{2}>\delta_{1}$, let $\delta=\left(\delta_{2}-\delta_{1}\right) / 2$. We have 


$$
\begin{aligned}
\mathbb{P}\left(N^{\prime}(1 \mathrm{~b})>e^{\delta_{2} \beta}\right) \leqslant & \mathbb{P}\left(n\left(T_{3} e^{\delta_{1} \beta}, \Delta\right)>e^{\left(\delta_{1}+\delta\right) \beta}\right)+\mathbb{P}\left(\nu\left(T_{3} e^{\delta_{1} \beta}\right)>e^{\delta \beta}\right) \\
& +\mathbb{P}\left(\left\{N^{\prime}(1 \mathrm{~b})>e^{\delta_{2} \beta}\right\} \cap\left\{n\left(T_{3} e^{\delta_{1} \beta}, \Delta\right) \leqslant e^{\left(\delta_{1}+\delta\right) \beta}\right\} \cap\left\{\nu\left(T_{3} e^{\delta_{1} \beta}\right)>e^{\delta \beta}\right\}\right) .
\end{aligned}
$$

By using Lemmas 7.87 and 7.89 , we get $\mathbb{P}\left(N^{\prime}(1 \mathrm{~b})>e^{\delta_{2} \beta}\right) \leqslant$ SES.

In any instability interval containing intervals of type $1 \mathrm{~b}$, the maximal number of intervals of type $1 \mathrm{~b}$ is $e^{\left(\delta_{1}+\delta\right) \beta}$ with probability $1-\mathrm{SES}$ for any $\delta>0$. This follows from Lemma 7.85 and the analogue of Lemma 7.87 for the quantity $n\left(T_{2} e^{\delta_{1} \beta}, U\right)$. Thus, we have that the total number $N(1 \mathrm{~b})$ of intervals of type $1 \mathrm{~b}$ within time $T_{3} e^{\delta_{1} \beta} \wedge \tau_{\overline{\mathcal{C}}^{\bar{\eta}}}^{\bar{c}}$ is smaller than $e^{\delta_{3} \beta}$ with probability 1 - SES for $\delta_{3}>2 \delta_{1}$.

(ii) With similar arguments we can conclude that the number $N^{\prime}$ (1a) of intervals of type 1a starting from an interval of type 0 is, with probability $1-\mathrm{SES}$, smaller than $e^{\left(\Delta-U+\delta_{1}+\delta\right) \beta}$, and the number of intervals of type 1a contained in an instability interval containing intervals of type $1 \mathrm{~b}$ is less than $e^{\delta \beta}$, for any $\delta>0$, so that $N(1 \mathrm{a}) \leqslant N^{\prime}(1 \mathrm{a})+N^{\prime}(1 \mathrm{~b}) e^{\delta \beta}$.

(iii) By using that $N(1 \mathrm{~b}) \leqslant e^{\delta_{3} \beta}$, we can estimate the number of intervals of type 1a contained in a union of intervals with total length $e^{\left(\Delta-U+\delta_{1}+2 \delta_{3}\right) \beta}$ by using the same argument as in (ii). 7.85 .

(iv) The number $N(2)$ of intervals of type 2 is estimated by $N(2) \leqslant N(1 \mathrm{~b})$, again by Lemma

QED

Proof of Lemma 7.93: (1) Let $\alpha_{k}$ be a coloration time. If $t_{0} \leqslant e^{U \beta+\left(\delta_{1}+\delta_{3}\right) \beta}$, then

$$
\begin{aligned}
& \mathbb{P}\left(\exists \tau_{i} \in\left[t_{0}, t_{0}+T\right]\right) \leqslant \mathbb{P}\left(\exists \alpha_{k} \in\left[0, e^{U \beta+\left(\delta_{1}+\delta_{3}\right) \beta}+T\right]\right) \\
& e^{\delta_{3} \beta} e^{U \beta+\left(\delta_{1}+\delta_{3}\right) \beta}+T \\
& \leqslant \sum_{k=1} \quad \sum_{s=0} \quad \mathrm{P}\left(\alpha_{k}=s\right)+\mathrm{SES} \\
& \leqslant e^{-\Delta \beta+\delta_{3} \beta}\left(e^{U \beta+\left(\delta_{1}+\delta_{3}\right) \beta}+T\right)+\mathrm{SES} .
\end{aligned}
$$

On the other hand, if $t_{0}>e^{U \beta+\left(\delta_{1}+\delta_{3}\right) \beta}$, then

$$
\begin{aligned}
& \mathbb{P}\left(\exists \tau_{i} \in\left[t_{0}, t_{0}+T\right]\right) \\
&= \mathbb{P}\left(\left\{\exists \tau_{i} \in\left[t_{0}, t_{0}+T\right]\right\} \cap\left\{\alpha_{k} \in\left[t_{0}-e^{U \beta+\left(\delta_{1}+\delta_{3}\right) \beta}, t_{0}+T\right]\right\}\right) \\
&+ \mathbb{P}\left(\left\{\exists \tau_{i} \in\left[t_{0}, t_{0}+T\right]\right\} \cap\left\{\alpha_{k}<t_{0}-e^{U \beta+\left(\delta_{1}+\delta_{3}\right) \beta}\right\}\right) \\
& \leqslant \sum_{k=1}^{\delta_{3} \beta} \sum_{s=t_{0}-e^{U \beta+\left(\delta_{1}+\delta_{3}\right) \beta}}^{t_{0}+T} \mathbb{P}\left(\alpha_{k}=s\right)+\sum_{k=1}^{e^{\delta_{3} \beta}} \sum_{s=0} \sum_{t=t_{0}-e^{U \beta+\left(\delta_{1}+\delta_{3}\right) \beta}}^{t_{0}+T} \\
& \times \mathbb{P}\left(\left\{\alpha_{k}=s\right\} \cap\left\{\mathrm{QRW}{ }_{t-s-\mathrm{DT}\left(t, \alpha_{k}\right)}\left(\alpha_{k}\right) \in \bar{\Lambda}\right\}\right)+\mathrm{SES} .
\end{aligned}
$$

(2) For QRW similar estimates hold as for SRW, namely,

$$
\max _{x} \mathrm{P}\left(\mathrm{QRW}_{t}=x\right) \leqslant \frac{\kappa \log t}{t} .
$$

Indeed, we can write

$$
\mathrm{QRW}_{t}=\mathrm{SRW}_{t}+\mathrm{JP}_{t},
$$

where $\mathrm{JP}_{t}$ is a sum of jumps 


$$
\mathrm{JP}_{t}=\sum_{n=0}^{\nu(t)} J_{n}
$$

with $\left|J_{n}\right|<2 l_{0}$ and with $\nu(t)$ estimated in Lemma 7.89.

(3) From (7.120), using that $\mathrm{DT}\left(T_{3} e^{\delta_{1} \beta} \wedge \tau_{\mathcal{C}_{\bar{\eta}}^{c}}, \alpha_{k}\right)<e^{\left(U+\delta_{1}+\delta_{3}\right) \beta}$ with probability $1-\mathrm{SES}$, we obtain

$$
\begin{aligned}
& \mathbb{P}\left(\exists \tau_{i} \in\left[t_{0}, t_{0}+T\right]\right) \\
& \leqslant e^{-\left(\Delta-\delta_{3}\right) \beta}\left(e^{U \beta+\left(\delta_{1}+\delta_{3}\right) \beta}+T\right) \\
& \quad+\sum_{t=t_{0}}^{t_{0}+T} \sum_{s=0} t_{0}-e^{U \beta+\left(\delta_{1}+\delta_{3}\right) \beta} \\
& \leqslant
\end{aligned}
$$

Proof of Lemma 7.98:

(1) For $m=n_{0}-l_{1}+2, \ldots, n_{0}+1$, define

$$
\overline{\mathcal{C}}_{\bar{\eta}}(m)=\overline{\mathcal{C}}_{\bar{\eta}} \cap \mathcal{N}_{m},
$$

where $\mathcal{N}_{m}=\left\{\bar{\eta}^{\prime} \in \overline{\mathcal{X}}: n_{0}^{\prime}+n^{\prime}=m\right\}$. By Lemma 7.69, $\overline{\mathcal{C}}_{\bar{\eta}}=\cup_{m=n_{0}-l_{1}+2}^{n_{0}+1} \overline{\mathcal{C}}_{\bar{\eta}}(m)$. We note that the sets $\bar{C}_{\bar{\eta}}(m)$ are not necessarily connected. We write

$$
\overline{\mathcal{C}}_{\bar{\eta}}(>m)=\bigcup_{m^{\prime}=m+1}^{n_{0}+1} \overline{\mathcal{C}} \bar{\eta}\left(m^{\prime}\right) .
$$

Let $\zeta_{j}(m), j \in \mathbb{N}$, denote the return times to the set $\overline{\mathcal{C}}_{\bar{\eta}}(m)$ within $T_{3} e^{\delta_{1} \beta} \wedge \tau_{\overline{\mathcal{C}}_{\bar{\eta}}^{c}}$, i.e.,

$$
\begin{aligned}
& \zeta_{1}(m)=\left(T_{3} e^{\delta_{1} \beta} \wedge \tau_{\overline{\mathcal{C}}_{\bar{\eta}}}^{c}\right) \wedge \min \left\{t \geqslant 0: \widetilde{\eta}_{t} \in \overline{\mathcal{C}}_{\bar{\eta}}(m)\right\}, \\
& \zeta_{j}^{\prime}(m)=\left(T_{3} e^{\delta_{1} \beta} \wedge \tau_{\mathcal{C}_{\bar{\eta}}}^{\bar{c}}\right) \wedge \min \left\{t>\zeta_{j}(m): \widetilde{\eta}_{t} \neq \overline{\mathcal{C}}_{\bar{\eta}}(m)\right\}, \\
& \zeta_{j+1}(m)=\left(T_{3} e^{\delta_{1} \beta} \wedge \tau_{\overline{\mathcal{C}}_{\bar{\eta}}}{ }_{\bar{\eta}}\right) \wedge \min \left\{t>\zeta_{j}^{\prime}(m): \widetilde{\eta}_{t} \in \overline{\mathcal{C}}_{\bar{\eta}}(m)\right\} .
\end{aligned}
$$

(2) Let $m_{0}$ be the minimal index $m$, i.e., $m_{0}=n_{0}-l_{1}+2$. We say that the process $\left(\tilde{\eta}_{t}\right)_{t \geqslant 0}$ exits by contraction from the set $\overline{\mathcal{C}}_{\bar{\eta}}\left(m_{0}\right)$ if it exits without increasing the number of particles in $\bar{\Lambda}$, i.e., if $\Sigma_{x \in \bar{\Lambda}} \widetilde{\eta}_{t}(x)=m_{0}$ for all $t \leqslant \tau_{\mathcal{C}_{\bar{\eta}}^{c}}^{c}\left(m_{0}\right)$. On the events

$$
\begin{aligned}
& \mathbf{A}_{\tilde{\eta}}^{m} \cap\left\{\bar{\eta}_{1} \in \overline{\mathcal{C}}_{\bar{\eta}, 0}\right\} \cap\left\{\Delta \bar{H}_{1,2}=2 U\right\}, \\
& \mathbf{A}_{\tilde{\eta}}^{m} \cap\left\{\bar{\eta}_{1} \in \overline{\mathcal{C}}_{\bar{\eta}, 1 a}\right\} \cap\left\{\Delta \bar{H}_{1,2}=U\right\},
\end{aligned}
$$

the process $\left(\widetilde{\eta}_{t}\right)_{t \geqslant 0}$ exits from $\overline{\mathcal{C}}_{\bar{\eta}}$ by visiting $\overline{\mathcal{C}}_{\bar{\eta}}\left(m_{0}\right)$ and then leaving it by contraction. So we have the estimate 


$$
\begin{aligned}
& \mathbb{P}\left(\mathbf{A}_{\tilde{\eta}}^{m} \cap\left\{\bar{\eta}_{1} \in \overline{\mathcal{C}}_{\bar{\eta}, 0}\right\} \cap\left\{\Delta \bar{H}_{1,2}=2 U\right\}\right)+\mathrm{P}\left(\mathbf{A}_{\tilde{\eta}}^{m} \cap\left\{\bar{\eta}_{1} \in \overline{\mathcal{C}}_{\bar{\eta}, 1 a}\right\} \cap\left\{\Delta \bar{H}_{1,2}=U\right\}\right) \\
& \leqslant \sum_{i=1}^{e^{\delta_{3} \beta}} \sum_{y \in \overline{\mathcal{C}}_{\bar{\eta}}\left(m_{0}\right)} \mathbb{P}\left(\left\{\zeta_{i}\left(m_{0}\right)<T_{3} e^{\delta_{1} \beta} \wedge \tau_{\overline{\mathcal{C}}_{\bar{\eta}}^{c}}\right\} \cap\left\{\widetilde{\eta}_{\zeta_{i}}=y\right\}\right) \\
& \quad \times \mathbb{P}_{y}\left(\left(\widetilde{\eta}_{t}\right)_{t \geqslant 0} \text { exits by contraction from } \overline{\mathcal{C}}_{\bar{\eta}}\left(m_{0}\right)\right) \\
& \quad+\mathbb{P}\left(\max \left\{i \geqslant 1: \zeta_{i}<T_{3} e^{\delta_{1} \beta} \wedge \tau_{\overline{\mathcal{C}}^{c}}^{\bar{c}_{\bar{\eta}}}\right\}>e^{\delta_{3} \beta}\right) .
\end{aligned}
$$

(3) By using the coupling between $\left(\tilde{\eta}_{t}\right)_{t \geqslant 0}$ and $\left(\bar{\eta}_{t}\right)_{t \geqslant 0}$ we obtain

$$
\begin{aligned}
& \sup _{y \in \mathcal{C}_{\bar{\eta}}\left(m_{0}\right)} \mathbb{P}_{y}\left(\left(\widetilde{\eta}_{t}\right)_{\tau \geqslant 0} \text { exits by contraction from } \overline{\mathcal{C}}_{\bar{\eta}}\left(m_{0}\right)\right) \\
& \leqslant \sup _{y \in \overline{\mathcal{C}}_{\tilde{\eta}}\left(m_{0}\right)} \mathbb{P}_{y}\left(\left(\bar{\eta}_{t}\right)_{t \geqslant 0} \text { exits by contraction from } \overline{\mathcal{C}}_{\tilde{\eta}}\left(m_{0}\right)\right) .
\end{aligned}
$$

On the other hand, by using reversibility we can estimate, for all $\delta>0$,

$$
\mathbb{P}_{y}\left(\left(\widetilde{\eta}_{t}\right)_{t \geqslant 0} \text { exits by contraction from } \overline{\mathcal{C}}_{\bar{\eta}}\left(m_{0}\right)\right) \leqslant e^{-(2 U-\Delta-\delta) \beta} \text {. }
$$

Moreover,

$$
\mathbb{P}\left(\zeta_{i}\left(m_{0}\right)<T_{3} e^{\delta_{1} \beta} \wedge \tau_{\overline{\mathcal{C}}_{\bar{\eta}}^{c}}\right) \leqslant \mathbb{P}\left(\tau_{\overline{\mathcal{C}}_{\tilde{\eta}}\left(m_{0}\right)}<T_{3} e^{\delta_{1} \beta} \wedge \tau_{\overline{\mathcal{C}}_{\bar{\eta}}^{c}}\right),
$$

and thus we obtain

$$
\begin{aligned}
\text { rhs of of }(7.128) \leqslant & e^{\delta_{3} \beta} \mathbb{P}\left(\tau_{\overline{\mathcal{C}}_{\bar{\eta}}\left(m_{0}\right)}<T_{3} e^{\delta_{1} \beta} \wedge \tau_{\mathcal{C}_{\bar{\eta}}^{c}}\right) e^{-(2 U-\Delta-\delta) \beta} \\
& +\mathbb{P}\left(\max \left\{i \geqslant 1: \zeta_{i}<T_{3} e^{\delta_{1} \beta} \wedge \tau_{\mathcal{C}_{\bar{\eta}}^{c}}\right\}>e^{\delta_{3} \beta}\right)
\end{aligned}
$$

(4) Each entrance into the set $\overline{\mathcal{C}}_{\bar{\eta}}\left(m_{0}\right)$, i.e., each $\zeta_{i}\left(m_{0}\right)$, is a time at which a particle exits from $\bar{\Lambda}$ and so it is the endpoint of an interval of type 1b. So, by Lemma 7.91, we have that the last term in (7.132) is SES. The proof of the lemma can now be concluded by iteration after we note that on the event $\left\{\tau_{\overline{\mathcal{C}}_{\bar{\eta}}\left(m_{0}\right)}<T_{3} e^{\delta_{1} \beta} \wedge \tau_{\overline{\mathcal{C}}}^{\bar{\eta}}\right\}$ the process $\left(\widetilde{\eta}_{t}\right)_{t \leqslant 0}$ exits from the set $\overline{\mathcal{C}}_{\bar{\eta}}\left(>m_{0}\right)$ by visiting $\overline{\mathcal{C}}_{\bar{\eta}}\left(m_{0}+1\right)$ and then leaving it by contraction. QED

\subsection{Additional results}

We close by collecting two consequences of Proposition 7.9 that will be needed in Sec. 8. The first concerns the creation of a $2 \times 2$ droplet from $\square$, which is the start of the nucleation path.

Proposition 7.133: There exists $\delta_{6}=\delta_{6}\left(\delta_{1}, \gamma\right)>0$, satisfying $\lim _{\delta_{1}, \gamma \downarrow 0} \delta_{6}\left(\delta_{1}, \gamma\right)=0$, and $\beta_{0}$ $>0$ such that, for all $\beta>\beta_{0}$,

$$
\min _{\eta \in \square \cap \mathcal{X}_{3}} \mathbb{P}_{\eta}\left(\tau_{\mathcal{R}_{2,2}}<e^{(4 \Delta-2 U) \beta+\delta_{6} \beta}\right)>e^{-\delta_{6} \beta} .
$$

Proof: We need the following lemma.

Lemma 7.135: Let $\mathcal{N}_{n}=\left\{\eta \in \mathcal{X}_{0}: N_{\Lambda}(\eta)=n\right\}$. Then there exists $\delta_{7}=\delta_{7}\left(\delta_{1}, \gamma\right)>0$, satisfying $\lim _{\delta 1, \gamma \downarrow 0} \delta_{7}\left(\delta_{1}, \gamma\right)=0$, and $\beta_{0}>0$ such that, for all $\beta>\beta_{0}$, 


$$
\min _{\eta \in \mathcal{N}_{\leqslant 3}} \mathbb{P}_{\eta}\left(\tau_{\mathcal{N}_{4}}<T_{3} e^{2 \delta_{1} \beta}\right) \geqslant e^{-(3 \Delta-2 U) \beta-\delta_{7} \beta}
$$

This result implies Proposition 7.133 as follows. For every $\eta \in \mathcal{N}_{4}$ we have $\mathbb{P}_{\eta}\left(\tau_{\mathcal{R}_{2,2}}<T_{2} e^{\delta_{1} \beta}\right)$ $>e^{-\delta \beta}$ for any $\delta>0$ and $\beta$ large enough, and so for all $\eta \in \square \cap \mathcal{X}_{3}$,

$$
\mathbb{P}_{\eta}\left(\tau_{\mathcal{R}_{2,2}}<e^{(4 \Delta-2 U) \beta+\delta_{6} \beta}\right) \geqslant \mathbb{P}_{\eta}\left(\tau_{\mathcal{N}_{4}}<e^{(4 \Delta-2 U) \beta+\delta^{\prime} \beta}\right) e^{-\delta \beta} \quad \forall 0<\delta^{\prime}<\delta_{6}
$$

and the probability on the rhs of (7.137) is 1 -SES if $\delta^{\prime}>2 \delta_{1}+\delta_{7}$. Indeed, if $\tau_{\mathcal{N}_{4}}$ $\geqslant e^{(4 \Delta-2 U) \beta+\delta^{\prime} \beta}$, then in any subinterval of length $T_{3} e^{2 \delta_{1} \beta}$ contained in the interval [0, $e^{(4 \Delta-2 U) \beta+\delta^{\prime} \beta}$ ] we have not yet reached $\mathcal{N}_{4}$. By (7.136), the probability of this event is SES if $\delta^{\prime}>2 \delta_{1}+\delta_{7} . \quad$ QED

Proof of Lemma 7.135:

(1) By using the recurrence to $\mathcal{X}_{3}$ within time $T_{3} e^{\delta_{1} \beta}$, we have, for all $\eta \in \mathcal{N}_{\leqslant 3}$,

$$
\begin{aligned}
\mathbb{P}_{\eta}\left(\tau_{\mathcal{N}_{4}}<T_{3} e^{2 \delta_{1} \beta}\right) & \geqslant \mathbb{P}_{\eta}\left(\left\{\tau_{\mathcal{N}_{4}}<T_{3} e^{2 \delta_{1} \beta}\right\} \cap\left\{\tau_{\mathcal{N}_{4}}>\tau_{\mathcal{X}_{3}}\right\}\right) \\
& \geqslant \min _{\eta^{\prime} \in \square \cap \mathcal{X}_{3}} \mathbb{P}_{\eta^{\prime}}\left(\tau_{\mathcal{N}_{4}}<T_{3} e^{2 \delta_{1} \beta}-T_{3} e^{\delta_{1} \beta}\right) \mathbb{P}_{\eta}\left(\tau_{\mathcal{N}_{4}}>T_{3} e^{\delta_{1} \beta}\right)-\mathrm{SES}
\end{aligned}
$$

since $\mathcal{N}_{\leqslant 3} \cap \mathcal{X}_{3}=\square \cap \mathcal{X}_{0}$.

(2) Let

$$
\mathcal{A}=\left\{\eta \in \mathcal{X}_{0}: N_{\Lambda_{l_{-}} \backslash \bar{\Lambda}}(\eta) \geqslant 4\right\}
$$

Then, for all $\eta \in \square \cap \mathcal{X}_{3}$ and $\beta$ sufficiently large,

$$
\begin{aligned}
\mathbb{P}_{\eta}\left(\tau_{\mathcal{N}_{4}}<\right. & \left.T_{3} e^{2 \delta_{1} \beta}-T_{3} e^{\delta_{1} \beta}\right) \geqslant \mathbb{P}_{\eta}\left(\left\{\tau_{\mathcal{A}}<\frac{1}{3} T_{3} e^{2 \delta_{1} \beta}\right\} \cap\left\{\tau_{\mathcal{N}_{\geqslant 2}}>\frac{1}{3} T_{3} e^{2 \delta_{1} \beta}\right\}\right) \\
& \times \min _{\eta^{\prime} \in \mathcal{A} \cap \mathcal{N}_{<2}} \mathbb{P}_{\eta^{\prime}}\left(\tau_{\mathcal{N}_{4}}<T_{3} e^{-\gamma / 2 \beta}\right) .
\end{aligned}
$$

The first probability on the rhs of (7.140) can be estimated by recalling (2.27), $\left|\Lambda_{l_{-}}\right|=e^{(\Delta-\gamma) \beta}$ and $\left|\Lambda_{l_{+}}\right|=e^{(\Delta+\delta) \beta}$, namely, for all $\eta \in \square \cap \mathcal{X}_{3}$ :

$$
\begin{aligned}
& \mathbb{P}_{\eta}\left(\left\{\tau_{\mathcal{A}}<\frac{1}{3} T_{3} e^{2 \delta_{1} \beta}\right\} \cap\left\{\tau_{\mathcal{N}_{\geqslant 2}}>\frac{1}{3} T_{3} e^{2 \delta_{1} \beta}\right\}\right) \\
& \geqslant\left(\frac{\left|\Lambda_{l_{-}} \backslash \bar{\Lambda}\right|}{\frac{1}{3} T_{3} e^{2 \delta_{1} \beta}}\right)^{4}\left(1-\mathbb{P}_{\eta}\left(\tau_{\mathcal{N}_{\geqslant}} \leqslant \frac{1}{3} T_{3} e^{2 \delta_{1} \beta}\right)\right) \\
& \geqslant e^{-\left(\gamma+2 \delta_{1}\right) 4 \beta}\left(1-\frac{|\bar{\Lambda}|^{2}}{\frac{1}{2} T_{3} e^{2 \delta_{1} \beta}}\right) \geqslant e^{-\left(\delta_{7} / 10\right) \beta} .
\end{aligned}
$$

The second probability on the rhs of (7.140) can be estimated by similar standard estimates on SRW. Indeed, the fact that $\eta^{\prime} \in \mathcal{A}$ allows us to estimate from below by $e^{-\Delta \beta-\left(\delta_{7} / 10\right) \beta}$ the probability that a particle arrives in $\bar{\Lambda}$ at time $t$, provided $t>\frac{1}{3} T_{3} e^{-(\gamma / 2) \beta}$. If we denote by $\tau_{1}^{\prime}, \tau_{2}^{\prime}, \tau_{3}^{\prime}, \tau_{4}^{\prime}$, the random times corresponding to the arrival of the first four particles, then we obtain 


$$
\begin{aligned}
& \min _{\eta^{\prime} \in \mathcal{A} \cap \mathcal{N}_{<2}} \mathrm{P}_{\eta}\left(\tau_{\mathcal{N}_{4}}<T_{3} e^{-(\gamma / 2) \beta}\right) \\
& \geqslant \min _{\eta^{\prime} \in \mathcal{A} \cap \mathcal{N}_{<2}} \sum_{t=\frac{1}{3} T_{3} e^{-\delta^{\prime} \beta}}^{\frac{2}{3} T_{3} e^{-\delta^{\prime} \beta}} \sum_{t_{3}=t+2}^{t+e^{U \beta-\left(\delta_{7} / 10\right) \beta}} \sum_{t_{3}+e^{U \beta-\left(\delta_{7} / 10\right) \beta}} \\
& \mathbb{P}_{\eta^{\prime}}\left(\tau_{1}^{\prime}=t, \tau_{2}^{\prime}=t+1, \tau_{3}^{\prime}=t_{3}, t_{4}^{\prime}=t_{4}, \mathbf{D}_{2}, \mathbf{D}_{3}\right) \\
& \geqslant e^{-(3 \Delta-2 U) \beta-\left(9 \delta_{7} / 10\right) \beta},
\end{aligned}
$$

where $\mathbf{D}_{2}$ is the event that the first two particles form and stay a dimer until time $e^{U \beta-\left(\delta_{7} / 10\right) \beta}$ and $\mathbf{D}_{3}$ the same for a trimer. QED

The second consequence of Proposition 7.9 concerns some estimates for the transition probabilities of the reduced Markov chain. Let $\tau_{i}, i \in \mathbb{N}_{0}$ be as in Definition 7.3 and let $\bar{\tau}_{1}$ $=\min \left\{\tau_{i}: \eta_{\tau_{i}} \notin \mathcal{E}_{3}\left(\eta_{\tau_{0}}\right)\right\}$, i.e., the first time $\tau_{i}$ at which the reduced Markov chain changes configuration.

Proposition 7.143: Let $\delta$ be as in Proposition 7.9.

(i) If $\bar{\eta} \in \mathcal{R}_{l_{1}, l_{2}}$ with $l_{1}<l_{c}$ and $\bar{\eta}^{\prime} \in \mathcal{R}_{l_{1}+1, l_{2}}$, then

$$
\min _{\eta \in I_{\bar{\eta}}} \mathbb{P}_{\eta}\left(\left\{\eta_{\bar{\tau}_{1}} \in I_{\bar{\eta}^{\prime}}\right\} \cap\left\{\bar{\tau}_{1}<e^{(2 \Delta-U+\delta) \beta}\right\}\right) \geqslant e^{-[\Delta-U-r(\bar{\eta})+2 \delta] \beta} .
$$

(ii) If $\bar{\eta} \in \mathcal{R}_{l_{1}, l_{2}}$ with $l_{1} \geqslant l_{c}$ and $\bar{\eta}^{\prime} \in \mathcal{R}_{l_{1}+1, l_{2}}$, then

$$
\min _{\eta \in I_{\bar{\eta}}} \mathbb{P}_{\eta}\left(\left\{\eta_{\bar{\tau}_{1}} \in I_{\bar{\eta}^{\prime}}\right\} \cap\left\{\bar{\tau}_{1}<e^{(2 \Delta-U+\delta) \beta}\right\}\right) \geqslant e^{-2 \delta \beta} .
$$

(iii) If $\bar{\eta}$ is a lacunary set and $\bar{\eta}_{j}, j=0,1, \ldots, n(n \in \mathbb{N})$, is the sequence of configurations defined in Proposition 7.9 (iii), then

$$
\min _{j=0, \ldots, n-1} \min _{\eta_{j} \in I_{\bar{\eta}_{j}}} \mathbb{P}_{\eta_{j}}\left(\left\{\eta_{\bar{\tau}_{1}} \in I_{\bar{\eta}_{j+1}}\right\} \cap\left\{\bar{\tau}_{1}<e^{(2 \Delta-U+\delta) \beta}\right\}\right) \geqslant e^{-2 \delta \beta} .
$$

Proof: (i) Estimate

$$
\mathbb{P}_{\eta}\left(\eta_{\bar{\tau}_{1}} \in I_{\bar{\eta}^{\prime}}\right)=\frac{P^{R}\left(\eta, I_{\bar{\eta}^{\prime}}\right)}{1-P^{R}\left(\eta, I_{\bar{\eta}}\right)} \geqslant \frac{P^{R}\left(\eta, I_{\bar{\eta}^{\prime}}\right)}{C(|\bar{\Lambda}|) \max _{\eta \in I_{\bar{\eta}} \eta^{\prime \prime} \in \mathcal{X}_{3}, \eta^{\prime \prime} \notin \mathcal{E}_{3}(\bar{\eta})} P^{R}\left(\eta, I_{\bar{\eta}^{\prime \prime}}\right)},
$$

where $C(|\bar{\Lambda}|)>0$ is some constant depending only on $|\bar{\Lambda}|$. By Proposition 7.9 (i) and (iv), we have

$$
\mathbb{P}_{\eta}\left(\eta_{\bar{\tau}_{1}} \in I_{\bar{\eta}^{\prime}}\right) \geqslant e^{-[\Delta-U-r(\bar{\eta})+2 \delta] \beta} .
$$

By Proposition 7.9(i), we also have

$$
\begin{aligned}
\mathbb{P}_{\eta}\left(\bar{\tau}_{1} \geqslant e^{(2 \Delta-U) \beta+\delta \beta}\right) & \leqslant \mathrm{SES}+\left[P^{R}\left(\eta, I_{\bar{\eta}}\right)\right]^{\left[e^{(2 \Delta-U+\delta) \beta} / T_{3} e^{\delta_{1} \beta}\right]} \\
& =\mathrm{SES}+\left[1-\sum_{\bar{\eta}^{\prime \prime} \notin \mathcal{E}_{3}(\bar{\eta})} P^{R}\left(\eta, I_{\bar{\eta}^{\prime \prime}}\right)\right]^{\left[e^{(2 \Delta-U+\delta) \beta} / T_{3} e^{\delta_{1} \beta}\right]} \\
& \leqslant \mathrm{SES}+\left[1-P^{R}\left(\eta, I_{\bar{\eta}^{\prime}}\right)\right]^{\left[e^{(2 \Delta-U+\delta) \beta} / T_{3} e^{\delta_{1} \beta}\right]}=\mathrm{SES} .
\end{aligned}
$$


Parts (ii) and (iii) follow in the same way. QED

\section{PROOF OF THE MAIN THEOREM}

In this section we collect the results from Secs. 4-7 and prove Theorem 1.53.

\subsection{Lower bound for the nucleation time}

We begin by proving the lower bound in Theorem 1.53(c), i.e.,

$$
\lim _{\beta \rightarrow \infty} \mathbb{P}_{\nu_{\square}}\left(\tau_{\square}<T_{-}\right)=0 \quad \forall \delta>0 \quad \text { with } T_{-}=T_{-}(\beta, \delta)=e^{(\Gamma-\delta) \beta} .
$$

(1) Let

$$
\mathcal{A}=\left\{\eta^{\prime} \in \mathcal{X}: \bar{H}\left(\square, \eta^{\prime}\right)-\bar{H}(\square)<\Gamma\right\}
$$

where $\bar{H}$ is the local grand-canonical Hamiltonian defined in (1.49), the communication height $\bar{H}\left(\square, \eta^{\prime}\right)$ is the obvious extension of $(4.12)$, and $\bar{H}(\square)=0$.

Since $\Gamma$ is the communication height between $\square$ and $\boldsymbol{\square}$, as was shown in Proposition 4.24 (iii), we have $\tau_{\partial \mathcal{A}}<\tau_{\square}$ and so

$$
\mathrm{P}_{\nu_{\bar{\square}}}\left(\tau_{\mathbf{\square}}<T_{-}\right) \leqslant \mathbb{P}_{\nu_{\bar{\square}}}\left(\tau_{\partial \mathcal{A}}<T_{-}\right) .
$$

(2) To estimate the rhs of (8.3) we use reversibility. For that it is convenient to pass to the discrete-time setup. Let $T_{i}, i \in \mathbb{N}_{0}$, be the successive times at which some clock in $\bar{\Lambda} * \cup \partial \bar{\Lambda} *$ rings. Let $P^{*}\left(\eta, \eta^{\prime}\right)$ denote the transition probabilities of the Markov chain that is obtained by observing our process at these times:

$$
P^{*}\left(\eta, \eta^{\prime}\right)=\mathbb{P}_{\eta}\left(\eta_{T_{1}}=\eta^{\prime}\right)
$$

Let

$$
\begin{gathered}
i^{*}=\inf \left\{i \in \mathbb{N}_{0}: \eta_{T_{i}} \in \partial \mathcal{A}\right\}, \\
N_{1}=\#\left\{0 \leqslant i<i^{*}: T_{i+1}-T_{i}<a\right\}, \\
N_{2}=\#\left\{0 \leqslant i<i^{*}: T_{i+1}-T_{i} \geqslant a\right\},
\end{gathered}
$$

where $a>0$ is a constant that will be chosen shortly. Since $i^{*}=N_{1}+N_{2}$ and $\left\{\tau_{\partial \mathcal{A}}<T_{-}\right\} \subset\left\{N_{2}\right.$ $\left.<T_{-} / a\right\}$, we have, for any $M \in \mathbb{N}$,

$$
\mathbb{P}_{\nu_{\square}}\left(\tau_{\partial \mathcal{A}}<T_{-}\right) \leqslant \mathrm{P}\left(N_{1}>M, N_{2}<T_{-} / a\right)+\mathbb{P}_{\nu_{\square}}\left(i^{*}<M+T_{-} / a\right) .
$$

Moreover,

$$
\mathbb{P}\left(N_{1}>M, N_{2}<T_{-} / a\right) \leqslant \mathrm{P}\left(\frac{N_{1}}{N_{1}+N_{2}} \geqslant \frac{M}{M+T_{-} / a}\right) .
$$

Now pick $a, M$ such that

$$
\mathrm{P}\left(T_{1}<a\right) \leqslant \frac{1}{3}, \quad M=T_{-} / a .
$$

Then the probability on the rhs of (8.7) equals the probability that among the first $N_{1}+N_{2}$ of a sequence of Bernoulli trials with success probability $\leqslant \frac{1}{3}$ a fraction at least $\frac{1}{2}$ is successful. However, this probability is SES, and so we get 


$$
\mathrm{P}_{\nu_{\square}}\left(\tau_{\partial, \mathcal{A}}<T_{-}\right)=\mathrm{SES}+\mathrm{P}_{\nu_{\square}}\left(i^{*}<2 T_{-} / a\right) .
$$

(3) Let us next consider the Markov chain obtained from our process when some clock in the large volume $\Lambda_{\beta}$ rings. Let $\hat{P}\left(\eta, \eta^{\prime}\right)$ denote its transition probabilities. This Markov chain is easily seen to be reversible w.r.t. the same invariant measure $\nu$ as the continuous-time process. From this observation we deduce the reversibility of the Markov chain with transition probabilities given by $P^{*}\left(\eta, \eta^{\prime}\right)$ in (8.4). Indeed,

$$
\begin{aligned}
P^{*}\left(\eta, \eta^{\prime}\right) & =\hat{P}\left(\eta, \eta^{\prime}\right)+\sum_{t=2}^{\infty} \sum_{\substack{\eta_{1}, \ldots,\left.\eta_{t-1} \\
\eta_{i}\right|_{\Lambda}=\left.\eta\right|_{\Lambda} ^{-}(i=1, \ldots, t-1)}} \hat{P}\left(\eta, \eta_{1}\right) \times \cdots \times \hat{P}\left(\eta_{t-1}, \eta^{\prime}\right) \\
& =\frac{\nu(\eta)}{\nu(\eta)}\left[\hat{P}\left(\eta^{\prime}, \eta\right)+\sum_{t=2}^{\infty} \sum_{\substack{\eta_{1}, \ldots,\left.\eta_{t-1} \\
\eta_{i}\right|_{\bar{\Lambda}}=\left.\eta\right|_{\Lambda}(i=1, \ldots, t-1)}} \hat{P}\left(\eta^{\prime}, \eta_{t-1}\right) \times \cdots \times \hat{P}\left(\eta_{1}, \eta\right)\right] \\
& =\frac{\nu(\eta)}{\nu\left(\eta^{\prime}\right)} P^{*}\left(\eta^{\prime}, \eta\right) .
\end{aligned}
$$

Hence we get

$$
\begin{aligned}
\mathbb{P}_{\nu_{\square}}^{*}\left(i^{*}\right. & \left.<2 T_{-} / a\right)=\frac{1}{\nu\left(I_{\square}\right)} \sum_{l=1}^{2 T_{-} / a} \sum_{\substack{\eta_{1}, \eta_{2}, \ldots, \eta_{l-1} \in \mathcal{A} \\
\eta_{l} \in \partial \mathcal{A}}} P^{*}\left(\eta_{1}, \eta_{2}\right) \times \cdots \times P^{*}\left(\eta_{l-1}, \eta_{l}\right) \\
& \leqslant \frac{2 T_{-}}{a} \sup _{\xi \in \partial \mathcal{A}} \frac{\nu(\xi)}{\nu\left(I_{\square}\right)} .
\end{aligned}
$$

From (8.2), (8.9), (8.11), Proposition 4.24(iii), and Proposition A6 we get the result. Namely, we replace $\nu$ by $\mu$, making an error that is SES because $\lim _{\beta \rightarrow \infty}(1 / \beta) \log \left|\Lambda_{\beta}\right|=\infty$, and we use that $\sup _{\xi \in \mathcal{H A}} \mu(\xi) / \mu\left(I_{\square} \bar{\square}\right) \leqslant e^{-\Gamma \beta}$ because of (8.2) [recall (4.10)].

\subsection{Upper bound for the nucleation time}

Next we prove the upper bound in Theorem 1.53(c), i.e.,

$$
\lim _{\beta \rightarrow \infty} \mathbb{P}_{\nu_{\square}}\left(\tau_{\mathbf{\square}}>T_{+}\right)=0 \quad \forall \delta>0 \quad \text { with } T_{+}=T_{+}(\beta, \delta)=e^{\beta(\Gamma+\delta)} .
$$

(1) The idea is to construct an event $\mathbf{E}_{\eta, T}$ leading from any $\eta \in \mathcal{X}$ to $\mathbf{\square}$ in an appropriate time $T=T(\beta, \delta)$ and having a sufficiently large probability. Let us first describe this event in words. The time $T$ will be chosen of the form $T=e^{[4 \Delta-2 U+(\delta / 2)] \beta}$ for a suitably small $\delta>0$. Note that, for $\beta$ sufficiently large, $T / 3>T_{3}^{+}$with $T_{3}^{+}=e^{[\Delta+(\delta / 2)] \beta}$. But, given $\eta \in I_{\square}^{-}$, we know from Proposition 5.20 that within time $T_{3}^{+}$our process visits $\overline{\mathcal{X}}_{3}$ with a large probability. Then, by Proposition 5.11 (iii) and Remark (2) following it, either of the two following situations prevails:

(1) There exist $1, l^{\prime} \in \mathbb{N}_{0} \backslash\{1\},\left|l-l^{\prime}\right| \leqslant 1$ such that our process visits $\mathcal{R}_{l, l^{\prime}}$ within time $T_{3}^{+}$.

(2) The process passes through a configuration containing a large lacunary square or quasi-square [recall Remark (2) in Sec. 5.2].

In case (2), within another time $T / 3$ with a large probability our process goes to a large (highly supercritical) square or quasi-square. Indeed, it follows from the results in Sec. 7 that for $\beta$ sufficiently large this happens within a time $e^{[2 U+(\delta / 2)] \beta}$ for any $\delta>0$. But for $\delta>0$ small we have $T / 3>e^{[2 U+(\delta / 2)] \beta}$. 
From the square or quasi-square present in each configuration in $\mathcal{R}_{l, l^{\prime}}$, we grow following a sequence of squares or quasi-squares of increasing side lengths, leading eventually to $\boldsymbol{\square}$. Of course, it may happen that the initial square or quasi-square is empty $\left(l=l^{\prime}=0\right)$, i.e., the configuration on which we fall during the first part of our event is just $\bar{\square}$.

(2) Let us now give precise definitions. We set

$$
T_{3}^{+}=e^{[\Delta+(\delta / 2)]}, \quad T_{4}^{+}=e^{[2 U+(\delta / 2)] \beta} .
$$

For $\eta \in \mathcal{X}$, define the set of trajectories

$$
\mathbf{E}_{\eta, T_{3}^{+}}^{(1)}=\left\{\phi: \phi_{0}=\eta, \exists 0 \leqslant t \leqslant T_{3}^{+}: \phi_{t} \in \mathcal{X}_{3}\right\} .
$$

Let $\mathcal{Q}$ be the set of square or quasi-square configurations:

$$
\mathcal{Q}=\cup_{l, l^{\prime} \neq 1:\left|l-l^{\prime}\right| \leqslant 1} \mathcal{R}_{l, l^{\prime}},
$$

and recall that $\mathcal{Q}=\mathcal{X}_{3} \backslash \mathcal{L}$. For $\eta \in \mathcal{X}_{3}$, define the set of trajectories

$$
\mathbf{E}_{\eta, T_{4}^{+}}^{(2)}=\left\{\phi: \phi_{0}=\eta, \exists 0 \leqslant t \leqslant T_{4}^{+}: \phi_{t} \in \mathcal{Q}\right\}
$$

and put

$$
\bar{\tau}_{2}=\bar{\tau}_{2}(\phi)=\inf \left\{t \geqslant 0: \phi_{t} \in \mathcal{Q}\right\} .
$$

Note that for any $\eta \in \mathcal{X}_{3} \backslash \mathcal{Q}$ the interval $\bar{\tau}_{2}-\tau_{\mathcal{X}_{3}}$ is strictly positive, while for any $\eta \in \mathcal{X}_{3} \cap \mathcal{R}_{l, l^{\prime}}$ with $1, l^{\prime} \in \mathbb{N}\{1\},\left|l-l^{\prime}\right| \leqslant 1$ it is zero and the corresponding set $\mathbf{E}_{\eta, T_{4}^{+}}^{(2)}$ is trivial.

(3) Given $l_{1}, l_{2} \in \mathbb{N}_{0} \backslash\{1\},\left|l_{1}-l_{2}\right| \leqslant 1$, we next introduce $\mathbf{E}_{R_{l_{1}, l_{2}, T}}^{(3)}$ as the set of trajectories starting from $\mathcal{R}_{l_{1}, l_{2}}$ and passing (at the successive times $\tau_{1}, \tau_{2}, \ldots$ of return to $\mathcal{X}_{3}$ to a different configuration in $\bar{\Lambda}$ ) through the following sequence $\psi=\psi_{1}, \psi_{2}, \ldots$ of pairs of integers:

$$
\begin{array}{ll}
\left(l_{1}, l_{2}\right)=(l, l), l \geqslant 2: & \psi=(l, l),(l+1, l),(l+1, l+1), \ldots,\left(l_{0}, l_{0}\right) . \\
\left(l_{1}, l_{2}\right)=(l, l+1), l \geqslant 2: & \psi=(l, l+1),(l+1, l+1),(l+1, l+2), \ldots,\left(l_{0}, l_{0}\right) . \\
\left(l_{1}, l_{2}\right)=(0,0): & \psi=(0,0),(2,2),(2,3),(3,3), \ldots,\left(l_{0}, l_{0}\right) .
\end{array}
$$

More precisely, put $\tau_{0}=0$ and, for $i \in \mathbb{N}$,

$$
\tau_{i}=\min \left\{t>\tau_{i-1}: \eta_{t} \in \mathcal{X}_{3} \backslash I_{\bar{\eta}_{t}}\right\},
$$

where $\bar{\eta}_{t}=\left.\eta_{t}\right|_{\bar{\Lambda}}$. (The $\tau_{i}$ are the random times at which the reduced Markov chain changes configuration inside $\bar{\Lambda}$; see Definition 7.3.) Let $\mathcal{R}_{\psi}$ be the sequence of sets of configurations $\mathcal{R}_{l, l^{\prime}}$ with $\left(l, l^{\prime}\right)$ following the sequence $\psi$. Then we define

$$
\mathbf{E}_{R_{l_{1}, l_{2}, T}^{(3)}}^{(3)}=\left\{\phi: \phi_{\tau_{0}} \in \mathcal{R}_{l_{1}, l_{2}}, \phi_{\tau_{i}} \in \mathcal{R}_{\psi_{i}} \forall i \in \mathbb{N}, \max _{i}\left(\tau_{i+1}-\tau_{i}\right)<e^{[4 \Delta-2 U+(\delta / 2)] \beta}\right\} .
$$

(4) Our event $\mathbf{E}_{\eta, T}$ can now be defined as

$$
\mathbf{E}_{\eta, T}=\bigcup_{\eta_{1} \in \mathcal{X}_{3}, \eta_{2} \in \mathcal{Q}}\left\{\mathbf{E}_{\eta, T_{3}^{+}}^{(1)} \cap\left\{\eta_{\tau_{1}}=\eta_{1}\right\}\right\} \cap\left\{\mathbf{E}_{\eta_{1}, T_{4}^{+}}^{(2)} \cap\left\{\eta_{\bar{\tau}_{2}}=\eta_{2}\right\}\right\} \cap \mathbf{E}_{\eta_{2}, T}^{(3)} .
$$

We will estimate the probability of each of the parts.

(5) We have, for $\beta$ sufficiently large, 


$$
\inf _{l_{1}, l_{2}} \inf _{\eta \in \mathcal{R}_{l_{1}, l_{2}}} \mathbb{P}_{\eta}\left(\mathbf{E}_{\eta, T}^{(3)}\right) \geqslant e^{-[\Gamma-4 \Delta+2 U-(\delta / 4)] \beta} .
$$

Indeed, suppose first that $\left(l_{1}, l_{2}\right)=(0,0)$. Then it follows from Proposition 7.133 and 7.143 that

$$
\begin{gathered}
\mathrm{P}_{\nu \bar{\square}}\left(\tau_{1} \in \mathcal{R}_{2,2}, \tau_{1}<e^{-(4 \Delta-2 U+\delta / 2) \beta}\right) \asymp 1, \\
\min _{\eta \in \psi_{i}} \mathbb{P}_{\psi_{i}}\left(\eta_{\tau_{1}} \in \mathcal{R}_{\psi_{i+1}}, \tau_{1}<e^{\left(r_{i}+\Delta+\delta / 2\right) \beta}\right) \asymp e^{-\left(\Delta-U-r_{i}\right) \beta},
\end{gathered}
$$

where $\asymp$ denotes logarithmic equivalence in $\beta$ and, for $\psi_{i}=(l, l+1)$ or $\psi_{i}=(l, l)$ with $l<l_{c}$, we put $r_{i}=(2 U-\Delta)(l-1)$. If, on the other hand, $l \geqslant l_{c}$, then we have by Proposition 7.143 that

$$
\mathbb{P}_{\psi_{i}}\left(\eta_{\tau_{1}} \in \mathcal{R}_{\psi_{i+1}}, \tau_{1}<e^{(2 \Delta-U+\delta / 2) \beta}\right) \asymp 1 .
$$

The case $\left(l_{1}, l_{2}\right) \neq(0,0)$ can be treated in a similar way: it turns out that the worst lower estimate corresponds to the case $\left(l_{1}, l_{2}\right)=(0,0)$. Equation (8.21) follows from an immediate computation.

(6) It follows from Propositions 6.2 and 7.9 that, for all $\eta \in \mathcal{X}$ and $\eta_{1} \in \mathcal{X}_{3}$,

$$
\mathbb{P}\left(\mathbf{E}_{\eta, T_{3}^{+}}^{(1)}\right) \asymp 1, \quad \mathbb{P}\left(\mathbf{E}_{\eta_{1}, T_{4}^{+}}^{(2)}\right) \asymp 1 .
$$

Thus, from (8.20), (8.21), and (8.24) we get

$$
\mathrm{P}\left(\mathbf{E}_{\eta, T}\right) \geqslant e^{-[\Gamma-4 \Delta+2 U+(\delta / 2)] \beta} .
$$

We can now apply Proposition 2.13 with

$$
\begin{gathered}
\nu=\nu_{\bar{\square}}, \\
T=e^{[4 \Delta-2 U+(\delta / 2)] \beta}, \quad T^{\prime}=T^{\prime \prime}=e^{(\Gamma+\delta) \beta}, \\
\mathcal{A}=\mathbf{\square}, \quad \mathcal{B}=\mathcal{X}, \quad p=e^{-[\Gamma-4 \Delta+2 U+(\delta / 4)] \beta},
\end{gathered}
$$

to complete the proof.

\subsection{The gate for the nucleation}

In this section we prove Theorem 1.53 (b).

(1) Abbreviate

$$
N(\eta)=N_{\bar{\Lambda}}(\eta)=\sum_{x \in \bar{\Lambda}} \eta(x)
$$

and consider the sets

$$
\begin{aligned}
& \mathcal{G}=\left\{\eta \in \mathcal{X}: N(\eta)<l_{c}\left(l_{c}-1\right)+2\right\}, \\
& \mathcal{G}^{-}=\left\{\eta \in \mathcal{X}: N(\eta)=l_{c}\left(l_{c}-1\right)+1\right\}, \\
& \mathcal{G}^{0}=\left\{\eta \in \mathcal{X}: N(\eta)=l_{c}\left(l_{c}-1\right)+2\right\} .
\end{aligned}
$$

Given a path $\phi=\phi_{1}, \ldots, \phi_{m}(m \in \mathbb{N})$ with $\phi_{1}=\square, \phi_{m}=\mathbf{\square}$, let $i^{0}=i^{0}(\phi)$ be the first hitting time of $\mathcal{G}^{0}$ :

$$
i^{0}=\inf \left\{i \in \mathbb{N}_{:} \phi_{i} \in \mathcal{G}^{0}\right\}
$$

Then 


$$
\bar{H}\left(\phi_{i 0}\right)=H\left(\phi_{i^{0}-1}\right)+\Delta,
$$

since $\bar{H}$ increases by $\Delta$ when we add a particle to $\bar{\Lambda}$.

(2) We have

$$
\lim _{\beta \rightarrow \infty} \mathbb{P}_{\nu_{\bar{\square}}}\left(\eta_{\tau_{\mathcal{G}^{0}}} \notin \mathcal{D}_{l_{c}-1, l_{c}}^{0}\right)=0 .
$$

Indeed, since $\mathbb{P}_{\nu_{\bar{\square}}}\left(\tau_{\mathcal{G}^{0}}<\tau_{\square}\right)=1$, it follows from Theorem 1.53 (c) that

$$
\lim _{\beta \rightarrow \infty} \mathbb{P}_{\nu_{\bar{\square}}}\left(\tau_{\mathcal{G}^{0}}>e^{(\Gamma+\delta) \beta}\right)=0 \quad \forall \delta>0 .
$$

On the other hand, from the results in Sec. 4.3 we know that

$$
\bar{H}\left(\square, \mathcal{G}^{-} \backslash \mathcal{D}_{l_{c}-1, l_{c}}^{-}\right) \leqslant \bar{H}\left(\mathcal{D}_{l_{c}-1, l_{c}}^{-}\right)+U .
$$

Using (8.33) we can deduce, via an argument based on reversibility similar to the one used to prove $(8.11)$, that

$$
\lim _{\beta \rightarrow \infty} \mathbb{P}_{\nu_{\bar{\square}}}\left(\theta_{\square, \boldsymbol{\varpi}}<\tau_{\mathcal{G}^{-} \backslash \mathcal{D}_{l_{c}-1, l_{c}}^{-}}<e^{(\Gamma+U / 2) \beta}\right)=0 .
$$

(3) The claim now follows from (8.32) and (8.34) after choosing $\delta$ sufficiently small.

\subsection{Criticality for squares and quasi-squares}

In this section we prove Theorem 1.53(a).

(1) For $l_{1}, l_{2}$ with $0 \leqslant l_{1} \leqslant l_{2}$ and $\left|l_{1}-l_{2}\right| \leqslant 1$, let $\mathcal{R}^{>\left(l_{1}, l_{2}\right)}$ denote the set of all configurations whose restriction to $\bar{\Lambda}$ gives rise to a single square or quasi-square strictly larger than $R_{l_{1}, l_{2}}$, i.e.,

$$
\mathcal{R}^{>\left(l_{1}, l_{2}\right)}=\underset{\left(\tilde{\ell}_{1}, \tilde{\ell}_{2}\right)>\left(l_{1}, l_{2}\right)}{\bigcup} \mathcal{R}_{l_{1}, \tilde{l}_{2}},
$$

where $\left(\tilde{l}_{1}, \tilde{l}_{2}\right)>\left(l_{1}, l_{2}\right)$ (with $\left.\tilde{l}_{1} \leqslant \tilde{l}_{2}, l_{1} \leqslant l_{2}\right)$ means either $\tilde{l}_{1}>l_{1}, \tilde{l}_{2} \geqslant l_{2}$ or $\tilde{l}_{1}=l_{1}, \tilde{l}_{2}>l_{2}$. Let

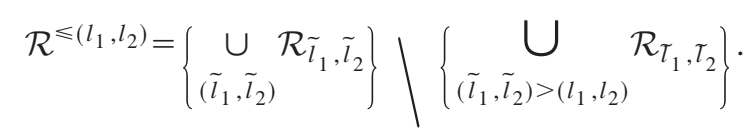

Similarly, we define $\mathcal{R}^{<\left(l_{1}, l_{2}\right)}$. and $\mathcal{R}^{\geqslant\left(l_{1}, l_{2}\right)}$.

(2) Let us first consider the subcritical case. With the help of reversibility, like in (8.11), we can prove that, for every $\delta>0$ and every $l_{1}, l_{2}$ such that $0 \leqslant l_{1} \leqslant l_{2},\left|l_{1}-l_{2}\right| \leqslant 1$, and $l_{1} \leqslant l_{c}$,

$$
\lim _{\beta \rightarrow \infty} \mathbb{P}_{\nu_{R_{l_{1}, l_{2}}}}\left(\tau_{\mathcal{R}}>\left(l_{1}, l_{2}\right)<e^{(2 \Delta-U-\delta) \beta}\right)=0 .
$$

Indeed, it follows from Proposition 4.24 (ii) that the saddle of exit from $\mathcal{R}^{\leqslant\left(l_{1}, l_{2}\right)}$, i.e., the configurations realizing the communication height between $\mathcal{R}^{\leqslant\left(l_{1}, l_{2}\right)}$ and $\mathcal{X}_{3} \backslash \mathcal{R}^{\leqslant\left(l_{1}, l_{2}\right)}$ $=\mathcal{R}^{>\left(l_{1}, l_{2}\right)} \cup \mathcal{L}$ or $\mathcal{R}^{>\left(l_{1}, l_{2}\right)}$, is $2 \Delta-U$.

(3) For $l_{1}, l_{2}$ such that $0 \leqslant l_{1} \leqslant l_{2} \leqslant l_{1}+1$ and $l_{1} \leqslant l_{c}-1$, we define a shrinking event $\mathbf{E}_{l_{1}, l_{2}}^{s}$ containing the set of trajectories starting from $\mathcal{R}_{l_{1}, l_{2}}$ and passing, at the successive times $\tau_{1}, \tau_{2}, \ldots$ defined in (8.18), through the following sequence $\psi^{s}=\psi_{1}^{s}, \psi_{2}^{s}, \ldots$ of pairs of integers:

$$
\left(l_{1}, l_{2}\right)=(l, l), l \geqslant 2: \quad \psi^{s}=(l, l),(l-1, l),(l-1, l-1), \ldots,(0,0),
$$




$$
\begin{array}{ll}
\left(l_{1}, l_{2}\right)=(l, l+1), l \geqslant 2: & \psi^{s}(l, l+1),(l, l),(l-1, l),(l-1, l-1), \ldots,(0,0), \\
\left(l_{1}, l_{2}\right)=(1,2): & \psi^{s}=(1,2),(1,1),(0,0), \\
\left(l_{1}, l_{2}\right)=(1,2): & \psi^{s}=(1,1),(0,0), \\
\left(l_{1}, l_{2}\right)=(0,0): & \psi^{s} \text { is the trivial path with no move. }
\end{array}
$$

More precisely, we denote by $\mathcal{R}_{\psi^{s}}$ the sequence of sets of configurations $\mathcal{R}_{l, l^{\prime}}$ with $\left(l, l^{\prime}\right)$ following the sequence $\psi^{s}$, and define

$$
\mathbf{E}_{l_{1}, l_{2}}^{s}=\left\{\phi: \phi_{\tau_{0}} \in \mathcal{R}_{l_{1} l_{2}}, \phi_{\tau_{i}} \in \mathcal{R}_{\psi_{i}^{s}} \quad \forall i \in \mathbb{N}, \max _{i}\left(\tau_{i+1}-\tau_{i}\right)<e^{\left[(2 U-\Delta)\left(l_{1}-2\right)+2 U+\delta\right] \beta}\right\} .
$$

(4) Let us next consider the supercritical case. Again, with the help of reversibility like in (8.11), we can prove that, for every $\delta>0$ and every $l_{1}, l_{2}$ such that $l_{c} \leqslant l_{1} \leqslant l_{2} \leqslant l_{1}+1$,

$$
\lim _{\beta \rightarrow \infty} \mathrm{P}_{\nu_{R_{l_{1}, l_{2}}}}\left(\tau_{\left.\mathcal{R}^{<\left(l_{1}, l_{2}\right)}<e^{\left[(2 U-\Delta)\left(l_{c}-2\right)+2 U-\delta\right] \beta}\right)=0 .} .\right.
$$

Indeed, it follows from Proposition 4.24 (i) that the saddle of exit from $\mathcal{R}^{\geqslant\left(l_{1}, l_{2}\right)}$ is $e^{\left[(2 U-\Delta)\left(l_{c}-2\right)+2 U\right] \beta}$.

(5) For $l_{c} \leqslant l_{1} \leqslant l_{2} \leqslant l_{1}+1$ we define a growing event $\mathbf{E}_{l_{1}, l_{2}}^{g}$ containing the set of trajectories starting from $\mathcal{R}_{l_{1}, l_{2}}$ and passing, at the successive times $\tau_{1}, \tau_{2}, \ldots$, through the following sequence $\psi^{g}=\psi_{1}^{g}, \psi_{2}^{g}, \ldots$ of pairs of integers:

$$
\begin{array}{ll}
\left(l_{1}, l_{2}\right)=(l, l): & \psi^{g}=(l, l),(l+1, l),(l+1, l+1), \ldots,\left(l_{0}, l_{0}\right), \\
\left(l_{1}, l_{2}\right)=(l, l+1): & \psi^{g}=(l, l+1),(l+1, l+1), \ldots,\left(l_{0}, l_{0}\right), \\
\left(l_{1}, l_{2}\right)=\left(l_{0}, l_{0}\right): & \psi^{g} \text { is the trivial path with no move. }
\end{array}
$$

More precisely, we denote by $\mathcal{R}_{\psi^{g}}$ the sequence of sets of configurations $\mathcal{R}_{l, l^{\prime}}$ with $\left(l, l^{\prime}\right)$ following the sequence $\psi^{g}$, and define

$$
\mathbf{E}_{l_{1}, l_{2}}^{g}=\left\{\phi: \phi_{\tau_{0}} \in \mathcal{R}_{l_{1} l_{2}}, \phi_{\tau_{i}} \in \mathcal{R}_{\psi_{i}^{g}} \forall i \in \mathbb{N}, \max _{i}\left(\tau_{i+1}-\tau_{i}\right)<e^{(2 \Delta-U+\delta) \beta}\right\} .
$$

(6) In the following we abbreviate

$$
\begin{aligned}
& \delta_{s}=\frac{1}{2}\left((2 \Delta-U)-\left[(2 U-\Delta)\left(l_{c}-3\right)+2 U\right]\right), \\
& \delta_{g}=\frac{1}{2}\left(\left[(2 U-\Delta)\left(l_{c}-2\right)+2 U\right]-(2 \Delta-U)\right) .
\end{aligned}
$$

(7) In the subcritical case $0 \leqslant l_{1} \leqslant l_{2} \leqslant l_{1}+1, l_{1} \leqslant l_{c}$ we have, for $\beta$ sufficiently large,

$$
\mathbb{P}_{\nu_{\mathcal{R}_{l_{1}, l_{2}}}}\left(\tau_{\square}<\tau_{\square}\right) \leqslant \mathbb{P}_{\nu_{\mathcal{R}_{l_{1}, l_{2}}}}\left(\tau_{\mathcal{R}^{>}>\left(l_{1}, l_{2}\right)}<\tau_{\square}\right) .
$$

On the other hand, by (8.37) we have

$$
\mathbb{P}_{\nu_{\mathcal{R}_{l_{1}, l_{2}}}}\left(\tau_{\mathcal{R}^{>}>\left(l_{1} l_{2}\right)}<\tau_{\square}\right) \leqslant \mathbb{P}_{\nu_{\mathcal{R}_{l_{1}, l_{2}}}}\left(e^{\left[2 \Delta-U-\left(\delta_{s} / 2\right)\right] \beta}<\tau_{\mathcal{R}}>\left(l_{1}, l_{2}\right)<\tau_{\square}\right)+o(1) .
$$

Moreover, 


$$
\begin{aligned}
& \mathrm{P}_{\nu_{R_{l_{1}, l_{2}}}}\left(e^{\left[2 \Delta-U\left(\delta_{s} / 2\right)\right] \beta}<\tau_{\mathcal{R}^{>}>\left(l_{1}, l_{2}\right)}<\tau_{\square}\right) \\
& \leqslant \mathrm{P}_{\nu_{R_{l_{1}, l_{2}}}}\left(e^{\left[2 \Delta-U-\left(\delta_{s} / 2\right)\right] \beta}\right. \\
& \left.<\tau_{\mathcal{R}^{>\left(l_{1}, l_{2}\right)}}, e^{\left[(2 U-\Delta)\left(l_{1}-2\right)+2 U+\left(\delta_{s} / 2\right)\right] \beta}<\tau_{\square}\right) .
\end{aligned}
$$

We know from Proposition 7.9 that if $0 \leqslant l_{1} \leqslant l_{2} \leqslant l_{1}+1, l_{1} \leqslant l_{c}$, then for all $\delta>0$ and $\beta$ sufficiently large

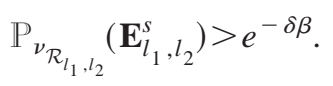

The first claim in Theorem 1.53(a) now follows from (8.42), (5.25), (5.27), (8.45), and Proposition 2.13 with

$$
\begin{gathered}
\nu=\nu_{\mathcal{R}_{l_{1} l_{2}},} \\
T=e^{\left[(2 U-\Delta)\left(l_{1}-2\right)+2 U+\left(\delta_{s} / 2\right)\right] \beta}, \quad T^{\prime}=T^{\prime \prime}=e^{\left[2 \Delta-U-\left(\delta_{s} / 2\right)\right] \beta}, \\
\mathcal{A}=\square, \quad \mathcal{B}=\mathcal{R}^{>\left(l_{1}, l_{2}\right)}, \quad p=e^{-\left(\delta_{s} / 4\right) \beta} .
\end{gathered}
$$

(8) In the supercritical case $l_{c} \leqslant l_{1} \leqslant l_{2} \leqslant l_{1}+1$, we proceed in a similar way. We have

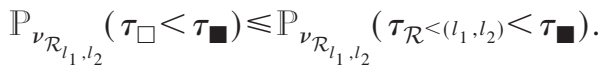

On the other hand, by (8.39) we have

$$
\mathrm{P}_{\nu_{\mathcal{R}_{l_{1}, l_{2}}}}\left(\tau_{\mathcal{R}^{<}<\left(l_{1}, l_{2}\right)<\tau_{\square}}\right) \leqslant \mathbb{P}_{\nu_{\mathcal{R}_{l_{1}, l_{2}}}}\left(e^{\left[(2 U-\Delta)\left(l_{c}-2\right)+2 U-\left(\delta_{g} / 2\right)\right] \beta}<\tau_{\mathcal{R}^{<\left(l_{1}, l_{2}\right)}<\tau_{\square}}\right)+o(1) .
$$

Moreover,

$$
\begin{aligned}
& \mathbb{P}_{\nu_{\mathcal{R}_{l_{1}, l_{2}}}}\left(e^{\left[(2 U-\Delta)\left(l_{c}-2\right)+2 U-\left(\delta_{g} / 2\right)\right] \beta}<\tau_{\mathcal{R}^{<}<\left(l_{1}, l_{2}\right)}<\tau_{\mathbf{\square}}\right)
\end{aligned}
$$

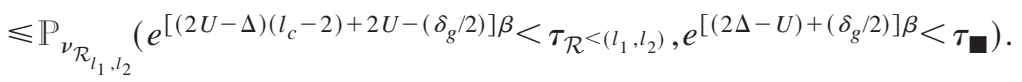

We know from Proposition 7.9 that if $l_{c} \leqslant l_{1} \leqslant l_{2} \leqslant l_{1}+1$, then for all $\delta>0$ and $\beta$ sufficiently large

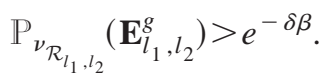

The second claim in Theorem 1.53(a) now follows from (5.29), (8.48), (8.50), and Proposition 2.13 with

$$
\begin{gathered}
\nu=\nu_{\mathcal{R}_{l_{1}, l_{2}},} \\
T=e^{\left[(2 \Delta-U)+\left(\delta_{g} / 2\right)\right] \beta}, \quad T^{\prime}=T^{\prime \prime}=e^{\left[(2 U-\Delta)\left(l_{c}-2\right)+2 U-\left(\delta_{g} / 2\right)\right] \beta}, \\
\mathcal{A}=\mathbf{\square}, \quad \mathcal{B}=\mathcal{R}^{<\left(l_{1}, l_{2}\right)}, \quad p=e^{-\left(\delta_{g} / 4\right) \beta} .
\end{gathered}
$$

\section{ACKNOWLEDGMENTS}

F.d.H. was a guest of the Mathematics Department, University of Rome Tor Vergata, in January 1998 and of the Instituto Nazionale di Fisica della Materia in July 1998. E.O. and E.S. 
were guests of EURANDOM, Technical University of Eindhoven, in April 1999. This work was partially supported by MURST-grant Confinanziamento 1998 Scienze Fisiche/21. E.O. and E.S. thank Oliver Penrose for stimulating discussions during the early stages of this work. The authors thank Marek Biskup for preparing the figures.

\section{APPENDIX: COMPARISON OF ENSEMBLES}

The grand-canonical Gibbs measure for our system on a torus $\Lambda=\Lambda_{\beta} \subset Z^{2}$ with activity $z$ is

$$
\mu_{\Lambda, z}\left(\eta_{\Lambda}\right)=\left[\prod_{x \in \Lambda} \frac{z^{\eta(x)}}{\eta(x) !}\right] \frac{\exp \left[-\beta H\left(\eta_{\Lambda}^{-}\right)\right]}{Z(\Lambda, z, \beta)} \quad\left(\eta_{\Lambda} \in \mathcal{X}_{\Lambda}\right),
$$

where $\mathcal{X}_{\Lambda}=\{0,1\}^{\bar{\Lambda}} \times \mathbb{N}^{\Lambda \backslash \bar{\Lambda}}, \eta_{\bar{\Lambda}}$ is the restriction of $\eta_{\Lambda}$ to $\bar{\Lambda}$,

$$
H\left(\eta_{\bar{\Lambda}}\right)=-U \sum_{(x, y) \in \bar{\Lambda}_{0}} \eta(x) \eta(y) \quad\left(\eta_{\bar{\Lambda}} \in \mathcal{X}_{\bar{\Lambda}}\right),
$$

$\mathcal{X}_{\bar{\Lambda}}^{-}=\{0,1\}^{\bar{\Lambda}}$, and

$$
Z(\Lambda, z, \beta)=e^{z|\Lambda \backslash \bar{\Lambda}|} \sum_{\eta_{\bar{\Lambda}} \in \mathcal{X}_{\bar{\Lambda}}^{-}} \exp \left[-\beta H\left(\eta_{\bar{\Lambda}}^{-}\right)\right] z^{\Sigma_{x \in \bar{\Lambda}} \eta(X)} .
$$

The canonical Gibbs measure with $n$ particles is

$$
\nu_{\Lambda, n}\left(\eta_{\Lambda}\right)=\left[\prod_{x \in \Lambda \backslash \bar{\Lambda}} \frac{1}{\eta(x) !}\right] \frac{\exp \left[-\beta H\left(\eta_{\bar{\Lambda}}\right)\right]}{Z(\Lambda, n, \beta)} \quad\left(\eta_{\Lambda} \in \mathcal{X}_{\Lambda, n}\right),
$$

where $\mathcal{X}_{\Lambda, n}=\left\{\eta_{\Lambda} \in \mathcal{X}_{\Lambda}: \Sigma_{x \in \Lambda} \eta(x)=n\right\}$ and

$$
Z(\Lambda, n, \beta)=\sum_{\eta_{\Lambda} \in \mathcal{X}_{\Lambda, n}}\left[\prod_{x \in \Lambda \backslash \bar{\Lambda}} \frac{1}{\eta(x) !}\right] \exp \left[-\beta H\left(\eta_{\bar{\Lambda}}^{-}\right)\right] .
$$

It is straightforward to verify that both these measures are reversible with respect to the Kawasaki dynamics with Hamiltonian $H$ in (1.47).

We want to compare the expected values with respect to the above two measures of a cylindrical function $f$ with support in $\bar{\Lambda}$. In what follows, we give an elementary estimate showing that the difference between the two expectations is inversely proportional to the total volume $|\Lambda|$. With the help of asymptotic expansions, like the ones used to get a local central limit theorem, it would be possible to get better estimates, even in more complicated situations (see $\mathrm{Yau}^{22}$ and Bertini, Cirillo, and Olivieri ${ }^{23}$ ). However, for our purpose it suffices to have a rough estimate.

Proposition A6: There exists $c=c(\bar{\Lambda})>0$ such that, for all $f: \mathcal{X}_{\bar{\Lambda}} \rightarrow \mathbb{R}$ with $\|f\|_{\infty} \leqslant \frac{1}{4}$, all $n$ $\in \mathbb{N}$ and all $\Lambda \supset \bar{\Lambda}$,

$$
\left|\nu_{\Lambda, n}(f)-\mu_{\Lambda, z}(f)\right| \leqslant \frac{c}{|\Lambda|} \quad \text { when } z=\frac{n}{|\Lambda|} .
$$

Proof: (1) Put $\nu=\nu_{\Lambda, n}$ and $\mu=\mu_{\Lambda, z}$. We have

$$
|\nu(f)-\mu(f)|=\frac{\mu^{g}\left(1_{N=n}\right)}{\mu\left(1_{N=n}\right)}-1,
$$


where $N=N(\eta)=\Sigma_{x \in \Lambda} \eta(x), g=f-\mu(f)$ and $\mu^{g}(h)=\mu((1+g) h)$. Note that $\mu^{g}$ is a probability measure: $\mu^{g}(\eta) \geqslant 0$ and $\mu^{g}(1)=1,\|g\|_{\infty} \leqslant 2\|f\|_{\infty} \leqslant \frac{1}{2}$ and $g$ has mean zero under $\mu$.

(2) Let $\bar{N}=\bar{N}(\eta)=\Sigma_{x \in \bar{\Lambda}} \eta(x)$ and $\hat{N}=\hat{N}(\eta)=\Sigma_{x \in \Lambda \backslash \bar{\Lambda}} \eta(x)$. From (A1) we see that the grand-canonical measure factorizes

$$
\begin{aligned}
& \mu\left(\eta_{\Lambda}\right)=\mu_{\bar{\Lambda}}\left(\eta_{\bar{\Lambda}}\right) \mu_{\Lambda \backslash \bar{\Lambda}}\left(\eta_{\Lambda \backslash \bar{\Lambda}}\right), \\
& \mu^{g}\left(\eta_{\Lambda}\right)=\mu_{\bar{\Lambda}}^{g}\left(\eta_{\bar{\Lambda}}\right) \mu_{\Lambda \backslash \bar{\Lambda}}\left(\eta_{\Lambda \backslash \bar{\Lambda}}\right),
\end{aligned}
$$

where

$$
\begin{gathered}
\mu_{\bar{\Lambda}}^{-}\left(\eta_{\bar{\Lambda}}^{-}\right)=\frac{\exp \left[-\beta H\left(\eta_{\bar{\Lambda}}^{-}\right)\right] z^{\bar{N}\left(\eta_{\Lambda}^{-}\right)}}{\sum_{\eta_{\bar{\Lambda}} \in \overline{\mathcal{X}}^{-}} \exp \left[-\beta H\left(\eta_{\bar{\Lambda}}^{-}\right)\right] z^{N\left(\eta_{\Lambda}^{-}\right)}}, \\
\mu_{\bar{\Lambda}}^{g}\left(\eta_{\bar{\Lambda}}\right)=\frac{\left(1+g\left(\eta_{\bar{\Lambda}}^{-}\right)\right) \exp \left[-\beta H\left(\eta_{\bar{\Lambda}}^{-}\right)\right] z^{\bar{N}\left(\eta_{\bar{\Lambda}}^{-}\right)}}{\sum_{\eta_{\bar{\Lambda}} \in \overline{\mathcal{X}}} \exp \left[-\beta H\left(\eta_{\bar{\Lambda}}^{-}\right)\right] z^{N\left(\eta_{\bar{\Lambda}}\right)}},
\end{gathered}
$$

and

$$
\mu_{\Lambda \backslash \bar{\Lambda}}\left(\eta_{\Lambda \backslash \bar{\Lambda}}\right)=e^{-z|\Lambda \backslash \bar{\Lambda}|} \prod_{z \in \Lambda \backslash \bar{\Lambda}} \frac{\lambda^{\eta(x)}}{\eta(x) !} .
$$

From (A9) we have

$$
\begin{gathered}
\mu\left(1_{N=n}\right)=\mu(N=n)=\sum_{\bar{n}=0}^{|\bar{\Lambda}|} \mu_{\bar{\Lambda}}(\bar{N}=\bar{n}) \mu_{\Lambda \backslash \bar{\Lambda}}(\hat{N}=n-\bar{n}), \\
\mu^{g}\left(1_{N=n}\right)=\mu^{g}(N=n)=\sum_{\bar{n}=0}^{|\bar{\Lambda}|} \mu_{\bar{\Lambda}}^{g}(\bar{N}=\bar{n}) \mu_{\Lambda \backslash \bar{\Lambda}}(\hat{N}=n-\bar{n}),
\end{gathered}
$$

(3) Set $k=|\Lambda \backslash \bar{\Lambda}|$ and $m=n-\bar{n}$. Then from (A11) we get

$$
\mu_{\Lambda \backslash \bar{\Lambda}}(\hat{N}=m)=\frac{(z k)^{m} e^{-z k}}{m !} .
$$

Thus

$$
\mu_{\Lambda \backslash \bar{\Lambda}}(\hat{N}=n-\bar{n})=\mu_{\Lambda \backslash \bar{\Lambda}}(\hat{N}=n-|\bar{\Lambda}|) \phi_{\bar{\Lambda}}(\bar{n})
$$

with

$$
\phi_{\bar{\Lambda}}(\bar{\eta})=\frac{(z k)^{|\bar{\Lambda}|-\bar{n}}}{(n-\bar{n})(n-\bar{n}-1) \times \cdots \times(n-|\bar{\Lambda}|+1)} .
$$

Substitution of (A14) into (A12) gives

$$
\frac{\mu^{g}(N=n)}{\mu(N=n)}=\frac{\sum_{\bar{n}=0}^{|\bar{\Lambda}|} \mu_{\bar{\Lambda}}^{g}(N=n) \phi_{\bar{\Lambda}}^{-}(\bar{n})}{\sum_{\bar{n}=0}^{|\bar{\Lambda}|} \mu_{\bar{\Lambda}}^{-}(N=n) \phi_{\bar{\Lambda}}(\bar{n})} .
$$

From (A15) it follows that there exists a $c=c(|\bar{\Lambda}|)>0$ such that for all $\Lambda \supset \bar{\Lambda}$ 


$$
\sup _{0 \leqslant \bar{n} \leqslant|\bar{\Lambda}|}\left|\phi_{\bar{\Lambda}}(\bar{n})-1\right| \leqslant \frac{c}{|\Lambda|} .
$$

(4) From (A8), (A16), and (A17) the result in (A7) follows. QED

Remark: It is clear from the above calculation that the assumption $\|f\|_{\infty} \leqslant \frac{1}{4}$ does not represent any loss of generality: in the generic case we get $4 c\|f\|_{\infty}$ instead of $c$ in the r.h.s. of (A7). Moreover, the same estimate holds when the support $\bar{\Lambda}$ of $f$ is replaced by any $\Lambda^{\prime} \supset \bar{\Lambda}$ : we get a different constant $c=c\left(\Lambda^{\prime}\right)$. It is easy to check that $c\left(\Lambda^{\prime}\right) \leqslant \kappa\left|\Lambda^{\prime}\right|$ for some $\kappa>0$.

${ }^{1}$ D. Ruelle, Statistical Mechanics (Benjamin, New York, 1969).

${ }^{2}$ Ya. G. Sinai, Theory of Phase Transitions: Rigorous Results (Akademiai Kiado, Budapest, 1982).

${ }^{3} \mathrm{H}$. Tomita and S. Miyashita, "Statistical properties of the relaxation process of metastable states in the kinetic Ising model," Phys. Rev. B 46, 8886-8893 (1992).

${ }^{4}$ E. J. Neves and R. H. Schonmann, "Critical droplets and metastability for a Glauber dynamics at very low temperatures,' Commun. Math. Phys. 137, 209-230 (1999).

${ }^{5}$ E. J. Neves and R. H. Schonmann, "Behavior of droplets for a class of Glauber dynamics at very low temperatures," Prob. Theory Relat. Fields 91, 331-354 (1992).

${ }^{6}$ R. H. Schonmann, “The pattern of escape from metastability of a stochastic Ising model," Commun. Math. Phys. 147, 231-240 (1992).

${ }^{7}$ R. H. Schonmann, "Slow droplet driven relaxation of stochastic Ising models in the vicinity of the phase coexistence region," Commun. Math. Phys. 161, 1-49 (1994).

${ }^{8}$ R. H. Schonmann, "Theorems and conjectures on the droplet-driven relaxation of stochastic Ising models," in Probability and Phase Transition, edited by G. Grimmett, NATO ASI Series C, Vol. 420 (Kluwer, Dordrecht, 1994), pp. 265-301.

${ }^{9}$ M. I. Freidlin and A. D. Wentzell, Random Perturbations of Dynamical Systems (Springer, New York, 1984).

${ }^{10}$ E. Olivieri and E. Scoppola, "Markov chains with exponentially small transition probabilities: First exit problem from a general domain. I. The reversible case," J. Stat. Phys. 79, 613-647 (1995).

${ }^{11}$ E. Olivieri and E. Scoppola, "Markov chains with exponentially small transition probabilities: First exist problem from a general domain. II. The general case," J. Stat. Phys. 84, 987-1041 (1996).

${ }^{12}$ O. Catoni and R. Cerf, "The exit path of a Markov chain with rare transitions," ESAIM Probab. Stat. 1995/1997, pp. 95-144.

${ }^{13}$ E. Olivieri and E. Scoppola, "Metastability and typical exit paths in stochastic dynamics," in Proceedings of the European Congress of Mathematics, Budapest, 22-26 July, 1996, edited by A. Balog, G. O. H. Katona, A. Recski, D. Szász, Progr. Math. 169 (Birkhäuser, Boston, 1998), pp. 124-150.

${ }^{14}$ A. Trouvé, "Cycle decompositions and simulated annealing,"' SIAM J. Control Optim. 3, 966-986 (1996).

${ }^{15}$ J. Lebowitz and O. Penrose, "Rigorous treatment of metastable states in van der Waals theory," J. Stat. Phys. 3, 211-236 (1971).

${ }^{16}$ D. Capocaccia, M. Cassandro, and E. Olivieri, “A study of metastability in the Ising model," Commun. Math. Phys. 39, 185-205 (1974).

${ }^{17}$ P. Deghampour and R. H. Schonmann, “Metropolis dynamics relaxation via nucleation and growth," Commun. Math. Phys. 188, 89-119 (1997).

${ }^{18}$ F. Spitzer, Principles of Random Walk, 2nd ed. (Springer, New York, 1976).

${ }^{19}$ G. Gallavotti, "Instabilities and phase transitions in the Ising model. A review," Riv. Nuovo Cimento 2, 133-169 (1972).

${ }^{20}$ E. Scoppola, “Renormalization group for Markov chains and application to metastability," J. Stat. Phys. 73, 83-121 (1993).

${ }^{21}$ C. Peixoto, “An exclusion process with Metropolis rate I," Markov Processes Relat. Fields (to appear).

${ }^{22}$ H. T. Yau, "Logarithmic Sobolev inequalities for lattice gases with mixing conditions," Commun. Math. Phys. 181, 367-408 (1996).

${ }^{23}$ L. Bertini, E. Cirillo, and E. Olivieri, "Renormalization group maps: Gibbsianness and convergence of renormalized interactions under strong mixing conditions," J. Stat. Phys. (to appear). 